\title{
Elastic Response of Acoustic Coating on Fluid-Loaded Rib-Stiffened Cylindrical Shells
}

\author{
Christopher Gilles Doherty
}

Thesis submitted to the faculty of the Virginia Polytechnic Institute and State University in partial fulfillment of the requirements for the degree of

Master of Science

In

Mechanical Engineering

\author{
Steve Southward, Chair \\ Mehdi Ahmadian \\ Andrew Kurdila
}

April $28^{\text {th }}, 2017$

Blacksburg, VA USA

Keywords: Reinforced cylinder, acoustic coating, elasticity, orthogonalization 


\title{
Elastic Response of Acoustic Coating on Fluid-Loaded Rib-Stiffened Cylindrical Shells
}

\author{
Christopher Gilles Doherty
}

\section{Abstract}

Reinforced cylindrical shells are used in numerous industries; common examples include undersea vehicles and industrial piping. Current models typically incorporate approximate theories to determine shell behavior, which have limitations in terms of both thickness and frequency. In addition, many applications feature coatings on the shell surface that normally have thicknesses which must also be considered. To increase the fidelity of such systems, this work develops an analytical model of an elastic cylindrical shell featuring periodically spaced ring stiffeners with an acoustic coating applied to the outer surface. There is an external fluid environment. Beginning with the equations of elasticity for a solid, spatial-domain displacement field solutions are produced incorporating unknown wave propagation coefficients. These fields are used to determine stresses at the boundaries of the shell and coating, which are then coupled with stresses from the stiffeners and fluid. The stress boundary conditions contain double-index infinite summations, which are decoupled, truncated, and recombined into a global matrix equation. The solution to this global equation results in the displacement responses of the system as well as the scattered pressure field. Two distinct loadings are considered: a ring loading and an incident acoustic wave. Thin-shell reference models are used for validation, and the acoustic response of the system is examined. It is shown that the reinforcing ribs and acoustic coating have a considerable effect on system behavior. 


\title{
Elastic Response of Acoustic Coating on Fluid-Loaded Rib-Stiffened Cylindrical Shells
}

\author{
Christopher Gilles Doherty
}

\section{General Audience Abstract}

Reinforced cylindrical shells are used in numerous industries; common examples include undersea vehicles and industrial piping. Current models typically incorporate approximate theories to determine shell behavior, which have limitations in terms of both thickness and frequency. In addition, many applications feature coatings on the shell surface that normally have thicknesses which must also be considered. To increase the fidelity of such systems, this work develops an analytical model of an elastic cylindrical shell featuring periodically spaced ring stiffeners with an acoustic coating applied to the outer surface. There is an external fluid environment. Beginning with elastic equations of motion for a solid, the displacements of the system can be found. These displacements are used to determine stresses at the boundaries of the shell and coating, which are then coupled with stresses from the stiffeners and fluid. Techniques are used to transform the stress boundary conditions into a large matrix equation, and the solution to this global equation results in the displacement responses of the system as well as the scattered pressure field. Two distinct loadings are considered: a ring loading and an incident acoustic wave. Thin-shell reference models are used for validation, and the acoustic response of the system is examined. It is shown that the reinforcing ribs and acoustic coating have a considerable effect on system behavior. 


\section{Acknowledgements}

I would like to acknowledge my advisor Dr. Steve Southward for leading this project and for his guidance throughout the process from start to finish. Additionally, I would like to thank Dr. Andrew Hull of the United States Naval Undersea Warfare Center for his contributions regarding the techniques used in this work, and for his assistance finding solutions for the problems we inevitably encountered. Finally, I must recognize my committee members Dr. Mehdi Ahmadian and Dr. Andrew Kurdila for their advice and support. This work was sponsored by the Naval Undersea Research Program at the Office of Naval Research.

This project is dedicated to Rebecca, Lillers and the Fuzz. 


\section{Contents}

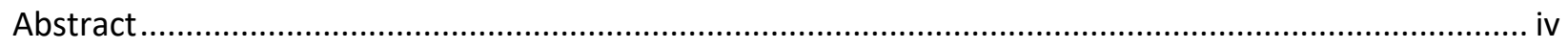

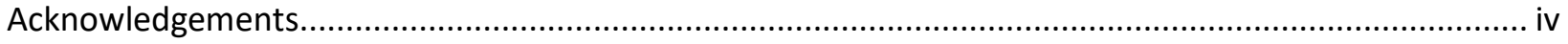

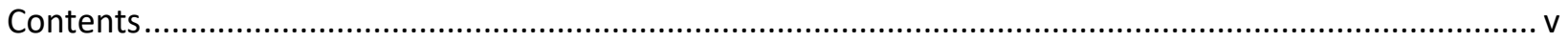

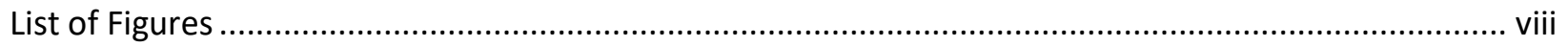

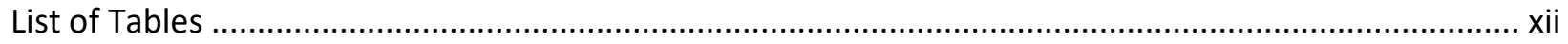

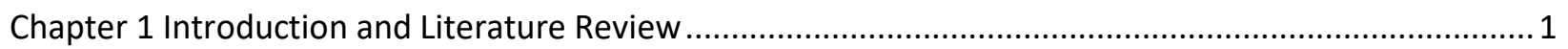

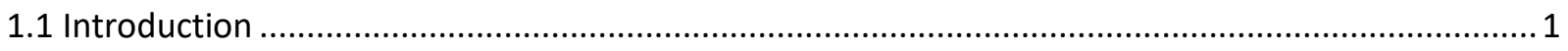

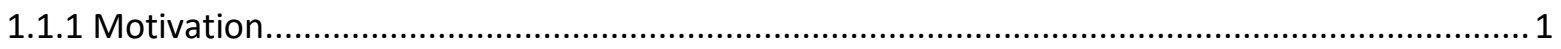

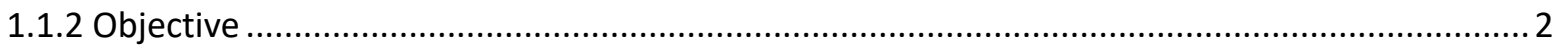

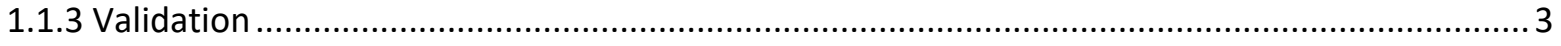

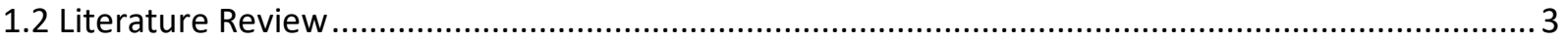

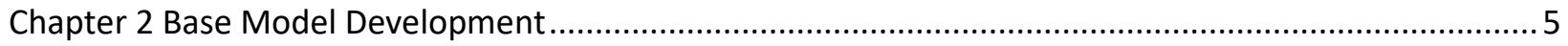

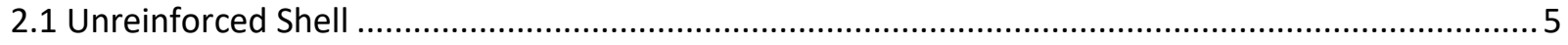

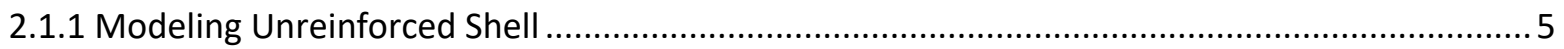

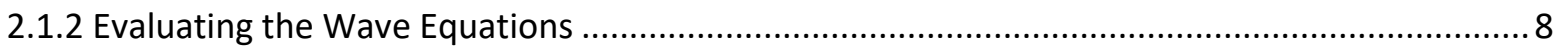

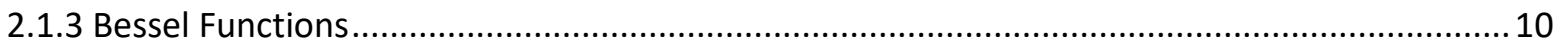

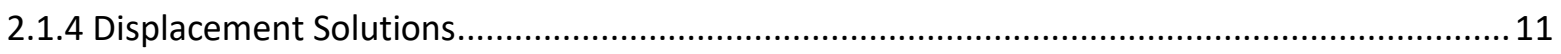

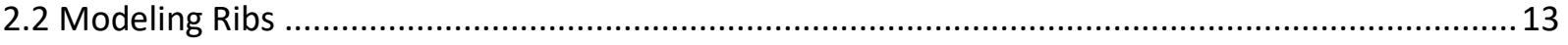

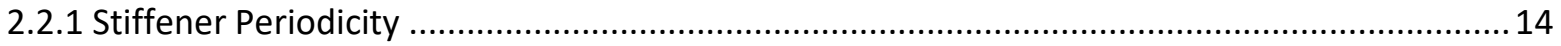

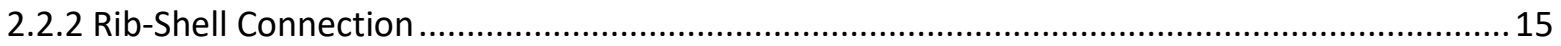

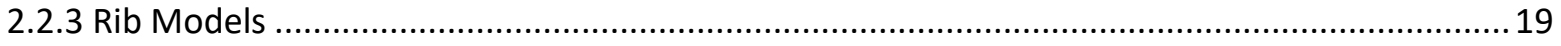

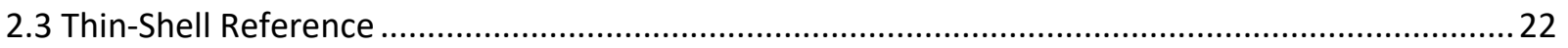

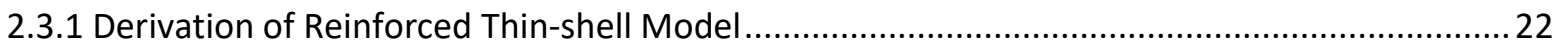

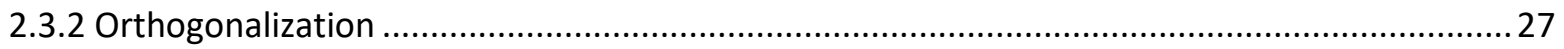

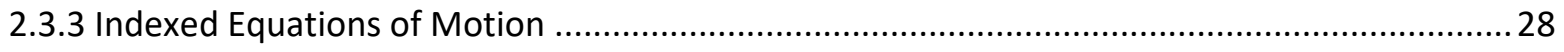

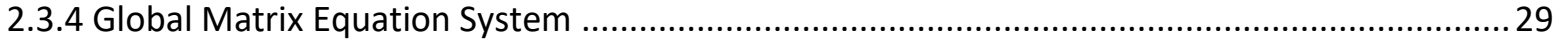

2.3.5 Modifications for Delta Rib Connectors ................................................................................ 30

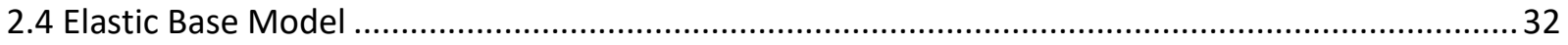

2.4.1 Derivation of Reinforced Thick-Shell Model ......................................................................... 32

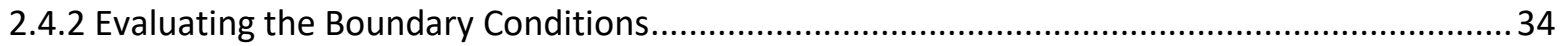




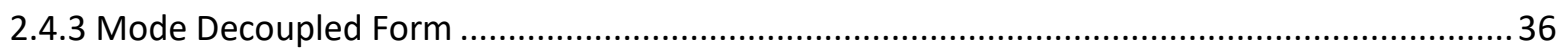

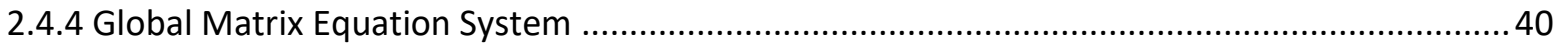

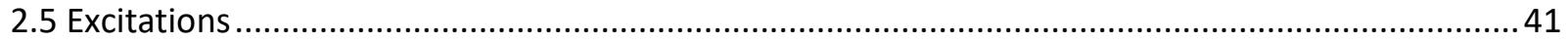

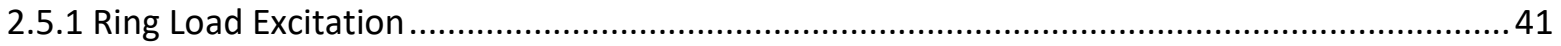

2.5.2 Incident Plane Wave (Acoustic Wave) …............................................................................. 42

2.5.3 Modifications to System Equations for Plane Wave Input ................................................. 45

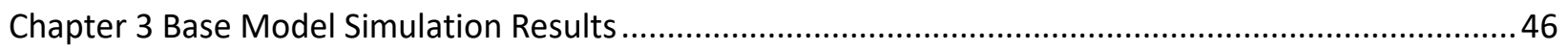

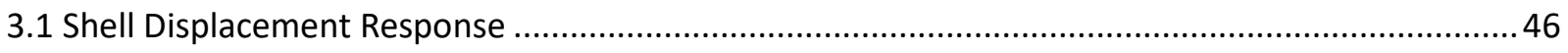

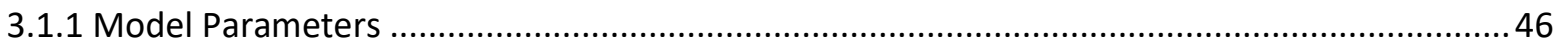

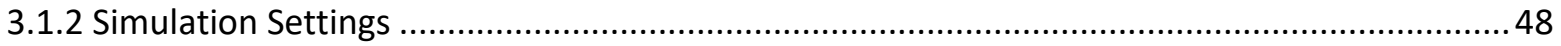

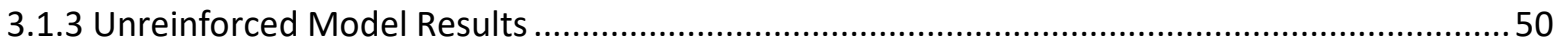

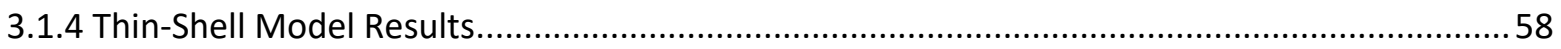

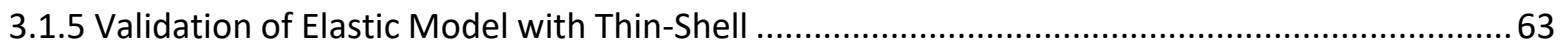

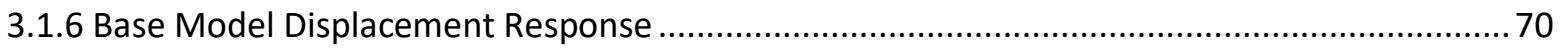

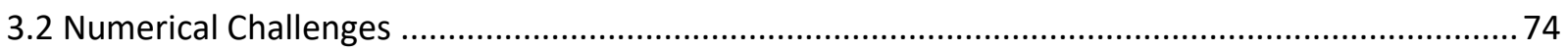

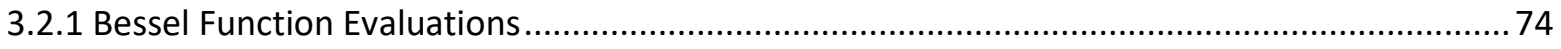

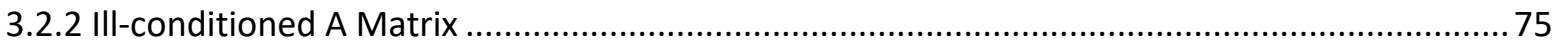

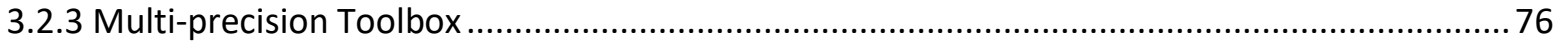

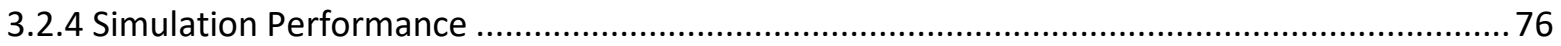

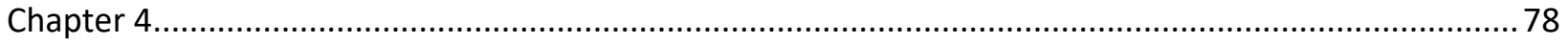

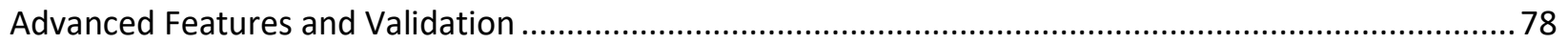

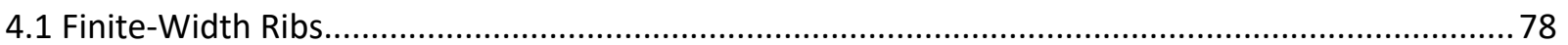

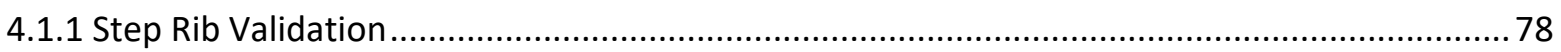

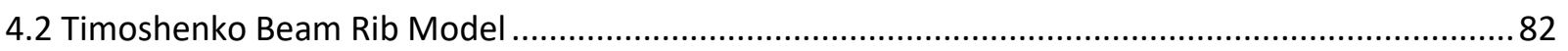

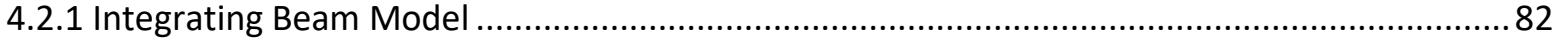

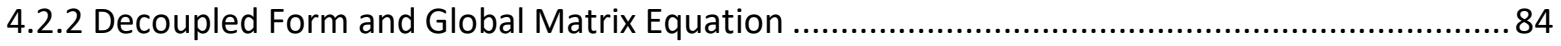

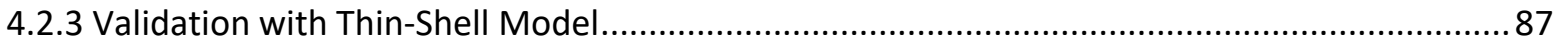

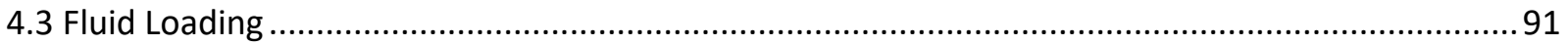

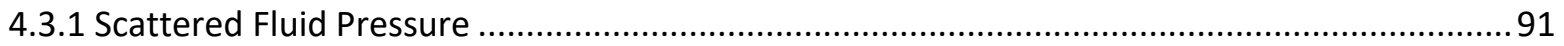

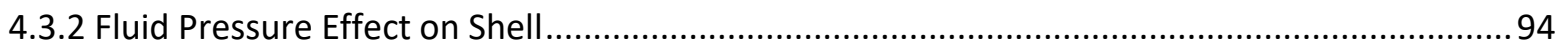

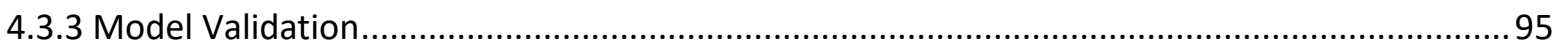

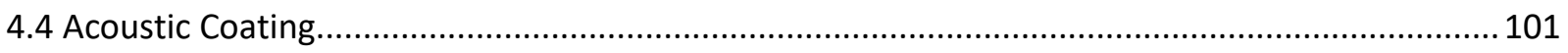

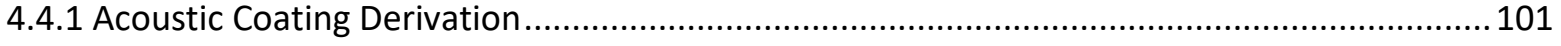




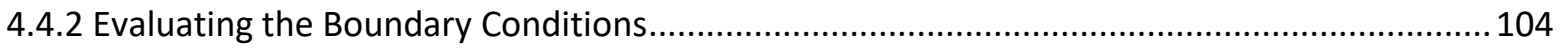

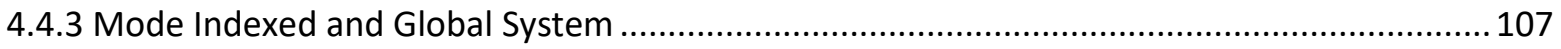

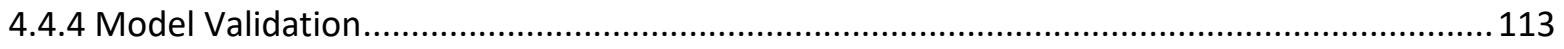

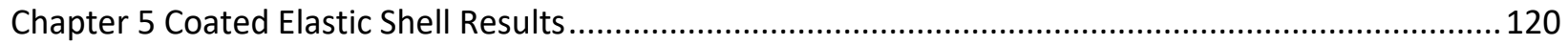

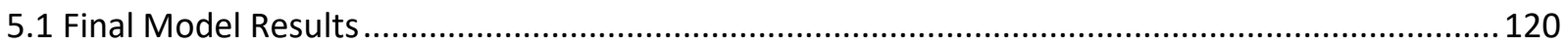

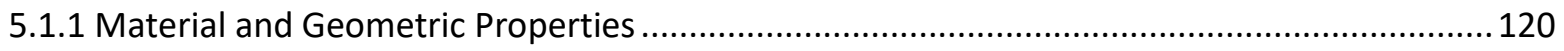

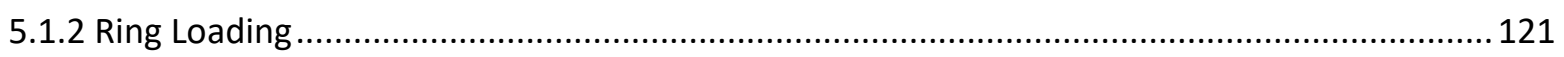

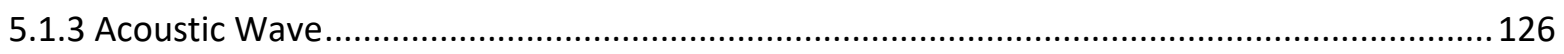

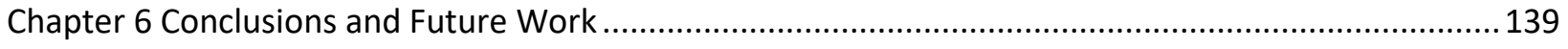

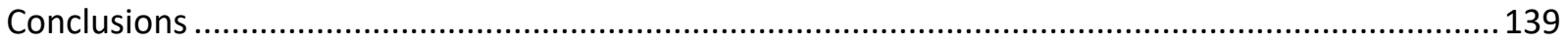

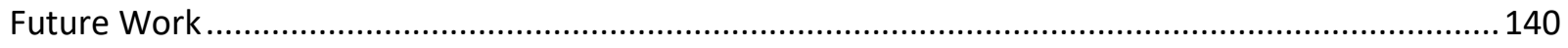

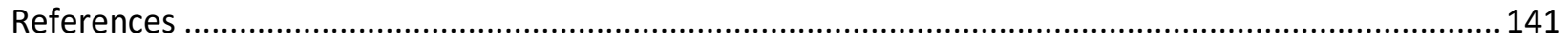

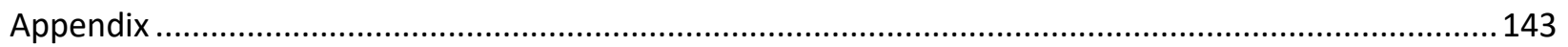

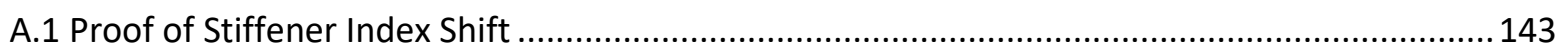

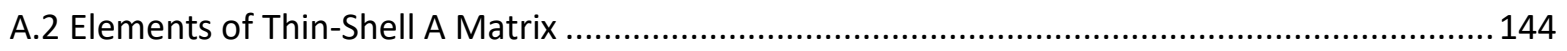

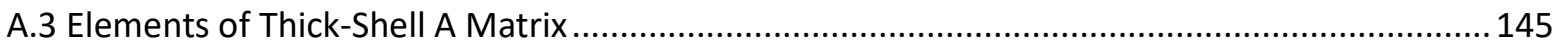

A.4 Timoshenko Curved Beam Equations ................................................................................ 146

A.5 Solution of the 3D Wave Equation (for Scalars) ................................................................. 150 


\section{List of Figures}

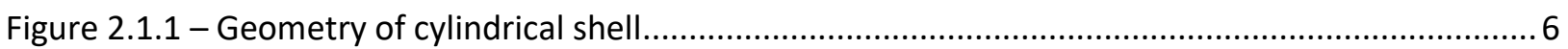

Figure 2.1.2 - Bessel Functions of first kind (left) and second kind (right) for order $n=0,1,2 \ldots \ldots \ldots \ldots . . .10$

Figure 2.1.3 - Modified Bessel Functions of first kind (left) and second kind (right) for order $n=0,1,211$

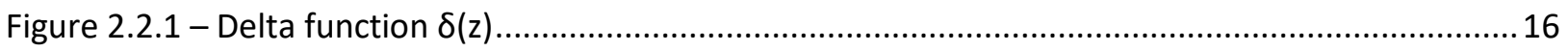

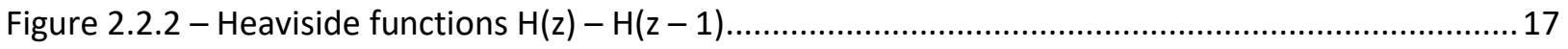

Figure 2.2.3 - Rib cross-sectional dimensions shown on the left in the rz-plane, and radial dimensions

shown on the right in the $r \theta$-plane. Rib shown in red, shell in gray ...........................................22

Figure 2.3.1 - Diagram of the thin-shell system geometry including stiffening ribs .............................23

Figure 2.3.2 - Diagram of the thin-shell system geometry with Delta stiffening ribs ..............................31

Figure 2.4.1 - Diagram of the thick-shell system geometry including the Delta ribs ...............................32

Figure 2.5.2 - Acoustic wave exciting outer cylinder surface, with angle of incidence, $\phi_{i} \ldots \ldots \ldots \ldots \ldots \ldots \ldots . . . . . . . . . .43$

Figure 2.5.2 - Plane Wave excitation applied to the left side of unreinforced cylindrical shell...............44

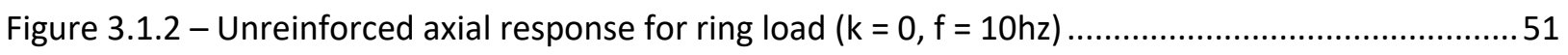

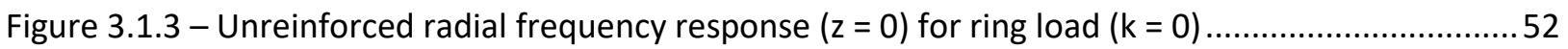

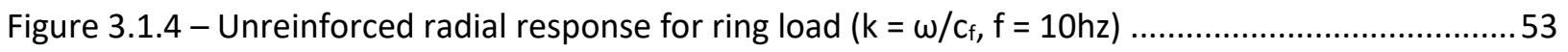

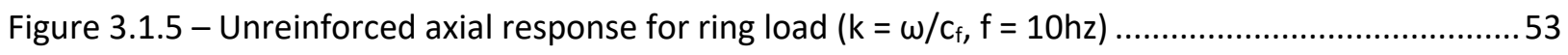

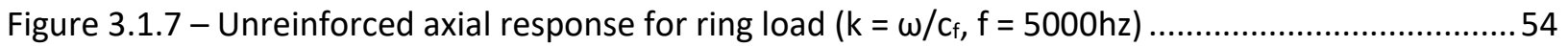

Figure 3.1.8 - Unreinforced radial/axial frequency response $(z=0)$ for ring load $\left(k=\omega / c_{f}\right) \ldots \ldots \ldots \ldots \ldots \ldots . . . . . . .55$

Figure 3.1.9 - Unreinforced cylinder radial (top), tangential (mid), and axial (bottom) displacement

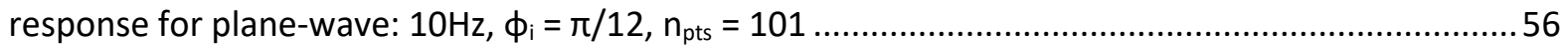

Figure 3.1.10 - Unreinforced cylinder radial (top), tangential (mid), and axial (bottom) displacement

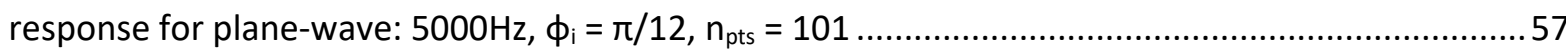

Figure 3.1.11 - Unreinforced radial/axial/tangential frequency response $(z=0, \theta=36 \mathrm{deg})$ for plane-

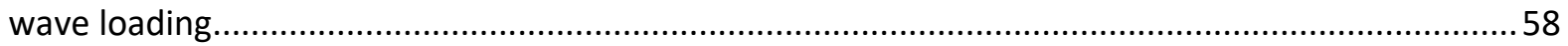

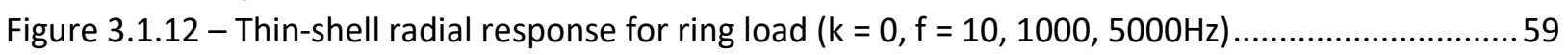

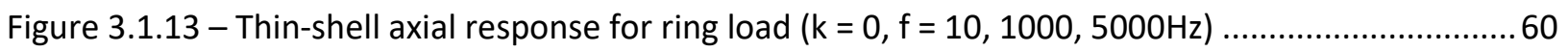

Figure 3.1.14 - Thin-shell radial response for $\operatorname{ring}$ load $\left(k=\omega / c_{f}, f=10,1000,5000 \mathrm{~Hz}\right) \ldots \ldots \ldots \ldots \ldots \ldots \ldots . . .61$

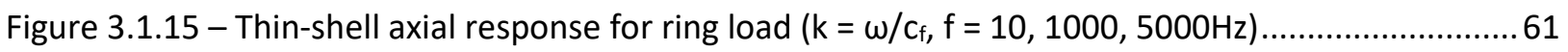

Figure 3.1.16 - Thin-shell cylinder radial (top), tangential (mid), and axial (bottom) displacement

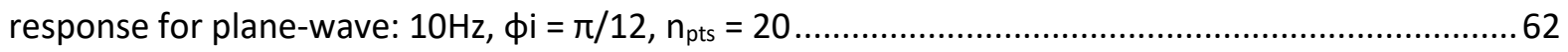

Figure 3.1.17 - Thin-shell cylinder radial (top), tangential (mid), and axial (bottom) displacement

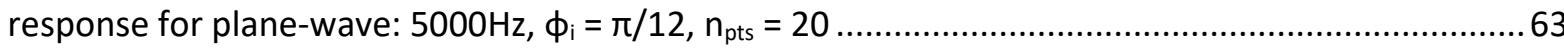

Figure 3.1.18 - Unreinforced models compared for $\mathrm{h}=0.001 \mathrm{~m}$ (left) and $\mathrm{h}=0.05 \mathrm{~m}$ (right) ...............64

Figure 3.1.19 - Comparison of thin and thick-shell $\left(m_{\text {pts }}=31\right)$ radial displacement excited by 10,50 and

$90 \mathrm{~Hz}$ Ring Loading $(\mathrm{k}=0)$

Figure 3.1.20 - Comparison of thin and thick-shell $\left(m_{p t s}=31\right)$ axial displacement excited by 10,50 and

$90 \mathrm{~Hz}$ Ring Loading $(\mathrm{k}=0)$ 66 
Figure 3.1.21 - Magnitude of displacement modes for each axial index, $m$.

Figure 3.1.22 - Thin and Thick-shell radial (top), tangential (mid), and axial (bottom) Normalized Error in

$\mathrm{dB}$ for plane-wave: $50 \mathrm{~Hz}, \phi \mathrm{i}=\pi / 12$, $\mathrm{mpts}=31$, npts $=7$. .68

Figure 3.1.23 - Magnitude of displacement modes for each axial index, $m$..........................................69

Figure 3.1.24 - Magnitude of displacement modes for each circumferential index, $n$.........................69

Figure 3.1.25 - Normalized magnitude of displacement modes for each circumferential index, $n$ and axial index, $m$.

Figure 3.1.26 - Base model radial displacement response excited by 50, 1000 and $5000 \mathrm{~Hz}$ Ring Loading $(\mathrm{k}=0)$.

Figure 3.1.27 - Base model axial displacement response excited by 50, 1000 and $5000 \mathrm{~Hz}$ Ring Loading (k $=0)$...

Figure 3.1.28 - Elastic base model radial (top), tangential (mid), and axial (bottom) displacement response for acoustic-wave: $50 \mathrm{~Hz}, \phi_{\mathrm{i}}=15 \mathrm{deg}$ of broadside, $\mathrm{m}_{\mathrm{pts}}=31, \mathrm{n}_{\mathrm{pts}}=7$

Figure 3.1.29 - Elastic base model radial (top), tangential (mid), and axial (bottom) displacement response for acoustic-wave: $1000 \mathrm{~Hz}, \phi_{\mathrm{i}}=15 \mathrm{deg}, \mathrm{m}_{\mathrm{pts}}=31, \mathrm{n}_{\mathrm{pts}}=7$.

Figure 3.1.30 - Elastic base model radial (top), tangential (mid), and axial (bottom) displacement response for acoustic-wave: $5000 \mathrm{~Hz}, \phi_{\mathrm{i}}=15 \mathrm{deg}, \mathrm{m}_{\mathrm{pts}}=31, \mathrm{n}_{\mathrm{pts}}=7 .$.

Figure 4.1.1 - Comparison of reference $\left(m_{\text {pts }}=31\right)$ and elastic $\left(m_{p t s}=31\right)$ radial displacement magnitude for ring load $(\mathrm{k}=0)$

Figure 4.1.2 - Comparison of reference $\left(m_{p t s}=31\right)$ and elastic $\left(m_{p t s}=31\right)$ axial displacement magnitude for ring load $(k=0)$

Figure 4.1.3 - Thin and Thick-shell radial (top), tangential (mid), and axial (bottom) Normalized Error in $\mathrm{dB}$ for plane-wave: $50 \mathrm{~Hz}, \phi i=\pi / 12$, mpts $=31$, npts $=7$. Rib is located between the left edge $(z=0)$ and the red dashed line.

Figure 4.2.1 - Comparison of beam-rib reference and elastic radial displacement magnitude for ring load $(\mathrm{k}=0), \mathrm{m}_{\mathrm{pts}}=31$

Figure 4.2.2 - Comparison of beam-rib reference and elastic axial displacement magnitude for ring load $(\mathrm{k}=0), \mathrm{m}_{\mathrm{pts}}=31$

Figure 4.2.3 - Thin and Thick-shell radial (top), tangential (mid), and axial (bottom) Normalized Error in $\mathrm{dB}$ for plane-wave: $50 \mathrm{~Hz}, \phi i=\pi / 12$, $\mathrm{mpts}=31$, npts $=7$. Rib is located between the left edge $(z=0)$ and the red dashed line.

Figure 4.2.4 - Acoustic Wave radial response of reference and elastic model at $\theta=90 \mathrm{deg}$..................91

Figure 4.3.1 - Comparison of fluid reference and elastic radial displacement magnitude for ring load $(\mathrm{k}=$ $0), \mathrm{m}_{\mathrm{pts}}=31$.

Figure 4.3.2 - Comparison of fluid reference and elastic axial displacement magnitude for ring load $(\mathrm{k}=$ $0), \mathrm{m}_{\mathrm{pts}}=31$.

Figure 4.3.3 - Comparison of fluid reference and elastic radiated (top) and reflected (bottom) pressure

magnitude for ring load $(\mathrm{k}=0), \mathrm{m}_{\mathrm{pts}}=31$.

Figure 4.3.3 - Thin and Thick-shell radial (top), tangential (mid), and axial (bottom) Normalized Error in $\mathrm{dB}$ for plane-wave: $50 \mathrm{~Hz}, \phi_{\mathrm{i}}=\pi / 12, \mathrm{mpts}=31$, npts $=7$. Rib is located between the left edge $(z=0)$ and the red dashed line.

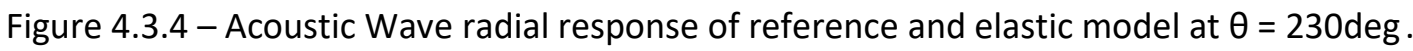
100 
Figure 4.3.5 - Thin and Thick-shell radiated pressure (top) and reflected pressure (bottom) Normalized Error in dB for plane-wave: $50 \mathrm{~Hz}, \phi_{\mathrm{i}}=\pi / 12$, $\mathrm{mpts}=31$, npts $=7$. Rib is located between the left edge $(\mathrm{z}=0)$ and the red dashed line. 101

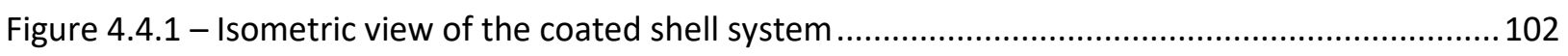

Figure 4.4.2 - Cross-section view of the coated shell system ........................................................ 102

Figure 4.4.3 - Comparison of radial displacement of reference and coated-elastic system excited by 10 ,

50 and $90 \mathrm{~Hz}$ Ring Loading $(\mathrm{k}=0), \mathrm{m}_{\mathrm{pts}}=31$

Figure 4.4.4 - Comparison of axial displacement of reference and coated-elastic system excited by 10, 50 and $90 \mathrm{~Hz}$ Ring Loading $(\mathrm{k}=0), \mathrm{m}_{\mathrm{pts}}=31$

Figure 4.4.5 - Thin and Thick-shell radial (top), tangential (mid), and axial (bottom) Normalized Error in $\mathrm{dB}$ for plane-wave: $50 \mathrm{~Hz}, \phi_{\mathrm{i}}=\pi / 12$, mpts $=31$, npts $=7$. Rib is located between the left edge $(z=0)$ and the red dashed line.

Figure 4.4.6 - Normalized magnitude of displacement modes for each circumferential index, $n$ and axial index, $m$

Figure 4.4.7 - Thin and Thick-shell radiated pressure (top) and reflected pressure (bottom) Normalized Error in dB for plane-wave: $50 \mathrm{~Hz}, \phi_{i}=\pi / 12$, $\mathrm{mpts}=31$, npts $=7$. Rib is located between the left edge $(z=0)$ and the red dashed line. 119

Figure 5.1.1 - Radial surface displacement response of coated elastic model excited by ring loading $(\mathrm{k}=$

0) at $50,1000,5000 \mathrm{~Hz}$

Figure 5.1.2 - Axial surface displacement response of coated elastic model excited by ring loading $(\mathrm{k}=0)$ at $50,1000,5000 \mathrm{~Hz}$ 122

Figure 5.1.3 - Radial displacement field of coated system for cross-section through the cylinder thickness at angle $\theta=0$, for ring loading $(k=0, f=50 \mathrm{~Hz})$; red lines are the rib.

Figure 5.1.4 - Axial displacement field of coated system for cross-section through the cylinder thickness at angle $\theta=0$, for ring loading $(k=0, f=50 \mathrm{~Hz})$.

Figure 5.1.5 - Radial displacement field of coated system for cross-section through the cylinder thickness at angle $\theta=0$, for ring loading $(k=0, f=5000 \mathrm{~Hz})$ 125

Figure 5.1.6 - Axial displacement field of coated system for cross-section through the cylinder thickness at angle $\theta=0$, for ring loading $(k=0, f=5000 \mathrm{~Hz})$...

Figure 5.1.7 - Coating surface radial (top), tangential (mid), and axial (bottom) displacement response for acoustic-wave: $50 \mathrm{~Hz}, \phi \mathrm{i}=15 \mathrm{deg}$, mpts $=31$, npts $=7$

Figure 5.1.8 - Coating surface radial (top), tangential (mid), and axial (bottom) displacement response for acoustic-wave: $1000 \mathrm{~Hz}, \phi i=15 \mathrm{deg}$, $\mathrm{mpts}=31$, npts $=7$

Figure 5.1.9 - Coating surface radial (top), tangential (mid), and axial (bottom) displacement response for acoustic-wave: $5000 \mathrm{~Hz}$, $\phi i=15 \mathrm{deg}$, mpts $=31$, npts $=7$

Figure 5.1.10 - Radial displacement field of coated system for cross-section of cylinder thickness at angle $\theta=10 \mathrm{deg}$, for acoustic wave loading $\left(\phi_{i}=15 \mathrm{deg}, f=5000 \mathrm{~Hz}\right)$; red lines represent the rib ....130

Figure 5.1.11 - Tangential displacement field of coated system for cross-section of cylinder thickness at angle $\theta=10 \mathrm{deg}$, for acoustic wave loading $\left(\phi_{i}=15 \mathrm{deg}, f=5000 \mathrm{~Hz}\right)$; red lines represent the rib ....131

Figure 5.1.12 - Axial displacement field of coated system for cross-section of cylinder thickness at angle $\theta=10 \mathrm{deg}$, for acoustic wave loading $\left(\phi_{i}=15 \mathrm{deg}, f=5000 \mathrm{~Hz}\right)$. Dashed black line represents a reference $15 \mathrm{deg}$ line; red lines represent the rib 132

Figure 5.1.10 - Radiated (top), and reflected (bottom) pressure response for acoustic-wave: $50 \mathrm{~Hz}, \phi_{\mathrm{i}}=$ $15 \mathrm{deg}, \mathrm{mpts}=31, \mathrm{npts}=7$ .133 
Figure 5.1.11 - Radiated (top), and reflected (bottom) pressure response for acoustic-wave: $1000 \mathrm{~Hz}, \phi_{\mathrm{i}}$

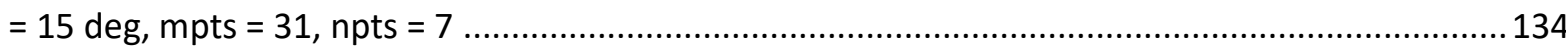

Figure 5.1.12 - Radiated (top), and reflected (bottom) pressure response for acoustic-wave: $5000 \mathrm{~Hz}, \phi_{\mathrm{i}}$ $=15 \mathrm{deg}, \mathrm{mpts}=31, \mathrm{npts}=7$

Figure 5.1.13 - Radiated pressure response versus angular position for acoustic-wave: $\phi_{\mathrm{i}}=15 \mathrm{deg}$, mpts $=31, \mathrm{npts}=7$ 136

Figure 5.1.14 - Reflected pressure response versus angular position for acoustic-wave: $\phi_{i}=15$ deg, mpts $=31$, npts $=7$ 136

Figure 5.1.15 - Comparison of radiated pressure response between Model 4 and 5 for acoustic-wave: $\phi_{i}$ $=15 \mathrm{deg}, \mathrm{mpts}=31, \mathrm{npts}=7$

Figure 5.1.16 - Comparison of reflected pressure response between Model 4 and 5 for acoustic-wave:

$\phi_{\mathrm{i}}=15 \mathrm{deg}, \mathrm{mpts}=31, \mathrm{npts}=7$ 138

Figure A.3.1 - FBD of infinitesimal section of circular beam .............................................................147

Figure A.3.2 - Curved beam element undergoing deformation and rotation. 148 


\section{List of Tables}

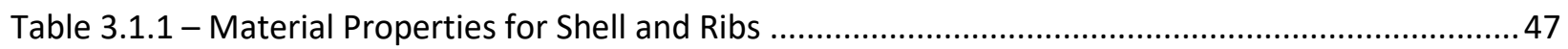

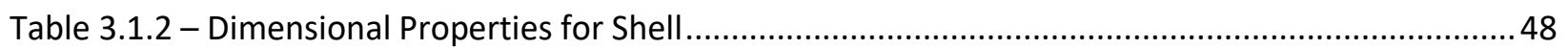

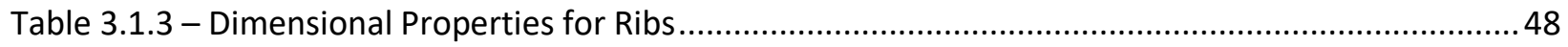

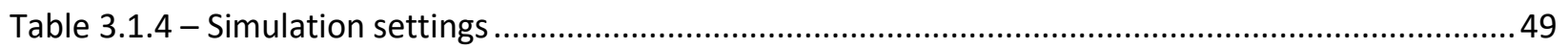

Table 3.1.5 - Unreinforced base model (elastic) settings...................................................................50

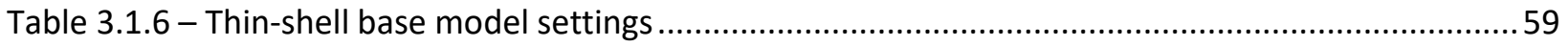

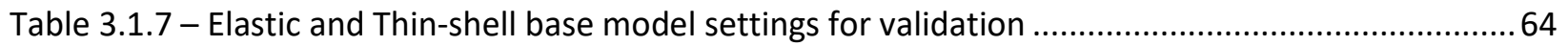

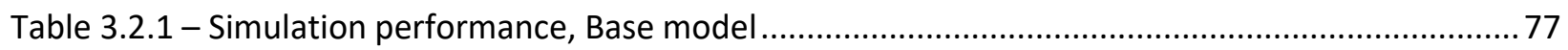

Table 3.2.2 - Simulation performance, Coated model ........................................................................... 77

Table 4.4.1 - Material and Geometry Properties for Shell and Coating ..............................................114

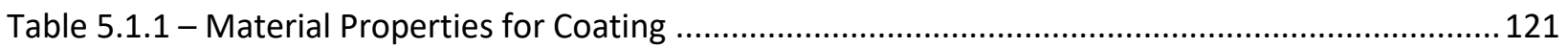

Table 5.1.2 - Dimensional Properties for Coating ................................................................................ 121 


\section{Chapter 1 Introduction and Literature Review}

\subsection{Introduction}

\subsubsection{Motivation}

Cylindrical shells can be found in a variety of applications, from industrial piping and pressure vessels to undersea vehicles. Often such systems experience large pressure differentials, either due to internal pressure or external loading from a fluid environment, which creates stresses in the shell and can lead to failure. To combat this issue, reinforcement is added to the shell to provide additional stiffness without significantly increasing the mass, offering an effective solution. Such reinforcement could be integrated into the shell itself (i.e. directional composites or rebar in concrete) or attached to the shell in some manner (i.e. ring stiffeners or longitudinal ribs). Unfortunately, the structural response of the system usually changes when fortification is implemented, and the interaction of the shell and the reinforcement must be considered.

Acoustic coatings are generally used for undersea applications to damp radiated vibrations, reduce drag, enclose sonar sensors, and protect the exterior of the vehicle. In reality, the coatings can have a significant effect on acoustic characteristics of the object. These polymer coatings can be very thick, often moreso than the body upon which they are applied, and often have more internal damping. While there are well known approximate theories present to describe such a combination, they are invalidated by both the thicknesses and the excitation frequencies.

This project combines the difficulty of modeling the elastic shell with periodic stiffeners and the effect of the acoustic coating on the shell. Both challenges must be met in order to create 
an accurate representation of a coated and reinforced elastic shell, which can be excited at high frequency. In the future, this work will lead to new models with finite length shells and variously shaped end-caps, but these remain ultimate goals far outside the scope of this project.

\subsubsection{Objective}

This study seeks to develop a high-frequency fully elastic cylindrical shell model, which includes an external coating, fluid-loading and periodic finite-length reinforcing ribs. To achieve this goal, the final model will be built in discrete steps of increasing complexity:

1. Fully-elastic cylindrical shell model with infinitesimally-thin periodic ribs, using simple linear spring stiffener model

2. Fully-elastic cylindrical shell model with finite-length ribs, using linear spring stiffener model

3. Fully-elastic cylindrical shell model with Timoshenko-beam based ribs

4. Fully-elastic cylindrical shell model with beam ribs and external fluid loading

5. Fully-elastic cylindrical shell model with acoustic coating, finite-length beam ribs and fluid loading

Model 1 is referred to as the "base" model. It is an infinitely-long elastic cylindrical shell with ring stiffeners attached to the inner surface periodically along its length. These stiffeners have no thickness, but have a stiffness in all 3 directions; thus they strongly affect the behavior of the system. Model 2 takes the Base model and modifies the ribs to have a finite width, such that each rib transmits forces to and from the shell over a certain area. Model 3 replaces the linear spring stiffness of Model 2 with coupled Timoshenko beam ribs. Beam ribs more accurately describe the motion of a real rib and thus improve the fidelity of the system. Model 4 essentially submerges the beam-rib model in an acoustic fluid, which transmits vibration from the shell as fluid pressure and can be measured. This "scattered" pressure is an important measurement regarding how easily the acoustics of an object can be detected.

The fifth and most advanced model features an acoustic coating encompassing the outer surface and includes all of the additions from versions 1-4; Model 5 meets the requirements set by the sponsor for this study. Scattered pressure, which is defined as the reflected pressure plus the radiated pressure due to shell motion, will also be calculated to determine the effect of the system on the surrounding fluid environment. It is measured at some chosen radial distance from the body. Along with the displacement responses of the shell itself, this acoustic radiation is used to measure system performance. Primary goals for this study are to develop Model 5, validate it with a reference, and determine the performance of the system undergoing 2 types of inputs. It is expected that the addition of the acoustic coating will have a large effect on the acoustic response of the shell and reduce the magnitude of scattered pressure waves measured away from the system. 


\subsubsection{Validation}

Ensuring the quality and accuracy of the models described above requires a process to measure responses against known references. Ideally, this is accomplished with a real experiment, where a system (or approximated system) is tested under desired conditions and the performance is directly measured and compared to the simulation. Another possibility is finite element analysis (FEA), where the desired system is constructed from a large number of simple elements that can describe both the solid and fluid material quite accurately.

For this project, laboratory testing and FEA are not possibilities due to the time and costs required, and both are deemed outside the desired scope. However, there are simpler, approximate models that have been validated with FEA, such as the thin-shell model. Using this as a reference, Models $1-5$ can be configured to match the restrictions required by the thinshell. Therefore, if the new models match the reference then they can be considered properly validated. After this process, certain model parameters, including thickness and excitation frequency, will be adjusted outside the bounds of the reference to determine the system behavior and access performance.

\subsection{Literature Review}

Isotropic cylindrical shells have been studied for many years, and analysis of such systems can be found in textbooks on acoustics and waves in solids [1,2]. Propagation of waves through solids can be quite complex and generally involve partial differential equations; solution of these differential equations can sometimes be solved by separation of variables for systems undergoing linear (low amplitude) motion. Applying elasticity theory to the study of thick cylindrical shells has been derived [3], and this is considered the foundation upon which this study builds. The interaction of acoustic waves with cylindrical shells has been investigated [5], and elastic wave scattering through a solid cylinder with discontinuity has also been analyzed [6].

Adding ring stiffeners to the cylindrical model adds points of reflection where the characteristics change from waves propagating purely as a single longitudinal mode to propagating as multiple reflected waves. This requires the inclusion of multiple longitudinal modes to describe the motion of shell and stiffeners together. Interaction of periodic springs on a beam is one example of this phenomenon and requires changing the solution forms to account for the periodic elements [7]. This was extended to thin cylindrical shells using wave propagation coefficients [8]. Free wave propagation of periodic ring stiffeners on a cylindrical shell was examined using finite elements [9], and a Laplace method was developed to numerically analyze thin shells with periodic ring stiffeners [10]. Incorporating the effect of fluid loading on the acoustic radiation of ring-reinforced shells was considered in the spatial frequency (wavenumber) domain for doubly periodic rings [11]. Thin cylinders supported by ribs with T- 
cross-sections was also investigated [12]. For stiffeners that are wide relative to their periodicity, the combined structure can be assumed to behave as an orthotropic shell. Such system under hydrostatic pressure were solved using Flugge equations of motion [13], and axial factor term analysis was used to solve finite length structures [14]. Derivation of the curved Timoshenko beam rib models was completed using both a dynamic stiffness formulation [15] and couple stress theory [16].

In the spatial wavenumber domain, an orthogonalization technique is used to decouple the infinite summations of modes inside the equations of motion for an infinite elastic plate with periodic masses [17]. This technique was used with periodic, finite-length linear spring ribs on a thin-plate [18] and on a thin-shell cylinder [19]. It was also used on a coated, elastic plate with periodic infinitesimally-thin ribs [20]. Acoustic coatings have found numerous applications in the oceanic and maritime industries. The effect of coatings on the acoustic radiation from a fluidloaded shell was studied for flat plates using finite element methods [21], as well as for infinite cylindrical shells using Donnell-Mushtari equations [22]. 


\section{Chapter 2 Base Model Development}

This chapter focuses on the development of the Base model, described in the Introduction. First the unreinforced elastic shell is derived to establish the elasticity equations and the solution using separation of variables. Effects of periodic ribs including how the displacement field solutions are modified is examined, along with the types of rib models implemented and geometry. Next, the thin-shell reference model is derived, which features the orthogonalization technique used throughout this study, and the base elastic model is then constructed following a similar procedure. Finally, the 2 types of excitations: ring loading and acoustic wave, are introduced.

\subsection{Unreinforced Shell}

\subsubsection{Modeling Unreinforced Shell}

Analysis of fully elastic cylindrical shells begins with the exact equations of elasticity. Unlike the simpler approximate theories, which generally must be restricted to lower frequency motion and thin-wall approximations, elasticity theory is best suited for wide frequency ranges and larger thicknesses. Currently, there are no reinforcing ribs. It is assumed that (1) the cylinder extends infinitely along its axis, (2) all displacements are linear, and (3) the material is homogeneous and isotropic. In cylindrical coordinates, the elasticity, or Navier-Cauchy, equations of motion for a fully elastic body are

$$
\mu \nabla^{2} \boldsymbol{u}(r, \theta, z, t)+(\lambda+\mu) \nabla \nabla \cdot \boldsymbol{u}(r, \theta, z, t)=\rho \frac{\partial^{2} \boldsymbol{u}(r, \theta, z, t)}{\partial t^{2}}
$$

where $\rho$ is material density, $\lambda$ and $\mu$ are Lamé constants and $\mathbf{u}=\left[\begin{array}{lll}u_{r} & u_{\theta} & u_{z}\end{array}\right]^{T}=\left[\begin{array}{lll}w & v & u\end{array}\right]^{T}$ is the cylindrical displacement vector. $\mathrm{w}, \mathrm{v}$ and $\mathrm{u}$ are the radial, circumferential, and axial displacements, respectively. Note that $\nabla^{2}$ refers to the vector Laplace operator. 


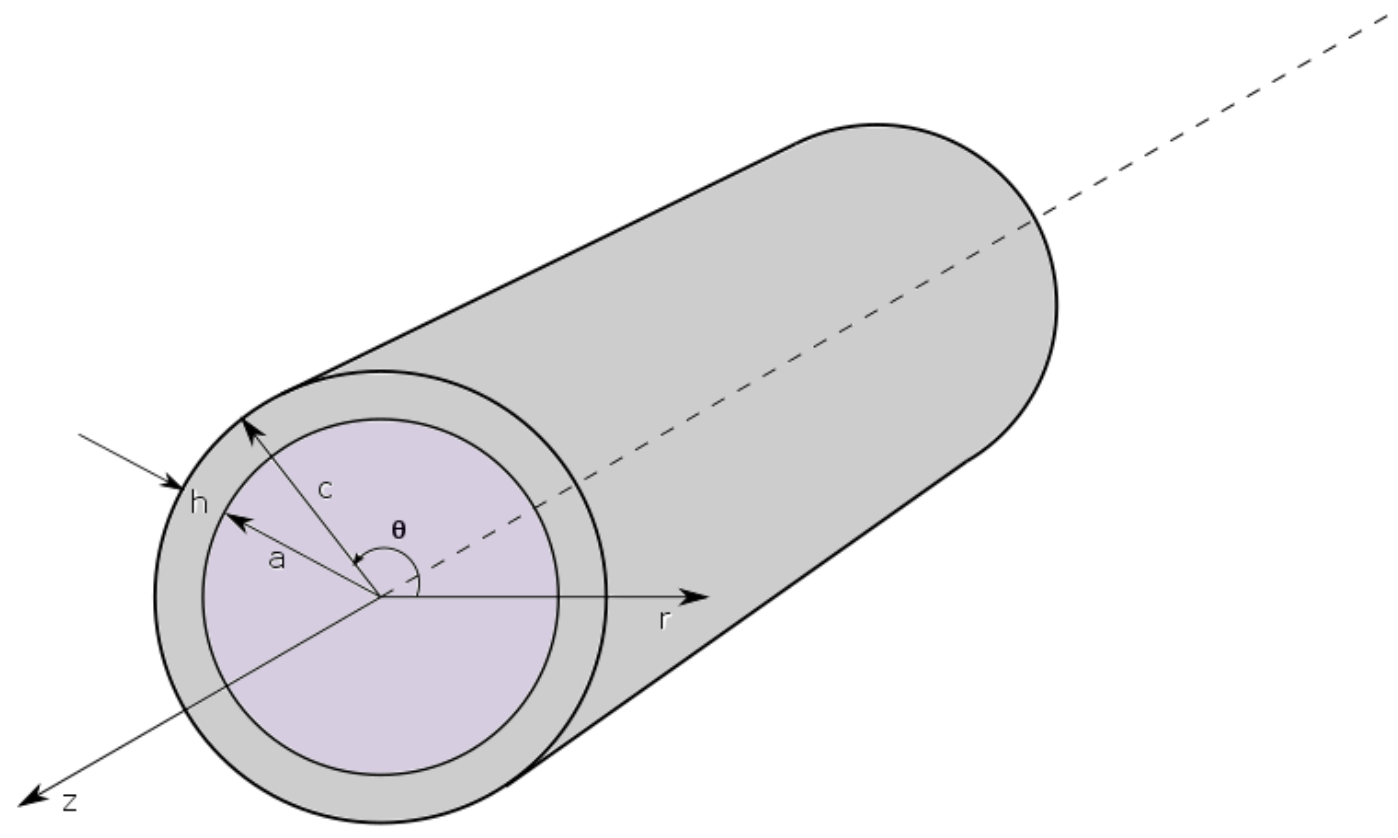

Figure 2.1.1 - Geometry of cylindrical shell

There is a single homogenous shell with two bounding surfaces, the inner surface at radius $r=a$, and the outer surface at $r=c$. Radial normal and radial shear stresses must equal 0 at these surfaces, and this serves as the 6 boundary conditions for the model

$$
\begin{aligned}
& \tau_{r r}=\tau_{r \theta}=\tau_{r z}=0 \quad(r=a) \\
& \tau_{r r}=\tau_{r \theta}=\tau_{r z}=0 \quad(r=c)
\end{aligned}
$$

The stress tensor is given by Hooke's Law of stress

$$
\tau_{i j}=\lambda(\nabla \cdot \boldsymbol{u}) \delta_{i j}+2 \mu \varepsilon_{i j}
$$

where the Kronecker delta $\delta_{i j}=0$ when $\mathrm{i} \neq \mathrm{j}$, and $\varepsilon$ is the strain tensor. Components of the strain tensor are composed of derivatives of $\mathbf{u}$,

$$
\begin{gathered}
\varepsilon_{r r}=\frac{\partial w}{\partial r}, \quad \varepsilon_{\theta \theta}=\frac{1}{r} \frac{\partial v}{\partial \theta}+\frac{w}{r}, \quad \varepsilon_{z z}=\frac{\partial u}{\partial z} \\
\varepsilon_{r \theta}=\frac{1}{2}\left(\frac{1}{r} \frac{\partial w}{\partial \theta}+\frac{\partial v}{\partial r}-\frac{v}{r}\right), \quad \varepsilon_{r z}=\frac{1}{2}\left(\frac{\partial u}{\partial r}+\frac{\partial w}{\partial z}\right), \quad \varepsilon_{\theta z}=\frac{1}{2}\left(\frac{\partial v}{\partial z}+\frac{1}{r} \frac{\partial u}{\partial \theta}\right)
\end{gathered}
$$

Application of the theory for a cylindrical shell, as shown in Figure 2.1.1, follows the procedure outlined by Gazis [3] and Graf [2]. Stresses are calculated at the boundary conditions, and then unknown displacement field constants can be determined. First, the following model assumptions must be listed: (1) the cylinders have infinite spatial extent in the axial direction; (2) deflections of the shells are 3-dimensional and linear. In order to evaluate Equation 2.1.1, the displacement vector $\mathbf{u}$ is written in terms of a dilatational scalar potential, $\phi$, and an equivoluminal vector potential, $\boldsymbol{H}=\left[\begin{array}{lll}H_{r} & H_{\theta} & H_{z}\end{array}\right]^{T}$. 


$$
\boldsymbol{u}=\boldsymbol{\nabla} \Phi+\boldsymbol{\nabla} \times \boldsymbol{H}, \quad \boldsymbol{\nabla} \cdot \boldsymbol{H}=0
$$

This is often referred to as a Helmholtz resolution in field-based physics, and has the property of gauge invariance, meaning that the displacement field $\mathbf{u}$ is invariant to transformations of the potentials (Morse [23]). $\mathbf{H}$ can be chosen to be divergence-less, and this property will allow the 3 components of $\mathbf{u}$ to be determined from the 4 potentials, $\phi$ and $\mathbf{H}$. This property will be used later. Components of $\mathbf{u}$ are expressed in terms of the 4 potentials, taking the gradient and curl in cylindrical coordinates, shown as

$$
\begin{gathered}
w=\frac{\partial \Phi}{\partial r}+\frac{1}{r} \frac{\partial H_{r}}{\partial \theta}-\frac{\partial H_{\theta}}{\partial z} \\
v=\frac{1}{r} \frac{\partial \Phi}{\partial \theta}+\frac{\partial H_{r}}{\partial z}-\frac{\partial H_{z}}{\partial r} \\
u=\frac{\partial \Phi}{\partial z}+\frac{1}{r} \frac{\partial}{\partial r}\left(r H_{\theta}\right)-\frac{1}{r} \frac{\partial H_{r}}{\partial \theta}
\end{gathered}
$$

Inserting the potential-form of $\mathbf{u}$ into the elasticity vector equation gives

$$
\mu \nabla^{2}(\boldsymbol{\nabla} \Phi+\boldsymbol{\nabla} \times \boldsymbol{H})+(\lambda+\mu) \nabla \nabla \cdot(\boldsymbol{\nabla} \Phi+\boldsymbol{\nabla} \times \boldsymbol{H})=\rho \frac{\partial^{2}(\boldsymbol{\nabla} \Phi+\boldsymbol{\nabla} \times \boldsymbol{H})}{\partial t^{2}}
$$

Taking advantage of certain vector identities:

$$
\nabla \cdot(\nabla \times \boldsymbol{H})=0, \quad \nabla \cdot(\nabla \Phi)=\nabla^{2} \Phi, \quad \nabla^{2}(\nabla \Phi)=\nabla\left(\nabla^{2} \Phi\right)
$$

permits rewriting the above and gathering terms into two groups operated on by the gradient and the curl, which is written as

$$
\nabla\left\{(\lambda+2 \mu) \nabla^{2} \Phi-\rho \frac{\partial^{2} \Phi}{\partial t^{2}}\right\}+\nabla \times\left\{\mu \nabla^{2} \boldsymbol{H}-\rho \frac{\partial^{2} \boldsymbol{H}}{\partial t^{2}}\right\}=0
$$

The bracketed terms must vanish for the equation to be satisfied, and this leads to the following independent scalar and vector wave equations

$$
\begin{aligned}
\nabla^{2} \Phi & =\frac{1}{c_{1}^{2}} \frac{\partial^{2} \Phi}{\partial t^{2}}, \quad c_{1}^{2}=\frac{\lambda+2 \mu}{\rho} \\
\nabla^{2} \boldsymbol{H} & =\frac{1}{c_{2}^{2}} \frac{\partial^{2} \boldsymbol{H}}{\partial t^{2}}, \quad c_{2}^{2}=\frac{\mu}{\rho}
\end{aligned}
$$

in which $c_{1}$ and $c_{2}$ represents the scalar dilatational and distortional (rotational) wave speeds. Dilatational waves, sometimes referred to as $\mathrm{P}$-waves or compression waves, cause oscillation in the direction of wave propagation. Rotational waves are sometimes referred to as S-waves, or shear waves, and cause no change in volume. Thus waves transmit through an elastic medium at two distinct speeds. Note that inelastic media, particularly Newtonian fluids like water, do not transmit shear waves. Laplacian operators have a different form for scalar and vector arguments 


$$
\begin{gathered}
\nabla^{2} \Phi=\frac{\partial^{2} \Phi}{\partial r^{2}}+\frac{1}{r} \frac{\partial \Phi}{\partial r}+\frac{1}{r^{2}} \frac{\partial^{2} \Phi}{\partial \theta^{2}}+\frac{\partial^{2} \Phi}{\partial z^{2}} \\
\nabla^{2} \boldsymbol{H}=\left(\nabla^{2} H_{r}-\frac{H_{r}}{r^{2}}-\frac{2}{r^{2}} \frac{\partial H_{\theta}}{\partial \theta}\right) \boldsymbol{e}_{r}+\left(\nabla^{2} H_{\theta}-\frac{H_{\theta}}{r^{2}}+\frac{2}{r^{2}} \frac{\partial H_{r}}{\partial \theta}\right) \boldsymbol{e}_{\boldsymbol{\theta}}+\nabla^{2} H_{z} \boldsymbol{e}_{\boldsymbol{z}}
\end{gathered}
$$

where $\mathbf{e}_{\mathbf{r}}, \mathbf{e}_{\boldsymbol{\theta}}$ and $\mathbf{e}_{\mathbf{z}}$ are the cylindrical unit vectors.

\subsubsection{Evaluating the Wave Equations}

In order to determine the displacement field, $\mathbf{u}$, the potentials must be found using the wave equations. Since the displacements are assumed to be harmonic spatially (z) and temporally, the potential solutions will have the following general form

$$
\begin{aligned}
\Phi & =f(r) \Theta_{\Phi}(\theta) e^{i k z} e^{-i \omega t} \\
H_{r} & =h_{r}(r) \Theta_{r}(\theta) e^{i k z} e^{-i \omega t} \\
H_{\theta} & =h_{\theta}(r) \Theta_{\theta}(\theta) e^{i k z} e^{-i \omega t} \\
H_{z} & =h_{z}(r) \Theta_{z}(\theta) e^{i k z} e^{-i \omega t}
\end{aligned}
$$

where $\omega$ is the frequency, $k$ is the axial wavenumber $f, h_{r}, h_{\theta}$, and $h_{z}$ are unknown functions of $r$, and $\Theta$ is an unknown function of $\theta$. To find $\Theta$, the proposed solutions must be inserted into their respective wave equation. For example, substituting $\phi$ into Equation 2.1.14, and canceling the common exponentials, gives

$$
f^{\prime \prime} \Theta_{\Phi}+\frac{1}{r} f^{\prime} \Theta_{\Phi}+\frac{1}{r^{2}} f \Theta_{\Phi}^{\prime \prime}-k^{2} f \Theta_{\Phi}=-\frac{\omega^{2}}{c_{1}^{2}} f \Theta_{\Phi}
$$

where the dash (') indicates a derivative with respect to the independent variable. Isolating the terms dependent on $\theta$ results in the separated equation, which allows the non- $\theta$ terms to be treated as a constant

$$
\frac{r^{2} f^{\prime \prime}}{f}+\frac{r f^{\prime}}{f}-\left(k^{2}-\frac{\omega^{2}}{c_{1}^{2}}\right) r^{2}=-\frac{\Theta_{\Phi}^{\prime \prime}}{\Theta_{\Phi}}=n^{2}
$$

Requirements on periodic $\Theta_{\Phi}$ ensure that $\Theta(0)=\Theta(2 \pi n)$, which forces $n$ to be an integer. The following single variable equation has the well-known solution

$$
\Theta_{\Phi}^{\prime \prime}+n^{2} \Theta_{\Phi}=0 \rightarrow \Theta_{\Phi}=C_{1} \sin (n \theta)+C_{2} \cos (n \theta)
$$

$\mathrm{n}$ can be thought of as the circumferential mode. A similar solution can be found for the 3 components of $\mathbf{H}: H_{r}, H_{\theta}$ and $H_{z}$. For each, either the sine or cosine term can be discarded. Referring back to the components of $\mathbf{u}$, it is desirable to keep a consistent sinusoid configuration (only sines or only cosines) in each equation. Keeping this in mind, the general solutions now take the form 


$$
\begin{aligned}
\Phi & =f(r) \cos (n \theta) e^{i k z} e^{-i \omega t} \\
H_{r} & =h_{r}(r) \sin (n \theta) e^{i k z} e^{-i \omega t} \\
H_{\theta} & =h_{\theta}(r) \cos (n \theta) e^{i k z} e^{-i \omega t} \\
H_{z} & =h_{z}(r) \sin (n \theta) e^{i k z} e^{-i \omega t}
\end{aligned}
$$

Proceeding in a similar manner, the $r$-dependent functions can be found. Inserting the 4 potentials into their respective wave equations and canceling the common trigonometric and exponential terms, results in the following $r$-dependent equations

$$
\begin{gathered}
\frac{d^{2} f}{d r^{2}}+\frac{1}{r} \frac{d f}{d r}+\left(\alpha^{2}-\frac{n^{2}}{r^{2}}\right) f=0 \\
\frac{d^{2} h_{z}}{d r^{2}}+\frac{1}{r} \frac{d h_{z}}{d r}+\left(\beta^{2}-\frac{n^{2}}{r^{2}}\right) h_{z}=0 \\
\frac{d^{2} h_{r}}{d r^{2}}+\frac{1}{r} \frac{d h_{r}}{d r}+\frac{1}{r^{2}}\left(-n^{2} h_{r}+2 n h_{\theta}-h_{r}\right)-k^{2} h_{r}+\frac{\omega^{2}}{c_{2}^{2}} h_{r}=0 \\
\frac{d^{2} h_{\theta}}{d r^{2}}+\frac{1}{r} \frac{d h_{\theta}}{d r}+\frac{1}{r^{2}}\left(-n^{2} h_{\theta}+2 n h_{r}-h_{\theta}\right)-k^{2} h_{\theta}+\frac{\omega^{2}}{c_{2}^{2}} h_{\theta}=0
\end{gathered}
$$

where the terms $\alpha^{2}=\omega^{2} / c_{1}{ }^{2}-k^{2}$ and $\beta^{2}=\omega^{2} / c_{2}{ }^{2}-k^{2}$. The first two differential equations (in $f$ and $h_{z}$ ) have a special form: Bessel's Equation, which has solutions composed of Bessel functions of order $n$. Due to the makeup of the cylindrical Laplacian, the equations in $r$ and $\theta$ are coupled. These may be solved simultaneously to achieve the same structure by subtracting them

$$
\frac{d^{2}\left(h_{r}-h_{\theta}\right)}{d r^{2}}+\frac{1}{r} \frac{d\left(h_{r}-h_{\theta}\right)}{d r}+\left(\beta^{2}-\frac{(n+1)^{2}}{r^{2}}\right)\left(h_{r}-h_{\theta}\right)=0
$$

and also by adding them

$$
\frac{d^{2}\left(h_{r}+h_{\theta}\right)}{d r^{2}}+\frac{1}{r} \frac{d\left(h_{r}+h_{\theta}\right)}{d r}+\left(\beta^{2}-\frac{(n-1)^{2}}{r^{2}}\right)\left(h_{r}+h_{\theta}\right)=0
$$

The solutions of $f, h_{z},\left(h_{r}-h_{\theta}\right)$, and $\left(h_{r}+h_{\theta}\right)$, are all composed of Bessel functions dependent on $r$, with unknown constants that must be determined

$$
\begin{gathered}
f=A J_{n}(\alpha r)+B Y_{n}(\alpha r) \\
h_{z}=C J_{n}(\beta r)+D Y_{n}(\beta r) \\
h_{r}-h_{\theta}=2 E J_{n+1}(\beta r)+2 F Y_{n+1}(\beta r) \\
h_{r}+h_{\theta}=2 E_{2} J_{n-1}(\beta r)+2 F_{2} Y_{n-1}(\beta r)
\end{gathered}
$$


where $J_{n}$ is a $n^{\text {th }}$ order Bessel function of the first kind, $Y_{n}$ is a $n^{\text {th }}$ order Bessel function of the second kind, and $A, B, C, D, E, F, E_{2}, F_{2}$ are unknown integration constants. $n$ is an integer.

\subsubsection{Bessel Functions}

Bessel functions, $J_{n}(x)$ and $Y_{n}(x)$ for real $x$, have a unique structure that resembles an oscillating sinusoid with amplitude decaying with $1 / \sqrt{ } x$ as the argument $x$ goes to infinity. $J_{n}(0)$ is finite, while $Y_{n}(0)$ diverges to $-\infty$. Plots of the first 3 integer order functions are shown in Figure 2.1.2.
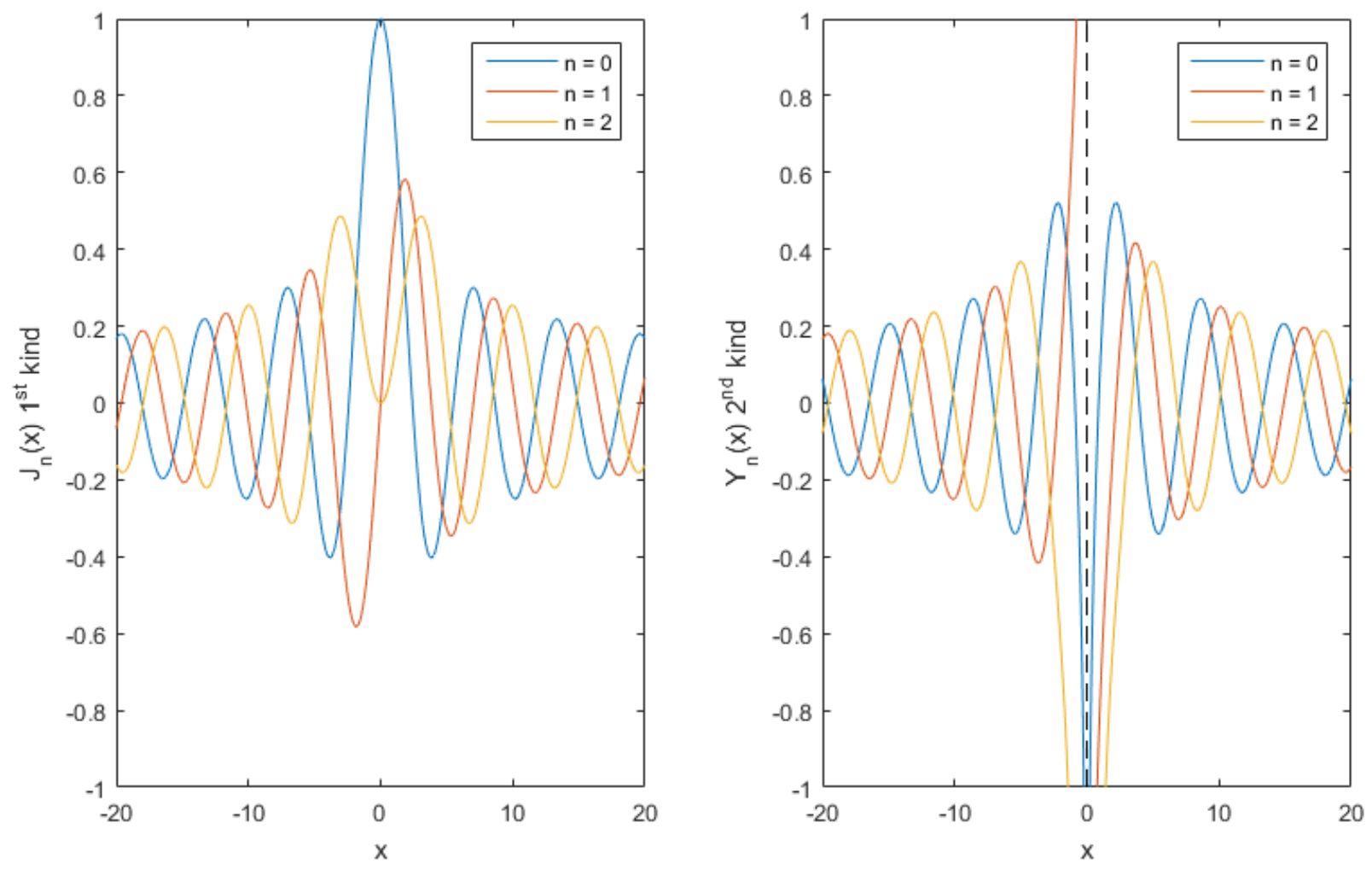

Figure 2.1.2 - Bessel Functions of first (left) and second kind (right) for order $n=0,1,2$

When the argument $\mathrm{x}$ is imaginary, Modified Bessel functions are used, designated $\mathrm{I}_{\mathrm{n}}$ and $\mathrm{K}_{\mathrm{n}}$ for $\mathrm{n}^{\text {th }}$ order modified functions of the first and second kind, respectively. The Modified functions are non-oscillating; $I_{n}(x)$ exponentially grows with $x ; K_{n}(x)$ exponentially decays with $x$, and diverges for $x=0$. Plots of the first 3 integer order functions are shown in Figure 2.1.3. 

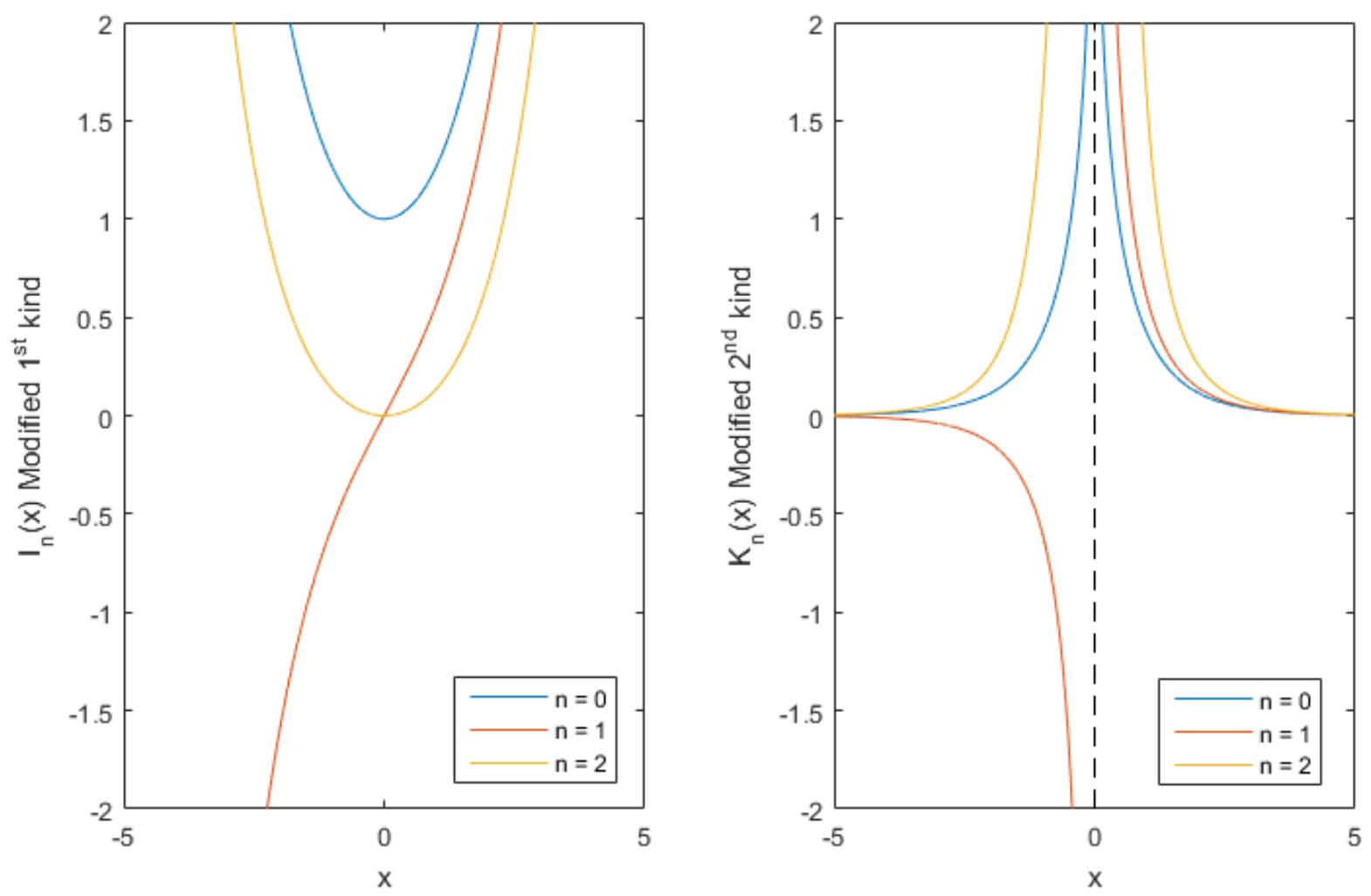

Figure 2.1.3 - Modified Bessel Functions of first kind (left) and second kind (right) for order $\mathrm{n}=0,1,2$

Fortunately, Matlab [24] is able to select the appropriate function to use based on the complex nature of the argument. Bessel functions (modified and unmodified) have helpful properties such as the recurrence relations

$$
\begin{aligned}
& \frac{2 \alpha}{x} Z_{\alpha}(x)=Z_{\alpha-1}(x)+Z_{\alpha+1}(x) \\
& 2 \frac{\partial Z_{\alpha}(x)}{\partial x}=Z_{\alpha-1}(x)-Z_{\alpha+1}(x)
\end{aligned}
$$

where $Z_{\alpha}$ represents a Bessel function of the first or second kind (modified or unmodified). The recurrence relations must be used when a derivative is taken.

\subsubsection{Displacement Solutions}

Currently, there are 8 constants that must be solved using only 6 boundary conditions. However, the aforementioned gauge invariance property of the system allows 2 of the integration constants to be eliminated without loss of generality. Choosing $E_{2}$ and $F_{2}$ to be 0 , ensures that $h_{r}=-h_{\theta}$, leaving 6 unknown coefficients, $A-F$, to be solved with 6 boundary conditions. The components of $\mathbf{u}$ can now be expressed in terms of the potential solutions, with the functions $f, h_{r}$, and $h_{z}$ dependent on $r$ only and still unknown: 


$$
\begin{gathered}
w=\left\{f^{\prime}+\frac{n}{r} h_{z}+k h_{r}\right\} \cos n \theta e^{i(k z-\omega t)} \\
v=\left\{-\frac{n}{r} f+k h_{r}-h_{z}^{\prime}\right\} \sin n \theta e^{i(k z-\omega t)} \\
u=\left\{k f-h_{r}^{\prime}-\frac{n+1}{r} h_{r}\right\} \cos n \theta e^{i(k z-\omega t)}
\end{gathered}
$$

in which the dash (') indicates a derivative with respect to $r$. The bracketed terms are dependent on $r$, but indexed by the circumferential mode $n$. Similarly, the radial normal and shear stresses in the shell can be found by first evaluating Hooke's Law of Stress with the newly found displacements

$$
\begin{gathered}
\tau_{r r}(r, \theta, z, t)=(\lambda+2 \mu) \frac{\partial w}{\partial r}+\frac{\lambda}{r} w+\frac{\lambda}{r} \frac{\partial v}{\partial \theta}+\lambda \frac{\partial u}{\partial z} \\
\tau_{r \theta}(r, \theta, z, t)=\mu \frac{\partial v}{\partial r}-\frac{\mu}{r} v+\frac{\mu}{r} \frac{\partial w}{\partial \theta} \\
\tau_{r z}(r, \theta, z, t)=\mu \frac{\partial w}{\partial z}+\mu \frac{\partial u}{\partial r}
\end{gathered}
$$

and then substituting the potential solutions to get

$$
\begin{gathered}
\tau_{r r}=\left\{-\lambda\left(\alpha^{2}+k^{2}\right) f+2 \mu\left[f^{\prime \prime}+\frac{n}{r}\left(h_{z}^{\prime}-\frac{h_{z}}{r}\right)+k h_{r}^{\prime}\right]\right\} \cos n \theta e^{i(k z-\omega t)} \\
\tau_{r \theta}=\mu\left\{-\frac{2 n}{r}\left(f^{\prime}-\frac{f}{r}\right)-\left(2 h_{z}^{\prime \prime}+\beta^{2} h_{z}\right)-k\left(\frac{n+1}{r} h_{r}-h_{r}^{\prime}\right)\right\} \sin n \theta e^{i(k z-\omega t)} \\
\tau_{r z}=\mu\left\{-2 k f^{\prime}-\frac{n}{r}\left[h_{r}^{\prime}+\left(\frac{n+1}{r}-\beta^{2}+k^{2}\right) h_{r}\right]-\frac{n k}{r} h_{z}\right\} \cos n \theta e^{i(k z-\omega t)}
\end{gathered}
$$

Note that there is a typo in Gazis, Equation 17 for $\tau_{r \theta}:-\beta^{2} h_{z}$ should be $+\beta^{2} h_{z}$. At the boundary conditions: $\left\{\tau_{r r}=\tau_{r \theta}=\tau_{r z}=0 \mid r=a, c\right\}$, these 6 equations and 6 unknown coefficients are grouped into a $6 \times 6$ matrix, AMAT. Since there are no external body forces present, there is no excitation, and thus there is no motion. However, taking the determinant of AMAT $_{\text {M }}$ and setting equal to zero

$$
\operatorname{det}\left(A_{M A T}\right)=0
$$

provides the characteristic equation for the system, dependent upon wavenumber $k$, and circumferential mode, $n$. Gazis discusses numerous simplified motions when setting $n$ or $k$ to zero, but these (and the characteristic equation) are outside the scope of this study. Future models will feature excitation and will focus on forced vibration responses. Still, the use of Bessel functions and method of solving for stresses at the boundary conditions will be needed when stiffeners are added to the elastic model. 
Finally, because the linear displacement solutions 2.1.39 - 2.1.41 must be satisfied for an infinite number of circumferential modes, $(n=0,1,2, \ldots)$, superposition can be applied to achieve the more general solutions

$$
\begin{aligned}
& w(r, \theta, z, t)=e^{i(k z-\omega t)} \sum_{n=0}^{\infty} W_{n}(r) \cos n \theta \\
& v(r, \theta, z, t)=e^{i(k z-\omega t)} \sum_{n=0}^{\infty} V_{n}(r) \sin n \theta \\
& u(r, \theta, z, t)=e^{i(k z-\omega t)} \sum_{n=0}^{\infty} U_{n}(r) \cos n \theta
\end{aligned}
$$

where $W_{n}, V_{n}$, and $U_{n}$ are the radial, tangential and axial wave propagation coefficients. These indexed coefficients are equal to the items inside the curly brackets of 2.1.39-2.1.41, and are functions of $r$.

$$
\begin{gathered}
W_{n}(r)=A_{n}\left[-\alpha J_{n+1}(\alpha r)+\frac{n}{r} J_{n}(\alpha r)\right]+B_{n}\left[-\alpha Y_{n+1}(\alpha r)+\frac{n}{r} Y_{n}(\alpha r)\right]+C_{n} \frac{n}{r} J_{n}(\beta r) \\
\quad+D_{n} \frac{n}{r} Y_{n}(\beta r)+E_{n} i k_{z} J_{n+1}(\beta r)+F_{n} i k_{z} Y_{n+1}(\beta r) \\
V_{n}(r)=-A_{n} \frac{n}{r} J_{n}(\alpha r)-B_{n} \frac{n}{r} Y_{n}(\alpha r)+C_{n}\left[\beta J_{n+1}(\beta r)-\frac{n}{r} J_{n}(\beta r)\right] \\
+D_{n}\left[\beta Y_{n+1}(\beta r)-\frac{n}{r} Y_{n}(\beta r)\right]+E_{n} i k_{z} J_{n+1}(\beta r)+F_{n} i k_{z} Y_{n+1}(\beta r) \\
U_{n}(r)=A_{n} i k_{z} J_{n}(\alpha r)+B_{n} i k_{z} Y_{n}(\alpha r)-E_{n} \beta J_{n}(\beta r)-F_{n} \beta Y_{n}(\beta r)
\end{gathered}
$$

$A_{n}-F_{n}$ are the mode-indexed wave propagation coefficients.

\subsection{Modeling Ribs}

Adding reinforcing stiffeners to a structure can efficiently increase system stiffness without a large increase in system weight. In addition, ribs add stiffness in all directions, but ribs can be configured to affect the system more in certain directions, potentially to make up for structural weaknesses. Ring stiffeners, used to brace cylindrical shells, are a good example of this. Cylindrical shells generally have sufficient torsional strength due to the fact that all of the material is located at the maximum radius, making them more efficient, per weight, than a solid rod. However, radially, shells are hollow and often require additional support, particularly when there is an external pressure differential acting on the system. Placing ring stiffeners periodically along the length of the cylinder can greatly increase the stiffness for the entire length of shell. While 
the axial strength can also be an issue in some cases, particularly in a fuselage with wing attachments, longitudinal ribs can be fitted. For this study, only ring stiffeners are considered. Note that the ribs are assumed to be adhered to the inner surface of the shell body, they are not impregnated into the shell material. Thus, if the ribs were removed, the shell shape would be intact.

\subsubsection{Stiffener Periodicity}

The cylindrical model is assumed to be infinitely long with periodic ring stiffeners spaced a distance $\mathrm{L}$ apart, thus there are an infinite number of stiffeners present in the system. Reinforcement changes the system response significantly relative to an unreinforced cylinder, particularly in the longitudinal direction. Without ribs and damping, waves propagate along the shell length without interruption. However, according to Mead [7], simple harmonic response waves of the form $\mathrm{A}_{0} \mathrm{e}^{\mathrm{ikx}-\mathrm{i} \omega \mathrm{t}}$ cannot exist in a structure with periodic supports. The periodic constraints cause reflections which interfere with the response compared to an unconstrained structure. Nevertheless, if a supported structure is excited by a loading of the form $P_{0} e^{i k x-i \omega t}$, the resulting forced responses will be spatially periodic over the wavelength $2 \pi \mathrm{L} / \sigma$, with phase $\sigma$ $=\mathrm{kL}$. This requires that motion amplitudes must be equal at any two locations a distance $\mathrm{L}$ apart, differing in phase by $\sigma$. Such motion can be represented by an infinite series of harmonic waves with phase differences of $\sigma \pm 2 \pi m$, with integer $m=-\infty, \ldots, 0, \ldots, \infty$. In a simple single degree of freedom case, the transverse displacement response has the form

$$
W(x)=\sum_{m=-\infty}^{\infty} A_{m} e^{\frac{i(\sigma+2 \pi m) x}{L}} e^{-i \omega t}=\sum_{m=-\infty}^{\infty} A_{m} e^{i\left(k+\frac{2 \pi m}{L}\right) x} e^{-i \omega t}
$$

in which $A_{m}$ is an unknown coefficient. For $m=0$, the response matches the excitation, but there are wave components propagating at both faster and slower speeds than the excitation. Also, some components travel in the opposite direction of the excitation, denoted by the negative wavenumber values, which represent reflected waves. It is important to note that the above form characterizes the displacement over a single period $L$, not the entire length of the cylinder, but the infinity of stiffeners in the structure still affect the output. Given that each cylinder length between ribs is equivalent, the entire cylinder length could be constructed, if desired.

Applying the above argument to the cylindrical shell model, the response forms must also be indexed based on the stiffener mode. Displacement fields of an unreinforced cylinder, discussed in section 2.1 , have the following form

$$
\begin{aligned}
& u(r, \theta, z, t)=e^{i k_{z} z} e^{-i \omega t} \sum_{n=0}^{\infty} U_{n}(r) \cos (n \theta) \\
& v(r, \theta, z, t)=e^{i k_{z} z} e^{-i \omega t} \sum_{n=0}^{\infty} V_{n}(r) \sin (n \theta)
\end{aligned}
$$




$$
w(r, \theta, z, t)=e^{i k_{z} z} e^{-i \omega t} \sum_{n=0}^{\infty} W_{n}(r) \cos (n \theta)
$$

where $k_{z}$ is the longitudinal wavenumber, which is constant for a specified input. For the reinforced cylinder shell system, the displacement fields must take into account the interaction of the periodic stiffeners with the shell response,

$$
\begin{aligned}
& u(r, \theta, z, t)=\sum_{m=-\infty}^{\infty} \sum_{n=0}^{\infty} U_{m n}(r) \cos (n \theta) e^{i k_{m} z} e^{-i \omega t} \\
& v(r, \theta, z, t)=\sum_{m=-\infty}^{\infty} \sum_{n=0}^{\infty} V_{m n}(r) \sin (n \theta) e^{i k_{m} z} e^{-i \omega t} \\
& w(r, \theta, z, t)=\sum_{m=-\infty}^{\infty} \sum_{n=0}^{\infty} W_{m n}(r) \cos (n \theta) e^{i k_{m} z} e^{-i \omega t}
\end{aligned}
$$

where $k_{m}=k+2 \pi m / L$ is an indexed longitudinal wavenumber that depends on the rib mode term, $\mathrm{m}$. Displacement responses are now described as series of circumferential modes, $n$, and longitudinal modes, $\mathrm{m}$.

Models for the ring stiffeners must couple the displacements of the shell at the rib location with stresses applied to the shell from the stiffener. There are two separate parts of the stiffener system: the rib-shell connection, and the rib model.

\subsubsection{Rib-Shell Connection}

Connections between the shell model and rib model determine both how stresses are applied to the shell system, and also where the stresses are applied. They are one of the major identifying elements of the model list from section 1.1.2. Essentially, the connection is separate from the rib model, as it is only concerned with transmitting stress into the shell. New rib models can be employed without changes to the connection. There are two types of rib connections:

1.) Delta (infinitesimal width)

2.) Step (finite width)

Delta connections use dirac-delta functions, $\delta(z)$ to transmit stress into the shell. Delta functions have infinitesimal width and extend to infinity

$$
\delta(z)= \begin{cases}\infty, & z=0 \\ 0, & z \neq 0\end{cases}
$$




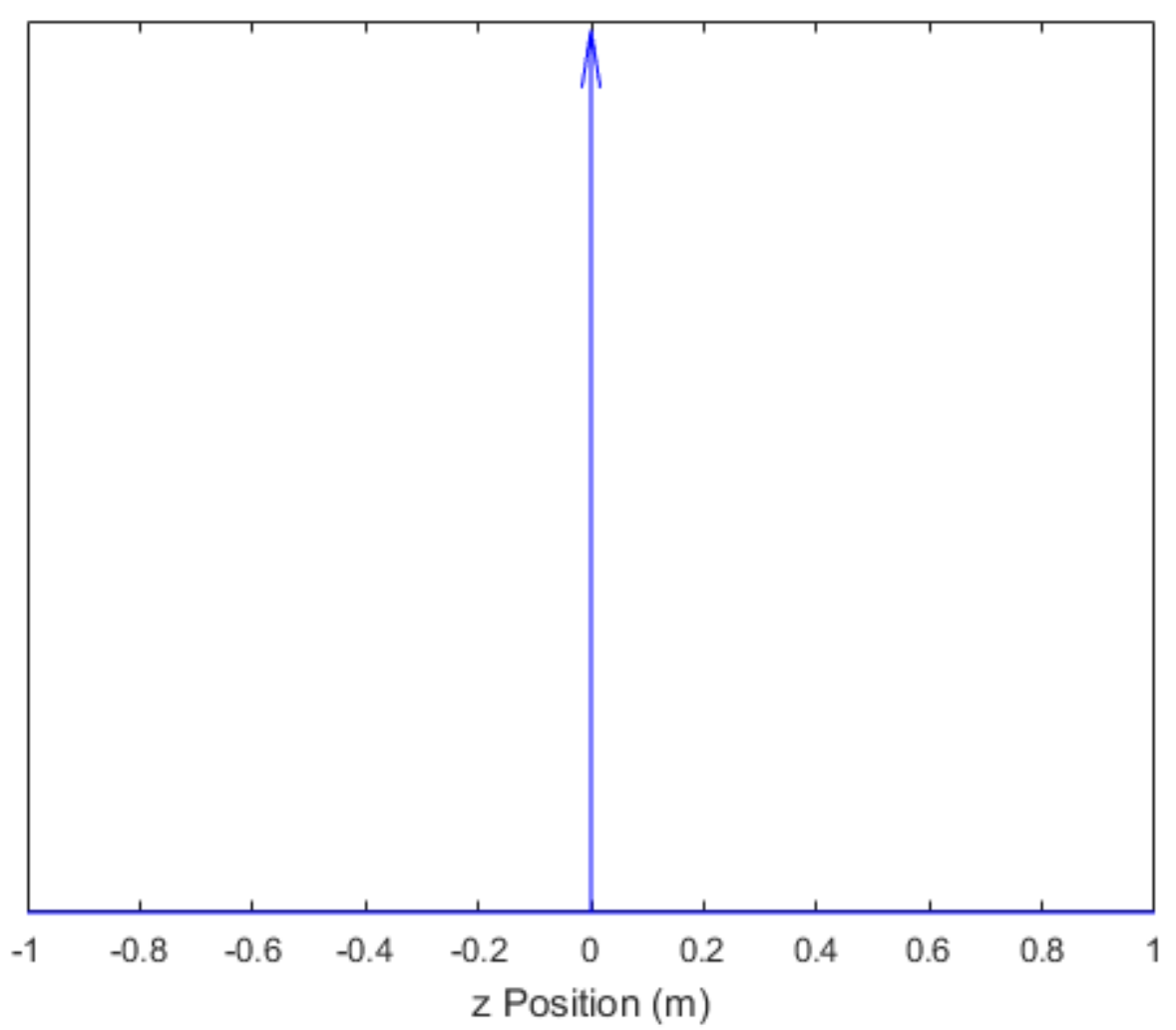

Figure 2.2.1 - Delta function $\delta(\mathrm{z})$

The delta function connection represents an infinitesimally thin rib, which simplifies the system equations. If the rib width, $b$, is small relative to the periodic stiffener spacing, $L,(b<L)$, then this is an appropriate assumption. For larger rib widths, the approximation is less accurate. Note that even if the rib model includes a non-zero width, the connection applies stress to the model only at a single spatial location. The location can be offset to a non-zero location $(z \neq 0)$, but the rib is kept at $z=0$ for this study.

The reinforced shell includes an infinite number of periodic stiffeners, which all influence the motion of the system. Thus there will be an infinite series of delta connections, and this can be written as a Fourier series. Beginning with the definition of an exponential Fourier series

$$
\sum_{s=-\infty}^{\infty} \delta(z-s L)=\sum_{s=-\infty}^{\infty} d_{s} e^{\frac{2 \pi i s z}{L}}
$$

with the Fourier series coefficients, $d_{s}$

$$
d_{s}=\frac{1}{L} \int_{-\frac{L}{2}}^{\frac{L}{2}} \sum_{s=-\infty}^{\infty} \delta(z-s L) e^{-\frac{2 \pi i s z}{L}} d z=\frac{1}{L} \int_{0}^{L} \delta(z) e^{-\frac{2 \pi i s z}{L}} d z=\frac{1}{L}
$$

Thus the Fourier Series for the Delta connectors, also known as a "Dirac Comb", along the cylinder is given as 


$$
\sum_{s=-\infty}^{\infty} \delta(z-s L)=\frac{1}{L} \sum_{s=-\infty}^{\infty} e^{\frac{2 \pi i s z}{L}}
$$

which is differentiable and compatible with the shell model equations. Unfortunately, if the above series is evaluated with a small number of terms, then the series of connections will poorly represent a series of delta functions. Delta connectors have units of $\mathrm{m}^{-1}$.

Step connections use Heaviside functions, $H(z)$, to transmit stress into the shell. Heaviside functions have a step at $z=0$, and are equal to 1 for $z>0$

$$
H(z-b)= \begin{cases}1, & z \geq b \\ 0, & z<b\end{cases}
$$

where $z_{0}$ is an offset. Subtracting an offset Heaviside function from another, $\mathrm{H}(\mathrm{z})-\mathrm{H}(\mathrm{z}-\mathrm{b})$, results in a pulse of specified width, $b$. The finite pulse width is a more accurate representation of real ribs.

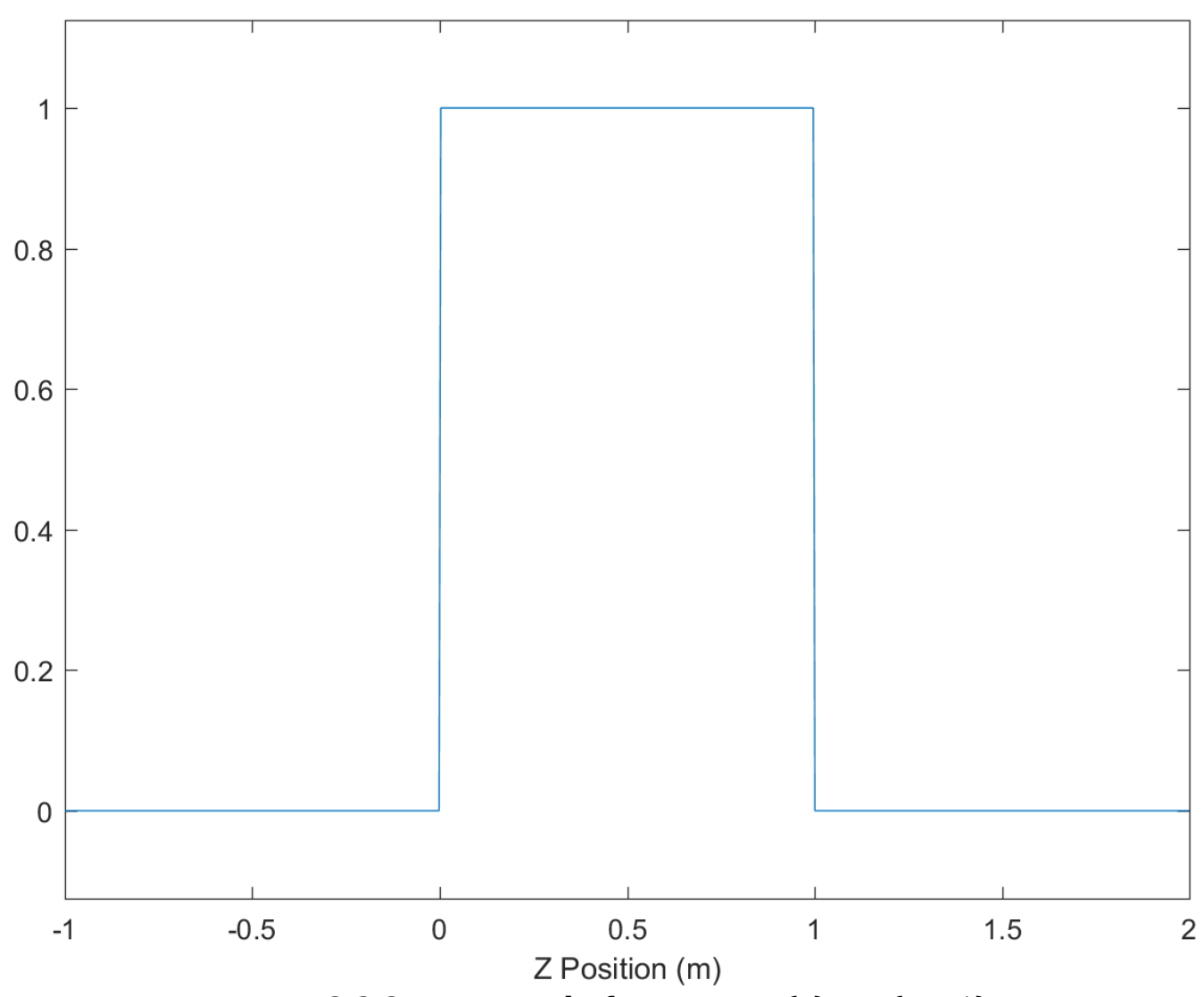

Figure 2.2.2 - Heaviside functions $\mathrm{H}(\mathrm{z})-\mathrm{H}(\mathrm{z}-1)$

Step connections distribute the stiffener stresses over the entire finite rib width, which is equal to the width used in the rib model calculations. As rib widths become smaller the applied stresses from a step connection must converge to the stresses from a delta connection. As with the delta 
functions, it will be necessary later to write the infinite series of step connections as a Fourier series

$$
\sum_{s=-\infty}^{\infty}[H(z-s L)-H(z-b-s L)]=\sum_{s=-\infty}^{\infty} d_{s} e^{\frac{2 \pi i s z}{L}}
$$

It is easiest to first determine the Fourier series for a pulse wave centered about $z=0$, then shift the result $b / 2$ to ensure the pulse starts at $z=0$. Solving for the centered wave Fourier coefficients, $d_{s}{ }^{\prime}$

$$
d_{s}^{\prime}=\frac{1}{L} \int_{-\frac{L}{2}}^{\frac{L}{2}} \sum_{s=-\infty}^{\infty}[H(z-s L)-H(z-b-s L)] e^{-\frac{2 \pi i s z}{L}} d z=\frac{1}{L} \int_{-\frac{b}{2}}^{\frac{b}{2}}(1) e^{-\frac{2 \pi i s z}{L}} d z
$$

which evaluates to

$$
d_{s}^{\prime}=\frac{1}{-2 \pi i s}\left(e^{-\frac{\pi i s b}{L}}-e^{\frac{\pi i s b}{L}}\right)
$$

Performing the $b / 2$ shift on the resultant, represents the Fourier Series of the Step connectors, which starts at $z=0$

$$
\begin{gathered}
\sum_{s=-\infty}^{\infty} d_{s}^{\prime} e^{\frac{2 \pi i s\left(z-\frac{b}{2}\right)}{L}}=\sum_{s=-\infty}^{\infty} \frac{1}{-2 \pi i s}\left(e^{-\frac{\pi i s b}{L}}-e^{\frac{\pi i s b}{L}}\right) e^{-\frac{\pi i s b}{L}} e^{\frac{2 \pi i s z}{L}} \\
=\sum_{s=-\infty}^{\infty} \frac{1}{2 \pi i s}\left(1-e^{\frac{-2 \pi i s b}{L}}\right) e^{\frac{2 \pi i s z}{L}}
\end{gathered}
$$

For the $s=0$ term, the $\lim _{s \rightarrow 0} d_{s}^{\prime}=b / L$. Combining the above, and using $d_{s}$ to represent the $z$-shifted Fourier coefficients, results in

$$
\sum_{s=-\infty}^{\infty}[H(z-s L)-H(z-b-s L)]=\sum_{s=-\infty}^{\infty} d_{s} e^{\frac{2 \pi i s z}{L}}
$$

where

$$
d_{s}=\left\{\begin{array}{cc}
\frac{b}{L}, & s=0 \\
\frac{1-e^{-\frac{2 \pi i s b}{L}}}{2 \pi i s}, & s \neq 0
\end{array}\right.
$$

Equation [2.2.17] is also differentiable and is compatible with the orthogonalization process that will be described later. As with the delta series, if the number of terms, $s$, is too small then the step connections will poorly characterize a series of Heaviside functions. 
Step connectors distribute displacement and force over a finite length, b. Accordingly, when integrating the Step connectors into a model, it is necessary to divide the by the stiffener length, giving units of $\mathrm{m}^{-1}$. This is consistent with both the Delta connectors and ensures that the shell equations are given in terms of stress. Therefore, Step connections are included in stress equations of motion as

$$
\frac{1}{b} \sum_{s=-\infty}^{\infty}[H(z-s L)-H(z-b-s L)]=\frac{1}{b} \sum_{s=-\infty}^{\infty} d_{s} e^{\frac{2 \pi i s z}{L}}
$$

\subsubsection{Rib Models}

Stiffeners must generate stresses based upon the shell displacements, and there are numerous ways to achieve this. The simplest approach is to model the rib as distributed linear springs, with independent stiffness in the axial, tangential and radial directions. Such springs have no frequency dependence, and have no mass. Linear spring models are quite useful because they are easy to implement and the independent stiffnesses can be independently tuned to characterize their effects on the system response.

Modeling the rib structures as beams has many advantages over the simple spring version. The following is a list of effects than can now be considered with beam models:

1.) The beam material has a density and thus inertial effects are included.

2.) Higher order rib motions are possible.

3.) Realistic coupling between displacements is possible.

4.) Better accuracy at higher excitation frequencies.

The Euler-Bernoulli beam model is common and provides all four of the advantages listed above. Compared to more advanced beam models, it requires some restrictive assumptions. Notably, the effects of shear deformation and rotary inertia are ignored. The slope of the beam is approximated as $\psi=\frac{1}{R} \frac{\partial w}{\partial \theta}$. So long as the frequencies of interest are small, these assumptions are usually acceptable. Derivations for curved Euler beam models can be found in Graf [2]. Also, the out-of-plane (axial) motion is independent, while the in-plane motion is coupled. The equations of motion are in the radial and circumferential directions

$$
\begin{gathered}
\frac{E I}{a_{r}^{3}}\left(\frac{\partial^{3} v}{\partial \theta^{3}}-\frac{\partial^{4} w}{\partial \theta^{4}}\right)-\frac{E A}{a_{r}}\left(\frac{\partial v}{\partial \theta}+w\right)+f_{r}=\rho A a_{r} \frac{\partial^{2} w}{\partial t^{2}} \\
\frac{E I}{a_{r}^{3}}\left(\frac{\partial^{2} v}{\partial \theta^{2}}-\frac{\partial^{3} w}{\partial \theta^{3}}\right)+\frac{E A}{a_{r}}\left(\frac{\partial^{2} v}{\partial \theta^{2}}+\frac{\partial w}{\partial \theta}\right)+f_{\theta}=\rho A a_{r} \frac{\partial^{2} v}{\partial t^{2}}
\end{gathered}
$$

in which $E$ is the Young's modulus, $I$ is the moment of area, $a_{r}$ is the in-plane radius of curvature, $A$ is the cross-sectional area (rectangular), $\rho$ is the density of the rib. $f_{r}$ and $f_{\theta}$ are the radial and 
tangential external forces, respectively. The out-of-plane motion is governed by the torsional wave equation

$$
\frac{G J}{a_{r}^{2}} \frac{\partial^{2} \alpha}{\partial \theta^{2}}-\frac{h}{2} f_{z}=\rho J \frac{\partial^{2} \alpha}{\partial t^{2}}, \quad \alpha=\frac{\partial w}{\partial z}
$$

where $J$ is the polar moment of inertia, $G$ is the shear modulus, $f_{z}$ is the external axial force, and $\alpha$ is the rib out-of-plane twist angle. Solving for the rib forces provides the relationship between the rib/shell displacements and the rib forces.

Unfortunately, ignoring shear forces and rotary inertia is a significant disadvantage, so a Timoshenko based model is a natural progression in complexity. Timoshenko beam theory includes the effects of rotary inertia and shear forces, and thus is more accurate than EulerBernoulli, especially at higher frequencies. The angular deflection due to bending $(\psi)$ is included as a state variable, unlike the Euler model, which approximates the bending angle; this requires an additional partial differential equation. Derivations for curved Timoshenko beams are somewhat more involved than those for straight beams, and can be found in the Appendix. The out-of-plane motion is again independent, and the same torsional model is used as before. Equations for the radial, circumferential and in-plane rotational motion are

$$
\begin{gathered}
\frac{E A}{a_{r}}\left(\frac{\partial v}{\partial \theta}-w\right)+\frac{K_{s} G A}{a_{r}}\left(\frac{\partial^{2} w}{\partial \theta^{2}}+\frac{\partial v}{\partial \theta}-a_{r} \frac{\partial \psi}{\partial \theta}\right)+f_{r}=\rho A a_{r} \frac{\partial^{2} w}{\partial t^{2}} \\
\frac{E A}{a_{r}}\left(\frac{\partial^{2} v}{\partial \theta^{2}}-\frac{\partial w}{\partial \theta}\right)-\frac{K_{s} G A}{a_{r}}\left(\frac{\partial w}{\partial \theta}+v-a_{r} \psi\right)+f_{\theta}=\rho A a_{r} \frac{\partial^{2} v}{\partial t^{2}} \\
\frac{E I}{a_{r}} \frac{\partial^{2} \psi}{\partial \theta^{2}}+K_{s} G A\left(\frac{\partial v}{\partial \theta}+w-a_{r} \psi\right)=\rho I a_{r} \frac{\partial^{2} \psi}{\partial t^{2}}
\end{gathered}
$$

$\mathrm{K}_{\mathrm{s}}$ is the cross-section shape factor, dependent on the rib dimensions. In order to be compatible with the shell system, it is necessary to find a decoupled form that eliminates the bending variable, $\psi$. This is done symbolically using the following steps:

For the bending-decoupled radial equation:

1. Solve Equation 2.2.24 for $\psi$, and evaluate: $\frac{\partial \psi}{\partial \theta}, \frac{\partial^{3} \psi}{\partial \theta^{3}}, \frac{\partial^{3} \psi}{\partial \theta \partial \mathrm{t}^{2}}$

2. Differentiate Equation 2.2.26, $\frac{\partial}{\partial \theta}(\ldots)$

3. Input the derivatives from Step 1 into the differentiated Equation from Step 2.

For the bending-decoupled circumferential equation:

1. Solve Equation 2.2.25 for $\psi$, and evaluate: $\frac{\partial^{2} \psi}{\partial \theta^{2}}, \frac{\partial^{2} \psi}{\partial \mathrm{t}^{2}}$ 
2. Input the derivatives from Step 1 into the Equation 2.2.26.

Including the axial equation of motion, reused from the Euler-Bernoulli model, the final Timoshenko beam rib equations are given in the radial direction as

$$
\begin{gathered}
\frac{1}{\mathrm{~K}_{\mathrm{s}} \mathrm{Ga}_{\mathrm{r}}^{3}}\left(\mathrm{IE}^{2} \frac{\partial^{3} \mathrm{v}}{\partial \theta^{3}}-\rho \mathrm{EIa}_{\mathrm{r}}^{2} \frac{\partial^{3} \mathrm{v}}{\partial \theta \partial \mathrm{t}^{2}}+\mathrm{K}_{\mathrm{s}} \mathrm{GIE}^{2} \frac{\partial^{3} \mathrm{v}}{\partial \theta^{3}}-\mathrm{K}_{\mathrm{s}} \mathrm{GI}_{\rho} \mathrm{a}_{\mathrm{r}} \frac{\partial^{3} \mathrm{v}}{\partial \theta \partial \mathrm{t}^{2}}+\mathrm{K}_{\mathrm{s}} \mathrm{GAEa}_{\mathrm{r}}^{2} \frac{\partial \mathrm{v}}{\partial \theta}+\mathrm{IE}^{2} \frac{\partial^{2} \mathrm{w}}{\partial \theta^{2}}\right. \\
-\rho \mathrm{EIa}_{\mathrm{r}}^{2} \frac{\partial^{4} \mathrm{w}}{\partial \theta^{2} \partial \mathrm{t}^{2}}-\rho \mathrm{EIa}_{\mathrm{r}}^{2} \frac{\partial^{2} \mathrm{w}}{\partial \mathrm{t}^{2}}+\mathrm{K}_{\mathrm{s}} \mathrm{GIE} \frac{\partial^{4} \mathrm{w}}{\partial \theta^{4}}+\rho^{2} \mathrm{Ia}_{\mathrm{r}}^{4} \frac{\partial^{4} \mathrm{w}}{\partial \mathrm{t}^{4}}-\rho \mathrm{K}_{\mathrm{s}} \mathrm{GAa}_{\mathrm{r}}^{4} \frac{\partial^{2} \mathrm{w}}{\partial \mathrm{t}^{2}} \\
\left.-\mathrm{K}_{\mathrm{s}} \mathrm{GIa}_{\mathrm{r}}^{2} \rho \frac{\partial^{4} \mathrm{w}}{\partial \theta^{2} \partial \mathrm{t}^{2}}+\mathrm{K}_{\mathrm{s}} \mathrm{GAEa}_{\mathrm{r}}^{2} \mathrm{w}\right)=\mathrm{f}_{\mathrm{r}}
\end{gathered}
$$

in the tangential direction as

$$
\begin{aligned}
\left(\frac{E A}{a_{r}} \frac{\partial v^{2}}{\partial \theta^{2}}-\rho A\right. & \left.a_{r} \frac{\partial^{2} v}{\partial t^{2}}-\frac{\rho I}{a_{r}} \frac{\partial^{2} v}{\partial t^{2}}+\frac{E I}{a_{r}^{3}} \frac{\partial^{2} v}{\partial \theta^{2}}+\frac{\rho^{2} I a_{r}}{K_{s} G} \frac{\partial^{4} v}{\partial t^{4}}+\frac{E^{2} I}{K_{s} G a_{r}^{3}} \frac{\partial^{4} v}{\partial \theta^{4}}-\frac{2 E I \rho}{K_{s} G a_{r}} \frac{\partial^{4} v}{\partial \theta^{2} \partial t^{2}}\right) \\
& +\frac{1}{K_{s} G a_{r}^{3}}\left(I E^{2} \frac{\partial^{3} w}{\partial \theta^{3}}-\rho E I a_{r}^{2} \frac{\partial^{3} w}{\partial \theta \partial t^{2}}+K_{s} G I E \frac{\partial^{3} w}{\partial \theta^{3}}-K_{s} G I \rho a_{r}^{2} \frac{\partial^{3} w}{\partial \theta \partial t^{2}}\right. \\
& \left.+K_{s} G A E a_{r}^{2} \frac{\partial w}{\partial \theta}\right)=f_{\theta}
\end{aligned}
$$

and in the axial direction as

$$
-\frac{2 G J}{h a_{r}^{2}} \frac{\partial^{3} w}{\partial \theta^{2} \partial z}-\frac{2 \rho J}{h} \frac{\partial^{2} w}{\partial t^{2} \partial z}=f_{z}
$$

Shell displacements have the harmonic form shown in Equations 2.2.5 - 2.2.7, and inputting them into the above equations leads to a homogeneous structure that is compatible with the orthogonalization method. This means that the entire radial equation is $\operatorname{composed}$ of $\cos (n \theta)$ terms only instead of mixed cosines and sines, and the tangential equation is composed only of $\sin (n \theta)$ terms. Those equations will be shown later.

Rib stiffeners have many different cross-sections, depending on the desired overall system effect. More complicated cross-sectional shapes require more complicated rib models. Solid, rectangular cross-section ribs are implemented in the model, shown in Figure 2.2.3. 


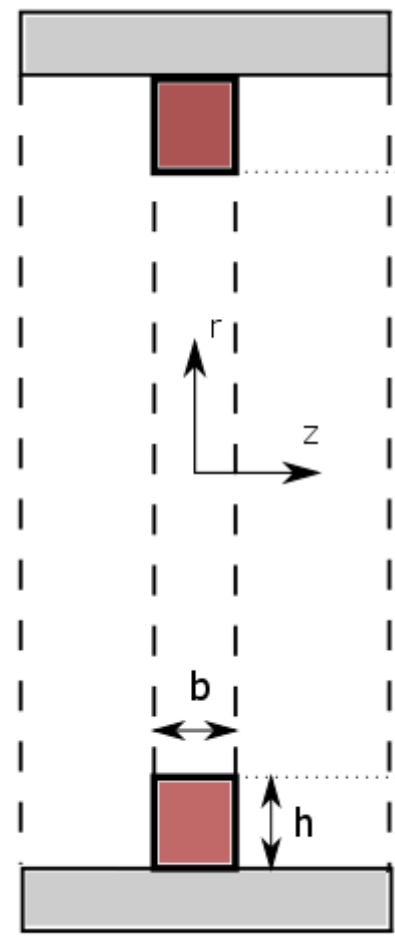

rz Plane

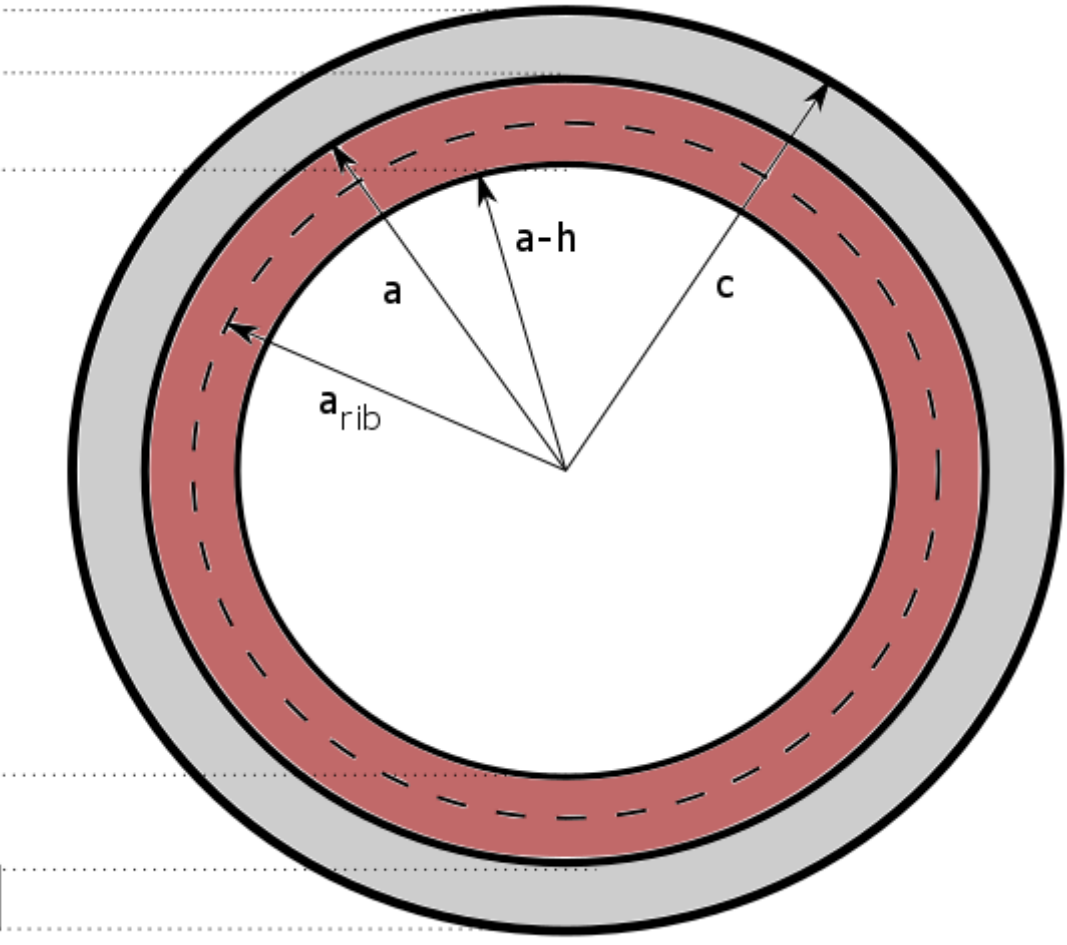

$r \theta$ Plane

Figure 2.2.3 - Rib cross-sectional dimensions shown on the left in the rz-plane, and radial

dimensions shown on the right in the r $\theta$-plane. Rib shown in red, shell in gray

Values of the shape factor, $\mathrm{K}_{\mathrm{s}}$, for rectangular cross-sections are based on the ratio of $\mathrm{h} / \mathrm{b}$, but $5 / 6$ is a valid when $h>2 b$.

\subsection{Thin-Shell Reference}

\subsubsection{Derivation of Reinforced Thin-shell Model}

Before adding reinforcement to the fully elastic model, it is necessary to discuss the ribreinforced thin-shell model, which is used as the reference for validation purposes. The techniques used here to integrate the period stiffeners with the shell model and to orthogonalize the matrix system of equations will also be employed with the elastic model in Chapter 2.4. A derivation of the thin-shell equations of motion, also known as the Donnell equations, will not be included in this study, but can be found in Gould [1]. Instead, this analysis will start with the dynamic formulation of the Donnell shell equations and develop the displacement field responses. The displacement response of a thin cylindrical shell with finite-length ring stiffeners was investigated by Hull [19] and is summarized here. 
The thin-shell system includes a cylindrical shell and periodically spaced ring stiffeners of finite length, as shown in Figure 2.3.1. Donnell shell equations govern the cylinder, while the stiffeners are modeled as independent linear springs, spatially distributed along the stiffener length. As discussed in the Rib Chapter 2.2, finite length ribs require a Step connector type. Also, more complicated beam models are not considered here, but could be used with this system. First the assumptions of the model must be listed: (1) the cylinder extends infinitely along its axis, (2) cylinder displacements are linear and 3-dimensional, (3) cylinder displacements are constant across the shell thickness, (4) ribs are periodically spaced, (5) rib forces are independent and proportional to displacement. Assumption 3 is a necessary condition of a thin-shell model; Assumption 5 is due to the spring rib model.

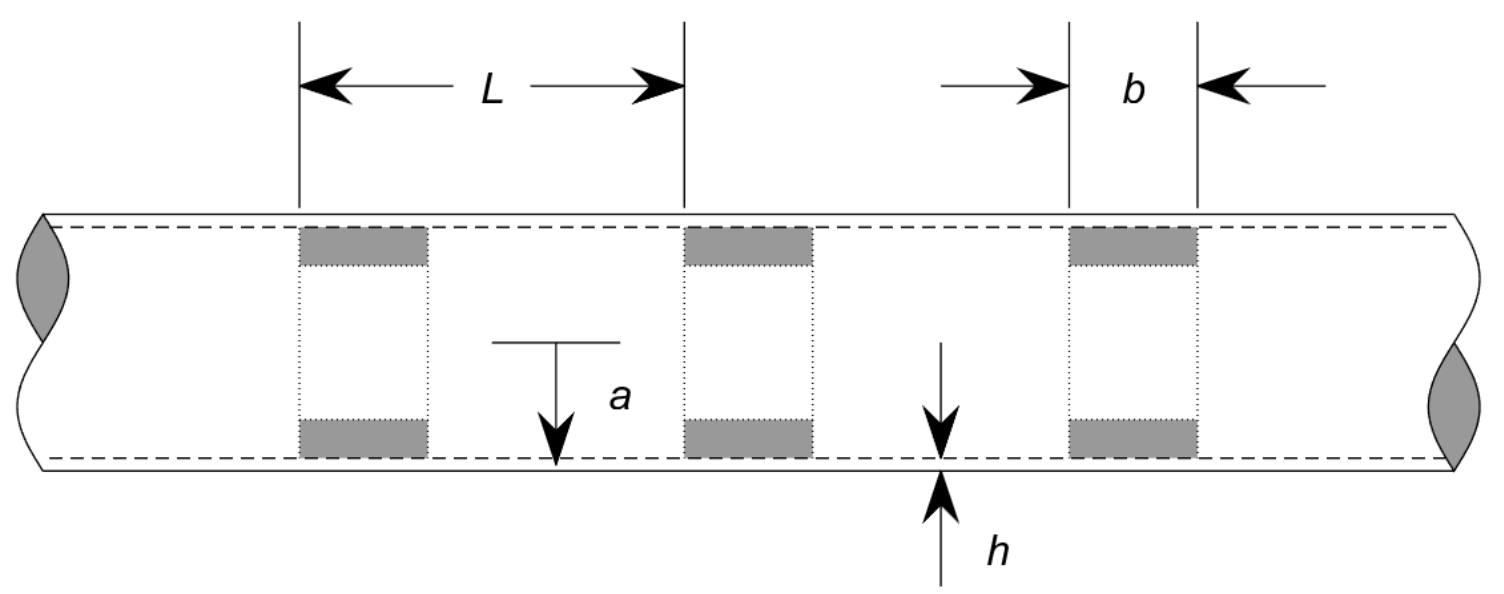

Figure 2.3.1 - Diagram of the thin-shell system geometry including stiffening ribs

Donnell equations (and Figure 2.3.1) only present a single radius, $r=a$; thickness, $h$, is assumed to be symmetric about this nominal radius.

Behavior of the system is governed by the equations of motion, expressed in terms of the displacement vector $\mathbf{u}=[\mathrm{w}(\theta, \mathrm{z}, \mathrm{t}), \mathrm{v}(\theta, \mathrm{z}, \mathrm{t}), \mathrm{u}(\theta, \mathrm{z}, \mathrm{t})]^{\top}$, where $\mathrm{w}, \mathrm{v}$, and $\mathrm{u}$ are the radial, tangential (circumferential), and axial displacement, respectively. Unlike the fully elastic system, these displacements are not functions of $r$, due to assumption 4. The equations include the stresses from the reinforcing ribs and are written in the axial direction as

$$
\begin{gathered}
\rho h \frac{\partial^{2} u}{\partial t^{2}}-\rho h c_{p}^{2} \frac{\partial^{2} u}{\partial z^{2}}-\frac{(1-v) \rho h c_{p}^{2}}{2 a^{2}} \frac{\partial^{2} u}{\partial \theta^{2}}-\frac{(1+v) \rho h c_{p}^{2}}{2 a^{2}} \frac{\partial^{2} v}{\partial z \partial \theta}-\frac{v \rho h c_{p}^{2}}{a} \frac{\partial w}{\partial z} \\
=-\frac{K_{z}}{a b} u \sum_{s=-\infty}^{\infty}[H(z-s L)-H(z-b-s L)]
\end{gathered}
$$

in the tangential direction as 


$$
\begin{gathered}
-\frac{(1+v) \rho h c_{p}^{2}}{2 a} \frac{\partial^{2} u}{\partial z \partial \theta}-\frac{(1-v) \rho h c_{p}^{2}}{2} \frac{\partial^{2} v}{\partial z^{2}}-\frac{\rho h c_{p}^{2}}{a^{2}} \frac{\partial^{2} v}{\partial \theta^{2}}+\rho h \frac{\partial^{2} v}{\partial t^{2}}-\frac{\rho h c_{p}^{2}}{a^{2}} \frac{\partial w}{\partial \theta} \\
\quad=-\frac{K_{t}}{a b} v \sum_{s=-\infty}^{\infty}[H(z-s L)-H(z-b-s L)]
\end{gathered}
$$

and in the radial direction as

$$
\begin{gathered}
\frac{v \rho h c_{p}^{2}}{a} \frac{\partial u}{\partial z}+\frac{\rho h c_{p}^{2}}{a^{2}} \frac{\partial v}{\partial \theta}+\frac{\rho h c_{p}^{2}}{a^{2}} w+\frac{\rho h^{3} c_{p}^{2}}{12} \frac{\partial^{4} w}{\partial z^{4}}+\frac{\rho h c_{p}^{2}}{6 a^{2}} \frac{\partial^{4} w}{\partial z^{2} \partial \theta^{2}}+\frac{\rho h c_{p}^{2}}{12 a^{4}} \frac{\partial^{4} w}{\partial \theta^{4}}+\rho h \frac{\partial^{2} w}{\partial t^{2}} \\
=-\frac{K_{r}}{a b} w \sum_{s=-\infty}^{\infty}[H(z-s L)-H(z-b-s L)]+P_{a}(\theta, z, t)
\end{gathered}
$$

where $b$ is the stiffener length in the axial direction, $L$ is the periodic spacing, $\rho$ is the shell density, $a$ is the shell radius, $h$ is the thickness, $v$ is Poisson's ratio for the shell, $\mathrm{Pa}_{a}$ is the external applied radial pressure. $\mathrm{K}_{2}, \mathrm{~K}_{\mathrm{t}}$, and $\mathrm{K}_{\mathrm{r}}$ are the axial, tangential, and radial linear spring rates. $\mathrm{H}$ is the Heaviside step function, $\mathrm{n}$ is the stiffener index, and $\mathrm{c}_{\mathrm{p}}$ is the plate wave speed

$$
c_{p}^{2}=\frac{E}{\rho\left(1-v^{2}\right)}
$$

The applied external pressure, $\mathrm{P}_{\mathrm{a}}$, is a ring load as discussed in the Excitations Chapter 2.5

$$
P_{a}=P_{0} e^{i k z} e^{-i \omega t}
$$

The same harmonic displacement solutions from the elastic model developed previously can be applied to the thin-shell model, except that the wave propagation constants can be solved directly; they are not functions of other constants. From the Ribs Chapter 2.2, the effect of the periodic ribs on the displacement form requires an additional summation of axial modes $(m)$, resulting in

$$
\begin{gathered}
u(r, \theta, z, t)=\sum_{m=-\infty}^{\infty} \sum_{n=0}^{\infty} U_{m n} \cos (n \theta) e^{i k_{m} z} e^{-i \omega t} \\
v(r, \theta, z, t)=\sum_{m=-\infty}^{\infty} \sum_{n=0}^{\infty} V_{m n} \sin (n \theta) e^{i k_{m} z} e^{-i \omega t} \\
w(r, \theta, z, t)=\sum_{m=-\infty}^{\infty} \sum_{n=0}^{\infty} W_{m n} \cos (n \theta) e^{i k_{m} z} e^{-i \omega t}
\end{gathered}
$$

where $U_{m n}, V_{m n}$, and $W_{m n}$ are the unknown wave propagation coefficients, and $k_{m}$ is the wavenumber indexed by the rib modes

$$
k_{m}=k_{z}+\frac{2 \pi m}{L}
$$


Note that the exponential in time is suppressed for all further developments. Substituting the displacements into the equations of motion leads to

$$
\begin{gathered}
\sum_{m=-\infty}^{\infty} \sum_{n=0}^{\infty}\left\{\left[\rho h c_{p}^{2} k_{m}^{2}+\frac{\rho h c_{p}^{2}(1-v) n^{2}}{2 a^{2}}-\rho h \omega^{2}\right] U_{m n} \cos (n \theta) e^{i k_{m} z}\right. \\
+\left[-\frac{\rho h c_{p}^{2}(1+v) i k_{m} n}{2 a}\right] V_{m n} \cos (n \theta) e^{i k_{m} z} \\
\left.+\left[-\frac{\rho h c_{p}^{2} v i k_{m}}{a}\right] W_{m n} \cos (n \theta) e^{i k_{m} z}\right\} \\
=-\frac{K_{z}}{a b} \sum_{m=-\infty}^{\infty} \sum_{n=0}^{\infty} U_{m n} \cos (n \theta) e^{i k_{m} z} \sum_{s=-\infty}^{\infty}[H(z-s L)-H(z-b-s L)] \\
\sum_{m=-\infty}^{\infty}\left\{\left[\frac { \rho h c _ { p } ^ { 2 } ( 1 + v ) i k _ { m } n ] } { 2 a } \left[U_{m n} \sin (n \theta) e^{i k_{m} z}\right.\right.\right. \\
+\left[\frac{\rho h c_{p}^{2}(1-v) k_{m}^{2}}{2}+\frac{\rho h c_{p}^{2} n^{2}}{a^{2}}-\rho h \omega^{2}\right] V_{m n} \sin (n \theta) e^{i k_{m} z} \\
=-\frac{K_{t}}{a b} \sum_{m=-\infty}^{\infty} \sum_{n=0}^{\infty} V_{m n} \sin (n \theta) e^{i k_{m} z} \sum_{s=-\infty}^{\infty}[H(z-s L)-H(z-b-s L)]
\end{gathered}
$$

$$
\begin{aligned}
\sum_{m=-\infty}^{\infty} & \sum_{n=0}^{\infty}\left\{\left[\frac{\rho h c_{p}^{2} v i k_{m}}{a}\right] U_{m n} \cos (n \theta) e^{i k_{m} z}+\left[\frac{\rho h c_{p}^{2} n}{a^{2}}\right] V_{m n} \cos (n \theta) e^{i k_{m} z}\right. \\
& \left.+\left[\frac{\rho h c_{p}^{2}}{a^{2}}+\frac{\rho h^{3} c_{p}^{2} k_{m}^{4}}{12}+\frac{\rho h^{3} c_{p}^{2} k_{m}^{2} n^{2}}{6 a^{2}}+\frac{\rho h c_{p}^{2} n^{4}}{12 a^{4}}-\rho h \omega^{2}\right] W_{m n} \cos (n \theta) e^{i k_{m} z}\right\} \\
= & -\frac{K_{r}}{a b} \sum_{m=-\infty}^{\infty} \sum_{n=0}^{\infty} W_{m n} \cos (n \theta) e^{i k_{m} z} \sum_{s=-\infty}^{\infty}[H(z-s L)-H(z-b-s L)]+P_{0} e^{i k z}
\end{aligned}
$$

As described in the Ribs Chapter, the Heaviside functions can be represented by a Fourier Series. Replacing the summation of step functions in each equation with a summation of exponentials yields 


$$
\begin{aligned}
& \sum_{m=-\infty}^{\infty} \sum_{n=0}^{\infty}\left\{\left[\rho h c_{p}^{2} k_{m}^{2}+\frac{\rho h c_{p}^{2}(1-v) n^{2}}{2 a^{2}}-\rho h \omega^{2}\right] U_{m n} \cos (n \theta) e^{i k_{m} z}\right. \\
& \left.+\left[-\frac{\rho h c_{p}^{2}(1+v) i k_{m} n}{2 a}\right] V_{m n} \cos (n \theta) e^{i k_{m} z}+\left[-\frac{\rho h c_{p}^{2} v i k_{m}}{a}\right] W_{m n} \cos (n \theta) e^{i k_{m} z}\right\} \\
& =-\frac{K_{z}}{a b} \sum_{m=-\infty}^{\infty} \sum_{n=0}^{\infty} U_{m n} \cos (n \theta) e^{i k_{m} z} \sum_{s=-\infty}^{\infty} d_{s} e^{\frac{2 \pi i s z}{L}} \\
& \sum_{m=-\infty}^{\infty} \sum_{n=0}^{\infty}\left\{+\left[\frac{\left[\frac{\rho h c_{p}^{2}(1-v) k_{m}^{2}}{2 a}+\frac{\rho h c_{p}^{2} n^{2}}{a^{2}}-\rho h \omega^{2}\right] V_{m n} \sin (n \theta) e^{i k_{m} z}}{2}\right\}\right. \\
& =-\frac{K_{t}}{a b} \sum_{m=-\infty}^{\infty} \sum_{n=0}^{\infty} V_{m n} \sin (n \theta) e^{i k_{m} z} \sum_{s=-\infty}^{\infty} d_{s} e^{\frac{2 \pi i s z}{L}} \\
& \sum_{m=-\infty}^{\infty} \sum_{n=0}^{\infty}\left\{\left[\frac{\rho h c_{p}^{2} v i k_{m}}{a}\right] U_{m n} \cos (n \theta) e^{i k_{m} z}+\left[\frac{\rho h c_{p}^{2} n}{a^{2}}\right] V_{m n} \cos (n \theta) e^{i k_{m} z}\right. \\
& \left.+\left[\frac{\rho h c_{p}^{2}}{a^{2}}+\frac{\rho h^{3} c_{p}^{2} k_{m}^{4}}{12}+\frac{\rho h^{3} c_{p}^{2} k_{m}^{2} n^{2}}{6 a^{2}}+\frac{\rho h c_{p}^{2} n^{4}}{12 a^{4}}-\rho h \omega^{2}\right] W_{m n} \cos (n \theta) e^{i k_{m} z}\right\} \\
& =-\frac{K_{r}}{a b} \sum_{m=-\infty}^{\infty} \sum_{n=0}^{\infty} W_{m n} \cos (n \theta) e^{i k_{m} z} \sum_{s=-\infty}^{\infty} d_{s} e^{\frac{2 \pi i s z}{L}}+P_{0} e^{i k z}
\end{aligned}
$$

The n-summations of the last term in each of Equations 2.3.13 - 2.3.15 can be reworked by shifting the index of the term $d_{n}$. A proof of this can be found in the Appendix. For example, in Equation 2.3.13

$$
\sum_{m=-\infty}^{\infty} \sum_{n=0}^{\infty} U_{m n} \cos (n \theta) e^{i k_{m} z} \sum_{s=-\infty}^{\infty} d_{s} e^{\frac{2 \pi i s z}{L}}=\sum_{n=0}^{\infty}\left\{\sum_{s=-\infty}^{\infty} \sum_{m=-\infty}^{\infty} U_{s n} d_{m-s} e^{i k_{m} z}\right\} \cos (n \theta)
$$

Note the change of index on the propagation coefficient. A similar transformation is used on Equations 2.3.14 and 2.3.15. 


$$
\begin{aligned}
& \sum_{m=-\infty}^{\infty} \sum_{n=0}^{\infty}\left\{\left[\rho h c_{p}^{2} k_{m}^{2}+\frac{\rho h c_{p}^{2}(1-v) n^{2}}{2 a^{2}}-\rho h \omega^{2}\right] U_{m n} \cos (n \theta) e^{i k_{m} z}\right. \\
& \left.+\left[-\frac{\rho h c_{p}^{2}(1+v) i k_{m} n}{2 a}\right] V_{m n} \cos (n \theta) e^{i k_{m} z}+\left[-\frac{\rho h c_{p}^{2} v i k_{m}}{a}\right] W_{m n} \cos (n \theta) e^{i k_{m} z}\right\} \\
& =-\frac{K_{z}}{a b} \sum_{n=0}^{\infty}\left\{\sum_{s=-\infty}^{\infty} \sum_{m=-\infty}^{\infty} U_{s n} d_{m-s} e^{i k_{m} z}\right\} \cos (n \theta) \\
& \sum_{m=-\infty}^{\infty} \sum_{n=0}^{\infty}\left\{\left[\frac{\rho h c_{p}^{2}(1+v) i k_{m} n}{2 a}\right] U_{m n} \sin (n \theta) e^{i k_{m} z}\right. \\
& +\left[\frac{\rho h c_{p}^{2}(1-v) k_{m}^{2}}{2}+\frac{\rho h c_{p}^{2} n^{2}}{a^{2}}-\rho h \omega^{2}\right] V_{m n} \sin (n \theta) e^{i k_{m} z} \\
& \left.+\left[\frac{\rho h c_{p}^{2} n}{a^{2}}\right] W_{m n} \sin (n \theta) e^{i k_{m} z}\right\}=-\frac{K_{t}}{a b} \sum_{n=0}^{\infty}\left\{\sum_{s=-\infty}^{\infty} \sum_{m=-\infty}^{\infty} V_{s n} d_{m-s} e^{i k_{m} z}\right\} \sin (n \theta) \\
& \sum_{m=-\infty}^{\infty} \sum_{n=0}^{\infty}\left\{\left[\frac{\rho h c_{p}^{2} v i k_{m}}{a}\right] U_{m n} \cos (n \theta) e^{i k_{m} z}+\left[\frac{\rho h c_{p}^{2} n}{a^{2}}\right] V_{m n} \cos (n \theta) e^{i k_{m} z}\right. \\
& \left.+\left[\frac{\rho h c_{p}^{2}}{a^{2}}+\frac{\rho h^{3} c_{p}^{2} k_{m}^{4}}{12}+\frac{\rho h^{3} c_{p}^{2} k_{m}^{2} n^{2}}{6 a^{2}}+\frac{\rho h c_{p}^{2} n^{4}}{12 a^{4}}-\rho h \omega^{2}\right] W_{m n} \cos (n \theta) e^{i k_{m} z}\right\} \\
& =-\frac{K_{r}}{a b} \sum_{n=0}^{\infty}\left\{\sum_{s=-\infty}^{\infty} \sum_{m=-\infty}^{\infty} W_{s n} d_{m-s} e^{i k_{m} z}\right\} \cos (n \theta)+P_{0} e^{i k z}
\end{aligned}
$$

Finally, the equations of motion have been derived. Currently, the 3 equations of motion are expressed with infinite summations, but the orthogonalization process will now be described to show how these equations can be broken down.

\subsubsection{Orthogonalization}

Using the method of orthogonalization, the equations comprised of infinite summations can be decomposed into an infinite set of indexed equations. This process is analogous to developing a Fourier series, making use of orthogonal harmonic functions, namely trigonometric and exponentials. Multiplying by an orthogonal integer counterpart and then integrating over that period results in 0 except when the counterpart is equal to the original expression. This is described with the following properties of sinusoids

$$
\int_{0}^{2 \pi} \cos n \theta \cos n_{2} \theta d \theta= \begin{cases}1, & n=n_{2} \\ 0, & n \neq n_{2}\end{cases}
$$




$$
\begin{gathered}
\int_{0}^{2 \pi} \sin n \theta \sin n_{2} \theta d \theta= \begin{cases}1, & n=n_{2} \\
0, & n \neq n_{2}\end{cases} \\
\int_{0}^{2 \pi} \cos n \theta \sin n_{2} \theta d \theta=0
\end{gathered}
$$

where $n$ and $n_{2}$ are integers. Only when the integer index $n$ is equal to $n_{2}$ is the integrated product nonzero. Similarly, for exponentials

$$
\int_{0}^{L} e^{i k_{m} z} e^{-i k_{m 2} z}= \begin{cases}L, & k_{m}=k_{m 2} \\ 0, & k_{m} \neq k_{m 2}\end{cases}
$$

where $k_{m}$ and $k_{m 2}$ are integers. These properties are very helpful when incorporated inside an infinite summation because, all summed elements of the summation are zero except when $n=$ $\mathrm{n}_{2}$ and/or $\mathrm{k}_{\mathrm{m}}=\mathrm{k}_{\mathrm{m} 2}$. Putting it all together, the double summation equations can be decomposed by multiplying Equations 2.3.17 and 2.3.19 with $\cos \mathrm{n}_{2} \theta \mathrm{e}^{-\mathrm{ik} \mathrm{k}_{2} \mathrm{z}}$ and Equation 2.3.18 with $\sin \mathrm{n}_{2} \theta \mathrm{e}^{-\mathrm{ik} \mathrm{m}_{2} \mathrm{z}}$, and then integrating $[0,2 \pi]$ on $\theta$ and $[0, \mathrm{~L}]$ on $\mathrm{z}$

$$
\int_{0}^{L} \int_{0}^{2 \pi} \sum_{m=-\infty}^{\infty} \sum_{n=0}^{\infty} \cos n \theta e^{i k_{m} z} \cos n_{2} \theta e^{-i k_{m 2} z} d \theta d z= \begin{cases}L, & n=n_{2}, k_{m}=k_{m 2} \\ 0, & n \neq n_{2}, k_{m} \neq k_{m 2}\end{cases}
$$

Instead of 3 distinct equations made up of infinite summations, the orthogonalization process has decomposed them into an infinite set of $\left(m_{2}, n_{2}\right)$ modal equations. For each individual modal equation, the infinite summation and the respective harmonic function is eliminated. Note that the indexed equations will still be referred to using the $(m, n)$ index rather than the $\left(m_{2}, n_{2}\right)$ index. Orthogonalization can be implemented even when the harmonic function has a constant argument (ie. $\mathrm{e}^{-\mathrm{ikz}}$ ). In this case, the result is still indexed, but is only nonzero for the $0^{\text {th }}$ index $(\mathrm{m}$ $=0$ ) and will include the Kronecker delta function, $\delta_{0 \mathrm{~m}}$.

\subsubsection{Indexed Equations of Motion}

After implementing the orthogonalization process, Equations 2.3.17 - 2.3.19 have been decomposed into an infinite set of $(m, n)$ indexed equations. Each individual equation is expressed as

$$
\begin{gathered}
{\left[\rho h c_{p}^{2} k_{m}^{2}+\frac{\rho h c_{p}^{2}(1-v) n^{2}}{2 a^{2}}-\rho h \omega^{2}\right] U_{m n}-\left[\frac{\rho h c_{p}^{2}(1+v) i k_{m} n}{2 a}\right] V_{m n}} \\
-\left[\frac{\rho h c_{p}^{2} v i k_{m}}{a}\right] W_{m n}=-\frac{K_{z}}{a b} \sum_{s=-\infty}^{\infty} U_{s n} d_{m-s}
\end{gathered}
$$




$$
\begin{gathered}
{\left[\frac{\rho h c_{p}^{2}(1+v) i k_{m} n}{2 a}\right] U_{m n}+\left[\frac{\rho h c_{p}^{2}(1-v) k_{m}^{2}}{2}+\frac{\rho h c_{p}^{2} n^{2}}{a^{2}}-\rho h \omega^{2}\right] V_{m n}+\left[\frac{\rho h c_{p}^{2} n}{a^{2}}\right] W_{m n}} \\
=-\frac{K_{t}}{a b} \sum_{s=-\infty}^{\infty} V_{s n} d_{m-s} \\
{\left[\frac{\rho h c_{p}^{2} v i k_{m}}{a}\right] U_{m n}+\left[\frac{\rho h c_{p}^{2} n}{a^{2}}\right] V_{m n}+\left[\frac{\rho h c_{p}^{2}}{a^{2}}+\frac{\rho h^{3} c_{p}^{2} k_{m}^{4}}{12}+\frac{\rho h^{3} c_{p}^{2} k_{m}^{2} n^{2}}{6 a^{2}}+\frac{\rho h c_{p}^{2} n^{4}}{12 a^{4}}-\rho h \omega^{2}\right] W_{m n}} \\
=-\frac{K_{r}}{a b} \sum_{s=-\infty}^{\infty} W_{s n} d_{m-s}+P_{0} \delta_{m 0, n 0}
\end{gathered}
$$

in which the excitation pressure, $P_{0}$, is only present for the $m=n=0$ index. This implies that $n>$ 0 circumferential modes have no effect on the response under a ring loading. The 3 individual equations can be grouped into a matrix equation that separates the shell dynamics from the stiffener dynamics and external loading. For each $(m, n)$ index, Equations 2.3.25 - 2.3.27 are written as

$$
\boldsymbol{A}_{m n} \boldsymbol{x}_{m n}=-\frac{K_{z}}{a b} \sum_{s=-\infty}^{\infty} \boldsymbol{Z}_{m-s} \boldsymbol{x}_{s n}-\frac{K_{t}}{a b} \sum_{s=-\infty}^{\infty} \boldsymbol{T}_{m-s} \boldsymbol{x}_{s n}-\frac{K_{r}}{a b} \sum_{s=-\infty}^{\infty} \boldsymbol{R}_{m-s} \boldsymbol{x}_{s n}+\boldsymbol{f} \delta_{m 0, n 0}
$$

$\mathbf{A}_{m n}$ is a $3 \times 3$ matrix that describes the shell dynamics, vector $\mathbf{x}_{m n}=\left[U_{m n}, V_{m n}, W_{m n}\right]^{\top}, \mathbf{Z}$, T, and $\mathbf{R}$ are the $3 \times 3$ stiffness matrices in the axial, tangential, and radial directions, and the external force vector, $\mathbf{f}=\left[0,0, P_{0}\right]^{\top}$, is only present for the $(0,0)$ index. The elements of matrix $\mathbf{A}$ are extracted from the indexed equations, and are shown in the Appendix, and the individual stiffener matrices are given as

$$
\boldsymbol{Z}_{\boldsymbol{m}-\boldsymbol{s}}=\left[\begin{array}{ccc}
d_{m-s} & 0 & 0 \\
0 & 0 & 0 \\
0 & 0 & 0
\end{array}\right], \quad \boldsymbol{T}_{\boldsymbol{m}-\boldsymbol{s}}=\left[\begin{array}{ccc}
0 & 0 & 0 \\
0 & d_{m-s} & 0 \\
0 & 0 & 0
\end{array}\right], \quad \boldsymbol{R}_{\boldsymbol{m}-\boldsymbol{s}}=\left[\begin{array}{ccc}
0 & 0 & 0 \\
0 & 0 & 0 \\
0 & 0 & d_{m-s}
\end{array}\right]
$$

\subsubsection{Global Matrix Equation System}

Each indexed $(m, n)$ matrix equation above could be combined into a global matrix equation and solved simultaneously. However, this global system can be assembled in a more efficient manner, as described by Hull [19]. The $n$ index is individually decoupled in each (m, n) matrix equation, unlike the $m$ index, which is coupled inside the stiffener matrices and $s$ summation; axial modes of the shell are effectively coupled by the stiffener forces. Therefore, for each single value of $n$, a global matrix equation of varying $m$ can be constructed and solved. The solutions from this m-global matrix equation is then summed in $\mathrm{n}$ as shown in the original displacement equations 2.3.6 - 2.3.8. For each value of $n$, the $m$-global matrix equation can be expressed as

$$
\widehat{\boldsymbol{A}}_{\boldsymbol{n}} \widehat{\boldsymbol{x}}_{\boldsymbol{n}}=-\frac{K_{z}}{a b} \widehat{\boldsymbol{Z}}_{\boldsymbol{n}} \widehat{\boldsymbol{x}}_{n}-\frac{K_{t}}{a b} \widehat{\boldsymbol{T}}_{\boldsymbol{n}} \widehat{\boldsymbol{x}}_{n}-\frac{K_{r}}{a b} \widehat{\boldsymbol{R}}_{\boldsymbol{n}} \widehat{\boldsymbol{x}}_{\boldsymbol{n}}+\widehat{\boldsymbol{f}}_{\boldsymbol{n}}
$$


where $\widehat{A}_{\boldsymbol{n}}$ is a block diagonal matrix that describes the $\mathrm{n}^{\text {th }}$ circumferential mode shell dynamics, $\widehat{\mathbf{x}}_{\mathbf{n}}$ is the m-indexed vector of displacement coefficients, $\widehat{\mathbf{Z}}_{\mathbf{n}}, \widehat{\mathbf{T}}_{\mathbf{n}}$, and $\widehat{\mathbf{R}}_{\mathbf{n}}$ are the block partitioned matrices composed of the individual $Z_{m}, T_{m}$ and $R_{m}$ stiffener matrices for the $n^{\text {th }}$ mode. The structure of the global rib matrices accounts for the s-summation in 2.3.30. $\hat{\boldsymbol{f}}_{\boldsymbol{n}}$ is the system excitation vector for the $\mathrm{n}^{\text {th }}$ mode.

$$
\begin{aligned}
& \widehat{A}_{n}=\left[\begin{array}{ccccccc}
\ddots & & & \vdots & & & \ddots \\
& A_{-2 n} & 0 & 0 & 0 & 0 & \\
& 0 & A_{-1 n} & 0 & 0 & 0 & \\
& 0 & 0 & A_{0 n} & 0 & 0 & \cdots \\
& 0 & 0 & 0 & A_{1 n} & 0 & \\
\ddots & 0 & 0 & 0 & 0 & A_{2 n} & \\
\ddots & & \vdots & & & \ddots
\end{array}\right], \quad \widehat{x}_{n}=\left[\begin{array}{c}
\vdots \\
x_{-2 n} \\
x_{-1 n} \\
x_{0 n} \\
x_{1 n} \\
x_{2 n} \\
\vdots
\end{array}\right] \\
& \widehat{Z}_{n}=\left[\begin{array}{ccccccc}
\ddots & & & \vdots & & & \ddots \\
& Z_{0} & Z_{-1} & Z_{-2} & Z_{-3} & Z_{-4} & \\
& Z_{1} & Z_{0} & Z_{-1} & Z_{-2} & Z_{-3} & \\
\cdots & Z_{2} & Z_{1} & Z_{0} & Z_{-1} & Z_{-2} & \cdots \\
& Z_{3} & Z_{2} & Z_{1} & Z_{0} & Z_{-1} & \\
& Z_{4} & Z_{3} & Z_{2} & Z_{1} & Z_{0} & \\
\ddots & & & \vdots & & & \ddots
\end{array}\right], \widehat{T}_{n}=\left[\begin{array}{ccccccc}
\ddots & & & & & \\
& T_{0} & T_{-1} & T_{-2} & T_{-3} & T_{-4} & \\
& T_{1} & T_{0} & T_{-1} & T_{-2} & T_{-3} & \\
\cdots & T_{2} & T_{1} & T_{0} & T_{-1} & T_{-2} & \cdots \\
& T_{3} & T_{2} & T_{1} & T_{0} & T_{-1} & \\
& T_{4} & T_{3} & T_{2} & T_{1} & T_{0} & \\
\ddots & & & \vdots & & & \ddots
\end{array}\right], \\
& \widehat{\boldsymbol{R}}_{n}=\left[\begin{array}{ccccccc}
\ddots & & & \vdots & & & \ddots \\
& \boldsymbol{R}_{0} & \boldsymbol{R}_{-1} & \boldsymbol{R}_{-2} & \boldsymbol{R}_{-3} & \boldsymbol{R}_{-4} & \\
& \boldsymbol{R}_{1} & \boldsymbol{R}_{0} & \boldsymbol{R}_{-1} & \boldsymbol{R}_{-2} & \boldsymbol{R}_{-3} & \\
\cdots & \boldsymbol{R}_{2} & \boldsymbol{R}_{1} & \boldsymbol{R}_{0} & \boldsymbol{R}_{-1} & \boldsymbol{R}_{-2} & \cdots \\
& \boldsymbol{R}_{3} & \boldsymbol{R}_{2} & \boldsymbol{R}_{1} & \boldsymbol{R}_{0} & \boldsymbol{R}_{-1} & \\
& \boldsymbol{R}_{4} & \boldsymbol{R}_{3} & \boldsymbol{R}_{2} & \boldsymbol{R}_{1} & \boldsymbol{R}_{\mathbf{0}} & \\
\because & & & \vdots & & & \ddots
\end{array}\right], \quad \widehat{\boldsymbol{f}}_{n}=\left[\begin{array}{c}
\vdots \\
\mathbf{0} \\
\mathbf{0} \\
\boldsymbol{f} \\
\mathbf{0} \\
\mathbf{0} \\
\vdots
\end{array}\right]
\end{aligned}
$$

Note that the $\mathrm{m}$-global stiffener matrices are all rank-deficient, due to the singularity of their component matrices, however, the global A matrix is invertible for real material parameters. The solution for $\hat{\mathbf{x}}_{\mathbf{n}}$ is obtained by solving Equation 2.3.32

$$
\widehat{\boldsymbol{x}}_{\boldsymbol{n}}=\left[\widehat{\boldsymbol{A}}_{\boldsymbol{n}}+\frac{K_{z}}{a b} \widehat{\boldsymbol{Z}}_{\boldsymbol{n}}+\frac{K_{t}}{a b} \widehat{\boldsymbol{T}}_{\boldsymbol{n}}+\frac{K_{r}}{a b} \widehat{\boldsymbol{R}}_{\boldsymbol{n}}\right]^{-1} \widehat{\boldsymbol{f}}_{\boldsymbol{n}}
$$

To find the displacement solutions, $u(r, \theta, z, t), v(r, \theta, z, t)$, and $w(r, \theta, z, t)$, the $n$-mode solutions are combined with 2.3.6-2.3.8. The details of the solution method involve numerous challenges that will be described later.

\subsubsection{Modifications for Delta Rib Connectors}

In the derivation above the thin-shell system featured finite-length ring stiffeners. Step connectors are more general than the Delta connectors, and the derivation follows the work of 
Hull [19] directly. However, if it is desired to use an infinitesimal-length ring stiffener the final equations can be converted quite easily.

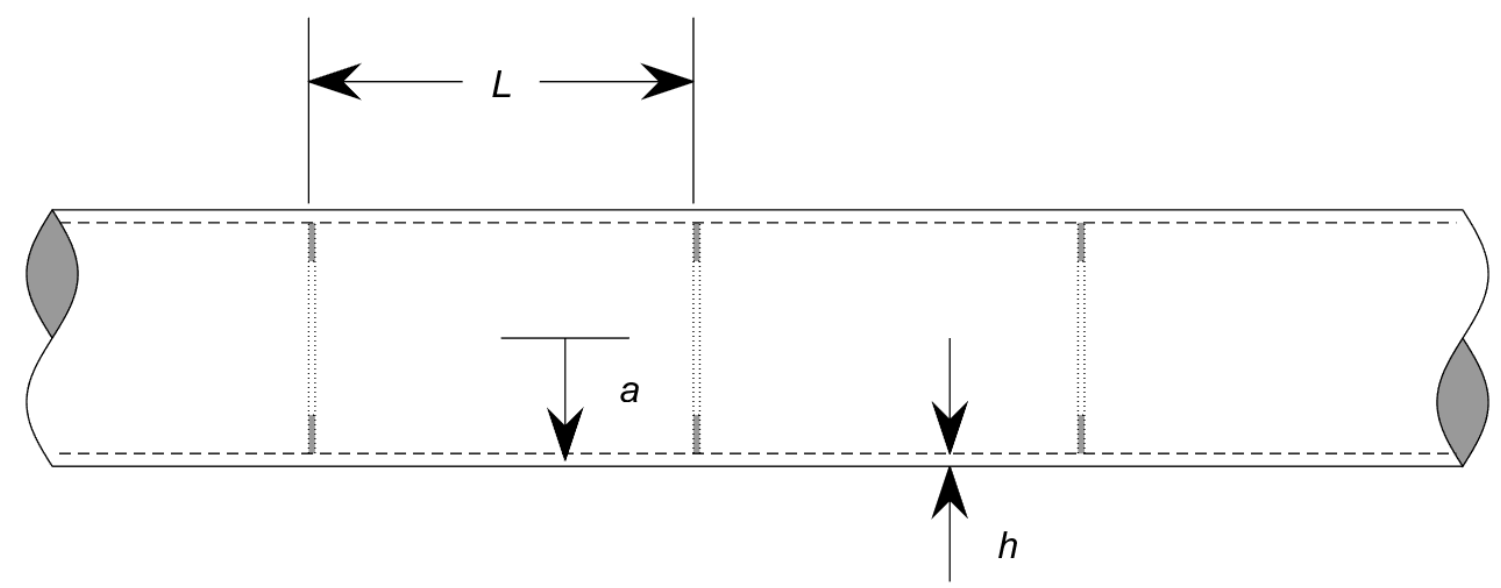

Figure 2.3.2 - Diagram of the thin-shell system geometry with Delta stiffening ribs

Stiffener stresses in the original equation of motion are rewritten as

$$
\begin{gathered}
\tau_{z}=-\frac{K_{z}}{a} u \sum_{s=-\infty}^{\infty} \delta(z-s L), \quad \tau_{\theta}=-\frac{K_{t}}{a} v \sum_{s=-\infty}^{\infty} \delta(z-s L), \\
\tau_{r}=-\frac{K_{z}}{a} w \sum_{s=-\infty}^{\infty} \delta(z-s L)
\end{gathered}
$$

The Fourier Series of the Delta functions was derived in Chapter 2.2, and is given as

$$
\sum_{s=-\infty}^{\infty} \delta(z-s L)=\frac{1}{L} \sum_{s=-\infty}^{\infty} e^{\frac{2 \pi i s z}{L}}
$$

Therefore, it is necessary to replace $d_{m-s}$ in the stiffener matrices, $\mathbf{Z}_{\mathbf{m}-\mathrm{s}}, \mathbf{T}_{\mathbf{m}-\mathrm{s}}, \mathbf{R}_{\mathbf{m}-\mathrm{s}}$ with 1 , and the global matrix equation takes on a similar form

$$
\widehat{\boldsymbol{A}}_{\boldsymbol{n}} \widehat{\boldsymbol{x}}_{\boldsymbol{n}}=-\frac{K_{z}}{a L} \widehat{\boldsymbol{Z}}_{\boldsymbol{n}} \widehat{\boldsymbol{x}}_{n}-\frac{K_{t}}{a L} \widehat{\boldsymbol{T}}_{\boldsymbol{n}} \widehat{\boldsymbol{x}}_{n}-\frac{K_{r}}{a L} \widehat{\boldsymbol{R}}_{\boldsymbol{n}} \widehat{\boldsymbol{x}}_{\boldsymbol{n}}+\widehat{\boldsymbol{f}}_{\boldsymbol{n}}
$$

which can be solved in the same manner as the original system

$$
\widehat{\boldsymbol{x}}_{\boldsymbol{n}}=\left[\widehat{\boldsymbol{A}}_{\boldsymbol{n}}+\frac{K_{z}}{a L} \widehat{\boldsymbol{Z}}_{\boldsymbol{n}}+\frac{K_{t}}{a L} \widehat{\boldsymbol{T}}_{\boldsymbol{n}}+\frac{K_{r}}{a L} \widehat{\boldsymbol{R}}_{\boldsymbol{n}}\right]^{-1} \widehat{\boldsymbol{f}}_{\boldsymbol{n}}
$$




\subsection{Elastic Base Model}

\subsubsection{Derivation of Reinforced Thick-Shell Model}

Adding reinforcement to the fully elastic shell model builds off of the previous 3 chapters, and uses the same solution techniques developed by Hull [19], employed with the thin-shell model. Analysis of the reinforced thick-shell system begins with the Navier-Cauchy elasticity equations, which have been derived in the Appendix. This formulation provides better accuracy at higher frequencies than the shell described in Chapter 2.3, and does not require constant displacement across the shell thickness. Unlike the thin-shell system, however, where the displacement coefficients $(U, V, W)$ were arranged in a 3-dimensional matrix system, the thickshell model requires finding 6 coefficients $(A, B, C, D, E, F)$ within a 6-dimensional system. Displacement field responses, $u(r, \theta, z, t)$, will be found for the entire shell.

The thick-shell system features the same shell as discussed in Chapter 2.2, with the infinitesimal-length periodic ring stiffeners described at the end of Chapter 2.3 (Modifications for Delta Connectors). This rib model features Delta connectors and linear translational springs. More complicated rib systems will be discussed later. Assumptions of the model are: (1) the cylinder extends infinitely along its axis, (2) cylinder displacements are linear and 3-dimensional, (3) shell and rib material is homogenous, isotropic, and symmetric about cylindrical axis, (4) ribs are periodically spaced, (5) rib forces are independent and proportional to displacement. A sidelong cross-sectional view of the cylinder system is shown in Figure 2.4.1.

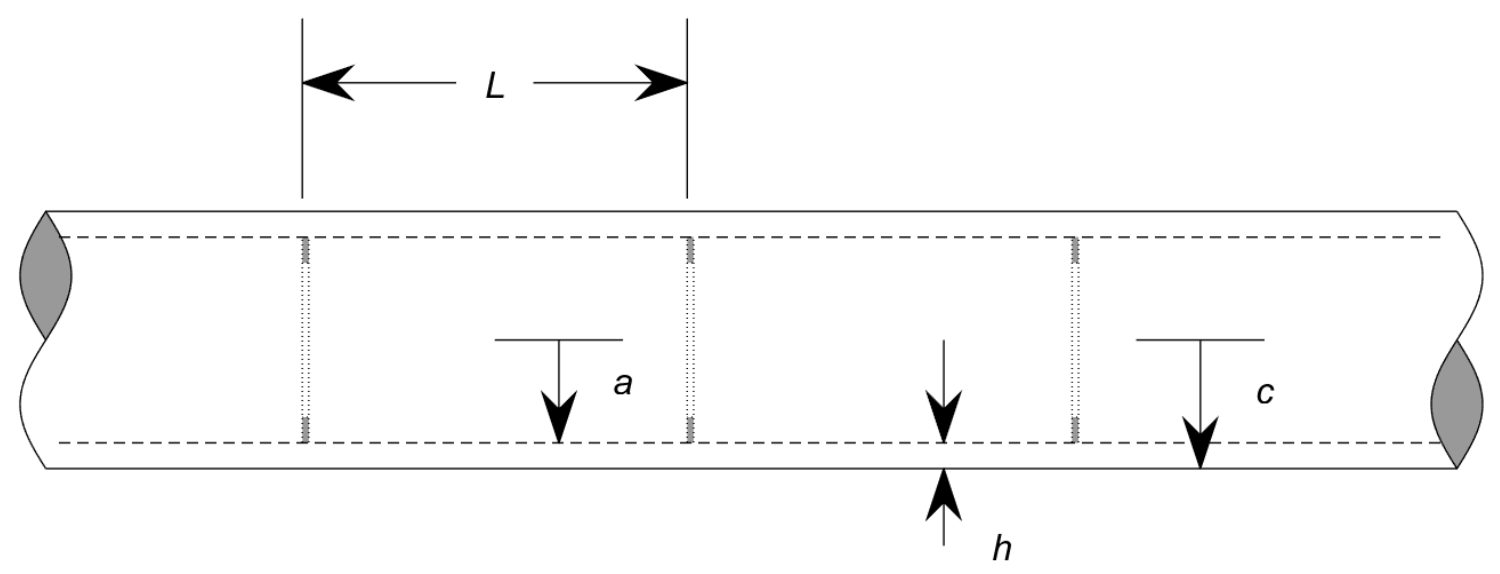

Figure 2.4.1 - Diagram of the thick-shell system geometry including the Delta ribs

System behavior is governed by the vector elasticity equation, expressed in cylindrical coordinates as

$$
\mu \nabla^{2} \boldsymbol{u}(r, \theta, z, t)+(\lambda+\mu) \nabla \nabla \cdot \boldsymbol{u}(r, \theta, z, t)=\rho \frac{\partial^{2} \boldsymbol{u}(r, \theta, z, t)}{\partial t^{2}}
$$


As with the thin-shell system, the effect of the periodic ribs on the displacement form requires an additional summation of axial modes $(m)$, resulting in

$$
\begin{aligned}
& w(r, \theta, z, t)=\sum_{m=-\infty}^{\infty} \sum_{n=0}^{\infty} W_{m n}(r) \cos (n \theta) e^{i k_{m} z} e^{-i \omega t} \\
& v(r, \theta, z, t)=\sum_{m=-\infty}^{\infty} \sum_{n=0}^{\infty} V_{m n}(r) \sin (n \theta) e^{i k_{m} z} e^{-i \omega t} \\
& u(r, \theta, z, t)=\sum_{m=-\infty}^{\infty} \sum_{n=0}^{\infty} U_{m n}(r) \cos (n \theta) e^{i k_{m} z} e^{-i \omega t}
\end{aligned}
$$

where $\mathrm{n}$ is the circumferential mode index, $\mathrm{m}$ is the axial mode index, $\mathrm{U}_{m n}, V_{m n}$, and $W_{m n}$ are the wave propagation coefficients, and $k_{m}$ is the wavenumber indexed by the rib modes

$$
k_{m}=k_{z}+\frac{2 \pi m}{L}
$$

However, these propagation coefficients cannot be solved directly, but are composed of Bessel functions and additional constants. To re-use the coefficient formulation given at the end of the unreinforced elastic shell derivation, it is necessary to make a few changes to include the effects from the periodic ribs. The propagation coefficients are now functions of $r$, and are written as

$$
\begin{gathered}
W_{m n}(r)=A_{m n}\left[-\alpha_{m} J_{n+1}\left(\alpha_{m} r\right)+\frac{n}{r} J_{n}\left(\alpha_{m} r\right)\right]+B_{m n}\left[-\alpha_{m} Y_{n+1}\left(\alpha_{m} r\right)+\frac{n}{r} Y_{n}\left(\alpha_{m} r\right)\right] \\
+C_{m n} \frac{n}{r} J_{n}\left(\beta_{m} r\right)+D_{m n} \frac{n}{r} Y_{n}\left(\beta_{m} r\right)+E_{m n} i k_{m} J_{n+1}\left(\beta_{m} r\right)+F_{m n} i k_{m} Y_{n+1}\left(\beta_{m} r\right) \\
V_{m n}(r)=-A_{m n} \frac{n}{r} J_{n}\left(\alpha_{m} r\right)-B_{m n} \frac{n}{r} Y_{n}\left(\alpha_{m} r\right)+C_{m n}\left[\beta_{m} J_{n+1}\left(\beta_{m} r\right)-\frac{n}{r} J_{n}\left(\beta_{m} r\right)\right] \\
+D_{m n}\left[\beta_{m} Y_{n+1}\left(\beta_{m} r\right)-\frac{n}{r} Y_{n}\left(\beta_{m} r\right)\right]+E_{m n} i k_{m} J_{n+1}\left(\beta_{m} r\right)+F_{m n} i k_{m} Y_{n+1}\left(\beta_{m} r\right) \\
U_{m n}(r)=A_{m n} i k_{m} J_{n}\left(\alpha_{m} r\right)+B_{m n} i k_{m} Y_{n}\left(\alpha_{m} r\right)-E_{m n} \beta_{m} J_{n}\left(\beta_{m} r\right)-F_{m n} \beta_{m} Y_{n}\left(\beta_{m} r\right)
\end{gathered}
$$

where $A_{m n}-F_{m n}$ are the $(m, n)$ mode-indexed unknown coefficients, and the modified dilatational and shear wavenumbers are now indexed by the axial mode, $m$

$$
\alpha_{m}=\sqrt{\left(\frac{\omega}{c_{1}}\right)^{2}-k_{m}^{2}}, \quad \beta_{m}=\sqrt{\left(\frac{\omega}{c_{2}}\right)^{2}-k_{m}^{2}}
$$

Once again the applied external pressure, $\mathrm{P}_{\mathrm{a}}$, is a ring load with constant excitation wavenumber, k. More involved loadings will be discussed later. As before, the temporal harmonic will be suppressed for the remainder of this Chapter.

$$
P_{a}=P_{0} e^{i k z} e^{-i \omega t}
$$




\subsubsection{Evaluating the Boundary Conditions}

In the case of the unreinforced shell with no external loading, the radial normal and radial shear stresses vanished at the bounding inner $(r=a)$ and outer $(r=c)$ surfaces. With the addition of stiffeners at the inner surface and the applied radial excitation at the outer surface, these stress equations must be reformulated. Evaluating Hooke's Law of Stress at $r=$ a gives

$$
\begin{gathered}
\tau_{r r}(a, \theta, z, t)=(\lambda+2 \mu) \frac{\partial w}{\partial r}+\frac{\lambda}{a} w+\frac{\lambda}{a} \frac{\partial v}{\partial \theta}+\lambda \frac{\partial u}{\partial z}=f_{r} \\
\tau_{r \theta}(a, \theta, z, t)=\mu \frac{\partial v}{\partial r}-\frac{\mu}{a} v+\frac{\mu}{a} \frac{\partial w}{\partial \theta}=f_{\theta} \\
\tau_{r z}(a, \theta, z, t)=\mu \frac{\partial w}{\partial z}+\mu \frac{\partial u}{\partial r}=f_{z}
\end{gathered}
$$

where $f_{r}, f_{\theta}$, and $f_{z}$ are the radial, tangential, and axial stresses applied by the stiffeners. Likewise, the stresses at the outer surface $(r=c)$ result in

$$
\begin{gathered}
\tau_{r r}(c, \theta, z, t)=(\lambda+2 \mu) \frac{\partial w}{\partial r}+\frac{\lambda}{c} w+\frac{\lambda}{c} \frac{\partial v}{\partial \theta}+\lambda \frac{\partial u}{\partial z}=P_{a} \\
\tau_{r \theta}(c, \theta, z, t)=\mu \frac{\partial v}{\partial r}-\frac{\mu}{c} v+\frac{\mu}{c} \frac{\partial w}{\partial \theta}=0 \\
\tau_{r z}(c, \theta, z, t)=\mu \frac{\partial w}{\partial z}+\mu \frac{\partial u}{\partial r}=0
\end{gathered}
$$

Substituting the displacements $(2.4 .2-2.4 .4)$ into the 6 stress boundary conditions provides the shell dynamic equations. At the inner surface, Equations 2.4.12 - 2.4.14 become

$$
\begin{gathered}
\sum_{m=-\infty}^{\infty} \sum_{n=0}^{\infty}\left\{(\lambda+2 \mu) \frac{d W_{m n}(a)}{d r}+\frac{\lambda}{a} W_{m n}(a)+\frac{\lambda n}{a} V_{m n}(a)+\lambda i k_{m} U_{m n}(a)\right\} \cos (n \theta) e^{-i k_{m} z} \\
=-\frac{K_{r}}{a} \sum_{m=-\infty}^{\infty} \sum_{n=0}^{\infty} W_{m n} \cos (q \theta) e^{i k_{m} z} \sum_{s=-\infty}^{\infty} \delta(z-s L) \\
\sum_{m=-\infty}^{\infty} \sum_{n=0}^{\infty}\left\{\mu \frac{d V_{m n}(a)}{d r}-\frac{\mu}{a} V_{m n}(a)-\frac{\mu n}{a} W_{m n}(a)\right\} \sin (n \theta) e^{-i k_{m} z} \\
=-\frac{K_{t}}{a} \sum_{m=-\infty}^{\infty} \sum_{n=0}^{\infty} V_{m n} \sin (q \theta) e^{i k_{m} z} \sum_{s=-\infty}^{\infty} \delta(z-s L)
\end{gathered}
$$




$$
\begin{aligned}
& \sum_{m=-\infty}^{\infty} \sum_{n=0}^{\infty}\left\{\mu i k_{m} W_{m n}(a)+\mu \frac{d U_{m n}(a)}{d r}\right\} \cos (n \theta) e^{-i k_{m} z} \\
& =-\frac{K_{z}}{a} \sum_{m=-\infty}^{\infty} \sum_{n=0}^{\infty} U_{m n} \cos (q \theta) e^{i k_{m} z} \sum_{s=-\infty}^{\infty} \delta(z-s L)
\end{aligned}
$$

At the outer boundary surface, Equations (2.4.15 - 2.4.17) become

$$
\begin{aligned}
\sum_{m=-\infty}^{\infty} \sum_{n=0}^{\infty}\left\{(\lambda+2 \mu) \frac{d W_{m n}(c)}{d r}+\frac{\lambda}{c} W_{m n}(c)+\frac{\lambda n}{c} V_{m n}(c)+\lambda i k_{m} U_{m n}(c)\right\} \cos (n \theta) e^{i k_{m} z} \\
=P_{0} e^{i k z} \\
\sum_{m=-\infty}^{\infty} \sum_{n=0}^{\infty}\left\{\mu \frac{d V_{m n}(c)}{d r}-\frac{\mu}{c} V_{m n}(c)-\frac{\mu n}{c} W_{m n}(c)\right\} \sin (n \theta) e^{i k_{m} z}=0 \\
\sum_{m=-\infty}^{\infty} \sum_{n=0}^{\infty}\left\{\mu i k_{m} W_{m n}(c)+\mu \frac{d U_{m n}(c)}{d r}\right\} \cos (n \theta) e^{i k_{m} z}=0
\end{aligned}
$$

which includes the excitation, 2.4.11. As before, the Fourier Series of the Delta function summations are converted into a series of exponentials (2.2.11). Also, the same index shift (Equation 2.3.16) is performed on the stiffener term to rearrange the summation order.

$$
\begin{gathered}
\sum_{m=-\infty}^{\infty} \sum_{n=0}^{\infty}\left\{(\lambda+2 \mu) \frac{d W_{m n}(a)}{d r}+\frac{\lambda}{a} W_{m n}(a)+\frac{\lambda n}{a} V_{m n}(a)+\lambda i k_{m} U_{m n}(a)\right\} \cos (n \theta) e^{-i k_{m} z} \\
=-\frac{K_{r}}{a L} \sum_{n=0}^{\infty}\left\{\sum_{s=-\infty}^{\infty} \sum_{m=-\infty}^{\infty} W_{s n} e^{i k_{m} z}\right\} \cos (n \theta) \\
\sum_{m=-\infty}^{\infty} \sum_{n=0}^{\infty}\left\{\mu \frac{d V_{m n}(a)}{d r}-\frac{\mu}{a} V_{m n}(a)-\frac{\mu n}{a} W_{m n}(a)\right\} \sin (n \theta) e^{-i k_{m} z} \\
=-\frac{K_{t}}{a L} \sum_{n=0}^{\infty}\left\{\sum_{s=-\infty}^{\infty} \sum_{m=-\infty}^{\infty} V_{s n} e^{i k_{m} z}\right\} \sin (n \theta) \\
\sum_{m=-\infty}^{\infty} \sum_{n=0}^{\infty}\left\{\mu i k_{m} W_{m n}(a)+\mu \frac{d U_{m n}(a)}{d r}\right\} \cos (n \theta) e^{-i k_{m} z} \\
=-\frac{K_{z}}{a L} \sum_{n=0}^{\infty}\left\{\sum_{s=-\infty}^{\infty} \sum_{m=-\infty}^{\infty} U_{s n} e^{i k_{m} z}\right\} \cos (n \theta)
\end{gathered}
$$

The system equations $(2.4 .24-2.4 .26$ and $2.4 .21-2.4 .23)$ are now ready for orthogonalization. 


\subsubsection{Mode Decoupled Form}

Following the procedure of Hull [19] with the thin-shell model, the stress equations can be orthogonalized into an infinite set of $(m, n)$ indexed equations. Equations 2.4.21, 2.4.23, 2.4.24, and 2.4.26 are multiplied with $\cos \mathrm{n}_{2} \theta \mathrm{e}^{-\mathrm{i} \mathrm{k}_{\mathrm{m} 2} \mathrm{z}}$, while Equations 2.4 .22 and 2.4 .25 are multiplied with $\sin \mathrm{n}_{2} \theta \mathrm{e}^{-\mathrm{ik} \mathrm{k}_{2} \mathrm{z}}$. Each is then integrated $[0,2 \pi]$ on $\theta$ and $[0, \mathrm{~L}]$ on $z$. The orthogonalization process is described in detail in Chapter 2.3. The infinite summations in $\mathrm{m}$ and $n$ are decoupled into $(m, n)$-indexed equations with the exponentials and sinusoids eliminated. Each set of $6(m, n)$ decoupled equations includes the three radial-normal $\left(\tau_{\mathrm{rr}}\right)$ and radial-shear $\left(\tau_{r \theta}, \tau_{r z}\right)$ stress equations at the inner boundary $(r=a)$

$$
\begin{gathered}
(\lambda+2 \mu) \frac{d W_{m n}(a)}{d r}+\frac{\lambda}{a} W_{m n}(a)+\frac{\lambda n}{a} V_{m n}(a)+\lambda i k_{m} U_{m n}(a)=-\frac{K_{r}}{a L} \sum_{s=-\infty}^{\infty} W_{s n} \\
\mu \frac{d V_{m n}(a)}{d r}-\frac{\mu}{a} V_{m n}(a)-\frac{\mu n}{a} W_{m n}(a)=-\frac{K_{t}}{a L} \sum_{s=-\infty}^{\infty} V_{s n} \\
\mu i k_{m} W_{m n}(a)+\mu \frac{d U_{m n}(a)}{d r}=-\frac{K_{z}}{a L} \sum_{s=-\infty}^{\infty} U_{s n}
\end{gathered}
$$

where the displacement coefficients inside the stiffener summation are indexed with respect to the stiffener index ( $s$ ). The same 3 radial stress equations at the outer boundary $(r=c)$ are

$$
\begin{gathered}
(\lambda+2 \mu) \frac{d W_{m n}(c)}{d r}+\frac{\lambda}{c} W_{m n}(c)+\frac{\lambda n}{c} V_{m n}(c)+\lambda i k_{m} U_{m n}(c)=P_{0} \delta_{m 0, n 0} \\
\mu \frac{d V_{m n}(c)}{d r}-\frac{\mu}{c} V_{m n}(c)-\frac{\mu n}{c} W_{m n}(c)=0 \\
\mu i k_{m} W_{m n}(c)+\mu \frac{d U_{m n}(c)}{d r}=0
\end{gathered}
$$

Combining the displacement formulations $(2.4 .6-2.4 .8)$ with the $(m, n)$ decoupled equations results in the final indexed set of equations. The first 3 equations include the stiffener stresses 


$$
\begin{aligned}
& {\left[\frac{2 \alpha_{m} \mu}{a} J_{n+1}\left(\alpha_{m} a\right)+\left(\frac{2 n(n-1) \mu}{a^{2}}-\alpha_{m}^{2} \lambda-2 \alpha_{m}^{2} \mu-k_{z m}^{2} \lambda\right) J_{n}\left(\alpha_{m} a\right)\right] A_{m n}} \\
& +\left[\frac{2 \alpha_{m} \mu}{a} Y_{n+1}\left(\alpha_{m} a\right)+\left(\frac{2 n(n-1) \mu}{a^{2}}-\alpha_{m}^{2} \lambda-2 \alpha_{m}^{2} \mu-k_{z m}^{2} \lambda\right) Y_{n}\left(\alpha_{m} a\right)\right] B_{m n} \\
& +\left[-\frac{2 n \beta_{m} \mu}{a} J_{n+1}\left(\beta_{m} a\right)+\frac{2 n(n-1) \mu}{a^{2}} J_{n}\left(\beta_{m} a\right)\right] C_{m n} \\
& +\left[-\frac{2 n \beta_{m} \mu}{a} Y_{n+1}\left(\beta_{m} a\right)+\frac{2 n(n-1) \mu}{a^{2}} Y_{n}\left(\beta_{m} a\right)\right] D_{m n} \\
& +\left[-\frac{2 n(n+1) i k_{m} \mu}{a} J_{n+1}\left(\beta_{m} a\right)+2 i k_{m} \beta_{m} \mu J_{n}\left(\beta_{m} a\right)\right] E_{m n} \\
& +\left[-\frac{2 n(n+1) i k_{m} \mu}{a} Y_{n+1}\left(\beta_{m} a\right)+2 i k_{m} \beta_{m} \mu Y_{n}\left(\beta_{m} a\right)\right] F_{m n} \\
& =-\frac{K_{r}}{a L} \sum_{s=-\infty}^{\infty}\left\{\left[-\alpha_{s} J_{n+1}\left(\alpha_{s} a\right)+\frac{n}{r} J_{n}\left(\alpha_{s} a\right)\right] A_{s n}+\left[-\alpha_{s} Y_{n+1}\left(\alpha_{s} a\right)+\frac{n}{a} Y_{n}\left(\alpha_{s} a\right)\right] B_{s n}\right. \\
& \left.+\frac{n}{a} J_{n}\left(\beta_{s} a\right) C_{s n}+\frac{n}{a} Y_{n}\left(\beta_{s} a\right) D_{s n}+i k_{s} J_{n+1}\left(\beta_{s} a\right) E_{s n}+i k_{s} Y_{n+1}\left(\beta_{s} a\right) F_{s n}\right\} \\
& {\left[\frac{2 n \alpha_{m} \mu}{a} J_{n+1}\left(\alpha_{m} a\right)-\frac{2 n(n-1) \mu}{a^{2}} J_{n}\left(\alpha_{m} a\right)\right] A_{m n}} \\
& +\left[\frac{2 n \alpha_{m} \mu}{a} Y_{n+1}\left(\alpha_{m} a\right)-\frac{2 n(n-1) \mu}{a^{2}} Y_{n}\left(\alpha_{m} a\right)\right] B_{m n} \\
& +\left[-\frac{\beta_{m} \mu}{a} J_{n+1}\left(\beta_{m} a\right)+\left(\beta_{m}^{2} \mu-\frac{2 n(n-1) \mu}{a^{2}}\right) J_{n}\left(\beta_{m} a\right)\right] C_{m n} \\
& +\left[-\frac{\beta_{m} \mu}{a} Y_{n+1}\left(\beta_{m} a\right)+\left(\beta_{m}^{2} \mu-\frac{2 n(n-1) \mu}{a^{2}}\right) Y_{n}\left(\beta_{m} a\right)\right] D_{m n} \\
& +\left[-\frac{2(n+1) i k_{m} \mu}{a} J_{n+1}\left(\beta_{m} a\right)+i k_{m} \beta_{m} \mu J_{n}\left(\beta_{m} a\right)\right] E_{m n} \\
& +\left[-\frac{2(n+1) i k_{m} \mu}{a} Y_{n+1}\left(\beta_{m} a\right)+i k_{m} \beta_{m} \mu Y_{n}\left(\beta_{m} a\right)\right] F_{m n} \\
& =-\frac{K_{t}}{a L} \sum_{s=-\infty}^{\infty}\left\{-\frac{n}{a} J_{n}\left(\alpha_{s} a\right) A_{s n}-\frac{n}{a} Y_{n}\left(\alpha_{s} a\right) B_{s n}+\left[\beta_{s} J_{n+1}\left(\beta_{s} a\right)-\frac{n}{a} J_{n}\left(\beta_{s} a\right)\right] C_{s n}\right. \\
& \left.+\left[\beta_{s} Y_{n+1}\left(\beta_{s} a\right)-\frac{n}{a} Y_{n}\left(\beta_{s} a\right)\right] D_{s n}+i k_{s} J_{n+1}\left(\beta_{s} a\right) E_{s n}+i k_{s} Y_{n+1}\left(\beta_{s} a\right) F_{s n}\right\}
\end{aligned}
$$




$$
\begin{gathered}
\\
{\left[-2 i k_{m} \alpha_{m} J_{n+1}\left(\alpha_{m} a\right)+\frac{2 n i k_{m} \mu}{a} J_{n}\left(\alpha_{m} a\right)\right] A_{m n}} \\
+\left[-2 i k_{m} \alpha_{m} Y_{n+1}\left(\alpha_{m} a\right)+\frac{2 n i k_{m} \mu}{a} Y_{n}\left(\alpha_{m} a\right)\right] B_{m n} \\
+\left[\frac{n i k_{m} \mu}{a} J_{n}\left(\beta_{m} a\right)\right] C_{m n}+\left[\frac{n i k_{m} \mu}{a} Y_{n}\left(\beta_{m} a\right)\right] D_{m n} \\
+\left[\mu\left(\beta_{m}^{2}-k_{m}^{2}\right) J_{n+1}\left(\beta_{m} a\right)-\frac{n \beta_{m} \mu}{a} J_{n}\left(\beta_{m} a\right)\right] E_{m n} \\
+\left[\mu\left(\beta_{m}^{2}-k_{m}^{2}\right) Y_{n+1}\left(\beta_{m} a\right)-\frac{n \beta_{m} \mu}{a} Y_{n}\left(\beta_{m} a\right)\right] F_{m n} \\
=-\frac{K_{z}}{a L} \sum_{s=-\infty}^{\infty}\left\{k_{s} J_{n}\left(\alpha_{s} a\right) A_{s n}+i k_{s} Y_{n}\left(\alpha_{s} a\right) B_{s n}-\beta_{s} J_{n}\left(\beta_{s} a\right) E_{s n}-\beta_{s} Y_{n}\left(\beta_{s} a\right) F_{s n}\right\}
\end{gathered}
$$

and the second 3 include the external pressures applied to the outer surface

$$
\begin{gathered}
{\left[\frac{2 \alpha \mu}{c} J_{n+1}\left(\alpha_{m} c\right)+\left(\frac{2 n(n-1) \mu}{c^{2}}-\alpha_{m}^{2} \lambda-2 \alpha_{m}^{2} \mu-k_{z m}^{2} \lambda\right) J_{n}\left(\alpha_{m} c\right)\right] A_{m n}} \\
+\left[\frac{2 \alpha \mu}{c} Y_{n+1}\left(\alpha_{m} c\right)+\left(\frac{2 n(n-1) \mu}{c^{2}}-\alpha_{m}^{2} \lambda-2 \alpha_{m}^{2} \mu-k_{z m}^{2} \lambda\right) Y_{n}\left(\alpha_{m} c\right)\right] B_{m n} \\
+\left[-\frac{2 n \beta_{m} \mu}{c} J_{n+1}\left(\beta_{m} c\right)+\frac{2 n(n-1) \mu}{c^{2}} J_{n}\left(\beta_{m} c\right)\right] C_{m n} \\
+\left[-\frac{2 n \beta_{m} \mu}{c} Y_{n+1}\left(\beta_{m} c\right)+\frac{2 n(n-1) \mu}{c^{2}} Y_{n}\left(\beta_{m} c\right)\right] D_{m n} \\
+\left[-\frac{2 n(n+1) i k_{m} \mu}{c} J_{n+1}\left(\beta_{m} c\right)+2 i k_{m} \beta_{m} \mu J_{n}\left(\beta_{m} c\right)\right] E_{m n} \\
+\left[-\frac{2 n(n+1) i k_{m} \mu}{c} Y_{n+1}\left(\beta_{m} c\right)+2 i k_{m} \beta_{m} \mu Y_{n}\left(\beta_{m} c\right)\right] F_{m n}=P_{0} \delta_{m 0, n 0}
\end{gathered}
$$




$$
\begin{aligned}
& {\left[\frac{2 n \alpha \mu}{c} J_{n+1}\left(\alpha_{m} c\right)-\frac{2 n(n-1) \mu}{a^{2}} J_{n}\left(\alpha_{m} c\right)\right] A_{m n} } \\
+ & {\left[\frac{2 n \alpha \mu}{c} Y_{n+1}\left(\alpha_{m} c\right)-\frac{2 n(n-1) \mu}{c^{2}} Y_{n}\left(\alpha_{m} c\right)\right] B_{m n} } \\
+ & {\left[-\frac{\beta_{m} \mu}{c} J_{n+1}\left(\beta_{m} c\right)+\left(\beta_{m}^{2} \mu-\frac{2 n(n-1) \mu}{c^{2}}\right) J_{n}\left(\beta_{m} c\right)\right] C_{m n} } \\
+ & {\left[-\frac{\beta_{m} \mu}{c} Y_{n+1}\left(\beta_{m} c\right)+\left(\beta_{m}^{2} \mu-\frac{2 n(n-1) \mu}{c^{2}}\right) Y_{n}\left(\beta_{m} c\right)\right] D_{m n} } \\
+ & {\left[-\frac{2(n+1) i k_{m} \mu}{c} J_{n+1}\left(\beta_{m} c\right)+i k_{m} \beta_{m} \mu J_{n}\left(\beta_{m} c\right)\right] E_{m n} } \\
+ & \left.-\frac{2(n+1) i k_{m} \mu}{c} Y_{n+1}\left(\beta_{m} c\right)+i k_{m} \beta_{m} \mu Y_{n}\left(\beta_{m} c\right)\right] F_{m n}=0 \\
+ & {\left[\mu\left(\beta_{m}^{2}-k_{m}^{2}\right) Y_{n+1}\left(\beta_{m} c\right)-\frac{n \beta_{m} \mu}{c} Y_{n}\left(\beta_{m} c\right)\right] F_{m n}=0 } \\
+ & {\left[-2 i k_{m} \alpha_{m} J_{n+1}\left(\alpha_{m} c\right)+\frac{2 n i k_{m} \mu}{c} J_{n}\left(\alpha_{m} c\right)\right] A_{m n} } \\
+ & {\left[\frac{n i k_{m} \mu}{c} J_{n}\left(\beta_{m} c\right)\right] C_{m n}+\left[\frac{n i k_{m} \mu}{c} Y_{n}\left(\beta_{m} c\right)\right] D_{m n} } \\
+ & {\left.\left[\beta_{m}^{2}-k_{m}^{2}\right) J_{n+1}\left(\beta_{m} c\right)-\frac{n \beta_{m} \mu}{c} J_{n}\left(\beta_{m} c\right)\right] E_{m n} } \\
+ & {\left.\left[\alpha_{m} c\right)+\frac{2 n i k_{m} \mu}{c} Y_{n}\left(\alpha_{m} c\right)\right] B_{m n} }
\end{aligned}
$$

The outer surface experiences the ring load excitation only at index $(m, n)=(0,0)$. Higher circumferential modes, $n>0$, have no effect on the response for the ring load excitation.

For each $(m, n)$ index, Equations 2.4.33 - 2.4.38 are grouped together to form a 6dimensional matrix equation, with unknown wave coefficients are grouped together into a vector, $\mathbf{x}$

$$
\boldsymbol{A}_{m n} \boldsymbol{x}_{m n}=-\frac{K_{z}}{a L} \sum_{s=-\infty}^{\infty} \boldsymbol{Z}_{m-s} \boldsymbol{x}_{s n}-\frac{K_{t}}{a L} \sum_{s=-\infty}^{\infty} \boldsymbol{T}_{m-s} \boldsymbol{x}_{s n}-\frac{K_{r}}{a L} \sum_{s=-\infty}^{\infty} \boldsymbol{R}_{m-s} \boldsymbol{x}_{s n}+\boldsymbol{f} \delta_{m 0, n 0}
$$

$A_{m n}$ is a $6 \times 6$ matrix that describes the shell dynamics for the $(m, n)$ mode and is shown in the Appendix. $\mathbf{x}_{\mathrm{mn}}$ is the vector of wave coefficients

$$
\boldsymbol{x}_{\boldsymbol{m n}}=\left[A_{m n}, B_{m n}, C_{m n}, D_{m n}, E_{m n}, F_{m n}\right]^{T}
$$


$\mathbf{Z}, \mathbf{T}$, and $\mathbf{R}$ are the $6 \times 6$ stiffness matrices in the axial, tangential, and radial directions, and the external force vector, $\mathbf{f}$, is only present for the $(0,0)$ index. Each stiffener matrix has a single nonzero row

$$
\begin{gathered}
\boldsymbol{R}_{\boldsymbol{m}-\boldsymbol{s}}(1,1: 6)=\left[\left(-\alpha_{s} J_{n+1}\left(\alpha_{s} a\right)+\frac{n}{r} J_{n}\left(\alpha_{s} a\right)\right), \quad\left(-\alpha_{s} Y_{n+1}\left(\alpha_{s} a\right)+\frac{n}{r} Y_{n}\left(\alpha_{s} a\right)\right), \ldots\right. \\
\left.\frac{n}{a} J_{n}\left(\beta_{s} a\right), \quad i \frac{n}{a} Y_{n}\left(\beta_{s} a\right), \quad i k_{s} J_{n+1}\left(\beta_{s} a\right), \quad i k_{s} Y_{n+1}\left(\beta_{s} a\right)\right] \\
\boldsymbol{T}_{\boldsymbol{m}-\boldsymbol{s}}(2,1: 6)=\left[-\frac{n}{a} J_{n}\left(\alpha_{s} a\right), \quad-\frac{n}{a} Y_{n}\left(\alpha_{s} a\right), \quad\left(\beta_{s} J_{n+1}\left(\beta_{s} a\right)-\frac{n}{a} J_{n}\left(\beta_{s} a\right)\right), \ldots\right. \\
\left.\left(\beta_{s} Y_{n+1}\left(\beta_{s} a\right)-\frac{n}{a} Y_{n}\left(\beta_{s} a\right)\right), \quad i k_{s} J_{n+1}\left(\beta_{s} a\right), \quad i k_{s} J_{n+1}\left(\beta_{s} a\right)\right] \\
\boldsymbol{Z}_{\boldsymbol{m}-\mathbf{s}}(3,1: 6)=\left[k_{s} J_{n}\left(\alpha_{s} a\right), \quad i k_{s} Y_{n}\left(\alpha_{s} a\right), \quad 0, \quad 0, \quad-\beta_{s} J_{n}\left(\beta_{s} a\right), \quad-\beta_{s} Y_{n}\left(\beta_{s} a\right)\right]
\end{gathered}
$$

For the ring load considered in this current system, the excitation vector is specified as

$$
\mathbf{f}=\left[0,0,0, \mathrm{P}_{0}, 0,0\right]^{\mathrm{T}}
$$

\subsubsection{Global Matrix Equation System}

Following the procedure used with the thin-shell system, a global matrix equation of varying $m$ is constructed for each circumferential mode, $n$. Note that each $(m, n)$ stiffener matrix only has a single value of $n$, so the circumferential mode is still totally decoupled. Specifically, the global matrix equation matches Equation 2.3.35. For each value of $n$, the m-global matrix equation can be expressed as

$$
\widehat{\boldsymbol{A}}_{\boldsymbol{n}} \widehat{\boldsymbol{x}}_{\boldsymbol{n}}=-\frac{K_{z}}{a L} \widehat{\boldsymbol{Z}}_{\boldsymbol{n}} \widehat{\boldsymbol{x}}_{n}-\frac{K_{t}}{a L} \widehat{\boldsymbol{T}}_{\boldsymbol{n}} \widehat{\boldsymbol{x}}_{n}-\frac{K_{r}}{a L} \widehat{\boldsymbol{R}}_{\boldsymbol{n}} \widehat{\boldsymbol{x}}_{\boldsymbol{n}}+\widehat{\boldsymbol{f}}_{\boldsymbol{n}}
$$

where $\widehat{\mathbf{A}}_{\mathrm{n}}$ is the block diagonal matrix that describes the $\mathrm{n}^{\text {th }}$ circumferential mode shell dynamics, $\widehat{\mathbf{x}}_{\mathbf{n}}$ is the m-indexed vector of displacement coefficients, $\widehat{\mathbf{Z}}_{\mathbf{n}}, \widehat{\mathbf{T}}_{\mathbf{n}}, \widehat{\mathbf{R}}_{\mathbf{n}}$ are the global matrices comprised of the individual $\mathbf{Z}_{m-s}, \mathbf{T}_{m-s}$ and $\mathbf{R}_{m-s}$ stiffener matrices for the $n^{\text {th }}$ mode. $\hat{\mathbf{f}}_{\mathbf{n}}$ is the system excitation vector, with nonzero elements only at $m=0$.

$$
\widehat{A}_{n}=\left[\begin{array}{ccccccc}
\ddots & & & \vdots & & & \ddots \\
& A_{-2 n} & 0 & 0 & 0 & 0 & \\
\cdots & 0 & A_{-1 n} & 0 & 0 & 0 & \\
& 0 & 0 & A_{0 n} & 0 & 0 & \cdots \\
& 0 & 0 & 0 & 0 & A_{2 n} & \\
\ddots & & & \vdots & & & \ddots
\end{array}\right], \quad \widehat{x}_{n}=\left[\begin{array}{c}
\vdots \\
x_{-2 n} \\
x_{-1 n} \\
x_{0 n} \\
x_{1 n} \\
x_{2 n} \\
\vdots
\end{array}\right]
$$




$$
\begin{aligned}
& \widehat{Z}_{n}=\left[\begin{array}{ccccccc}
\ddots & & & \vdots & & \\
& Z_{0} & Z_{-1} & Z_{-2} & Z_{-3} & Z_{-4} & \\
& Z_{1} & Z_{0} & Z_{-1} & Z_{-2} & Z_{-3} & \\
\cdots & Z_{2} & Z_{1} & Z_{0} & Z_{-1} & Z_{-2} & \cdots \\
& Z_{3} & Z_{2} & Z_{1} & Z_{0} & Z_{-1} & \\
& Z_{4} & Z_{3} & Z_{2} & Z_{1} & Z_{0} & \\
\therefore & & & \vdots & & & \ddots
\end{array}\right], \widehat{T}_{n}=\left[\begin{array}{ccccccc}
\ddots & & & & \\
& T_{0} & T_{-1} & T_{-2} & T_{-3} & T_{-4} & \\
& T_{1} & T_{0} & T_{-1} & T_{-2} & T_{-3} & \\
\cdots & T_{2} & T_{1} & T_{0} & T_{-1} & T_{-2} & \cdots \\
& T_{3} & T_{2} & T_{1} & T_{0} & T_{-1} & \\
& T_{4} & T_{3} & T_{2} & T_{1} & T_{0} & \\
\ddots & & & \vdots & & & \ddots
\end{array}\right], \\
& \widehat{R}_{n}=\left[\begin{array}{ccccccc}
\ddots & & & \vdots & & \\
& R_{0} & R_{-1} & R_{-2} & R_{-3} & R_{-4} & \\
& R_{1} & R_{0} & R_{-1} & R_{-2} & R_{-3} & \\
\cdots & R_{2} & R_{1} & R_{0} & R_{-1} & R_{-2} & \cdots \\
& R_{3} & R_{2} & R_{1} & R_{0} & R_{-1} & \\
& R_{4} & R_{3} & R_{2} & R_{1} & R_{0} & \\
\ddots & & & \vdots & & & \ddots
\end{array}\right], \quad \widehat{\boldsymbol{f}}_{n}=\left[\begin{array}{c}
\vdots \\
\mathbf{0} \\
\mathbf{0} \\
\boldsymbol{f} \\
\mathbf{0} \\
\mathbf{0} \\
\vdots
\end{array}\right]
\end{aligned}
$$

As with the thin-shell model, the matrix is invertible for real material parameter values and the global stiffener matrices are rank-deficient. The unknown coefficients are found by solving

$$
\widehat{\boldsymbol{x}}_{\boldsymbol{n}}=\left[\widehat{\boldsymbol{A}}_{\boldsymbol{n}}+\frac{K_{z}}{a b} \widehat{\boldsymbol{Z}}_{\boldsymbol{n}}+\frac{K_{t}}{a b} \widehat{\boldsymbol{T}}_{\boldsymbol{n}}+\frac{K_{r}}{a b} \widehat{\boldsymbol{R}}_{\boldsymbol{n}}\right]^{-1} \widehat{\boldsymbol{f}}_{\boldsymbol{n}}
$$

The $\hat{\mathbf{x}}_{\mathbf{n}}$ solutions contain the unknown coefficients $A_{m n}-F_{m n}$ which define the displacement coefficients, $W_{m n}, V_{m n}, U_{m n}$, according to $2.4 .6-2.4$.8. Finally, the overall displacements $w(r, \theta$, $z, t), v(r, \theta, z, t)$ and $u(r, \theta, z, t)$ are found using 2.4.2-2.4.4.

\subsection{Excitations}

All excitations considered in this study are harmonic pressure waveforms; in fact, the techniques used to solve the system displacements require a harmonic input. This is not necessarily a restrictive condition as pure waves propagating through a medium, whether solid or fluid, will be spatially and temporally harmonic. Since the main equations of motion are written in terms of stress, the inputs must be given in terms of pressure. Excitations are applied at the outermost shell surface $(r=c)$. There are 2 input types that are applied to the model: a ring load, and an incident plane wave.

\subsubsection{Ring Load Excitation}

The ring load is the simplest input, representing a radial pressure wave that propagates along the cylinder axis, according to

$$
P_{i}(r, \theta, z, t)=P_{0} e^{i k z} e^{-i \omega t}
$$


$P_{0}$ is the constant pressure magnitude, $\omega$ is the excitation frequency, and $k$ is the excitation wavenumber. Ring loads create a radial expansion that travels axially. Note that the ring load is symmetric about the cylinder longitudinal axis, causing no circumferential deformation. While a ring loading may not represent a realistic input, it is very useful for characterization of the system. Figure 2.5.1 shows an example of the cylindrical shell (without stiffeners or damping) response from a ring load with exaggerated magnitude.

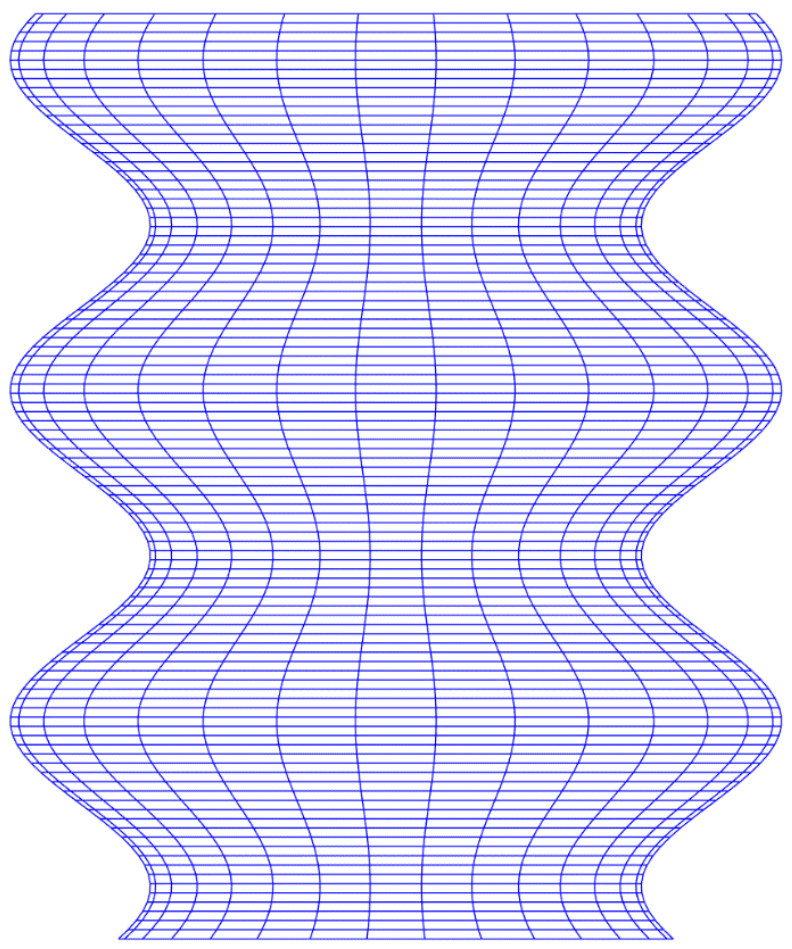

Figure 2.5.1 - Ring load excitation applied to unreinforced cylindrical shell

\subsubsection{Incident Plane Wave (Acoustic Wave)}

Incident plane waves are representative of an incoming oscillatory signal such as sonar, and travel in a distinct direction. They are monochromatic, meaning the excitation frequency is constant. Fluids generally do not support shear wave transmission and the waves considered here are purely dilatational (irrotational) or compressional. Pressure waves used in the model are derived from a plane wave given in Cartesian coordinates as

$$
P_{i}(x, y, z, t)=P_{0} e^{i(\boldsymbol{k} \cdot \boldsymbol{r}-\omega t)}
$$

Unit vector $\mathbf{r}=[\mathrm{x}, \mathrm{y}, \mathrm{z}]^{\top}$ denotes the wave location and vector $\mathbf{k}=\left[\mathrm{k} \cos \phi_{\mathrm{i}}, 0, \mathrm{k} \sin \phi_{\mathrm{i}}\right]^{\top}$ is the excitation wave vector, which denotes the wavenumber and direction of propagation. 


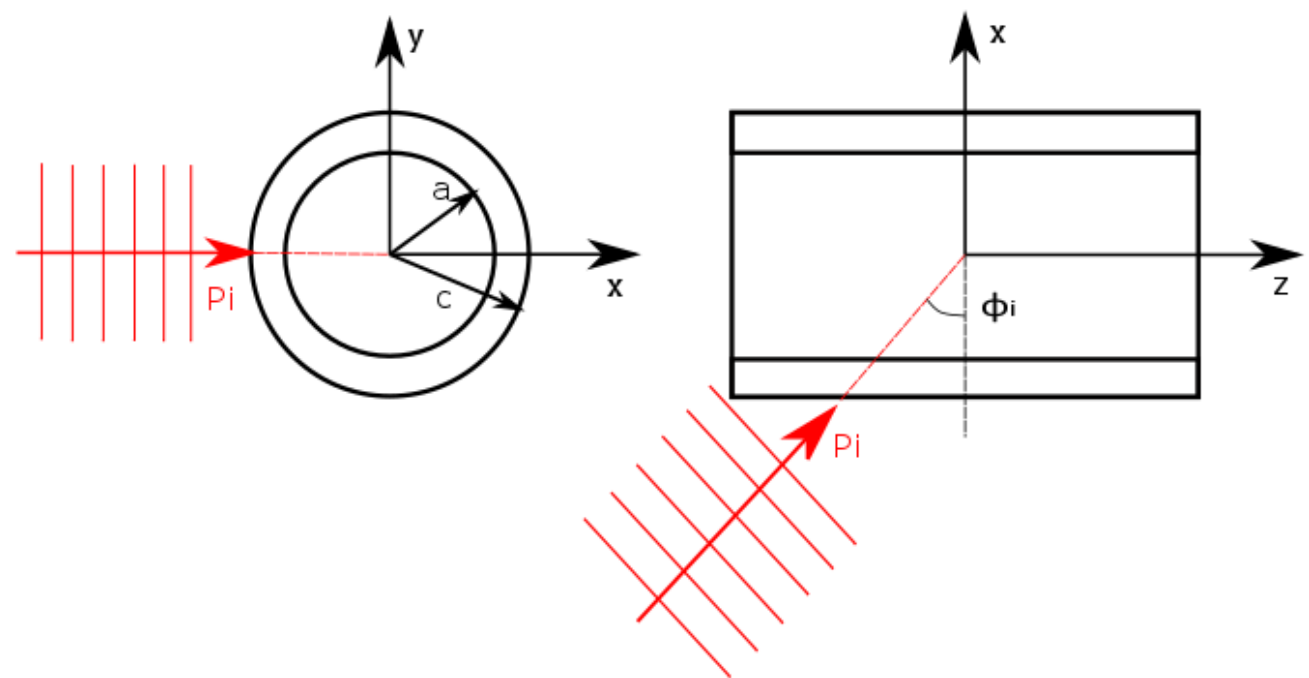

Figure 2.5.2 - Acoustic wave exciting outer cylinder surface, with angle of incidence, $\varphi_{i}$

Plane wave excitations can have an oblique angle of travel relative to the z-axis, shown in Figure 2.5.2 with the angle $\phi_{i}$, but must always point towards the cylinder center-line (cannot be vertically offset, for example). Note that the system is symmetric about cylinder axis, even with stiffeners, so it can always be assumed that the incoming wave is perpendicular with the $y$-axis without loss of generality. For use with the model, the plane wave excitation must be converted into cylindrical coordinates using the relationships

$$
x=r \cos \theta, \quad y=r \sin \theta, \quad z=z
$$

Expanding the original equation results in

$$
P_{i}(x, y, z, t)=P_{0} e^{i(k \cdot r-\omega t)}=P_{0} e^{i k_{x} x} e^{i k_{z} z} e^{-i \omega t}
$$

with $k_{y}=0$ as it is parallel to the $y$-axis, $k_{x}=k_{r}=k \cos \phi_{i}$, and $k_{z}=k \sin \phi_{i}$. The norm of $k$ is related to the excitation frequency and the properties of the fluid

$$
|\boldsymbol{k}|=k=\frac{\omega}{c_{f}}
$$

in which $c_{f}$ is the surrounding fluid wave speed. Unlike the ring load, which simply specifies a wavenumber value, $k$, it makes sense to link the plane wave input to the wave speed of the surrounding medium. Changing the above pressure wave equation into cylindrical coordinates using the relationships above results in

$$
P_{i}(r, \theta, z, t)=P_{0} e^{i k_{z} z} e^{-i \omega t} e^{i k_{r} r \cos \theta}
$$

The last exponential in $r$ and $\theta$ can be expanded using the Jacobi-Anger identity in order to achieve a series of circumferential modes

$$
P_{i}(r, \theta, z, t)=P_{0} e^{i k_{z} z} e^{-i \omega t}\left[J_{0}\left(k_{r} r\right)+2 \sum_{n=1}^{\infty} i^{n} J_{n}\left(k_{r} r\right) \cos (n \theta)\right]
$$


where $J_{n}$ are $n^{\text {th }}$ order Bessel functions of the $1^{\text {st }}$ kind. Finally, the bracketed term can be consolidated by introducing the Neumann factor, $\varepsilon_{\mathrm{n}}$

$$
\varepsilon_{n}= \begin{cases}1, & n=0 \\ 2, & n>0\end{cases}
$$

to result in the condensed form of the plane wave in cylindrical coordinates. The excitation is applied only at the outermost surface of constant radius $(r=c)$

$$
P_{i}(\theta, z, t)=P_{0} e^{i k_{z} z} e^{-i \omega t} \sum_{n=0}^{\infty} \varepsilon_{n} i^{n} J_{n}\left(k_{r} c\right) \cos (n \theta)
$$

Though this form unfortunately involves an infinite series of Bessel functions, it eliminates the last exponential of Equation 2.5.6, and gives the input in terms of circumferential modes which will complement the model solution techniques nicely.

Incident plane wave excitation is not symmetric about the cylindrical axis like the ring load, and can cause circumferential deformations. The resulting deformations can be quite complex even without reinforcement. Figure 2.5.2 shows an example plane wave incident on the left hand side of an unreinforced shell, with exaggerated displacements.

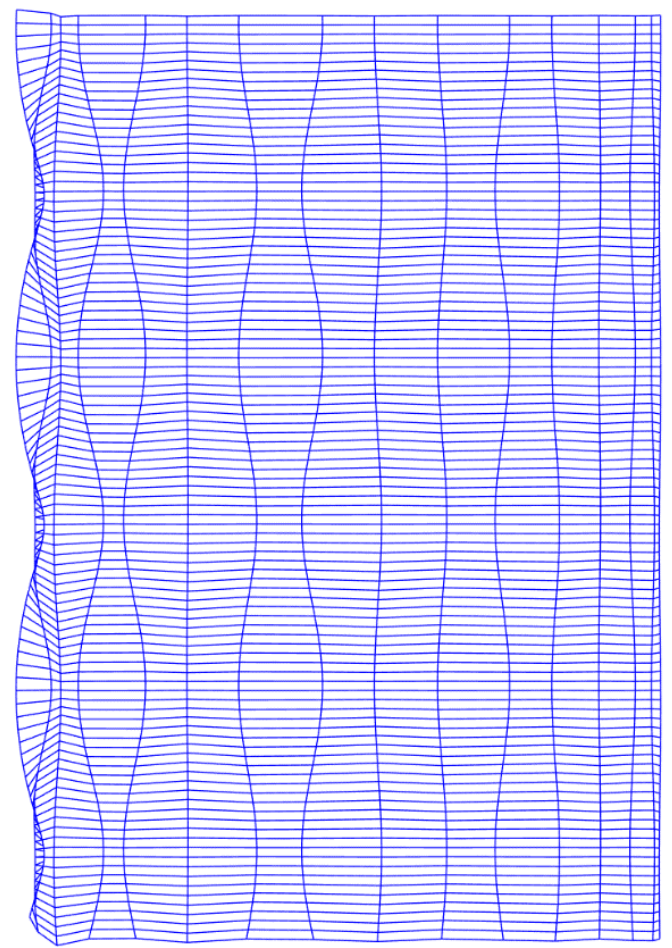

Figure 2.5.2 - Plane Wave excitation applied to the left side of unreinforced cylindrical shell 


\subsubsection{Modifications to System Equations for Plane Wave Input}

Chapter 2.3 and 2.4 fully derived the reference and baseline models excited by a ring load. Recall that $\mathrm{n}>0$ circumferential modes have no effect on the system when excited by a ring loading. For a plane wave load, there are some small but important modifications that must be made to the decoupled and matrix equations. The radial-normal stress, $\tau_{r r}$, at the outer radius ( $r$ $=c$ ) must be rewritten. Equation 2.4.21 is now given as

$$
\begin{aligned}
& \sum_{m=-\infty}^{\infty} \sum_{n=0}^{\infty}\left\{(\lambda+2 \mu) \frac{d W_{m n}(c)}{d r}+\frac{\lambda}{c} W_{m n}(c)+\frac{\lambda n}{c} V_{m n}(c)+\lambda i k_{m} U_{m n}(c)\right\} \cos (n \theta) e^{i k_{m} z} \\
& =P_{0} e^{i k_{z} z} \sum_{n=0}^{\infty} \varepsilon_{n} i^{n} J_{n}\left(k_{r} c\right) \cos (n \theta)
\end{aligned}
$$

with the excitation term shown above now includes an infinite summation of $\mathrm{n}$. The orthogonalization process is performed as before, but $n$-dependence means that the decoupled $\tau_{\mathrm{rr}}$ equation (2.4.36) is also slightly different

$$
\begin{gathered}
{\left[\frac{2 \alpha \mu}{c} J_{n+1}\left(\alpha_{m} c\right)+\left(\frac{2 n(n-1) \mu}{c^{2}}-\alpha_{m}^{2} l \lambda-2 \alpha_{m}^{2} \mu-k_{z m}^{2} \lambda\right) J_{n}\left(\alpha_{m} c\right)\right] A_{m n}} \\
+\left[\frac{2 \alpha \mu}{c} Y_{n+1}\left(\alpha_{m} c\right)+\left(\frac{2 n(n-1) \mu}{c^{2}}-\alpha_{m}^{2} l \lambda-2 \alpha_{m}^{2} \mu-k_{z m}^{2} \lambda\right) Y_{n}\left(\alpha_{m} c\right)\right] B_{m n} \\
+\left[-\frac{2 n \beta_{m} \mu}{c} J_{n+1}\left(\beta_{m} c\right)+\frac{2 n(n-1) \mu}{c^{2}} J_{n}\left(\beta_{m} c\right)\right] C_{m n} \\
+\left[-\frac{2 n \beta_{m} \mu}{c} Y_{n+1}\left(\beta_{m} c\right)+\frac{2 n(n-1) \mu}{c^{2}} Y_{n}\left(\beta_{m} c\right)\right] D_{m n} \\
+\left[-\frac{2 n(n+1) i k_{m} \mu}{c} J_{n+1}\left(\beta_{m} c\right)+2 i k_{m} \beta_{m} \mu J_{n}\left(\beta_{m} c\right)\right] E_{m n} \\
+\left[-\frac{2 n(n+1) i k_{m} \mu}{c} Y_{n+1}\left(\beta_{m} c\right)+2 i k_{m} \beta_{m} \mu Y_{n}\left(\beta_{m} c\right)\right] F_{m n}=P_{0} \varepsilon_{n} i^{n} J_{n}\left(k_{r} c\right) \delta_{m 0}
\end{gathered}
$$

In addition to the new Bessel function, the Kroncker delta only requires $m=0$. Thus the plane wave input is present for all circumferential modes, and these modes now have an effect on the response, unlike the ring load excited system. Grouping the modified equations into a matrix results in the following system

$$
\begin{gathered}
\boldsymbol{A}_{m n} \boldsymbol{x}_{m n}=-\frac{K_{z}}{a L} \sum_{s=-\infty}^{\infty} \boldsymbol{Z}_{m-s} \boldsymbol{x}_{s n}-\frac{K_{t}}{a L} \sum_{s=-\infty}^{\infty} \boldsymbol{T}_{m-s} \boldsymbol{x}_{s n}-\frac{K_{r}}{a L} \sum_{s=-\infty}^{\infty} \boldsymbol{R}_{m-s} \boldsymbol{x}_{s n}+\boldsymbol{f} \delta_{m 0} \\
\boldsymbol{f}=\left[0,0,0, P_{0} \varepsilon_{n} i^{n} J_{n}\left(k_{r} c\right), 0,0\right]^{T}
\end{gathered}
$$

where the $(m, n)$ indexed input vector is multiplied with the new Kronecker delta, $\delta_{m 0}$. All the global equations are unchanged. 


\section{Chapter 3 Base Model Simulation Results}

Before continuing on to develop the more advanced systems (Models $2-5$ from the Introduction), the base model and reference behavior is examined. Unreinforced shell responses are given to illustrate the motion of the shell without stiffeners, and the reference model behavior is shown for both types of excitations. Validation of the new elastic base model with the reference model is an important step, and here the convergence of the model is checked to ensure there is an adequate number of modes present in the simulation. Lastly, the base model is excited with high-frequency inputs and its response is investigated.

\subsection{Shell Displacement Response}

\subsubsection{Model Parameters}

With the baseline reinforced thick-shell model derived in Chapter 2.4, the displacement response can be evaluated for systems with realistic material and geometry. Most applications for reinforced cylinders will require high strength, especially if designing for internal or external fluid loading. Steel (and its alloys) is often a sensible choice for both the shell as well as the rib, and is found in pressure vessels, undersea vehicle hulls, and industrial piping. Table 3.1.1 displays the relevant material properties for the shell and rib. 


\begin{tabular}{|c|c|c|c|}
\hline Property & Symbol & Value & Units \\
\hline Young's Modulus & $\mathrm{E}$ & $210 \times 10^{9}$ & $\mathrm{~N} / \mathrm{m}^{2}$ \\
\hline Poisson's Ratio & $\nu$ & 0.30 & None \\
\hline Structural Damping & $\eta$ & 0.00 & None \\
\hline Density & $\rho$ & 7850 & $\mathrm{~kg} / \mathrm{m}^{3}$ \\
\hline Lamé $1{ }^{\text {st }}$ Parameter & $\lambda$ & $1.212 \times 10^{11}$ & $\mathrm{~N} / \mathrm{m}^{2}$ \\
\hline Shear Modulus & $\mu, \mathrm{G}$ & $8.077 \times 10^{10}$ & $\mathrm{~N} / \mathrm{m}^{2}$ \\
\hline
\end{tabular}

Table 3.1.1 - Material Properties for Shell and Ribs

Some applications may employ a stiffer or softer rib material relative to the shell, depending on the stiffness needs. Often, the primary motivation for choosing a certain rib material is compatibility with the shell, especially in terms of attachment. For welding, a similar alloy metal as the shell is required; for bonding, riveting, or bolted connections, material similarity may not be a concern. The parameters displayed in Table 3.1.1 are used for any shell model, thin or thick. Structural damping $(\eta)$ is implemented as a hysteretic loss component of the elastic modulus, resulting in a complex modulus with the imaginary part dependent upon $\eta$.

$$
E_{\text {total }}=E-E_{\text {loss }} i=E(1-i \eta)
$$

However, for steel, there is very little internal damping and thus the hysteretic element can be ignored. For non-steel materials, the damping factor is important and will be revisited later. Properties such as Young's Modulus and Poisson's Ratio are related to the Lamé Parameters

$$
\lambda=\frac{E_{\text {total }} v}{(1+v)(1-2 v)}, \quad \mu=\frac{E_{\text {total }}}{2(1+v)}
$$

Lambda and mu are found in the derived model equations from prior Chapters. Mu is often referred to as the Shear Modulus (G).

Shell and rib geometry is dependent upon whether the baseline (thick) or reference (thin) model is used. A restrictive assumption of the thin-shell model is that there exits only a single nominal radius with a thickness centered about it. Thus the shell radius for thin-shell systems is the average of the inner and outer radius. Table 3.1.2 displays the relevant dimensions for the shell. 


\begin{tabular}{|c|c|c|c|}
\hline Shell Property & Symbol & Value & Units \\
\hline Inner Radius & $\mathrm{a}$ & 4.500 & $\mathrm{~m}$ \\
\hline Thickness & $\mathrm{h}$ & 0.0254 & $\mathrm{~m}$ \\
\hline Outer Radius & $\mathrm{c}$ & 4.5254 & $\mathrm{~m}$ \\
\hline Rib Spacing & $\mathrm{L}$ & 0.8 & $\mathrm{~m}$ \\
\hline
\end{tabular}

Table 3.1.2 - Dimensional Properties for Shell

Rib dimensions are dependent upon the cross-section. For this study, rectangular cross-sections are used, as described in Figure 2.2.3 of Chapter 2.2. Table 3.1.3 displays the pertinent dimensions for the stiffening ribs.

\begin{tabular}{|c|c|c|c|}
\hline Rib Property & Symbol & Value & Units \\
\hline Width & $\mathrm{b}$ & 0.0254 & $\mathrm{~m}$ \\
\hline Height & $\mathrm{h}_{\mathrm{r}}$ & 0.1016 & $\mathrm{~m}$ \\
\hline Radius & $\mathrm{ar}$ & 4.4374 & $\mathrm{~m}$ \\
\hline Area (Cross-section) & $\mathrm{A}$ & 0.00258 & $\mathrm{~m}^{2}$ \\
\hline Area Moment of Inertia & $\mathrm{I}$ & $2.083 \times 10^{-6}$ & $\mathrm{~m}^{4}$ \\
\hline Polar Moment of Inertia & $\mathrm{J}$ & $4.391 \times 10^{-7}$ & $\mathrm{~m}^{4}$ \\
\hline
\end{tabular}

Table 3.1.3 - Dimensional Properties for Ribs

The last 4 properties of Table 3.1.3 are computed from the rib width and height.

$$
a_{r}=a-\frac{h}{2}-\frac{h_{r}}{2}, \quad A=b h_{r}, \quad I=\frac{1}{12} b h_{r}^{3}, \quad J=0.281 b^{3} h_{r}
$$

\subsubsection{Simulation Settings}

After establishing the model parameters for the chosen material and geometry, the settings for the simulation must be determined. Recall the displacement field equations from the base model (Equations 2.4.2 - 2.4.4)

$$
\begin{aligned}
& u(r, \theta, z, t)=\sum_{m=-\infty}^{\infty} \sum_{n=0}^{\infty} U_{m n}(r) \cos (n \theta) e^{i k_{m} z} e^{-i \omega t} \\
& v(r, \theta, z, t)=\sum_{m=-\infty}^{\infty} \sum_{n=0}^{\infty} V_{m n}(r) \sin (n \theta) e^{i k_{m} z} e^{-i \omega t} \\
& w(r, \theta, z, t)=\sum_{m=-\infty}^{\infty} \sum_{n=0}^{\infty} W_{m n}(r) \cos (n \theta) e^{i k_{m} z} e^{-i \omega t}
\end{aligned}
$$


First, the temporal frequency, $\omega$, for the input must be chosen; see the Excitations section of Chapter 2.5. Since the system is linear, the displacement response has the same harmonic frequency as the excitation wave; $\omega$ in 3.1.4-3.1.6 is the same as the input. Unfortunately, the 3 displacement equations involve infinite summations, which must be approximated to execute a simulation. By using a finite number of terms that are large enough to represent all relevant motion, the real system can be simulated.

\begin{tabular}{|c|c|c|}
\hline Setting & Symbol & Units \\
\hline Input Frequency & $\omega$ & $\mathrm{rad} / \mathrm{s}$ \\
\hline $\begin{array}{c}\text { Number of } \\
\text { Circumferential Modes }\end{array}$ & $\mathrm{n}_{\mathrm{pts}}$ & None (Integer) \\
\hline Number of Axial Modes & $\mathrm{m}_{\mathrm{pts}}$ & None (Integer) \\
\hline
\end{tabular}

Table 3.1.4 - Simulation settings

The settings shown in Table 3.1.4 will be varied for different simulations. Integer circumferential and axial modes are set on the intervals

$$
n: 0,1,2,3, \ldots, n_{p t s} \quad m:-\frac{m_{p t s}}{2}, \ldots,-2,-1,0,1,2, \ldots, \frac{m_{p t s}}{2}
$$

to account for the limits on the respective summations. For odd values of $m_{\text {pts, }}$ floor or ceiling functions ensure that $m_{\text {pts }} / 2$ is an integer. 


\subsubsection{Unreinforced Model Results}

This is study is focused on the spatial displacement responses, such that the temporal component is not considered. However, the range of excitation frequencies has an important effect on the spatial response, particularly if the input wavenumber and frequency are coupled $\left(k=\omega / c_{f}\right)$. Both ring loading and plane-wave loadings will be discussed. Parameters presented in Tables 3.1.1 - 3.1.3 are constant during this study, but each model will have additional parameters that must be set (fluid, beam, etc) in addition to the simulation settings displayed in Table 3.1.4.

The unreinforced model has been explored by many authors including Gazis and Graf, and the responses are included in this study to better show the effect of the stiffeners. First the ring loading is examined. Since there are no stiffeners, only the $\mathrm{m}=0$ mode is needed; since the input is a ring load, only the $n=0$ circumferential mode affects the model.

\begin{tabular}{|c|c|c|c|}
\hline Setting & Symbol & Value & Units \\
\hline Input Frequency & $\mathrm{f}$ & 10 to 10000 & $\mathrm{~Hz}$ \\
\hline Input Wavenumber & $\mathrm{k}$ & 0 or $2 \pi \mathrm{f} / \mathrm{cf}$ & $1 / \mathrm{m}$ \\
\hline Thickness & $\mathrm{h}$ & 0.0254 & $\mathrm{~m}$ \\
\hline $\begin{array}{c}\text { Number of } \\
\text { Circumferential Modes }\end{array}$ & $\mathrm{n}_{\mathrm{pts}}$ & 1 or 101 & None \\
\hline Number of Axial Modes & $\mathrm{m}_{\mathrm{pts}}$ & 1 & None \\
\hline
\end{tabular}

Table 3.1.5 - Unreinforced base model (elastic) settings

If the excitation wavenumber $k=0$, then the radial response is constant along the cylinder length. Figure 3.1.1 and 3.1.2 display the unreinforced response of the shell for this input. 

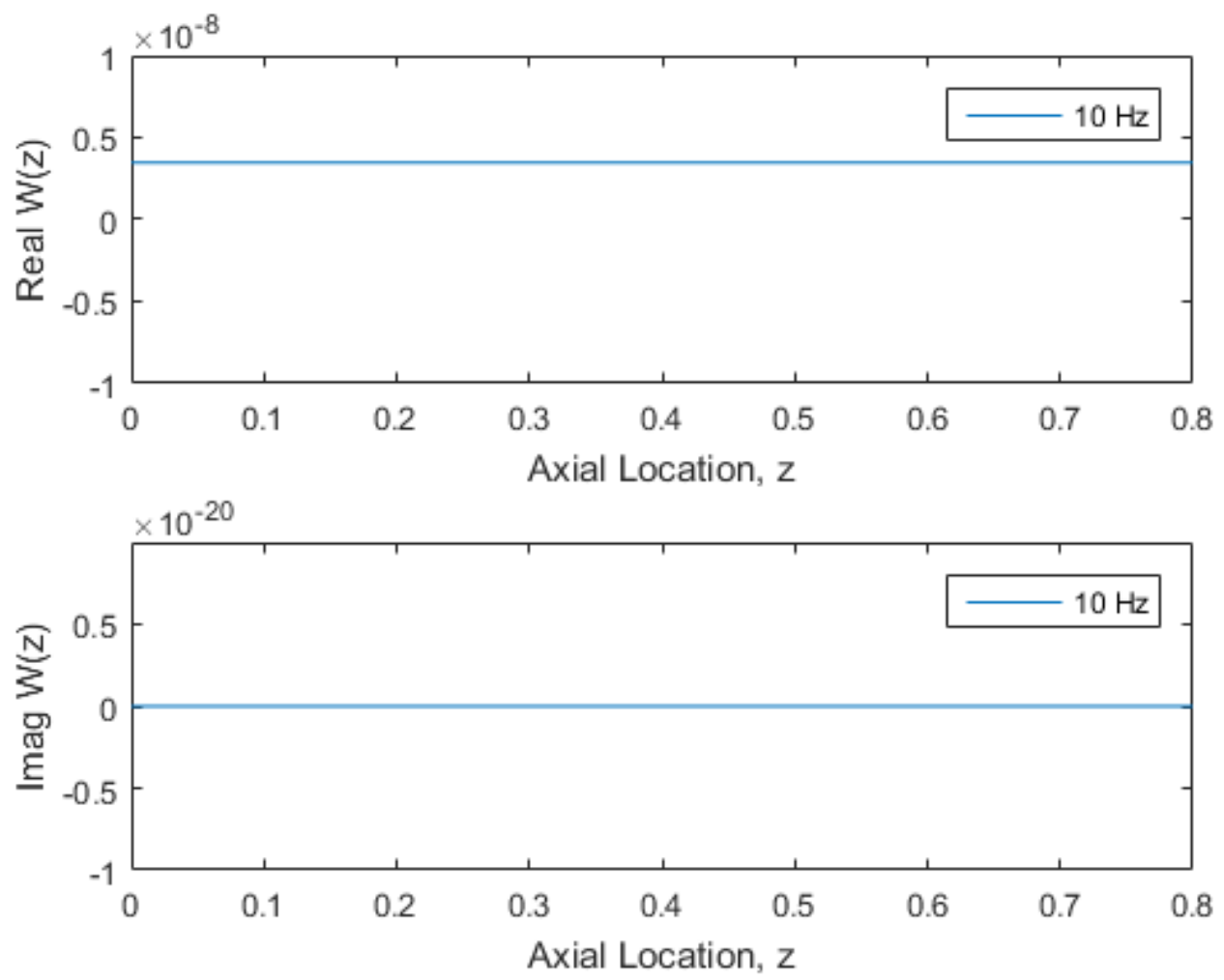

Figure 3.1.1 - Unreinforced radial response for $\operatorname{ring} \operatorname{load}(\mathrm{k}=0, \mathrm{f}=10 \mathrm{hz})$
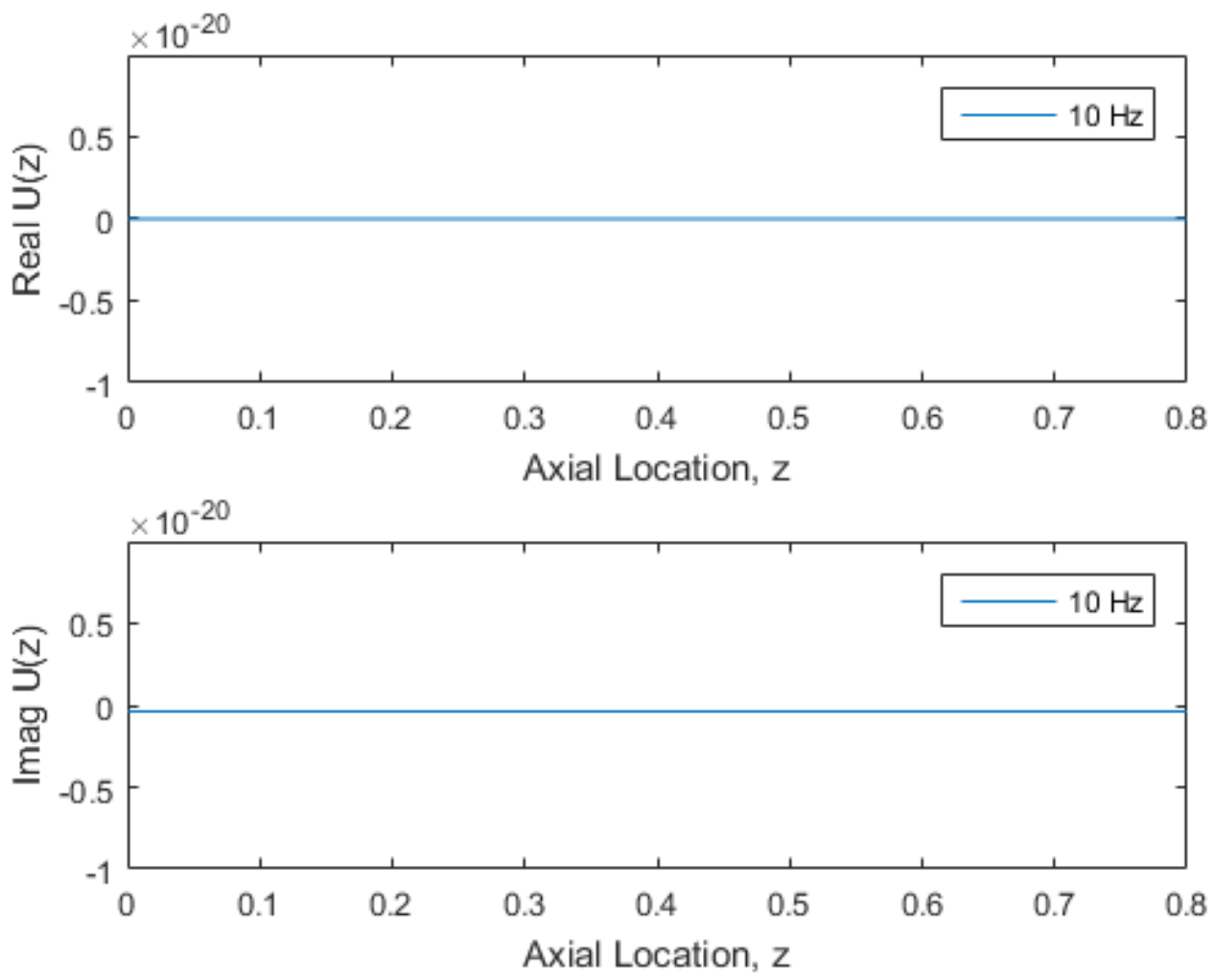

Figure 3.1.2 - Unreinforced axial response for $\operatorname{ring} \operatorname{load}(\mathrm{k}=0, \mathrm{f}=10 \mathrm{hz})$ 
There is no axial displacement in the shell, because the ring load only applies a radial pressure; the displacement is constant along the cylinder length, as expected. Excitation frequency can be swept from $10 \mathrm{~Hz}$ to $10 \mathrm{kHz}$ to determine any notable resonances.

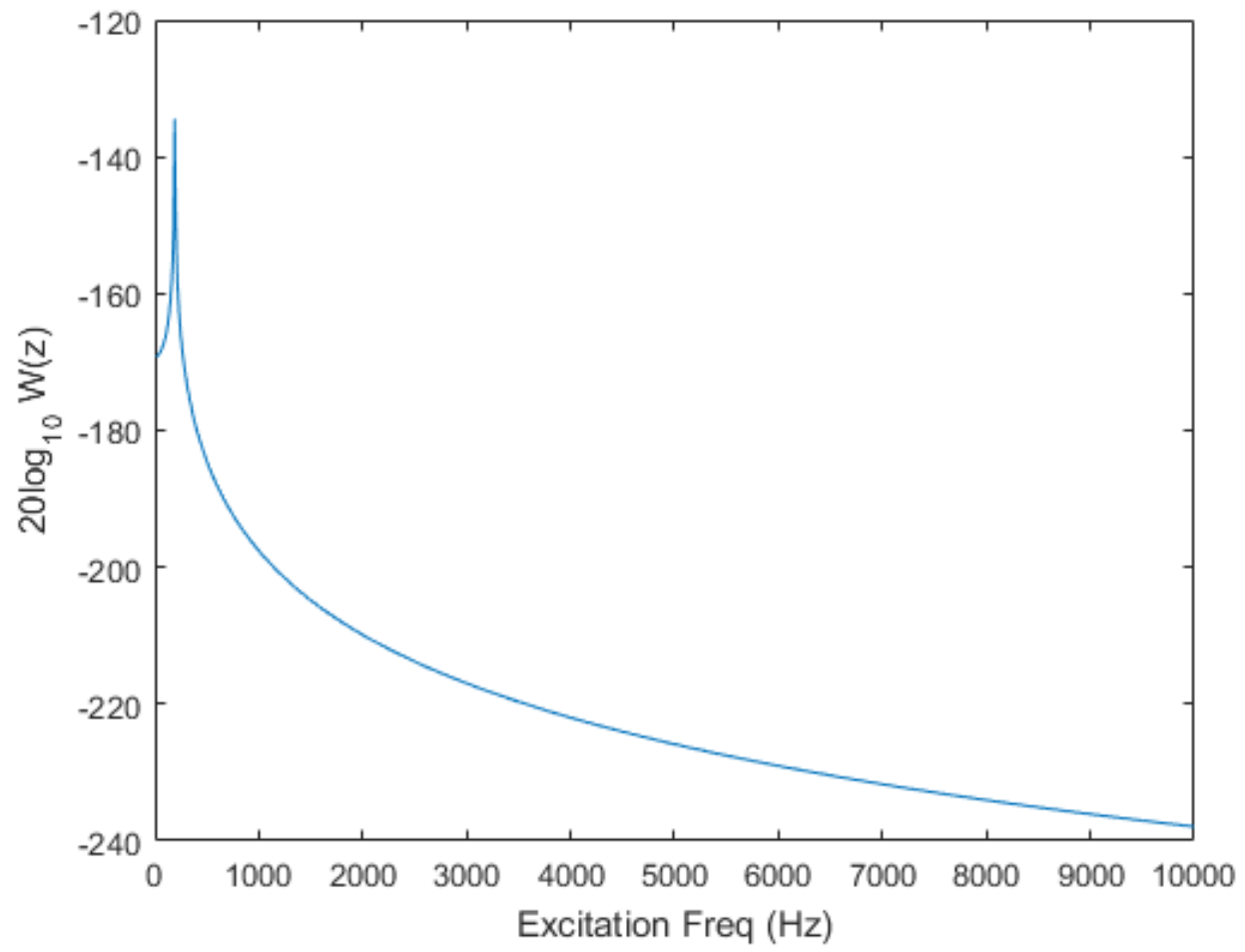

Figure 3.1.3 - Unreinforced radial frequency response $(\mathrm{z}=0)$ for ring load $(\mathrm{k}=0)$

The $z$-constant ring load causes only a single radial resonance at $190 \mathrm{~Hz}$. When $k=\omega / c_{f}$, where $c_{f}$ is a nominal fluid wave speed $(\mathrm{m} / \mathrm{s})$, the ring load excitation is no longer constant along the cylinder length, and the shell displacements now vary axially. 

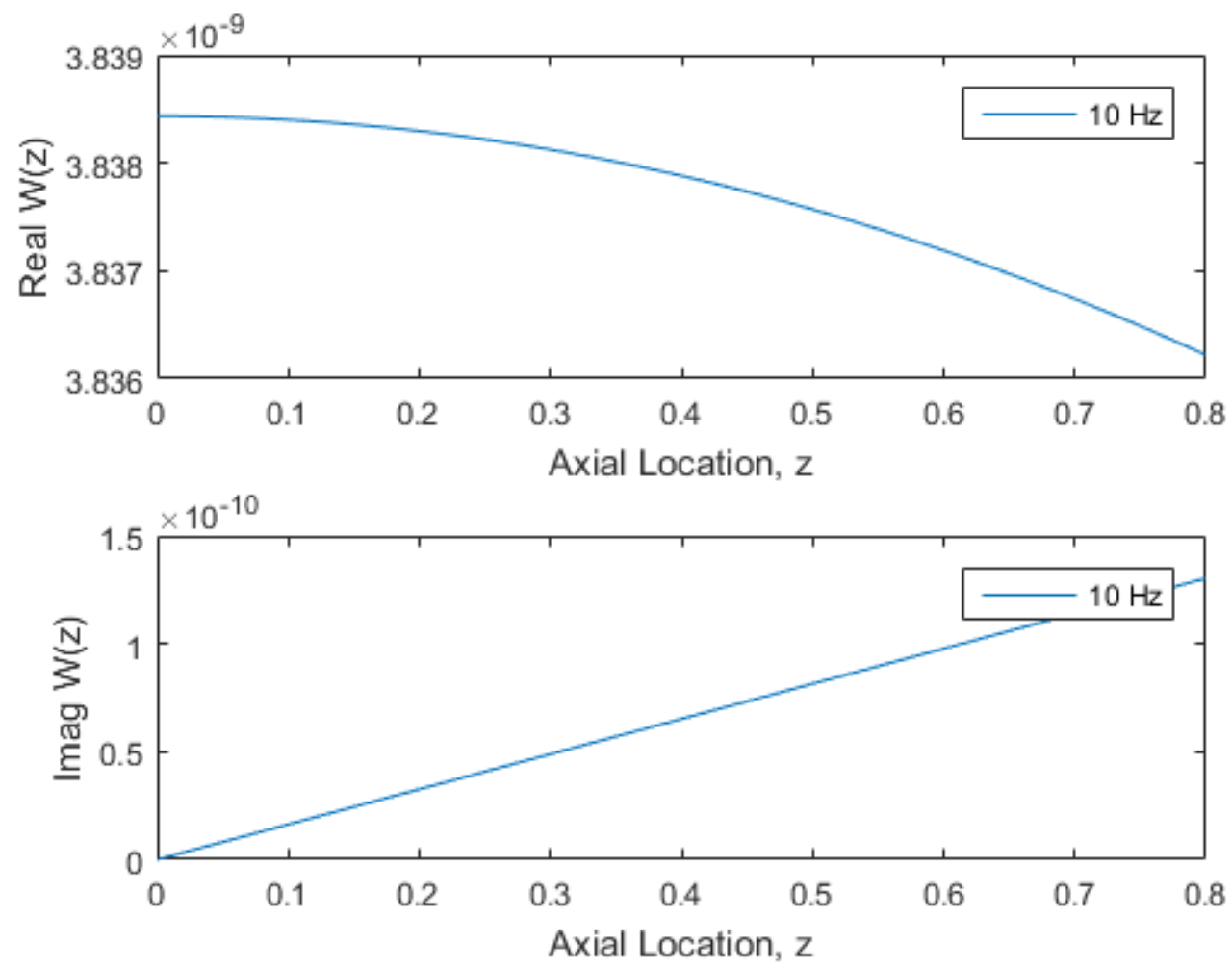

Figure 3.1.4 - Unreinforced radial response for $\operatorname{ring} \operatorname{load}(\mathrm{k}=\omega / \mathrm{c} f, \mathrm{f}=10 \mathrm{hz})$
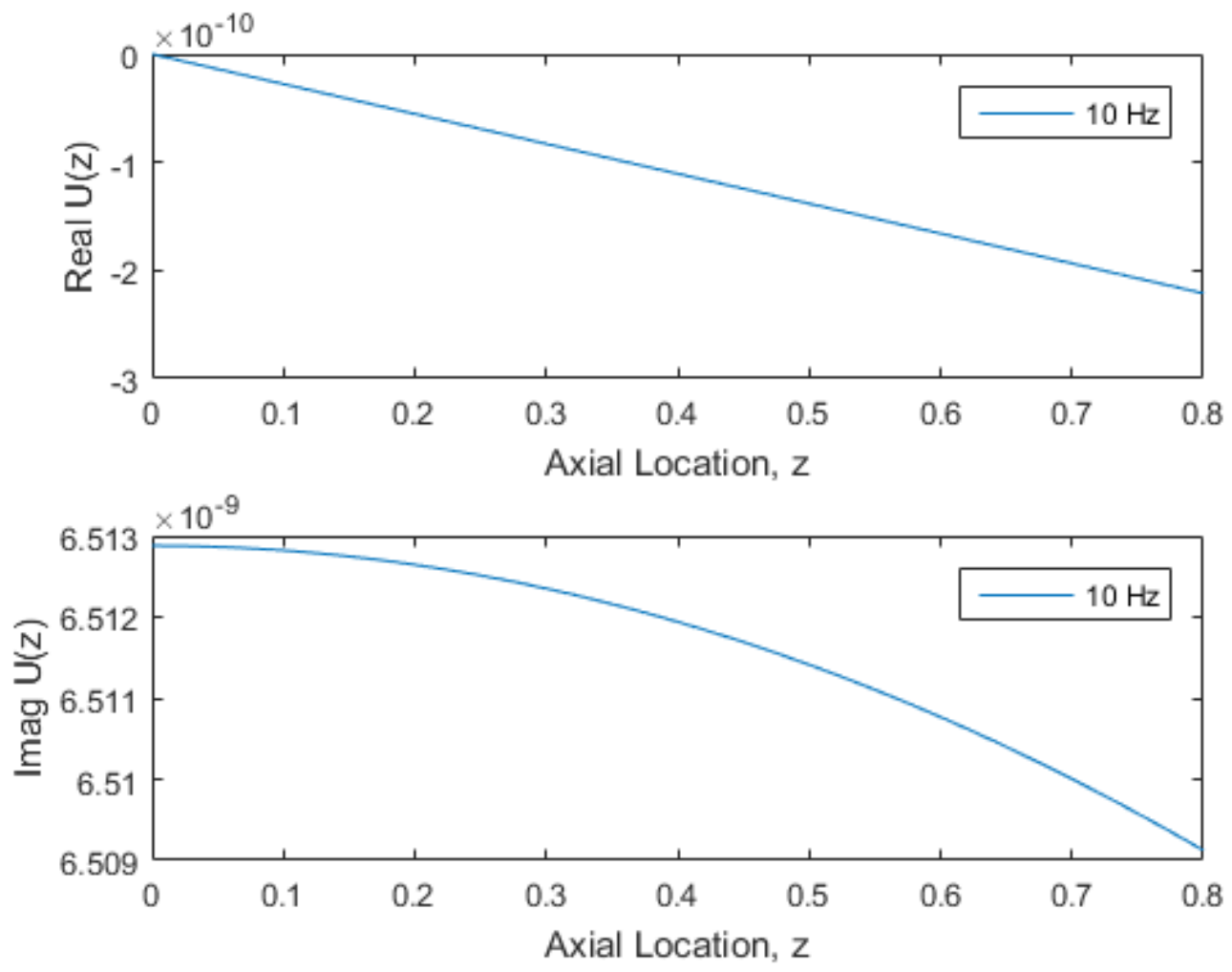

Figure 3.1.5 - Unreinforced axial response for $\operatorname{ring} \operatorname{load}\left(k=\omega / \mathrm{c}_{\mathrm{f}}, \mathrm{f}=10 \mathrm{hz}\right)$ 
Increasing the excitation frequency to $5000 \mathrm{~Hz}$ shows the sinusoidal nature of the shell motion, seen in Figure 3.1.6 and 3.1.7.
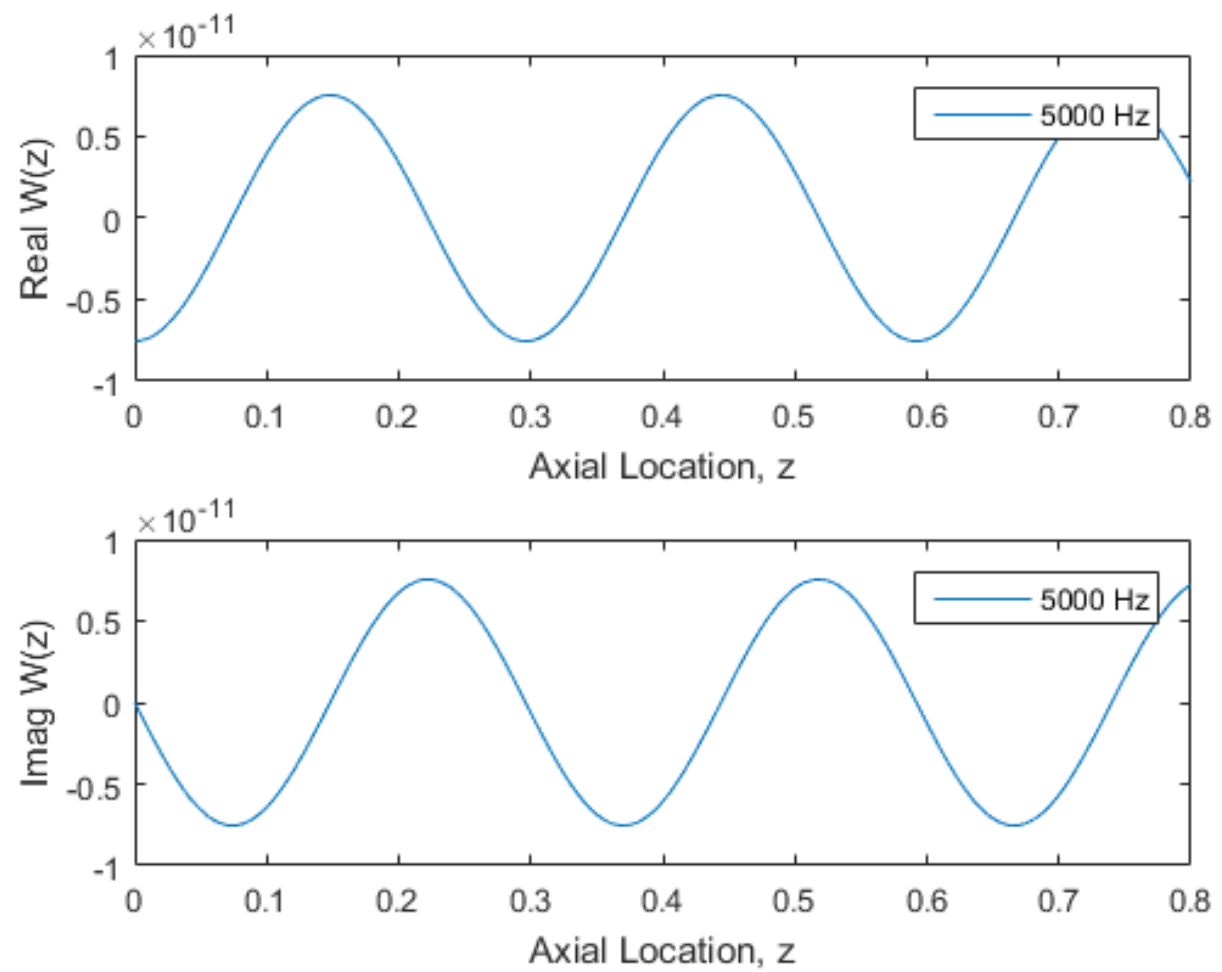

Figure 3.1.6 - Unreinforced radial response for $\operatorname{ring}$ load $(\mathrm{k}=\omega / \mathrm{cf}, \mathrm{f}=5000 \mathrm{hz})$
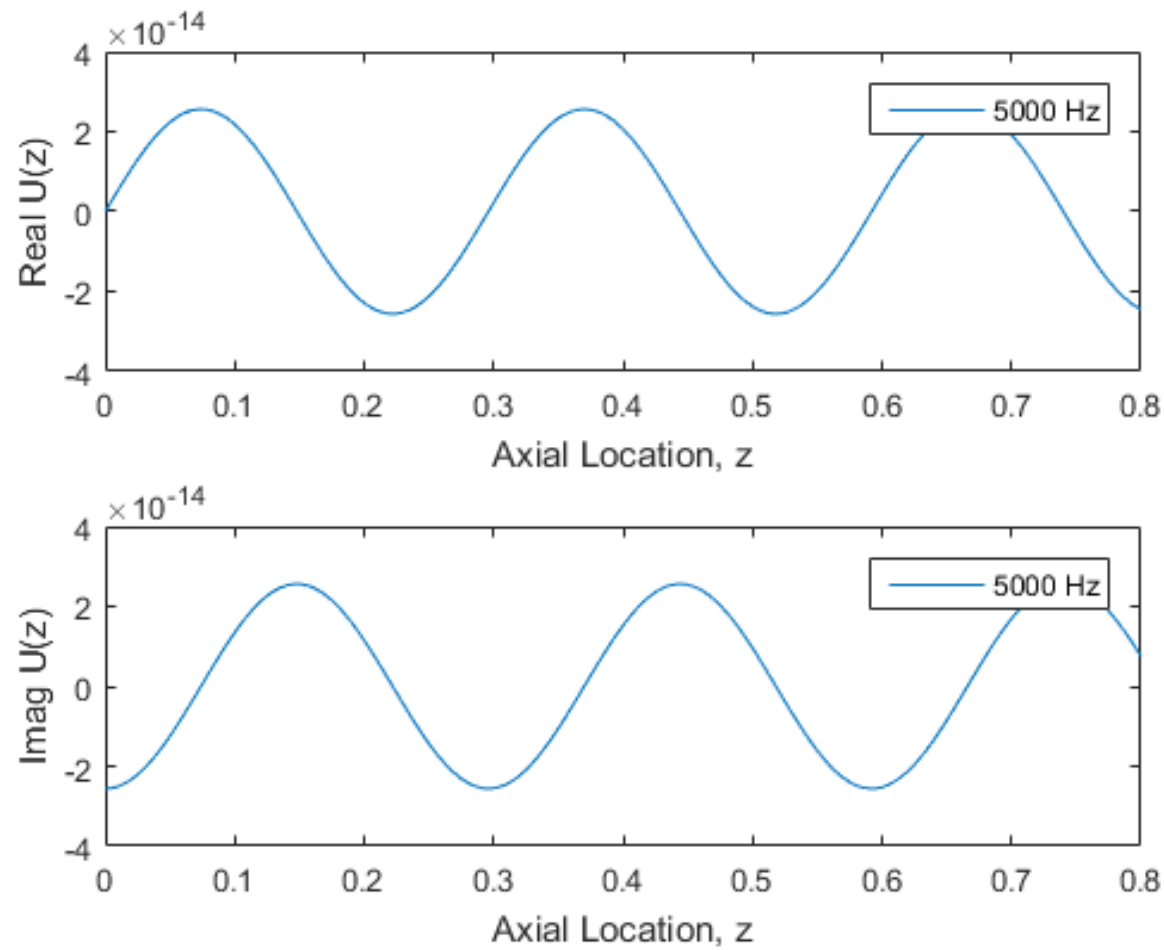

Figure 3.1.7 - Unreinforced axial response for $\operatorname{ring}$ load $\left(\mathrm{k}=\omega / \mathrm{c}_{\mathrm{f}}, \mathrm{f}=5000 \mathrm{hz}\right)$ 
Figures 3.1.4 to 3.1.7 have a constant magnitude, but are broken down into real and imaginary components. Note that there is now an axial response as well as a radial response to the ring loading. If the excitation frequency $\omega=2 \pi c_{f} / L=3700 \pi$ then there will be one sinusoidal period along the $z$ axis. As before, the input is swept from 10 to $10000 \mathrm{~Hz}$, but there is both an axial and radial response.

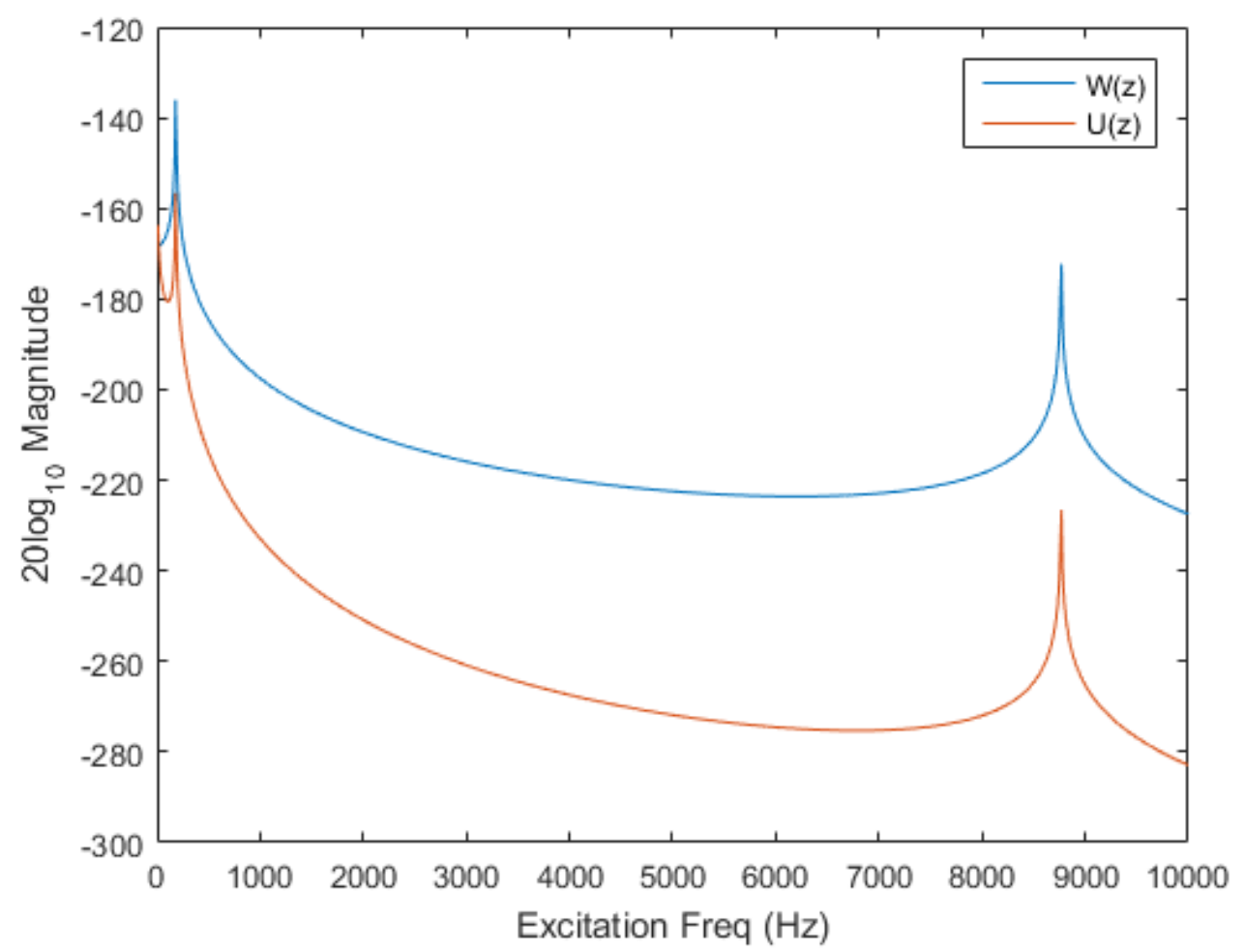

Figure 3.1.8 - Unreinforced radial/axial frequency response $(\mathrm{z}=0)$ for $\operatorname{ring}$ load $(\mathrm{k}=\omega / \mathrm{cf})$

There are 2 resonances of the system now, the radial resonance at $180 \mathrm{~Hz}$ and a longitudinal resonance at $8770 \mathrm{~Hz}$.

Next the plane-wave excitation is applied to the system, at an oblique angle $\phi_{i}=\pi / 12$, which is 15 degrees from a broadside impact (see Chapter 2.5). Plane-wave excitation is not circumferentially symmetric, and thus non-zero circumferential modes $(n>0)$ are required; $n_{p t s}$ $=101$ is chosen to ensure that any important modes are not being ignored. Figure 3.1.8 displays the total cylinder response for a $10 \mathrm{hz}$ plane-wave excitation. 

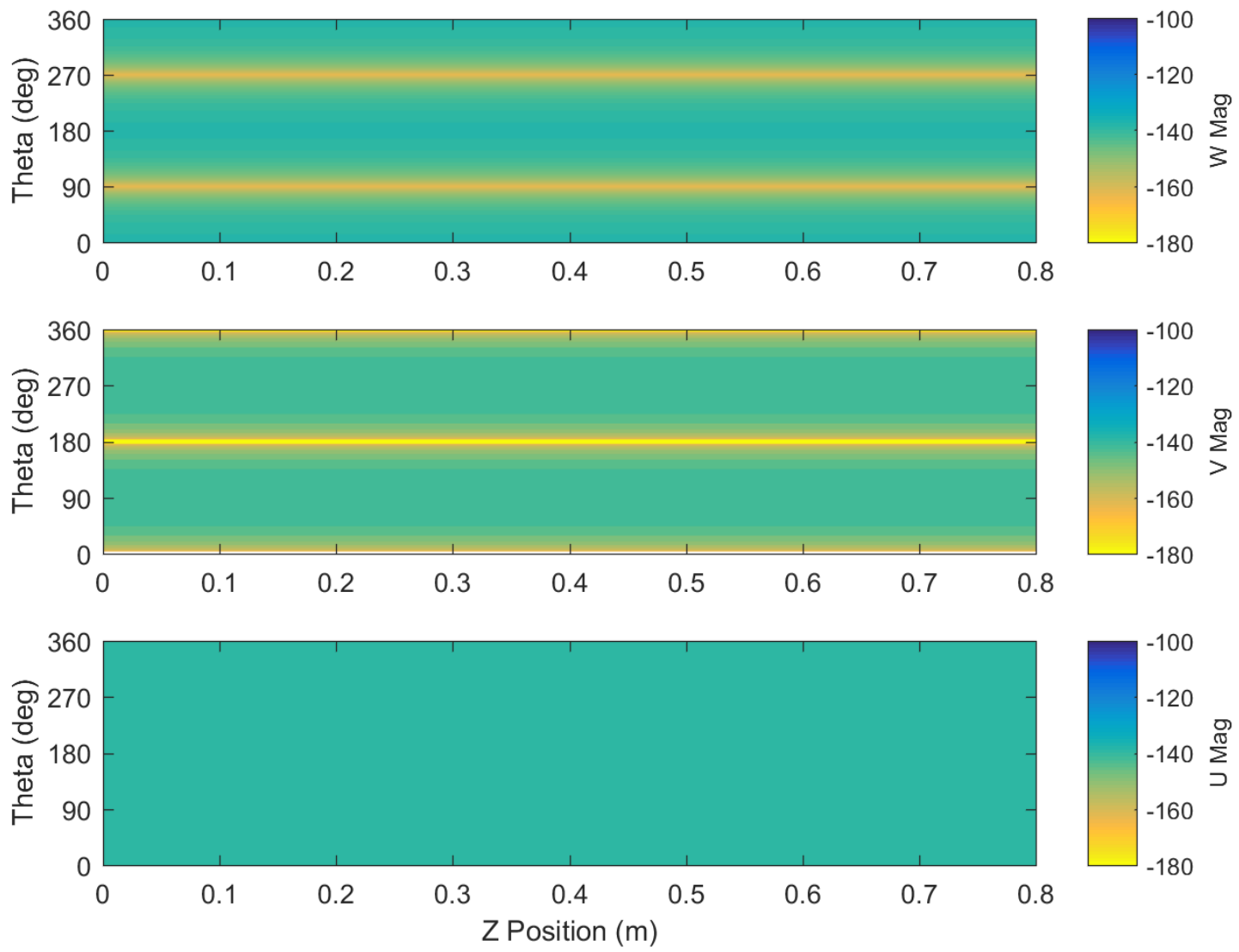

Figure 3.1.9 - Unreinforced cylinder radial (top), tangential (mid), and axial (bottom) displacement response for plane-wave: $10 \mathrm{~Hz}, \varphi_{\mathrm{i}}=\pi / 12, \mathrm{n}_{\mathrm{pts}}=101$

Figure 3.1.9 is essentially an unwrapped cylinder, with the vertical axis designating the angular position around the circumference and the horizontal axis designating the axial position along the cylinder length. Contour color designates the magnitude of the displacement in absolute value (log scale). The plane wave input is not symmetric about the cylinder axis and impacts at $\theta$ $=0$. At this angular position, the radial magnitude is largest, and the tangential is a minimum (scales are equal); the tangential deformation is inverted relative to the radial deformation, but there is little angular dependence for the axial displacement. This shows behavior dominated by a low order circumferential mode: the middle plot shows a dilatational circumferential mode and the upper plot shows a circumferential shear mode. For dilatational waves, the amplitude is parallel to the direction of travel; for shear waves, the amplitude is perpendicular to the direction of travel. Shear waves are denoted by the direction of wave propagation and the direction of amplitude is assumed by context. At $5000 \mathrm{~Hz}$, the shape is similar but the magnitudes have decreased significantly, requiring a change in scale. 

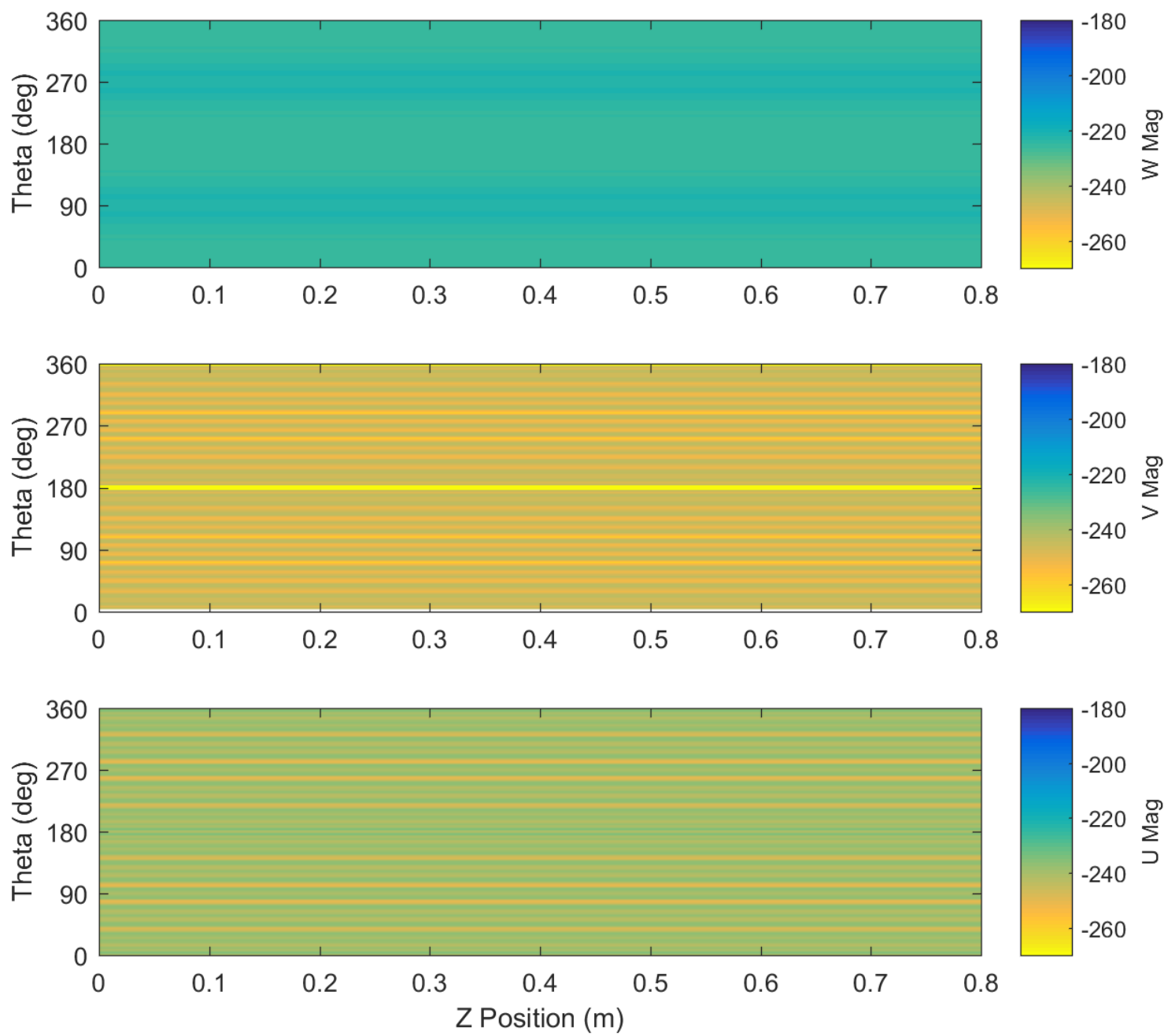

Figure 3.1.10 - Unreinforced cylinder radial (top), tangential (mid), and axial (bottom) displacement response for plane-wave: $5000 \mathrm{~Hz}, \varphi_{\mathrm{i}}=\pi / 12, \mathrm{n}_{\mathrm{pts}}=101$

At $5000 \mathrm{~Hz}$, there are numerous bands indicating that the motion is dominated by a higher-order circumferential mode: the lower plot indicates a circumferential shear mode is present. Unfortunately, sweeping excitation frequency with an applied plane-wave does not show the distinct resonances of the ring loading. 


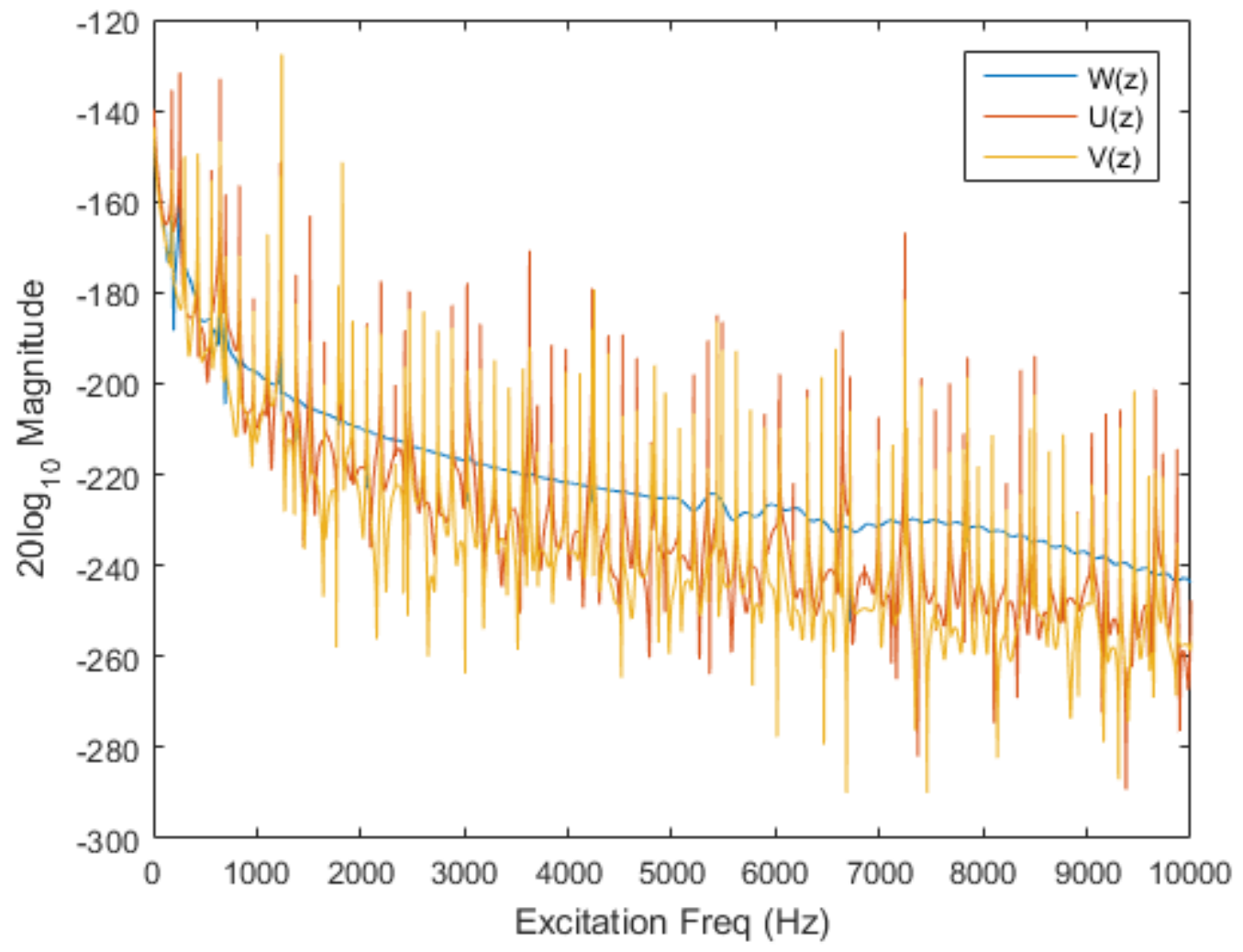

Figure 3.1.11 - Unreinforced radial/axial/tangential frequency response $(\mathrm{z}=0, \theta=36 \mathrm{deg})$ for plane-wave loading

The frequency response of the shell at position $z=0$ and $\theta=36 \mathrm{deg}$ shows harmonics of a $137 \mathrm{~Hz}$ fundamental frequency. Ring-loading resonances of $180 \mathrm{~Hz}$ and $8770 \mathrm{~Hz}$ are present, but are not distinct from the other harmonics. All three displacements have matching resonances. Even for unreinforced systems, the frequency response is not especially helpful.

\subsubsection{Thin-Shell Model Results}

The Donnell-based model derived in Chapter 2.3 provides the validation for the newly developed elastic base model (Chapter 2.4). Hull [19] used FEA to support the results from the thin-shell system and achieved excellent agreement between the two methods. FEA is outside the scope of this study, but the elastic model can be configured to match the thin-shell system. Thus, the thin-shell results will be presented first using the data described above. The number of modes ( $m_{\text {pts }}$ and $n_{\text {pts }}$ ) simulated is very limiting because the computational cost can become substantial, especially when evaluating Bessel functions.

By design, the ring load is symmetric about the cylinder axis, such that there is no tangential displacement and circumferential modes have no effect on the forced response (see Equation 2.3.28). Thus the radial and axial displacement is constant around the shell circumference, so it is only necessary to observe the response at a single point on the circumference; $\theta=0$ is chosen for all ring load responses. Table 3.1.6 shows the parameters chosen for the base thin-shell model with delta connectors and linear spring stiffeners. 


\begin{tabular}{|c|c|c|c|}
\hline Setting & Symbol & Value & Units \\
\hline Input Frequency & $\mathrm{f}$ & 10 to 5000 & $\mathrm{~Hz}$ \\
\hline Input Wavenumber & $\mathrm{k}$ & 0 or $2 \pi \mathrm{ff} / \mathrm{cf}$ & $1 / \mathrm{m}$ \\
\hline Thickness & $\mathrm{h}$ & 0.001 & $\mathrm{~m}$ \\
\hline $\begin{array}{c}\text { Number of } \\
\text { Circumferential Modes }\end{array}$ & $\mathrm{n}_{\mathrm{pts}}$ & 1 or 21 & None \\
\hline Number of Axial Modes & $\mathrm{m}_{\mathrm{pts}}$ & 201 & None \\
\hline Rib Stiffness Radial & $\mathrm{Kr}_{\mathrm{r}}$ & $1 \times 10^{10}$ & $\mathrm{~N} / \mathrm{m}$ \\
\hline Rib Stiffness Tangential & $\mathrm{K}_{\mathrm{t}}$ & $1 \times 10^{10}$ & $\mathrm{~N} / \mathrm{m}$ \\
\hline Rib Stiffness Axial & $\mathrm{K}_{\mathrm{z}}$ & $1 \times 10^{10}$ & $\mathrm{~N} / \mathrm{m}$ \\
\hline
\end{tabular}

Table 3.1.6 - Thin-shell base model settings

Instead of displaying real and imaginary components, which begin to become confusing when the model is reinforced, the magnitude in absolute value (log scale) will be plotted. Displacement magnitude is more important than the direction of displacement especially at higher frequencies. Figure 3.1.12 and 3.1.13 show the radial and axial displacements of the thin-shell model for a $10 \mathrm{~Hz}, 1000 \mathrm{~Hz}$, and $5000 \mathrm{~Hz}$ ring load with $\mathrm{k}=0$ wavenumber.

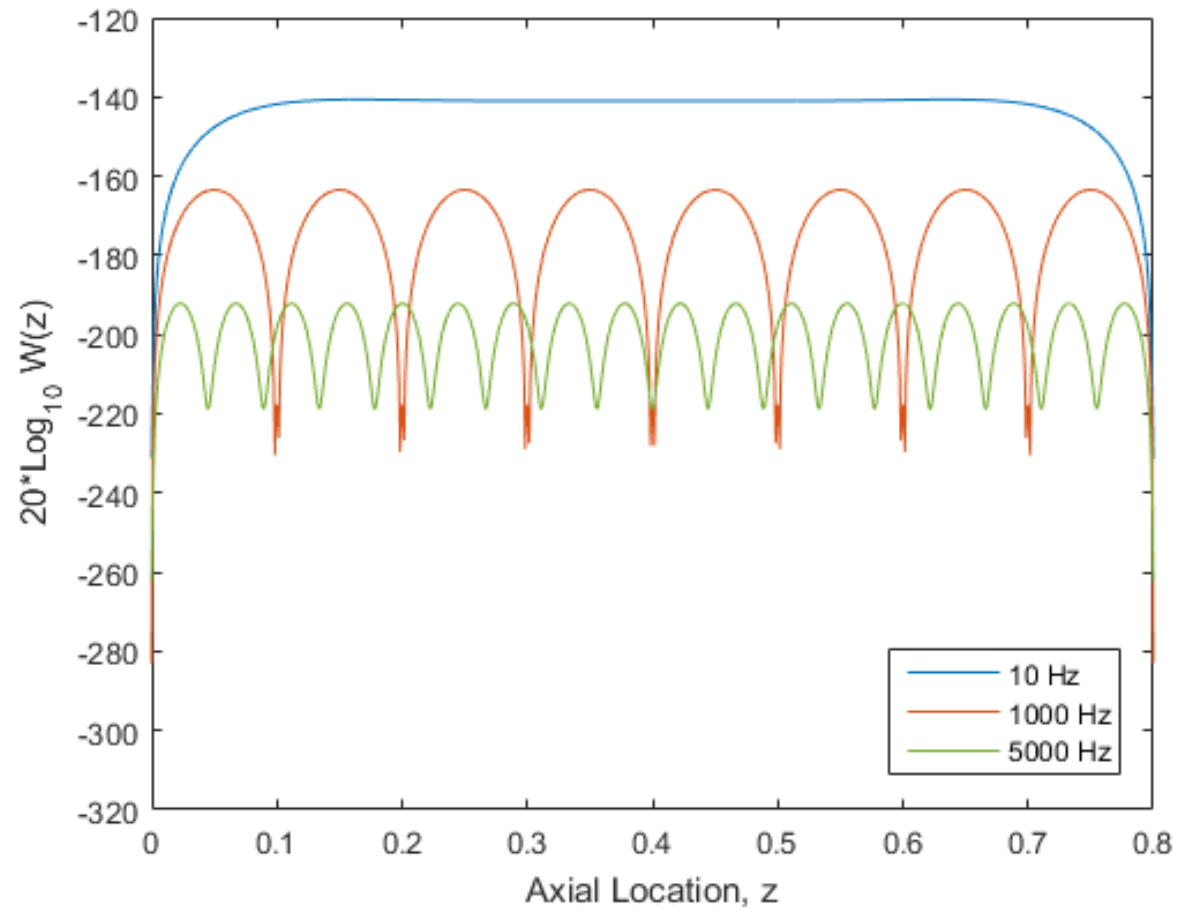

Figure 3.1.12 - Thin-shell radial response for $\operatorname{ring} \operatorname{load}(\mathrm{k}=0, \mathrm{f}=10,1000,5000 \mathrm{~Hz})$ 


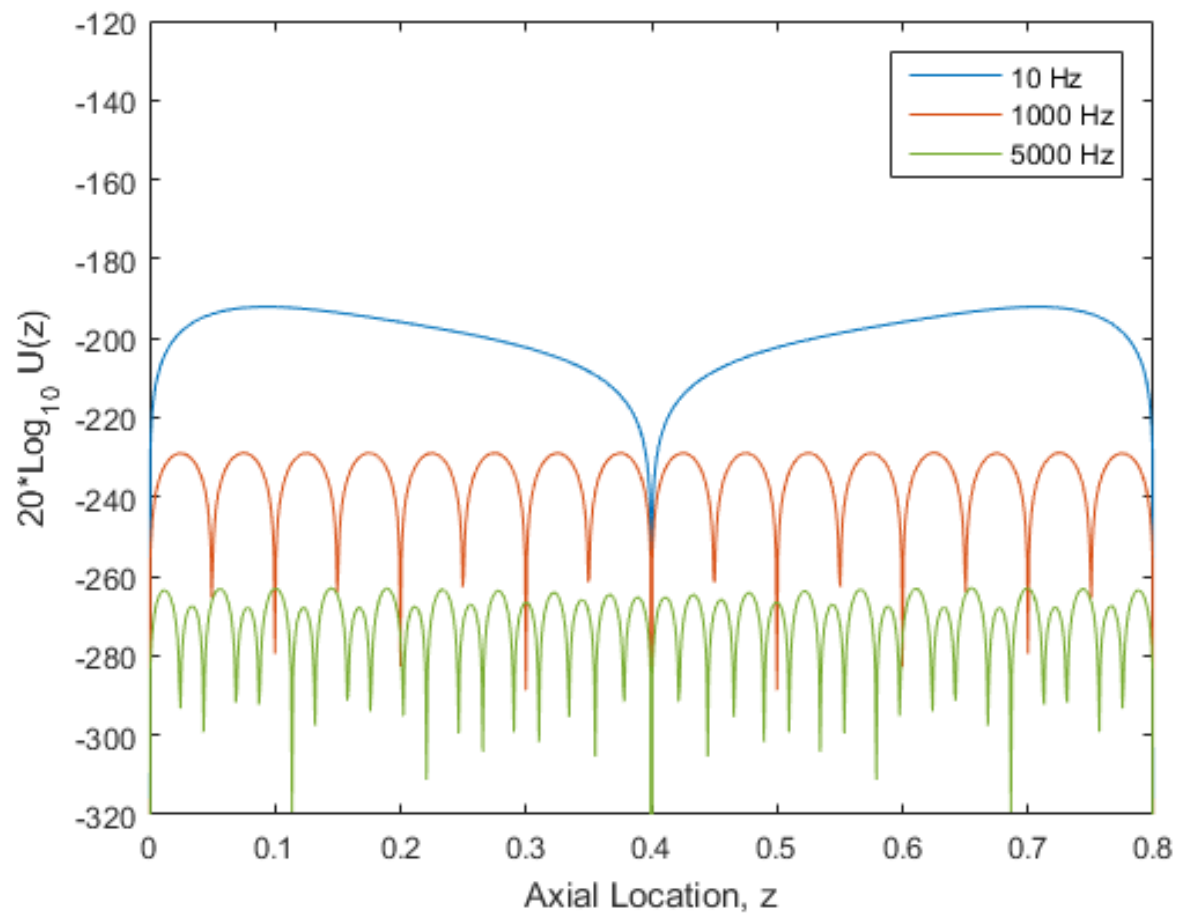

Figure 3.1.13 - Thin-shell axial response for $\operatorname{ring} \operatorname{load}(\mathrm{k}=0, \mathrm{f}=10,1000,5000 \mathrm{~Hz})$

Unlike the unreinforced model, the thin-shell model with periodic stiffeners has a significant axial response even though the input is purely radial with no axial variation. Both the thin-shell and thick-shell equations of motion are coupled, so if the system becomes non-uniform (due to the ribs) then the response will be coupled. Responses are sinusoidal and symmetric about the midpoint of the cylinder length $(z=0.4)$; magnitudes are far lower than the low frequency case, as expected. For the $k=\omega / c_{f}$ wavenumber input, the responses become more complicated. 


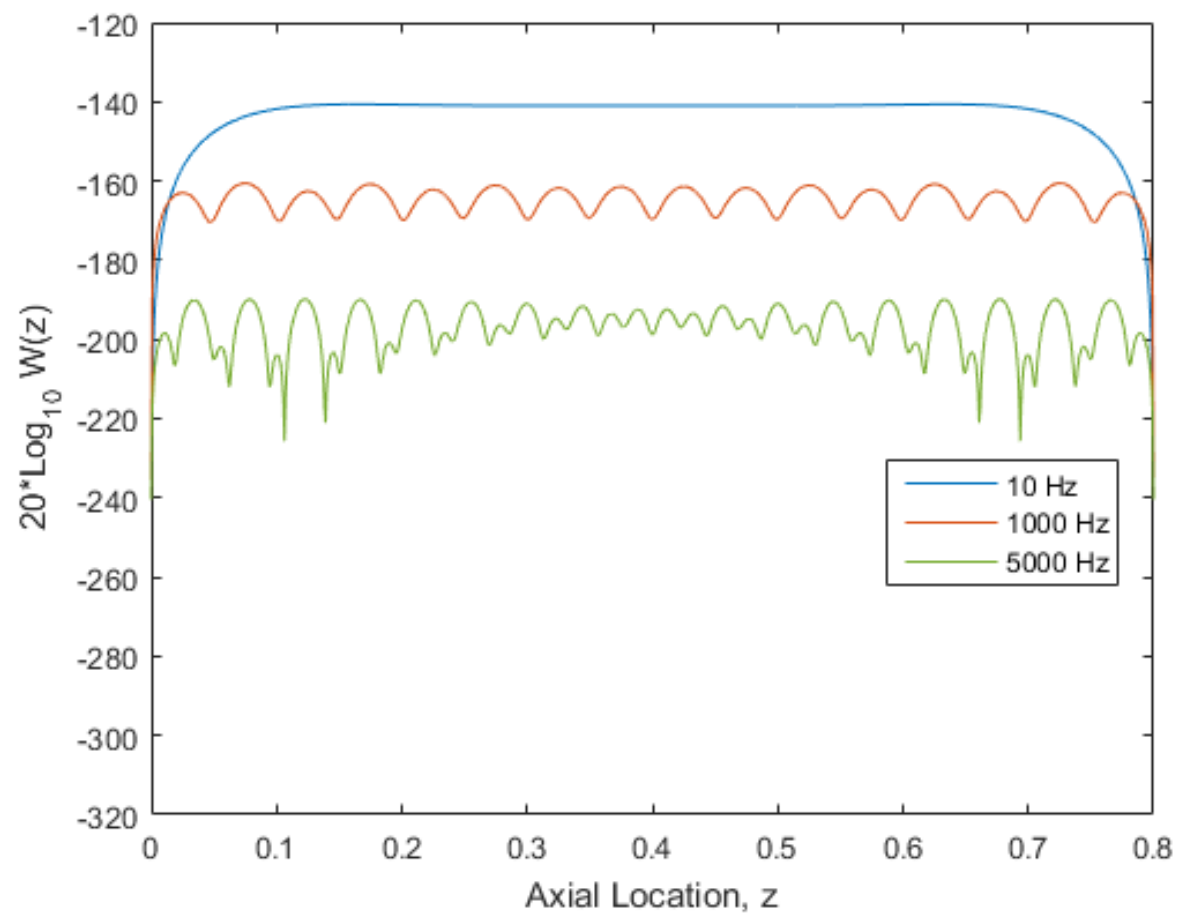

Figure 3.1.14 - Thin-shell radial response for $\operatorname{ring} \operatorname{load}(\mathrm{k}=\omega / \mathrm{cf}, \mathrm{f}=10,1000,5000 \mathrm{~Hz})$

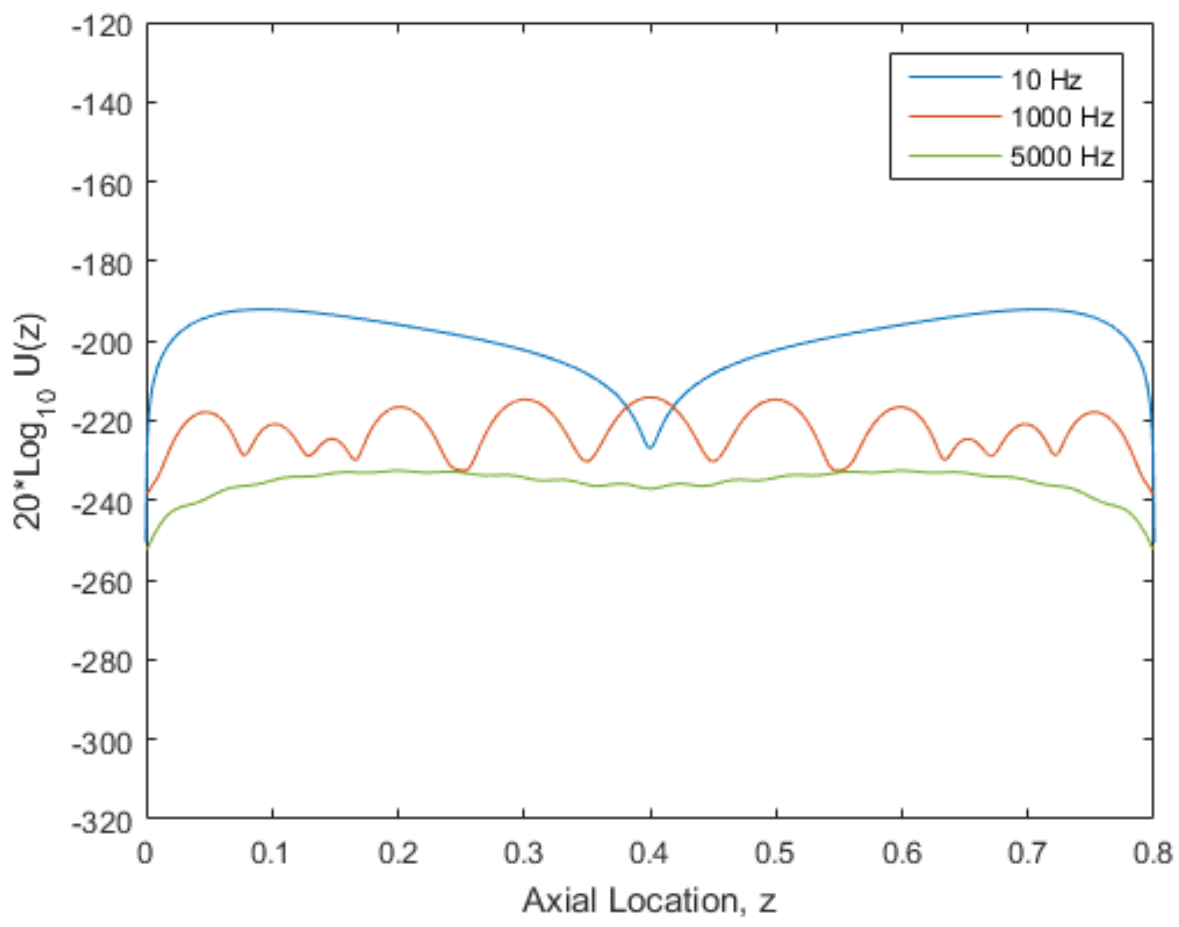

Figure 3.1.15 - Thin-shell axial response for ring load $(\mathrm{k}=\omega / \mathrm{cf}, \mathrm{f}=10,1000,5000 \mathrm{~Hz})$

Periodic reinforcing ribs add significant content compared to the unreinforced model due to the wave reflections from the stiffeners. 
Plane-wave loading at the same oblique angle $\left(\phi_{i}=\pi / 12\right)$ is now applied to the thin-shell system and the unwrapped shell displacements are plotted for a $10 \mathrm{~Hz}$ and $5000 \mathrm{~Hz}$ excitation in Figures 3.1.16 and 3.1.17. This requires additional circumferential modes $(n>0)$, which are more expensive to generate so $n_{p t s}=21$ is chosen.
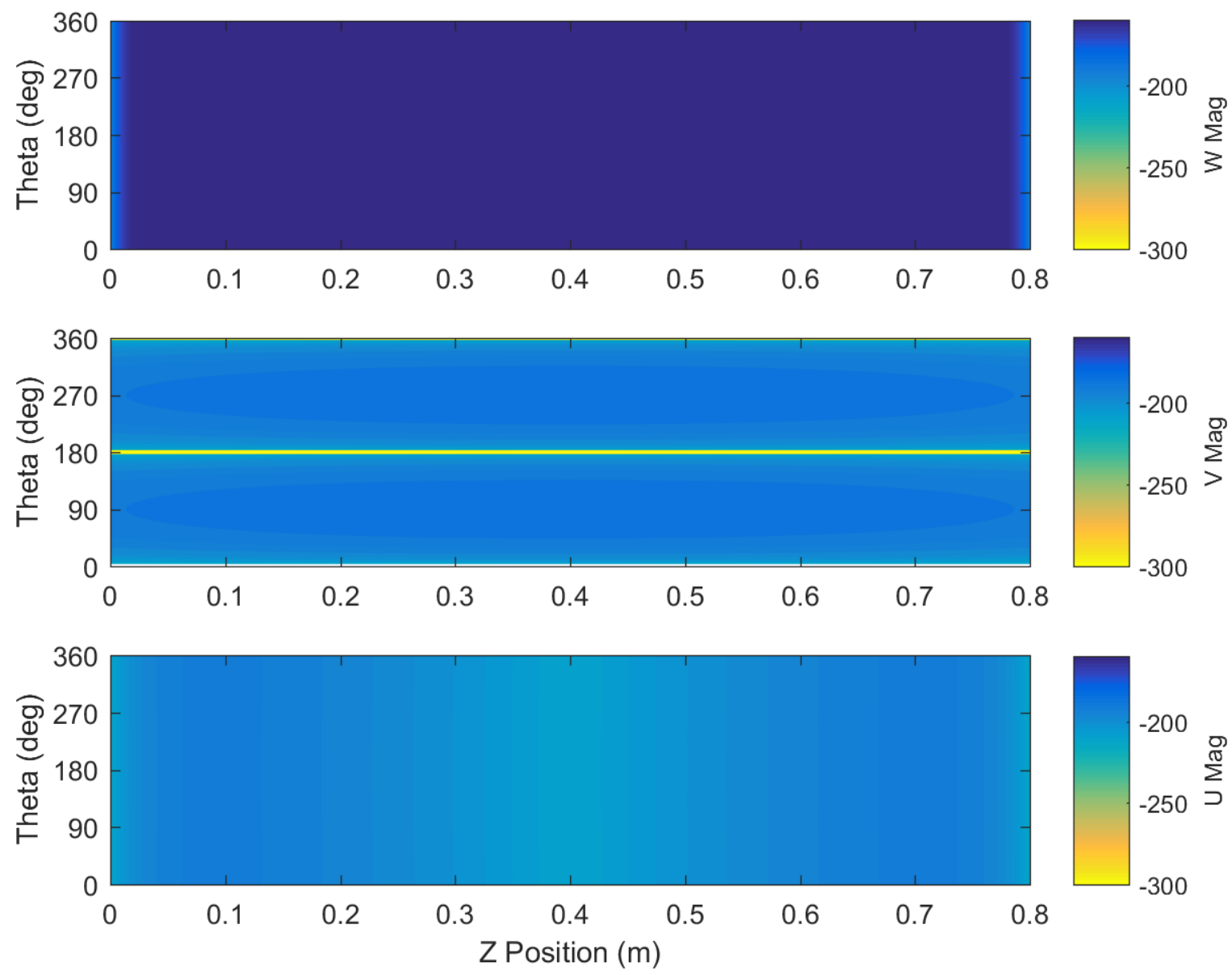

Figure 3.1.16 - Thin-shell cylinder radial (top), tangential (mid), and axial (bottom) displacement response for plane-wave: $10 \mathrm{~Hz}, \varphi \mathrm{i}=\pi / 12, \mathrm{n}_{\mathrm{pts}}=20$ 

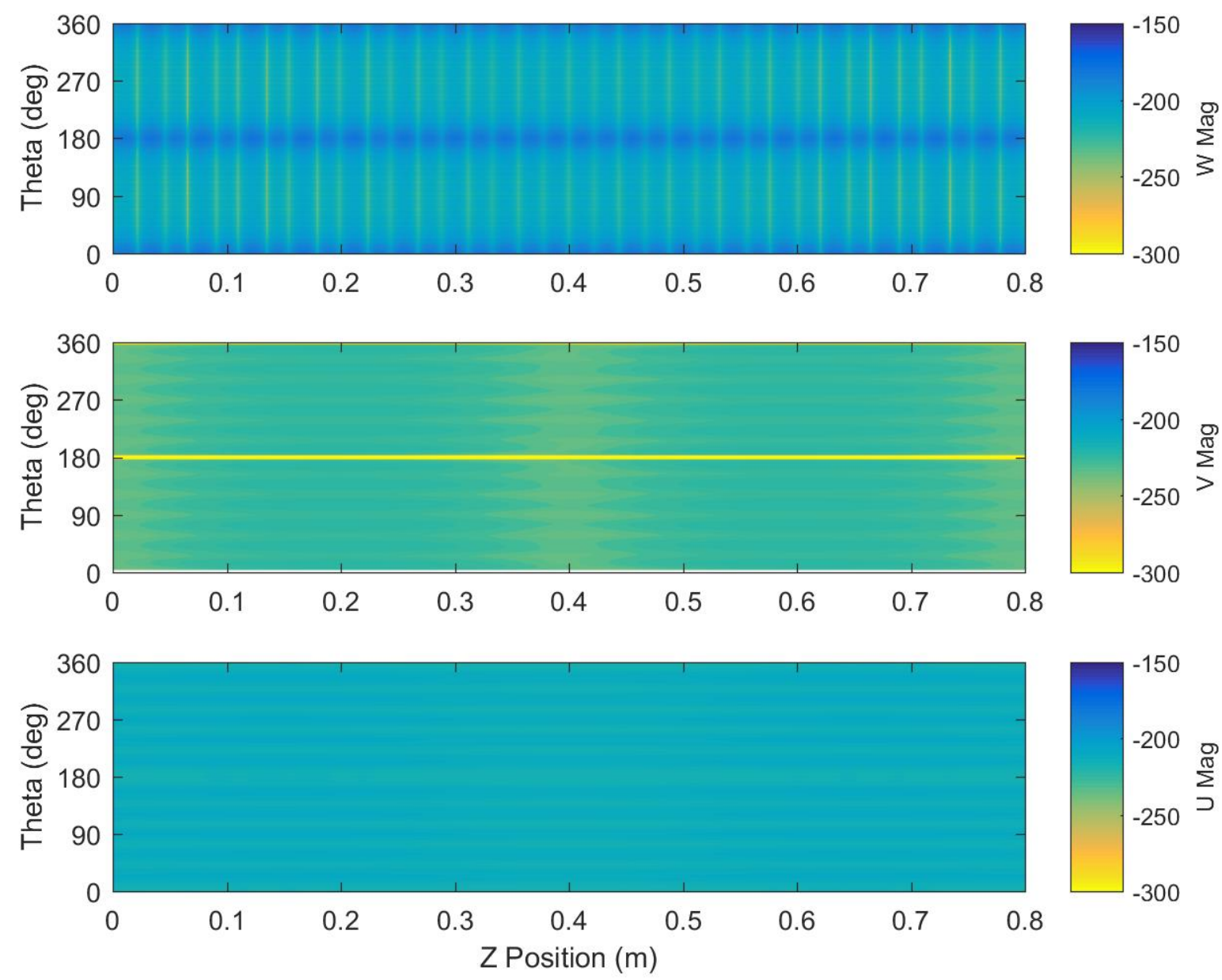

Figure 3.1.17 - Thin-shell cylinder radial (top), tangential (mid), and axial (bottom) displacement response for plane-wave: $5000 \mathrm{~Hz}, \varphi_{\mathrm{i}}=\pi / 12, \mathrm{n}_{\mathrm{pts}}=20$

The low frequency $(10 \mathrm{~Hz})$ response shows a dependency on the z-position of cylinder, but does not have much variation with angular position. The higher frequency response shows a high order circumferential mode and also a longitudinal shear mode seen in the radial displacement (top of 3.1.18).

\subsubsection{Validation of Elastic Model with Thin-Shell}

To support the use of the elastic model, it is necessary to compare it to the thin-shell model. Hull had compared the thin-shell results with a very small thickness, $h=0.001 \mathrm{~m}$, and Figure 3.1.18 shows the unreinforced thin-shell and unreinforced thick-shell models diverging at higher frequencies for larger thicknesses. 

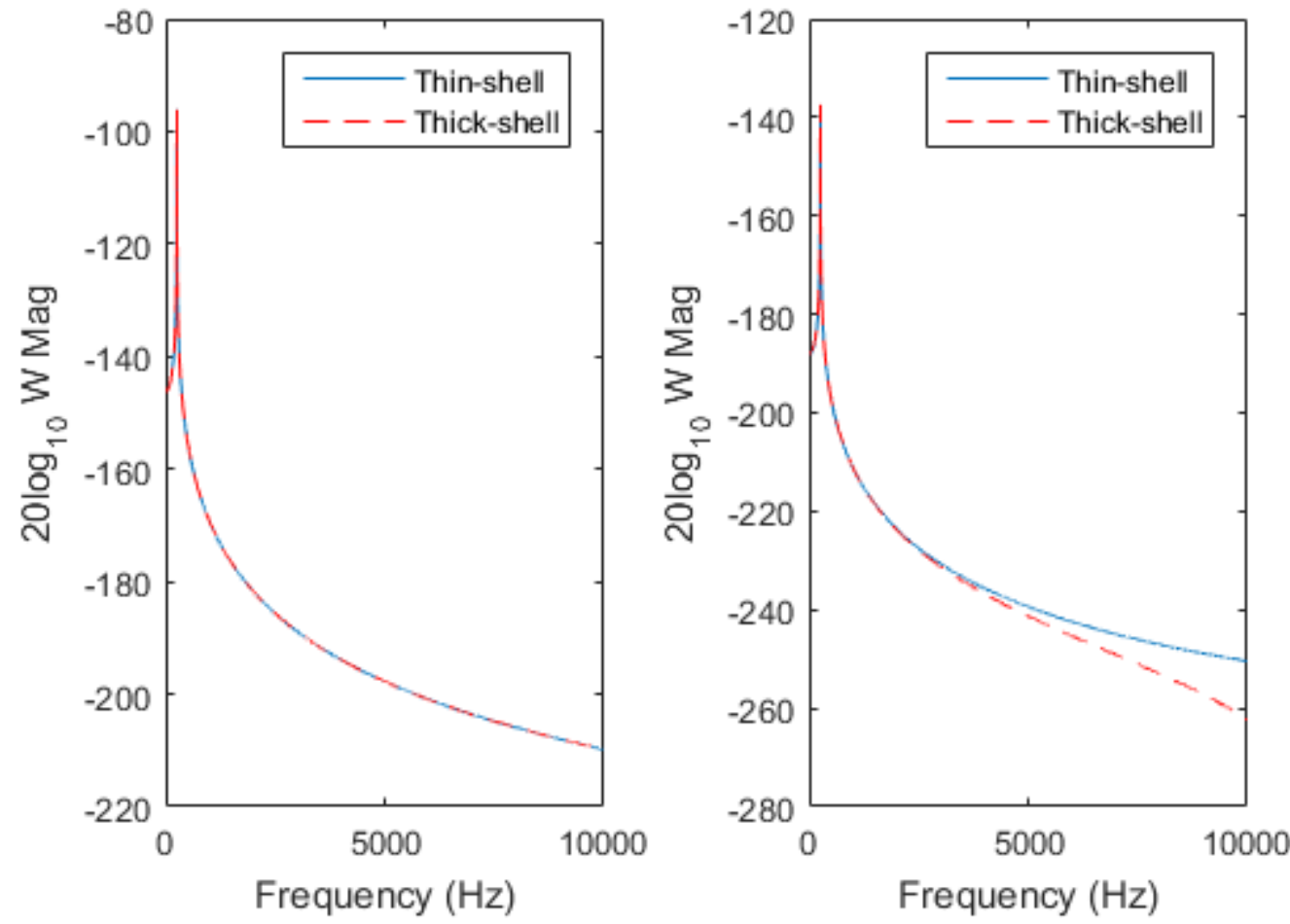

Figure 3.1.18 - Unreinforced models compared for $h=0.001 \mathrm{~m}$ (left) and $\mathrm{h}=0.05 \mathrm{~m}$ (right)

Figure 3.1.18 shows that the two models will diverge for non-small thicknesses $(h>>0.001)$ at higher frequencies ( $f>1000 \mathrm{~Hz}$ ). Therefore, it is necessary to change the thickness of the elastic model to $h=0.001 \mathrm{~m}$, and excite at low frequencies. Another important parameter is the number of stiffener terms, $m_{\mathrm{pts}}$, which must be equal for both models to ensure a fair assessment. Relative to the thin-shell model, evaluating the elastic model is computationally expensive, and thus $\mathrm{m}_{\mathrm{pts}}$ must be kept to a minimum acceptable value. Table 3.1.7 presents the simulation parameters for the elastic validation.

\begin{tabular}{|c|c|c|c|}
\hline Setting & Symbol & Value & Units \\
\hline Input Frequency & $\mathrm{f}$ & $10,50,90$ & $\mathrm{~Hz}$ \\
\hline Input Wavenumber & $\mathrm{k}$ & $2 \pi \mathrm{f} / \mathrm{cf}_{\mathrm{f}}$ & $1 / \mathrm{m}$ \\
\hline Thickness & $\mathrm{h}$ & 0.001 & $\mathrm{~m}$ \\
\hline Number of Circ. Modes & $\mathrm{n}_{\mathrm{pts}}$ & 1 & None \\
\hline Number of Axial Modes & $\mathrm{m}_{\mathrm{pts}}$ & 31 & None \\
\hline Rib Stiffness Radial & $\mathrm{K}_{\mathrm{r}}$ & $1 \times 10^{10}$ & $\mathrm{~N} / \mathrm{m}$ \\
\hline Rib Stiffness Tangential & $\mathrm{K}_{\mathrm{t}}$ & $1 \times 10^{10}$ & $\mathrm{~N} / \mathrm{m}$ \\
\hline Rib Stiffness Axial & $\mathrm{K}_{\mathrm{z}}$ & $1 \times 10^{10}$ & $\mathrm{~N} / \mathrm{m}$ \\
\hline
\end{tabular}

Table 3.1.7 - Elastic and Thin-shell base model settings for validation 
A comparison between the thin-shell and elastic model for 10,50 , and $90 \mathrm{~Hz}$ is presented in Figure 3.1.19 and 3.1.20.
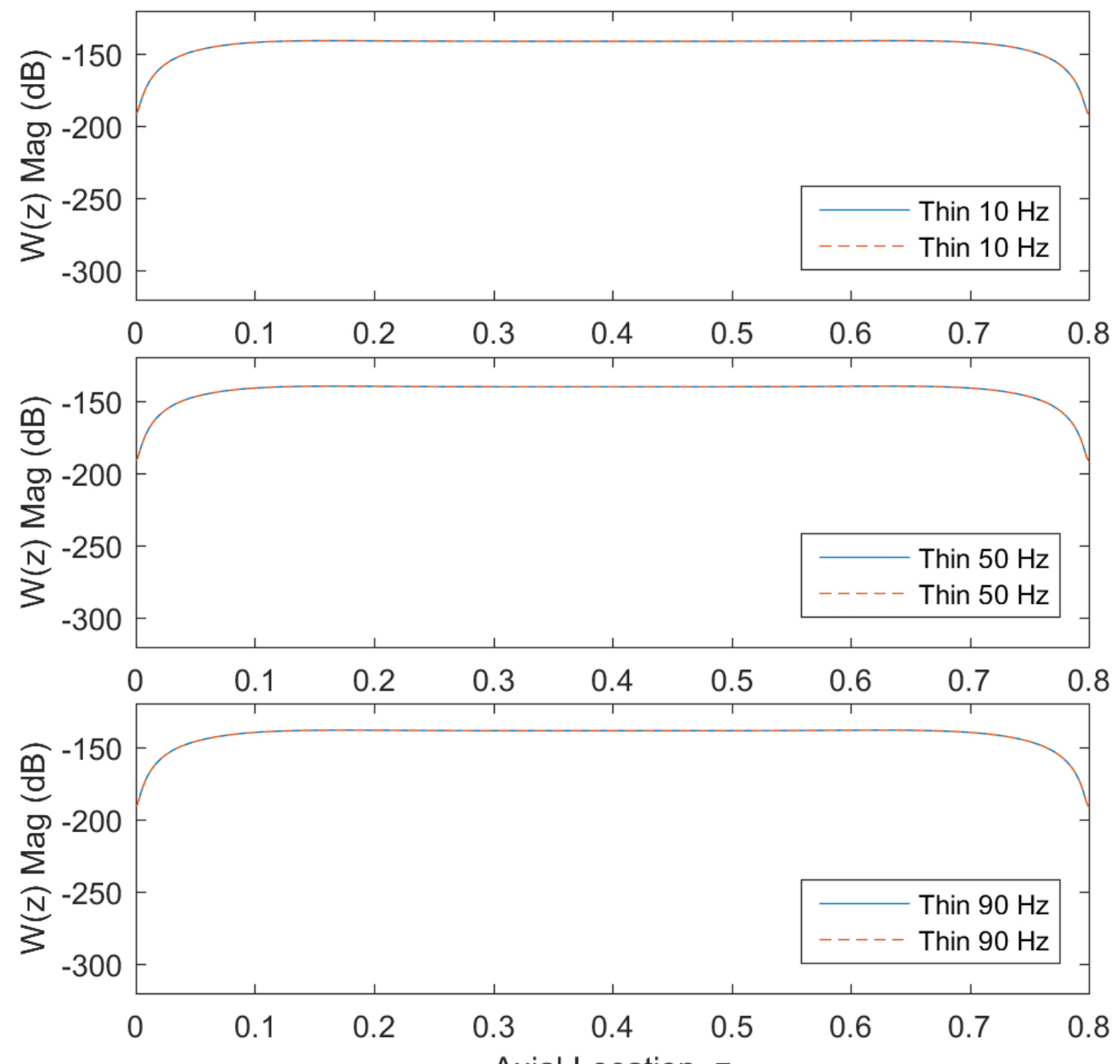

Axial Location, z

Figure 3.1.19 - Comparison of thin and thick-shell $\left(m_{p t s}=31\right)$ radial displacement excited by 10,50 and $90 \mathrm{~Hz}$ Ring Loading $(\mathrm{k}=0)$ 

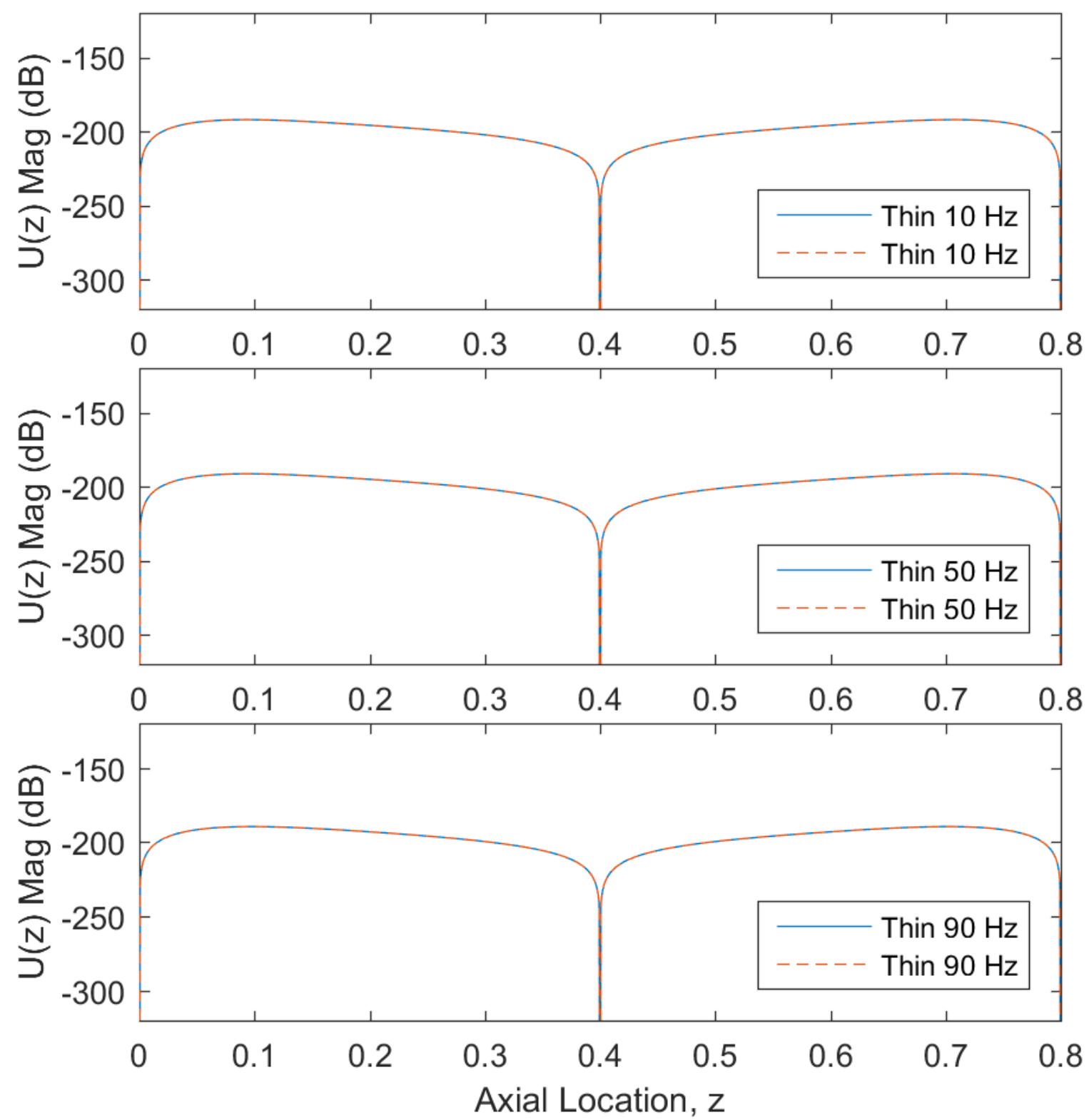

Figure 3.1.20 - Comparison of thin and thick-shell $\left(\mathrm{m}_{\mathrm{pts}}=31\right)$ axial displacement excited by 10,50 and $90 \mathrm{~Hz}$ Ring Loading $(\mathrm{k}=0)$

Both the radial and axial displacement from the elastic model match the reference thin-shell model very well, the magnitudes are nearly indistinguishable. As mentioned, the number of axial modes must be kept to the minimum value that achieves convergence. Plotting the magnitude of each mode shows the diminishing contributions as the index number increases. 


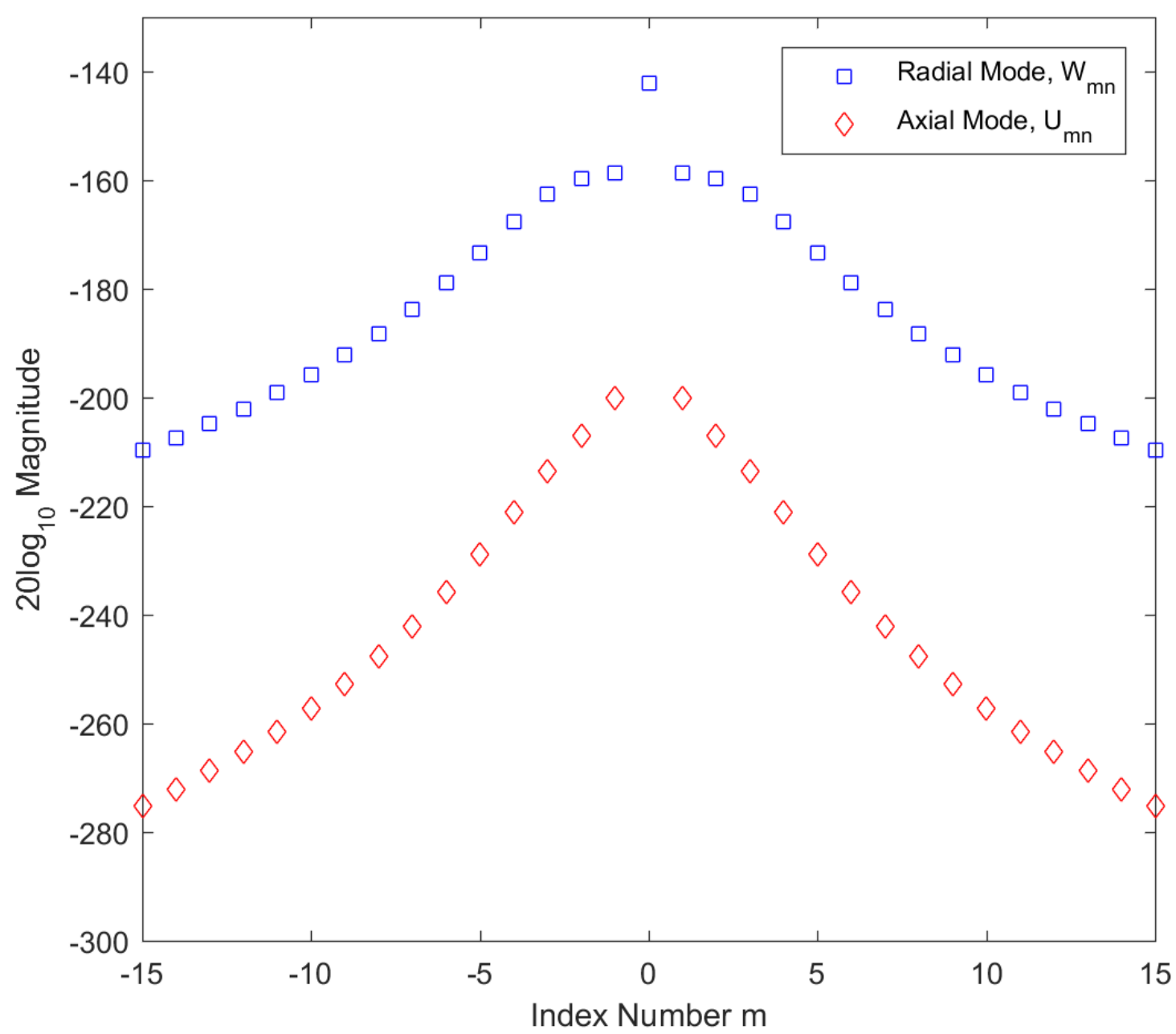

Figure 3.1.21 - Magnitude of displacement modes for each axial index, $m$

For $m_{p t s}=31$, the modes are truncated at $m= \pm 15$, which are significantly smaller than the primary (largest) modes; the magnitude of the $15^{\text {th }}$ axial displacement mode is $-70 \mathrm{~dB}$ less than the primary mode (roughly $3 \times 10^{-4}$ ). Thus, the elastic model is deemed converged at $\mathrm{m}_{\mathrm{pts}}=31$, and is validated by the reference model. Note that there is no appreciable tangential displacement for the symmetric ring load, so it is not shown.

Acoustic plane-wave inputs do not cause a symmetric response and are dependent upon the circumferential mode, $\mathrm{n}$. Therefore, the acoustic wave produces more complex motion and is a more thorough test for validation. It is necessary to compute a nominalized error, which can be displayed as a contour similar to the thin and thick-shell results.

$$
\operatorname{Error}(d B)=20 \log _{10}\left|\frac{\left|(W, V, U)_{\text {thin }}-(W, V, U)_{\text {thick }}\right|}{\left|(W, V, U)_{\text {thin }}\right|}\right|
$$



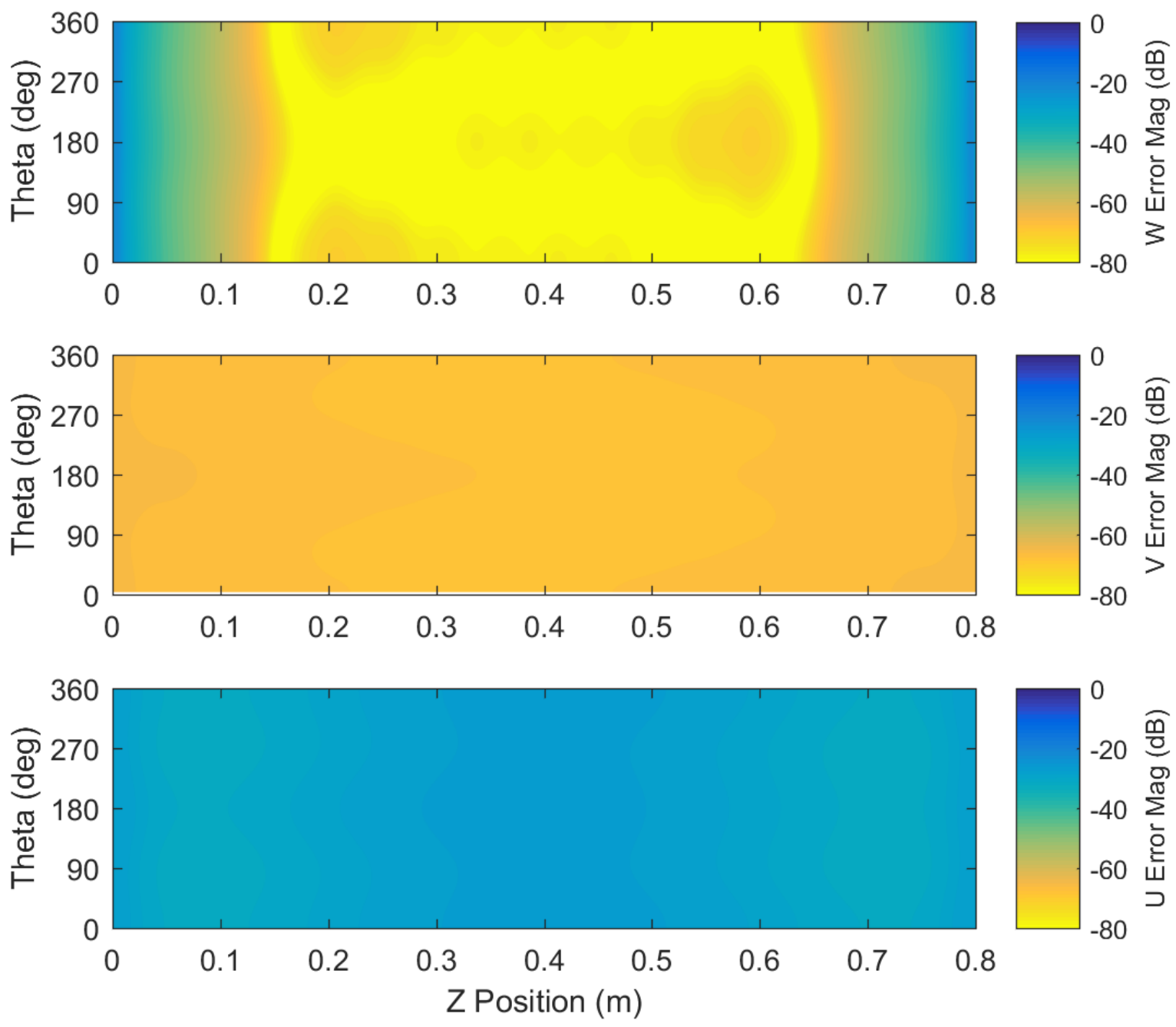

Figure 3.1.22 - Thin and Thick-shell radial (top), tangential (mid), and axial (bottom)

Normalized Error in $\mathrm{dB}$ for plane-wave: $50 \mathrm{~Hz}, \varphi \mathrm{i}=\pi / 12$, mpts $=31$, npts $=7$

Figure 3.1.22 presents the normalized error, in which a value of 0 indicates $100 \%$ error and $-80 \mathrm{~dB}$ indicates $0.01 \%$ error. Axial displacement error has a peak level of $-30 \mathrm{~dB}$ which is roughly $4 \%$ error, which is satisfactory considering the small amplitude scales; the radial and tangential error is less than $1 \%$. Convergence of the thick-shell solution is determined by investigating the relative magnitude of the $m$ and $n$ modes. Figure 3.1.23 to 3.1.25 display the mode contributions for the axial modes, circumferential modes and the normalized 2-dimensional contour, respectively. 


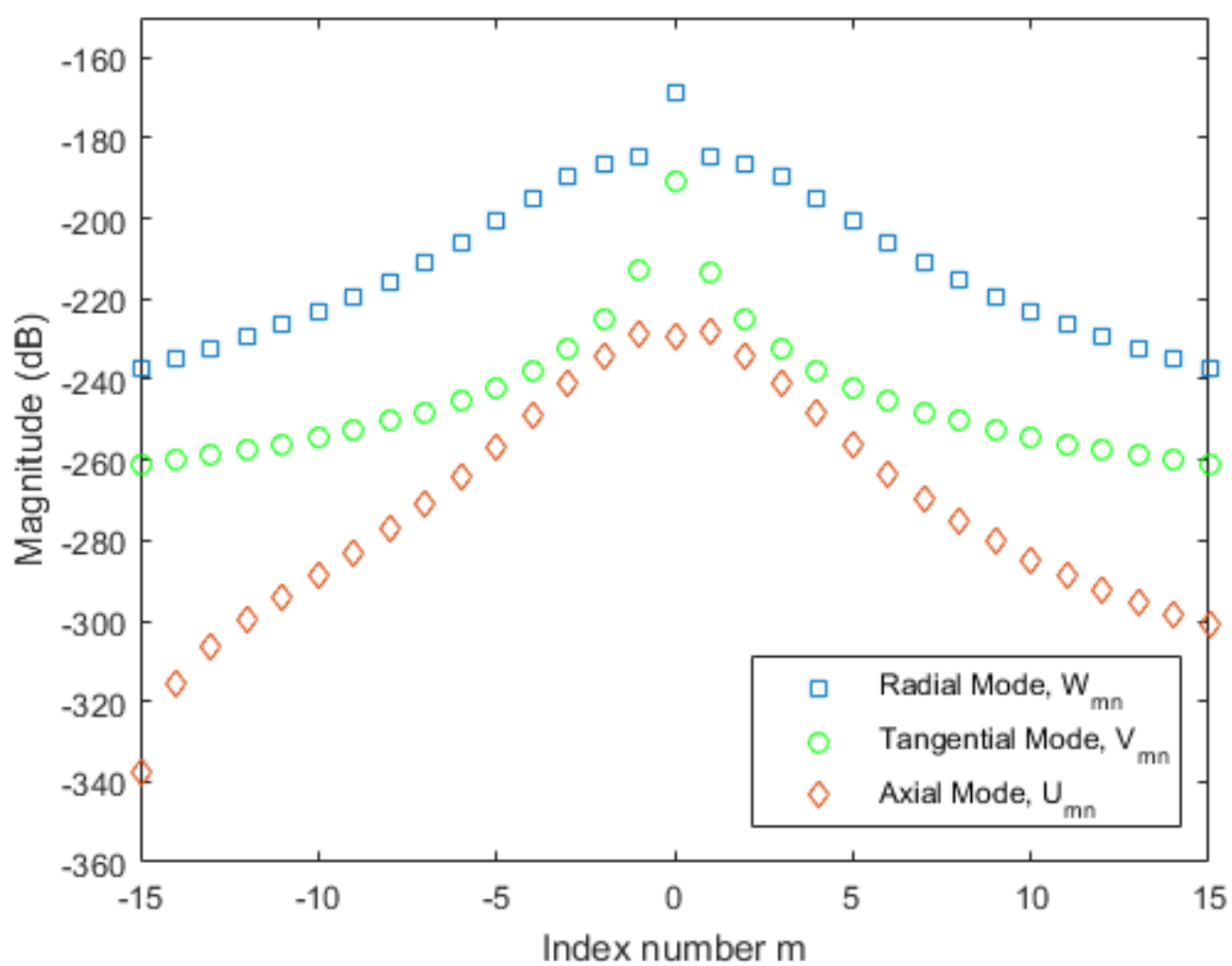

Figure 3.1.23 - Magnitude of displacement modes for each axial index, $m$

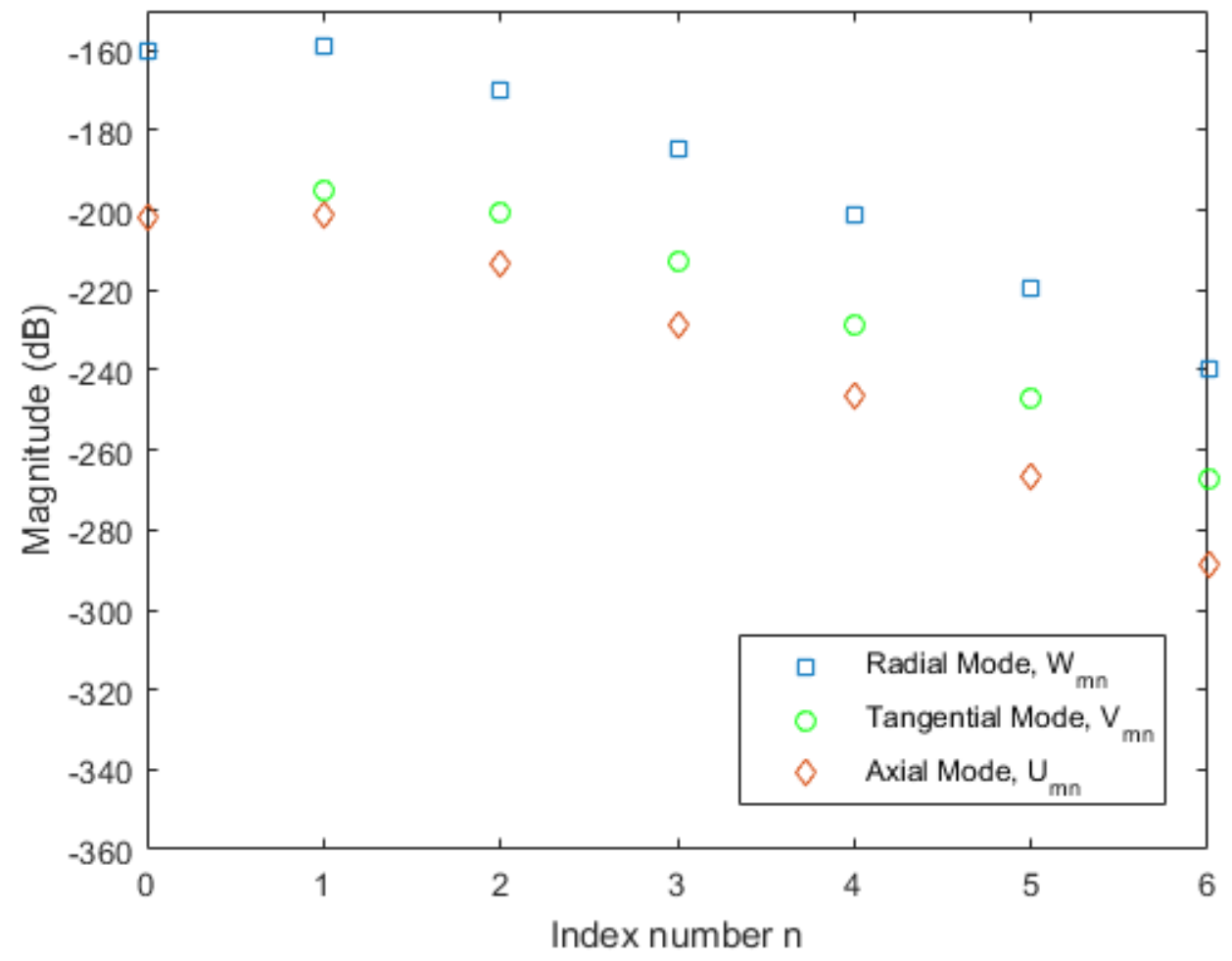

Figure 3.1.24 - Magnitude of displacement modes for each circumferential index, $n$ 

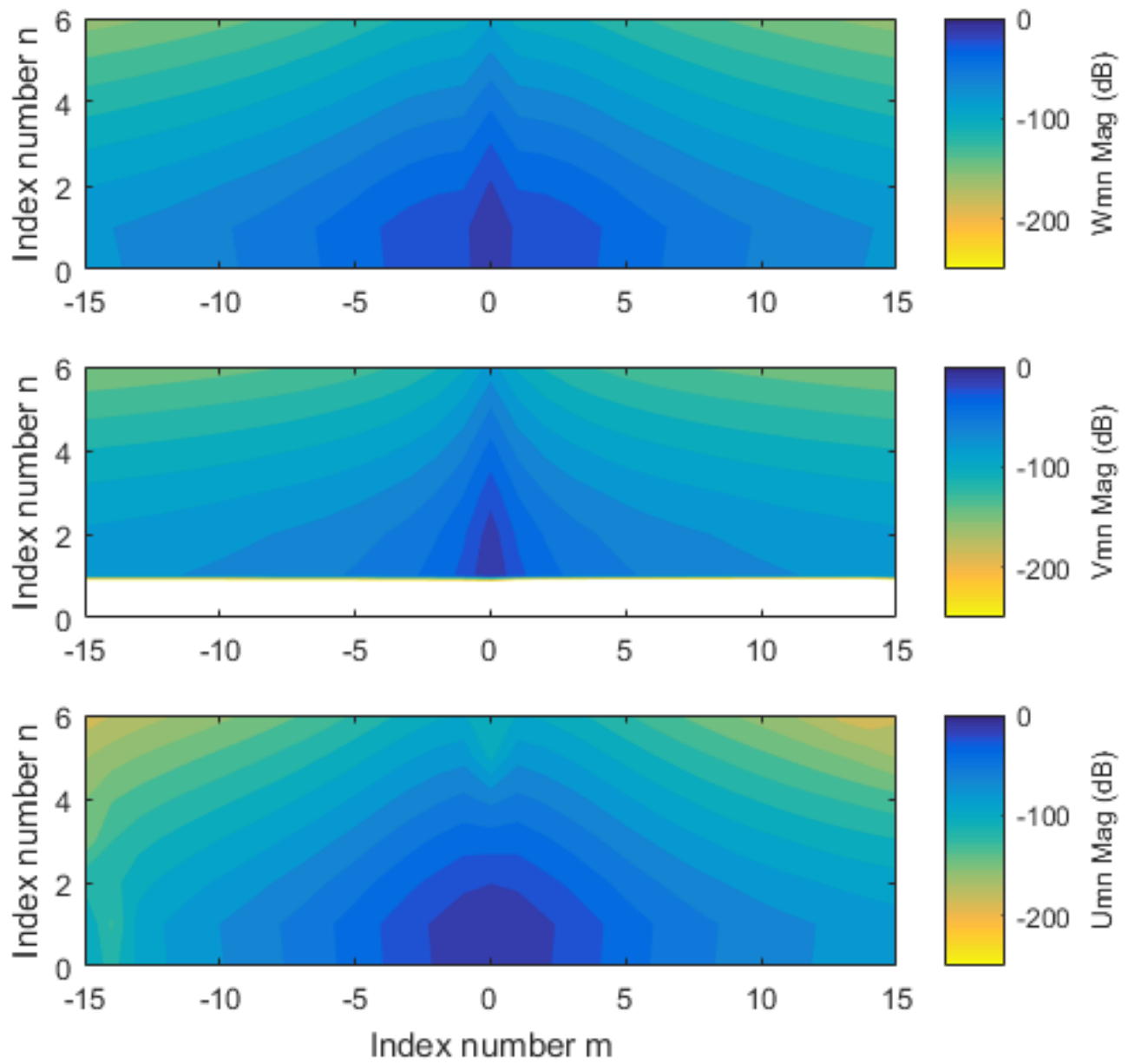

Figure 3.1.25 - Normalized magnitude of displacement modes for each circumferential index, $n$ and axial index, $m$

There is at least a $60 \mathrm{~dB}$ reduction in magnitude from the largest mode to the smallest for each displacement, indicating that the simulation has converged. Consequently, $\mathrm{m}_{\mathrm{pts}}$ and $\mathrm{n}_{\mathrm{pts}}$ will be set to 31 and 7, respectively, for the following simulations.

\subsubsection{Base Model Displacement Response}

With the elastic model validated, the thickness can be increased back to the nominal value, $\mathrm{h}=0.0254 \mathrm{~m}$, and higher frequencies can be investigated. Shell displacements are output at the mid-point radius of the shell thickness (matching the thin-shell output radius); the behavior of the shell throughout its thickness will be investigated in the final model with coating. First the base model ring-loading response is shown in Figures 3.1.26-3.1.27. 


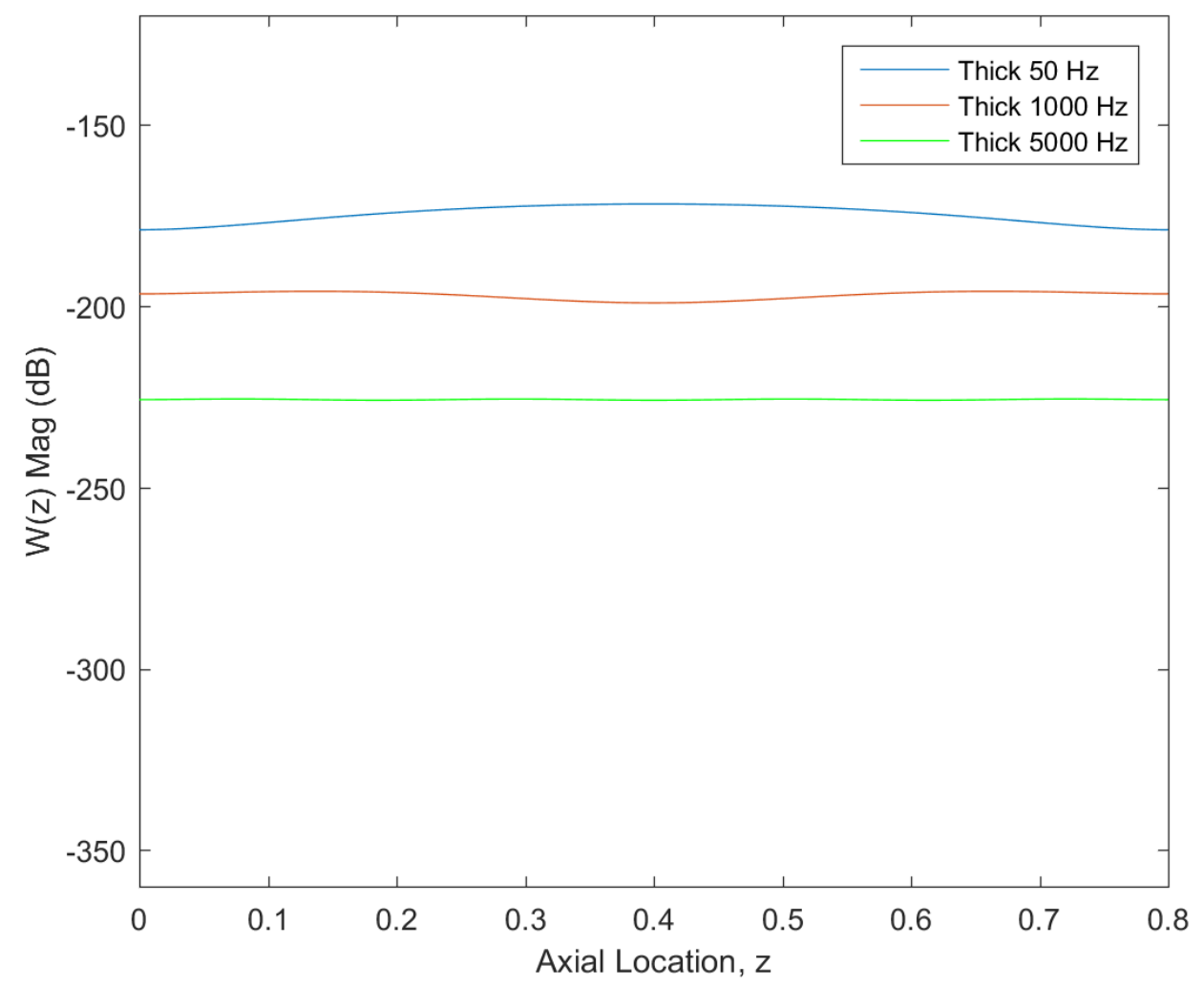

Figure 3.1.26 - Base model radial displacement response excited by 50, 1000 and $5000 \mathrm{~Hz}$ Ring Loading $(\mathrm{k}=0)$

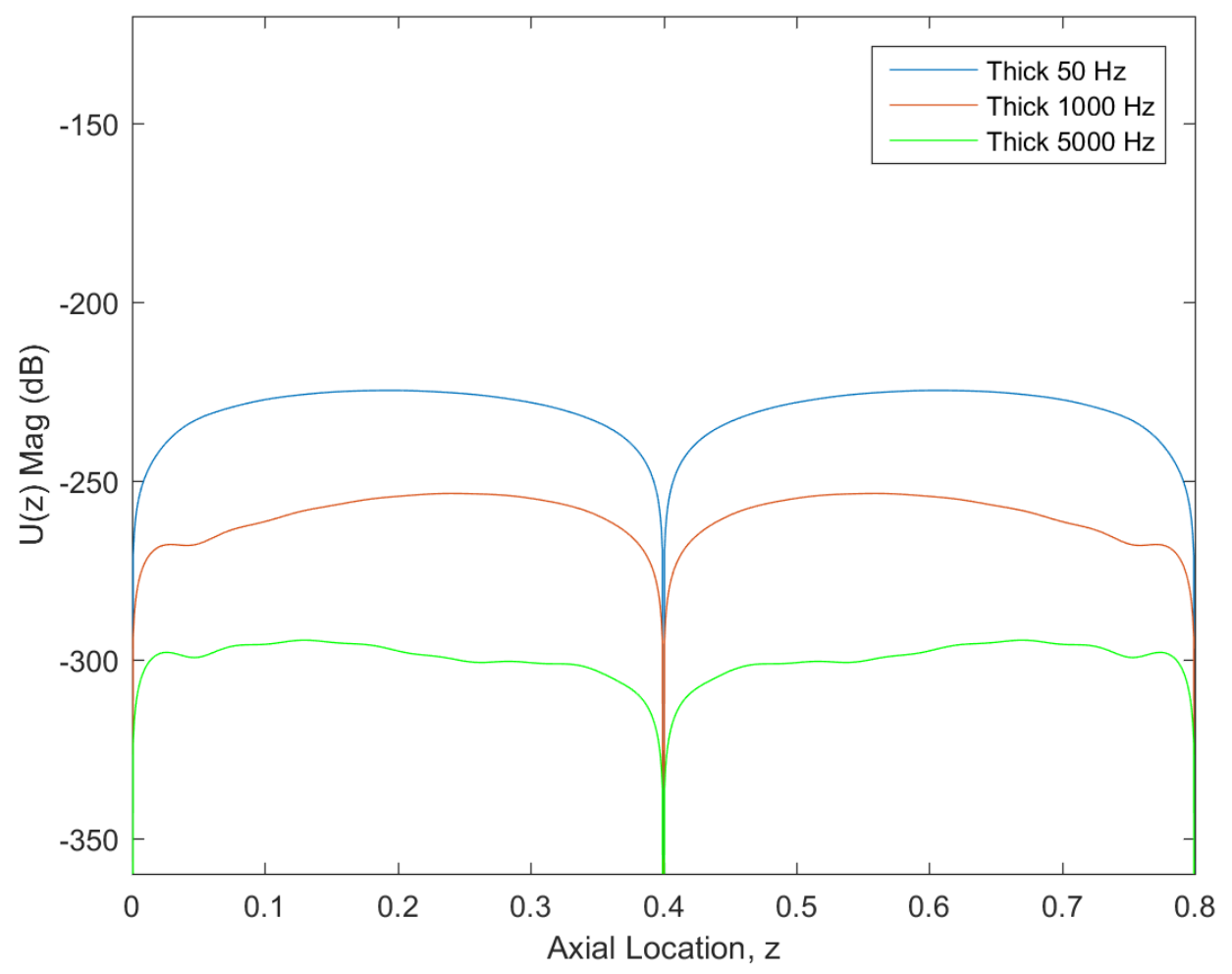

Figure 3.1.27 - Base model axial displacement response excited by 50, 1000 and $5000 \mathrm{~Hz}$ Ring Loading $(\mathrm{k}=0)$ 
Increasing the thickness has a large effect on the structure stiffness, and the displacement magnitudes are much lower than before. Also, the radial response has very little variation along the $Z$ axis; the slight sinusoidal amplitude is hidden by the plot scales.

The base model is also excited with an acoustic plane-wave. Results and are presented as 2-dimensional contours of magnitude in Figures 3.1.28 - 3.1.30.
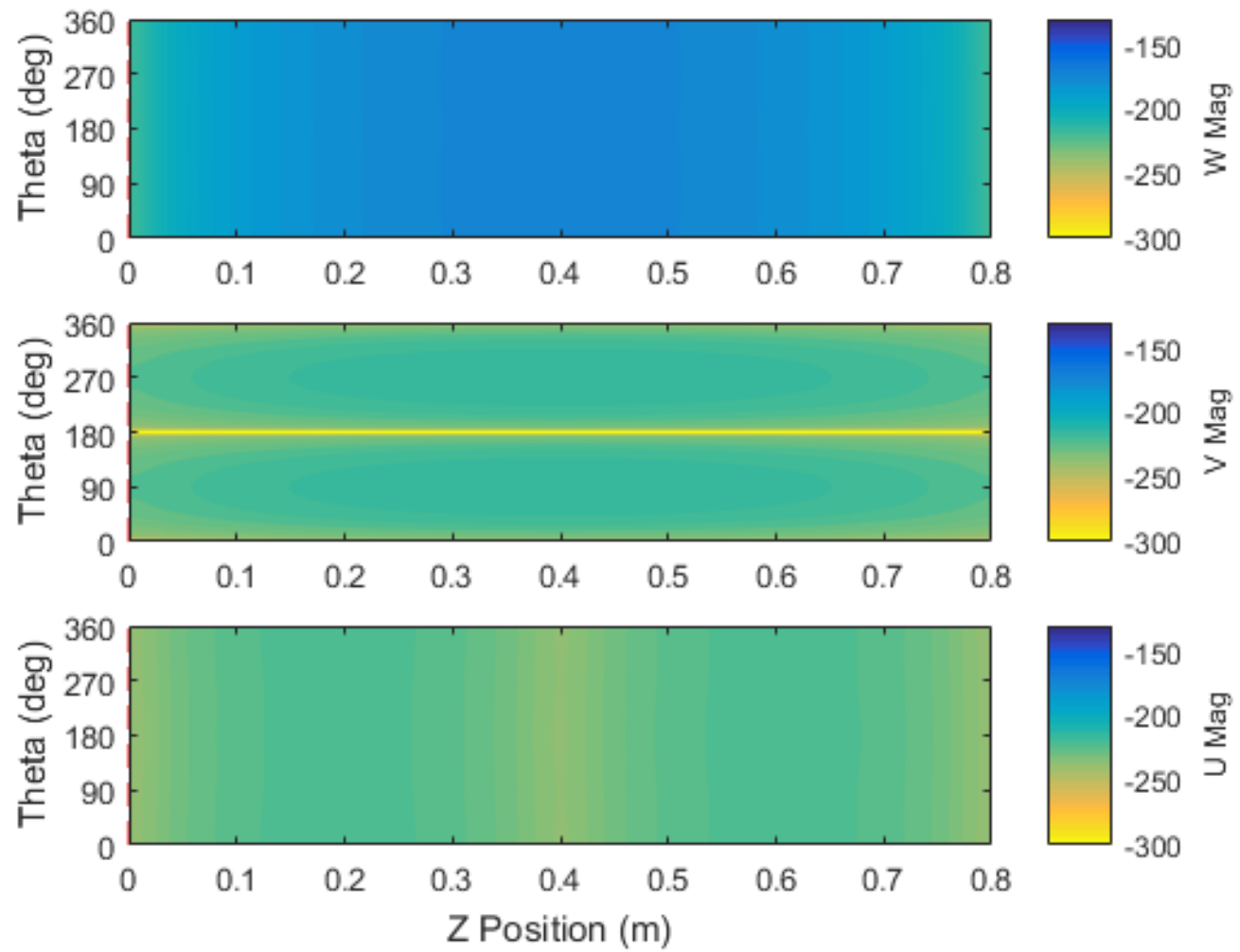

Figure 3.1.28 - Elastic base model radial (top), tangential (mid), and axial (bottom) displacement response for acoustic-wave: $50 \mathrm{~Hz}, \varphi_{\mathrm{i}}=15 \mathrm{deg}$ of broadside, $\mathrm{m}_{\mathrm{pts}}=31, \mathrm{n}_{\mathrm{pts}}=7$ 

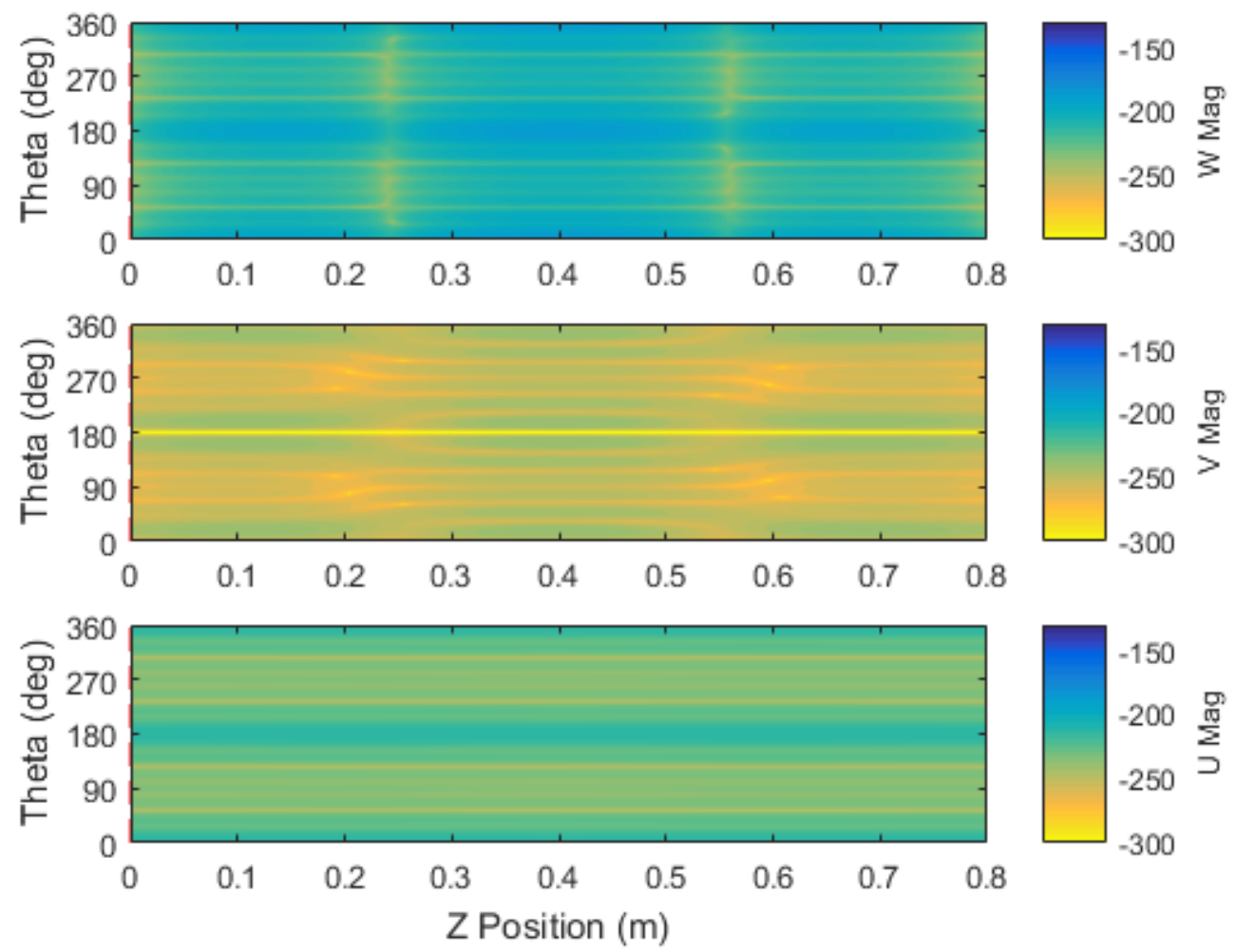

Figure 3.1.29 - Elastic base model radial (top), tangential (mid), and axial (bottom) displacement response for acoustic-wave: $1000 \mathrm{~Hz}, \varphi_{\mathrm{i}}=15 \mathrm{deg}, \mathrm{m}_{\mathrm{pts}}=31, \mathrm{n}_{\mathrm{pts}}=7$
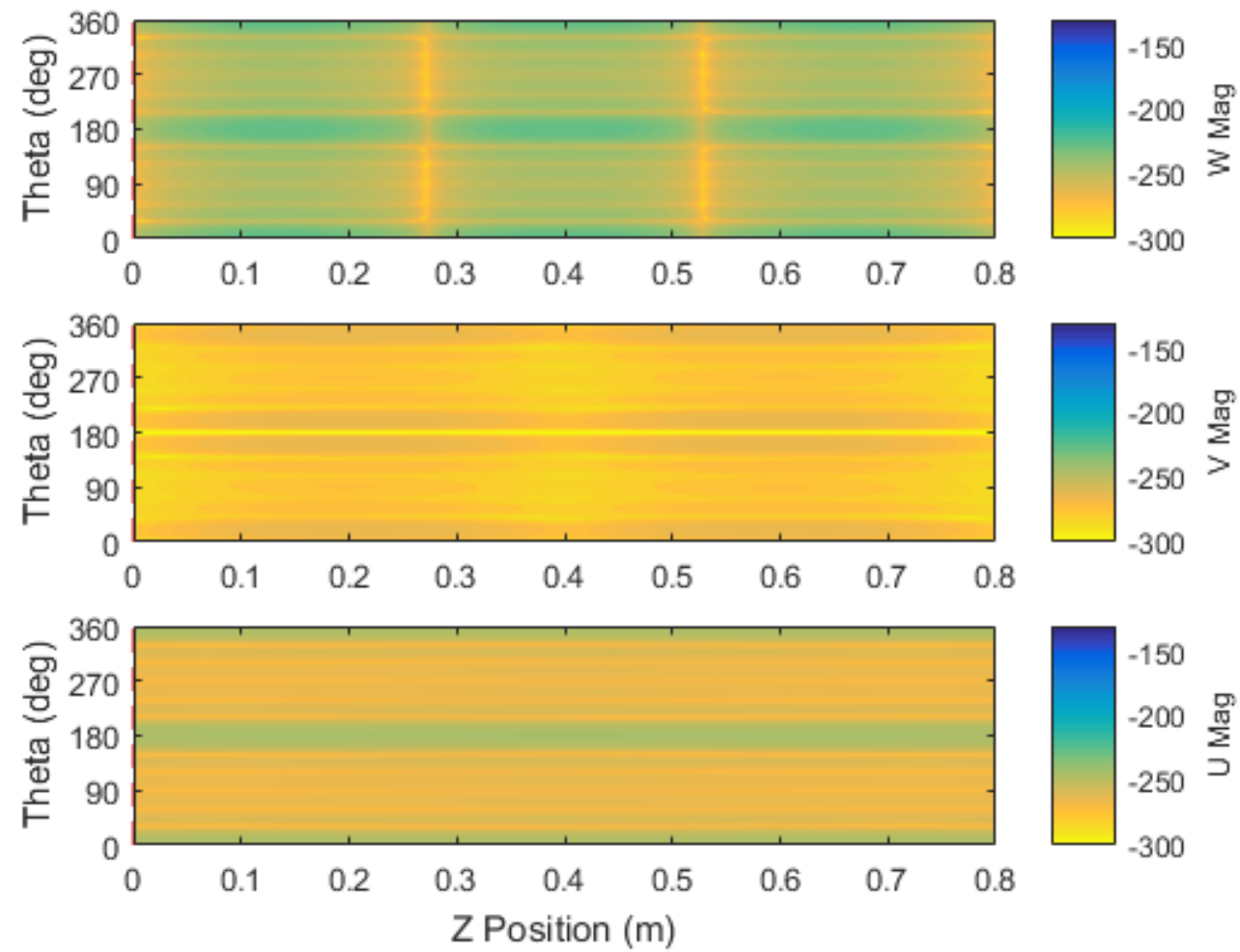

Figure 3.1.30 - Elastic base model radial (top), tangential (mid), and axial (bottom) displacement response for acoustic-wave: $5000 \mathrm{~Hz}, \varphi_{\mathrm{i}}=15 \mathrm{deg}, \mathrm{m}_{\mathrm{pts}}=31, \mathrm{n}_{\mathrm{pts}}=7$ 
A unique feature of the acoustic wave, which impacts the shell at $\theta=0$, is that the tangential displacement (V) has zero magnitude at $\theta=0$ and $\theta=180$; as expected, all 3 displacements are symmetric about the $\theta=180$ horizontal line. At $50 \mathrm{~Hz}$ excitation, the radial and axial displacements express a low wavenumber sine wave traveling along the $Z$ axis. At higher input frequencies, the motion is dominated by dilatational and shear circumferential modes, presented by the lines of constant magnitude propagating along the $Z$ axis. Generally, the displacement magnitude lessens with increasing excitation frequency.

\subsection{Numerical Challenges}

\subsubsection{Bessel Function Evaluations}

Bessel functions and the related Hankel functions are an integral part of developing the elasticity solution for cylindrical solids, with or without reinforcement. The separation of variables method eventually leads to the Bessel's equation in terms of the radial variable, $r$, and circumferential index, $n$. Bessel functions are the solutions to this equation. Development of the unreinforced elastic cylindrical shell concludes with a formulation of the displacement field coefficients in terms of Bessel functions (Equation 2.1.52 - 2.1.54):

$$
J_{n}(\alpha r), \quad Y_{n}(\alpha r), \quad J_{n}(\beta r), \quad Y_{n}(\beta r)
$$

where $\alpha$ and $\beta$ are the modified wavenumbers given by

$$
\alpha=\sqrt{\frac{\omega^{2}}{c_{1}^{2}}-k^{2}}, \quad \beta=\sqrt{\frac{\omega^{2}}{c_{2}^{2}}-k^{2}}
$$

If the argument is imaginary, the modified Bessel functions are used. Figure 2.1.3 shows that the modified Bessel functions become asymptotic for arguments approaching zero (K type) or arguments away from zero (I type).

When reinforcement is considered, the modified wavenumbers are indexed with the axial mode, $m$, to produce the following

$$
\begin{aligned}
& \alpha_{\mathrm{m}}=\sqrt{\frac{\omega^{2}}{\mathrm{c}_{1}^{2}}-\mathrm{k}_{\mathrm{m}}^{2}}=\sqrt{\frac{\omega^{2}}{\mathrm{c}_{1}^{2}}-\left(\mathrm{k}_{\mathrm{z}}+\frac{2 \pi \mathrm{m}}{\mathrm{L}}\right)^{2}}, \\
& \beta_{\mathrm{m}}=\sqrt{\frac{\omega^{2}}{\mathrm{c}_{2}^{2}}-\mathrm{k}_{\mathrm{m}}^{2}}=\sqrt{\frac{\omega^{2}}{\mathrm{c}_{2}^{2}}-\left(\mathrm{k}_{\mathrm{z}}+\frac{2 \pi \mathrm{m}}{\mathrm{L}}\right)^{2}}
\end{aligned}
$$


For higher order $\mathrm{m}$, the modified wavenumbers become imaginary with large amplitude, thus they can certainly pose numerical problems once the Bessel functions are evaluated, unless $L$, the periodic rib spacing, is unrealistically large.

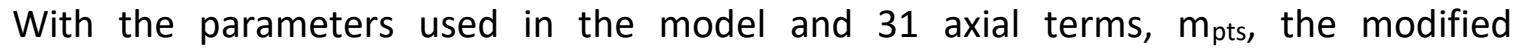
wavenumbers reach approximately $\mathbf{1 2 0 . 0 i}$, which evaluates to $1 \mathbf{e 2 3 0}$ from the Bessel functions. Understandably, this will cause serious issues when solving the matrix equation. Unfortunately using asymptotic approximate forms of the Bessel functions produces similarly large numbers.

\subsubsection{III-conditioned A Matrix}

After forming the global matrices for the shell dynamics $(A)$ and the stiffener stresses $(Z$, $T$, and R), the matrix equation must be solved, as seen in Equation 2.3.32 and Equation 2.4.47.

$$
\widehat{\boldsymbol{x}}_{\boldsymbol{n}}=\left[\widehat{\boldsymbol{A}}_{\boldsymbol{n}}+\frac{K_{z}}{a b} \widehat{\boldsymbol{Z}}_{\boldsymbol{n}}+\frac{K_{t}}{a b} \widehat{\boldsymbol{T}}_{\boldsymbol{n}}+\frac{K_{r}}{a b} \widehat{\boldsymbol{R}}_{\boldsymbol{n}}\right]^{-1} \widehat{\boldsymbol{f}}_{\boldsymbol{n}}
$$

Generally, this matrix equation can be considered as the standard matrix equation

$$
\left[\widehat{\boldsymbol{A}}_{\boldsymbol{n}}+\frac{K_{z}}{a b} \widehat{\boldsymbol{Z}}_{\boldsymbol{n}}+\frac{K_{t}}{a b} \widehat{\boldsymbol{T}}_{\boldsymbol{n}}+\frac{K_{r}}{a b} \widehat{\boldsymbol{R}}_{\boldsymbol{n}}\right] \widehat{\boldsymbol{x}}_{\boldsymbol{n}}=\widehat{\boldsymbol{f}}_{\boldsymbol{n}} \rightarrow \boldsymbol{A} \boldsymbol{x}=\boldsymbol{b}
$$

which is not solved by finding the matrix inverse of $\mathbf{A}$. Instead, more efficient methods exist and are used by the Matlab "mldivide" operator, based on the properties of the A matrix [24]. For the thin-shell reference model, with a ring loading input of magnitude $P_{0}=1$ :

$$
[A]\left[\begin{array}{c}
\vdots \\
x_{-2} \\
x_{-1} \\
x_{0} \\
x_{1} \\
x_{2} \\
\vdots
\end{array}\right]=\left[\begin{array}{c}
\vdots \\
0 \\
0 \\
b^{\prime} \\
0 \\
0 \\
\vdots
\end{array}\right], \quad x_{m}=\left[\begin{array}{c}
U_{m} \\
V_{m} \\
W_{m}
\end{array}\right], \quad b^{\prime}=\left[\begin{array}{l}
0 \\
0 \\
1
\end{array}\right]
$$

Each equation/row of the thin-shell model represents the 3 stress equations, $\left[\begin{array}{lll}\tau_{\mathrm{rr}} & \tau_{\mathrm{r} \theta} & \tau_{\mathrm{rz}}\end{array}\right]^{\mathrm{T}}$. For the thick-shell with the same input:

$$
[A]\left[\begin{array}{c}
\vdots \\
x_{-2} \\
x_{-1} \\
x_{0} \\
x_{1} \\
x_{2} \\
\vdots
\end{array}\right]=\left[\begin{array}{c}
\vdots \\
0 \\
0 \\
b^{\prime} \\
0 \\
0 \\
\vdots
\end{array}\right], \quad x_{m}=\left[\begin{array}{c}
A_{m} \\
B_{m} \\
C_{m} \\
D_{m} \\
E_{m} \\
F_{m}
\end{array}\right], \quad b^{\prime}=\left[\begin{array}{l}
0 \\
0 \\
0 \\
1 \\
0 \\
0
\end{array}\right]
$$

Each equation/row of the thick-shell model represents the 3 stress equations at the 2 boundary conditions (inner surface $r=a$, and outer surface $r=c$ ) resulting in 6 total equations, 
$\left[\begin{array}{llllll}\tau_{\mathrm{rr}, \mathrm{a}} & \tau_{\mathrm{r} \theta, \mathrm{a}} & \tau_{\mathrm{rz}, \mathrm{a}} & \tau_{\mathrm{rr}, \mathrm{c}} & \tau_{\mathrm{r} \theta, \mathrm{c}} & \tau_{\mathrm{rz}, \mathrm{c}}\end{array}\right]^{\mathrm{T}}$. The solved coefficients, $\mathrm{A}_{\mathrm{m}}$ through $\mathrm{F}_{\mathrm{m}, \text { are then input }}$ into Equation 2.1.52 - 2.1.54 to get the shell displacements, $U_{m} V_{m}$ and $W_{m}$. Recall that the global A matrix of 3.2.6 and 3.2.7 is for each circumferential mode.

The A matrix has the following properties: Non-Hermitian, Non-orthogonal, Non-symmetric, Non-sparse, Square. Mldivide will use an LU factorization technique to numerically solve $A x=b$. Unfortunately, the matrix is severely ill-conditioned:

$$
\operatorname{cond}(A)=10^{240}
$$

Unsurprisingly, any conventional solution method fails when presented a matrix with such a high condition number. Thus, it is not possible to solve the global matrix equation $\mathbf{A x}=\mathbf{b}$ using standard double precision.

\subsubsection{Multi-precision Toolbox}

In order to solve Equation 3.2.5, it is necessary to increase the numerical precision available. The Advanpix Multi-Precision Toolbox integrates into Matlab and provides a library of classes and functions that operate with user-defined precision [25]. All of the Bessel and Hankel functions are present in the library, as well as the mldivide linear system solver. Even though the matrix is so poorly conditioned, with a high enough precision it can still be solved at the expense of simulation run-time.

\subsubsection{Simulation Performance}

Thus there is a balancing act of including enough modes, $m_{p t s}$ such that the simulation has properly converged, and keeping the simulation run-time reasonable. Adding more modes not only affects the condition number of the A matrix and the precision required to solve the matrix equation, but it also increases the size of the system, which is equal to $6 m_{\text {pts }} \times 6 m_{\text {pts. }}$. When

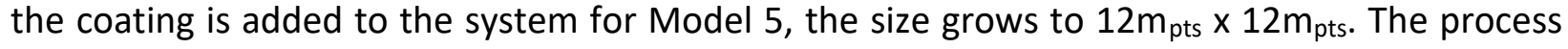
repeats for each circumferential mode, $n$, such that the $A x=b$ must be solved $n_{\text {pts }}$ times for each solution at a single excitation frequency, $\omega$. Table 3.2.1 displays the number of modes, the precision required and the simulation run-time for the base model. 


\begin{tabular}{|c|c|c|}
\hline $\mathrm{m}_{\mathrm{pts}}$ & Precision (digits) & Run-time (sec) \\
\hline 1 & 16 (double) & $<1$ \\
\hline 3 & 64 & 1.5 \\
\hline 7 & 128 & 5.5 \\
\hline 11 & 170 & 18 \\
\hline 15 & 256 & 53 \\
\hline 21 & 340 & 183 \\
\hline 25 & 500 & 409 \\
\hline 31 & 560 & 980 \\
\hline
\end{tabular}

Table 3.2.1 - Simulation performance, Base model

Simulation run-times shown in Table 3.2.1 are for the base model with a single circumferential mode and a single excitation frequency. The coated elastic model, derived in Chapter 4.4 requires a $2 x$ larger matrix system to be solved. This system, consequently, has much longer running times for each configuration. Fortunately, the matrix condition number doesn't change significantly for each value of $m_{\text {pts }}$ between the base and coated model, so the same precision can be used. Table 3.2.2 displays the coated model simulation performance.

\begin{tabular}{|c|c|c|}
\hline $\mathrm{m}_{\mathrm{pts}}$ & Precision (digits) & Run-time (sec) \\
\hline 1 & 16 (double) & $<1$ \\
\hline 3 & 64 & 3.6 \\
\hline 7 & 128 & 12.5 \\
\hline 11 & 170 & 31.7 \\
\hline 15 & 256 & 85 \\
\hline 21 & 340 & 262 \\
\hline 25 & 500 & 565 \\
\hline 31 & 560 & 1585 \\
\hline
\end{tabular}

Table 3.2.2 - Simulation performance, Coated model

Adding additional circumferential modes to the simulation increases solve times considerably, though the number of digits need not increase. Solving the base model with $n_{\text {pts }}=7$ requires nearly $\mathbf{7}$ hours $(25,000 \mathrm{sec})$ per frequency. While this project is a good candidate for parallel processing, the project sponsor desires that the simulation code is able to run on an isolated machine with a single processor core. However, parallelization will be discussed in future work. 


\section{Chapter 4}

\section{Advanced Features and Validation}

With the base model validated and its behavior studied, the more advanced models are developed and each one is validated with an appropriate reference. The infinitesimally-thin delta connectors of the base system are modified to be finite-length step connectors, which transmits force to and from the shell over a finite area. Linear spring rib models have been used for Model 1 and 2; Model 3 derives the curved Timoshenko-beam based rib system and implements them with the standard elastic shell. After validation, the model is submerged in an infinite pool of acoustic fluid, which strongly affects the response of the system. Finally, in section 4.4, the acoustic coating is applied to the reinforced elastic shell and is validated and the convergence checked. This final model incorporates all the prior changes from Chapter 4.

\subsection{Finite-Width Ribs}

\subsubsection{Step Rib Validation}

Finite rib widths are discussed in Chapter 2.2, and were incorporated in the thin-shell model of Chapter 2.3. Making use of Step connectors (Heaviside functions) means that the rib forces are transmitted into the shell across the entire rib width, $b$, which is roughly 1 inch or $0.025 \mathrm{~m}$. The connectors are independent of the rib model (spring, beam) and the shell model (thin, elastic), so there are no other changes required to assess the elastic model results. Convergence has been achieved with 31 axial and 7 circumferential modes, as shown in Figures 3.1.23 and 3.1.24 from the Base Results Chapter. 

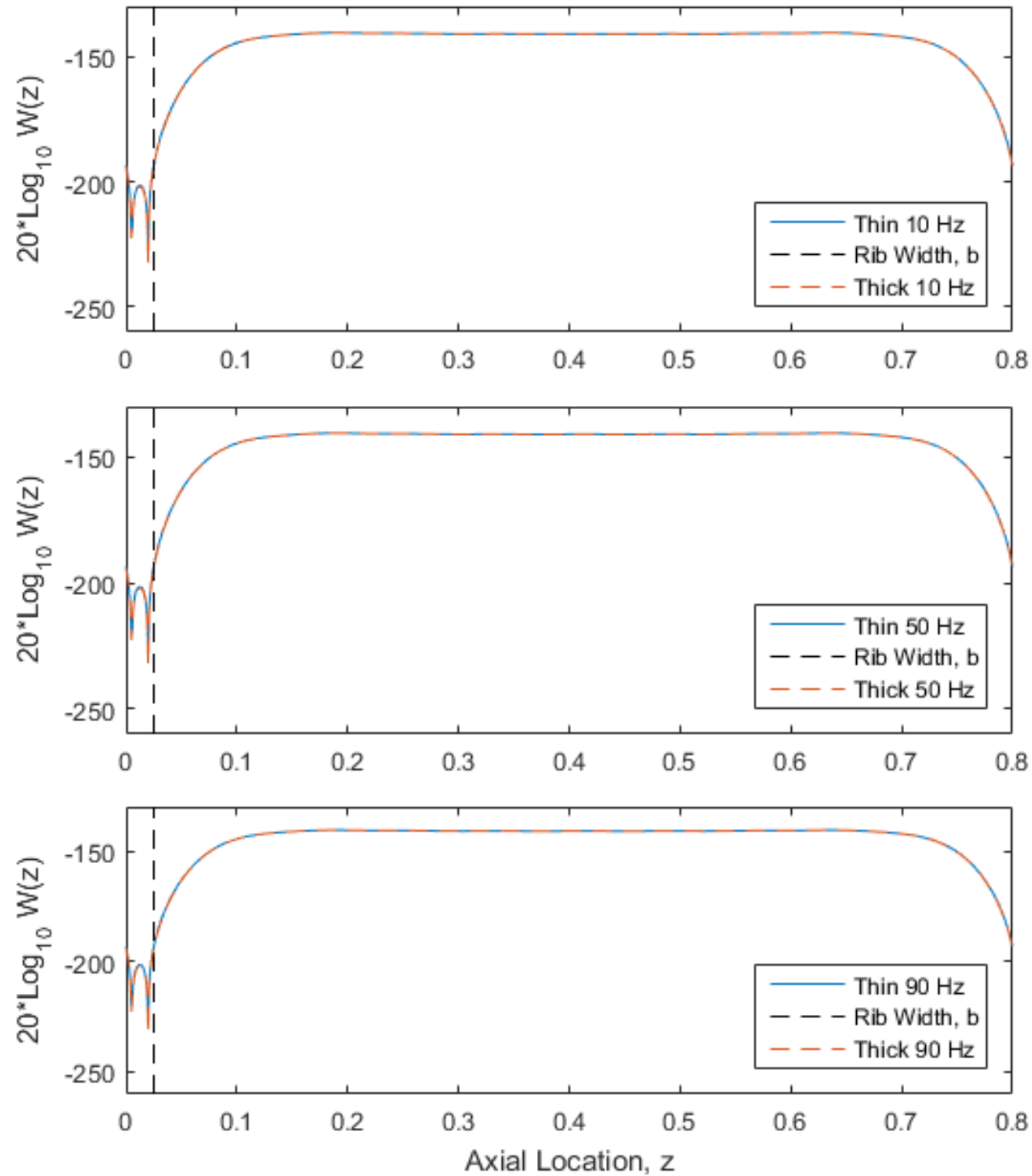

Figure 4.1.1 - Comparison of reference $\left(m_{p t s}=31\right)$ and elastic $\left(m_{p t s}=31\right)$ radial displacement magnitude for ring load $(\mathrm{k}=0)$ 

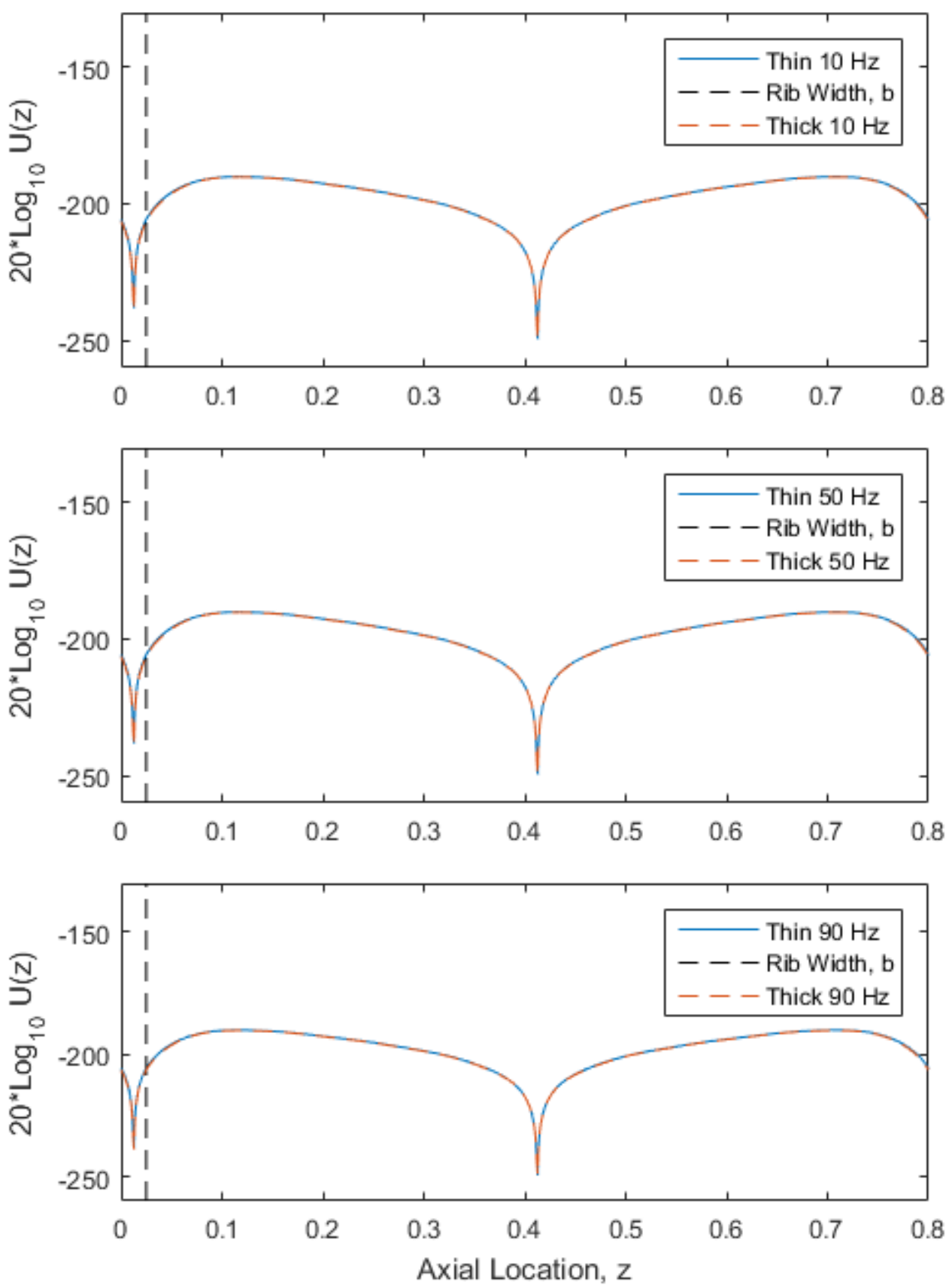

Figure 4.1.2 - Comparison of reference $\left(\mathrm{m}_{\mathrm{pts}}=31\right)$ and elastic $\left(\mathrm{m}_{\mathrm{pts}}=31\right)$ axial displacement magnitude for ring load $(\mathrm{k}=0)$

Using the same number of truncated terms $\left(m_{p t s}=31\right)$, the elastic and reference model match extremely well. The finite-length rib is located between $\mathrm{z}=0$ and $\mathrm{z}=\mathrm{b}$, denoted on the plots by the vertical dashed black line. Displacement responses are symmetric within the rib width, $0 \leq \mathrm{z} \leq \mathrm{b}$, and also in the space between the ribs, $\mathrm{b} \leq \mathrm{z} \leq \mathrm{L}$, where the next rib (and next section) starts. Since the displacement response from $0 \leq \mathrm{z} \leq \mathrm{L}$ is period, the values at the endpoints must be continuous to ensure continuity. 
Inputting an acoustic wave into the base model with finite-ribs excites the shell in a more complex manner; tangential motion is present and nonzero circumferential modes are required to describe the behavior. Following the method of Chapter 3.1, the normalized error between the thin and thick models is calculated according to Equation 3.1.8.
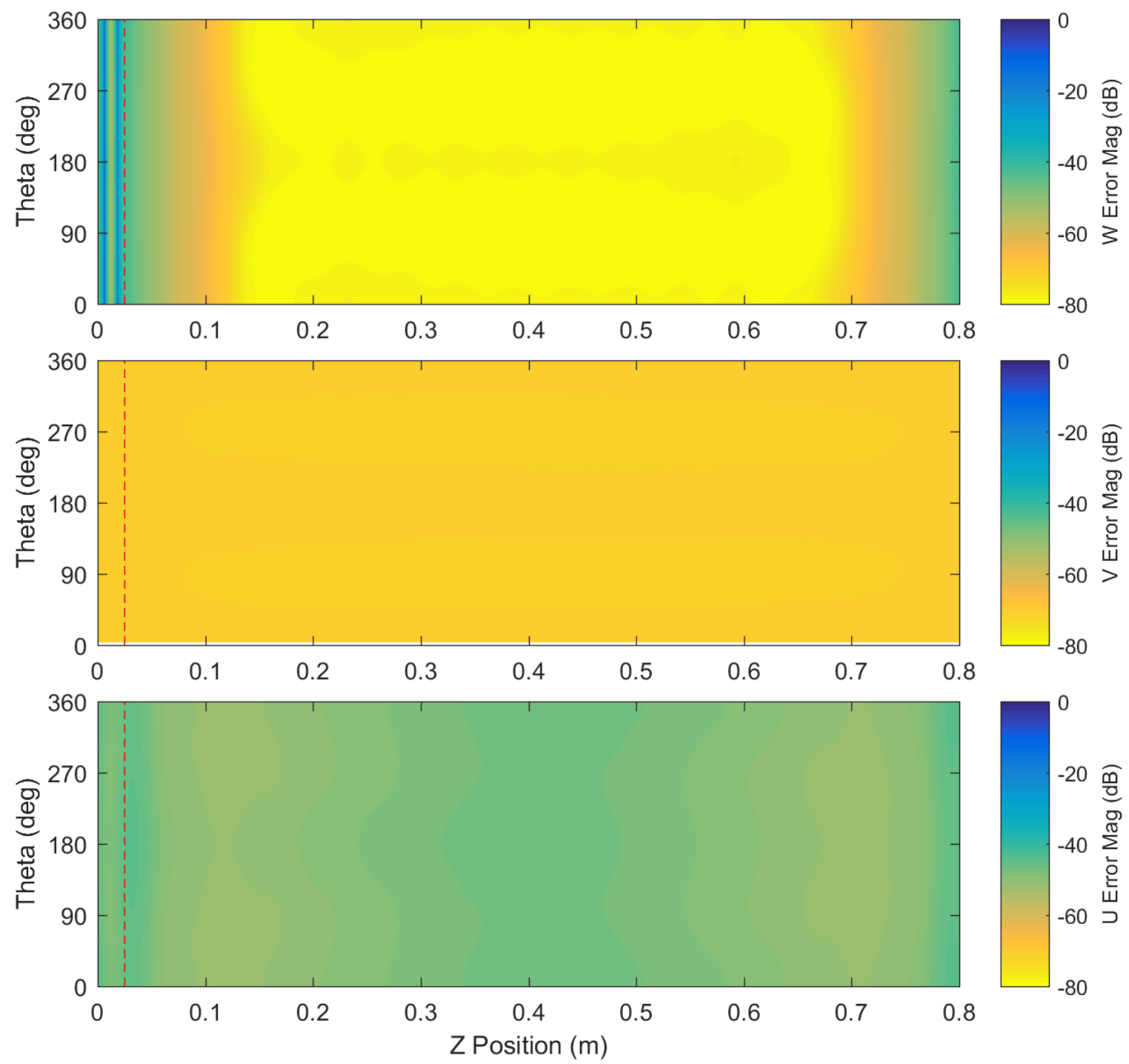

Figure 4.1.3 - Thin and Thick-shell radial (top), tangential (mid), and axial (bottom) Normalized Error in $\mathrm{dB}$ for plane-wave: $50 \mathrm{~Hz}, \varphi \mathrm{i}=\pi / 12$, mpts $=31$, npts $=7$. Rib is located between the left edge $(\mathrm{z}=0)$ and the red dashed line.

Once again, the axial response contains the largest error, however the maximum magnitude is $47 \mathrm{~dB}$ representing roughly $0.5 \%$ error. Within the rib region $(0 \leq z \leq 0.025)$ of the radial response, the error does grow to $6 \%$, but the magnitude of this region drops significantly $(-80 \mathrm{~dB})$ relative to the rest of the shell element displacement. 


\subsection{Timoshenko Beam Rib Model}

\subsubsection{Integrating Beam Model}

One of the major limitations of the base model is the accuracy of the linear spring rib model at higher frequencies. Improving the bandwidth of the stiffeners allows the total system to be excited over a much larger range, and thus a Timoshenko beam model is introduced. The dynamic beam equations were derived and decoupled to remove the angular beam deflection variable in Chapter 2.2. This puts them in a format compatible with the rest of the model, but with a significant increase in complexity compared to the spring model. Finite-length (step) connectors discussed previously will be implemented to present the most general case. Starting with the new rib equations $(2.2 .27-2.2 .29)$ provides the stiffener forces in the radial direction as

$$
\begin{gathered}
\frac{1}{\mathrm{~K}_{\mathrm{s}} \mathrm{Ga}_{\mathrm{r}}^{3}}\left(\mathrm{IE}^{2} \frac{\partial^{3} \mathrm{v}}{\partial \theta^{3}}-\rho \mathrm{EIa}_{\mathrm{r}}^{2} \frac{\partial^{3} \mathrm{v}}{\partial \theta \partial \mathrm{t}^{2}}+\mathrm{K}_{\mathrm{s}} \mathrm{GIE}^{2} \frac{\partial^{3} \mathrm{v}}{\partial \theta^{3}}-\mathrm{K}_{\mathrm{s}} \mathrm{GI}_{\rho} \mathrm{a}_{\mathrm{r}} \frac{\partial^{3} \mathrm{v}}{\partial \theta \partial \mathrm{t}^{2}}+\mathrm{K}_{\mathrm{s}} \mathrm{GAEa}_{\mathrm{r}}^{2} \frac{\partial \mathrm{v}}{\partial \theta}+\mathrm{IE}^{2} \frac{\partial^{2} \mathrm{w}}{\partial \theta^{2}}\right. \\
-\rho \mathrm{EIa}_{\mathrm{r}}^{2} \frac{\partial^{4} \mathrm{w}}{\partial \theta^{2} \partial \mathrm{t}^{2}}-\rho \mathrm{EIa}_{\mathrm{r}}^{2} \frac{\partial^{2} \mathrm{w}}{\partial \mathrm{t}^{2}}+\mathrm{K}_{\mathrm{s}} \mathrm{GIE} \frac{\partial^{4} \mathrm{w}}{\partial \theta^{4}}+\rho^{2} \mathrm{Ia}_{\mathrm{r}}^{4} \frac{\partial^{4} \mathrm{w}}{\partial \mathrm{t}^{4}}-\rho \mathrm{K}_{\mathrm{s}} \mathrm{GAa}_{\mathrm{r}}^{4} \frac{\partial^{2} \mathrm{w}}{\partial \mathrm{t}^{2}} \\
\left.-\mathrm{K}_{\mathrm{s}} \mathrm{GIa}_{\mathrm{r}}^{2} \rho \frac{\partial^{4} \mathrm{w}}{\partial \theta^{2} \partial \mathrm{t}^{2}}+\mathrm{K}_{\mathrm{s}} \mathrm{GAEa}_{\mathrm{r}}^{2} \mathrm{w}\right)=\mathrm{f}_{\mathrm{r}}
\end{gathered}
$$

in the tangential direction as

$$
\begin{aligned}
\left(\frac{E A}{a_{r}} \frac{\partial v^{2}}{\partial \theta^{2}}-\rho A\right. & \left.a_{r} \frac{\partial^{2} v}{\partial t^{2}}-\frac{\rho I}{a_{r}} \frac{\partial^{2} v}{\partial t^{2}}+\frac{E I}{a_{r}^{3}} \frac{\partial^{2} v}{\partial \theta^{2}}+\frac{\rho^{2} I a_{r}}{K_{s} G} \frac{\partial^{4} v}{\partial t^{4}}+\frac{E^{2} I}{K_{s} G a_{r}^{3}} \frac{\partial^{4} v}{\partial \theta^{4}}-\frac{2 E I \rho}{K_{s} G a_{r}} \frac{\partial^{4} v}{\partial \theta^{2} \partial t^{2}}\right) \\
& +\frac{1}{K_{s} G a_{r}^{3}}\left(I E^{2} \frac{\partial^{3} w}{\partial \theta^{3}}-\rho E I a_{r}^{2} \frac{\partial^{3} w}{\partial \theta \partial t^{2}}+K_{s} G I E \frac{\partial^{3} w}{\partial \theta^{3}}-K_{s} G I \rho a_{r}^{2} \frac{\partial^{3} w}{\partial \theta \partial t^{2}}\right. \\
& \left.+K_{s} G A E a_{r}^{2} \frac{\partial w}{\partial \theta}\right)=f_{\theta}
\end{aligned}
$$

and in the axial direction as

$$
-\frac{2 G J}{h a_{r}^{2}} \frac{\partial^{3} w}{\partial \theta^{2} \partial z}-\frac{2 \rho J}{h} \frac{\partial^{2} w}{\partial t^{2} \partial z}=f_{z}
$$

Recall that the axial stiffener force 4.2.3 is independent from the circular beam 4.2.1 - 4.2.2. To simplify the equations, the following effective stiffness terms are defined, taking advantage of the harmonic forms of the displacements 


$$
\begin{gathered}
K_{r w}=\frac{1}{K_{s} G a_{r}^{3}}\left(-I E^{2} n^{2}-\rho E I a_{r}^{2} n^{2} \omega^{2}+\rho E I a_{r}^{2} \omega^{2}+K_{s} G I E n^{4}\right. \\
\left.+\rho^{2} I a_{r}^{4} \omega^{4}+\rho K_{s} G A a_{r}^{4} \omega^{2}-K_{s} G I a_{r}^{2} \rho n^{2} \omega^{2}+K_{s} G A E a_{r}^{2}\right) \\
K_{r v}=\frac{1}{K_{S} G a_{r}^{3}}\left(-I E^{2} n^{3}+\rho E I a_{r}^{2} n \omega^{2}-K_{s} G I E^{2} n^{3}+K_{s} G I \rho a_{r} n \omega^{2}-K_{s} G A E a_{r}^{2} n^{2}\right) \\
K_{\theta w}=\frac{1}{K_{S} G a_{r}^{3}}\left(I E^{2} n^{3}+\rho E I a_{r}^{2} n \omega^{2}+K_{s} G I E n^{3}+K_{s} G I \rho a_{r}^{2} n \omega^{2}-K_{s} G A E a_{r}^{2} n\right) \\
K_{\theta v}=-\frac{E A}{a_{r}} n^{2}+\rho A a_{r} \omega^{2}+\frac{\rho I}{a_{r}} \omega^{2}-\frac{E I}{a_{r}^{3}} n^{2}+\frac{\rho^{2} I a_{r}}{K_{s} G} \omega^{4}+\frac{E^{2} I}{K_{s} G a_{r}^{3}} n^{4}-\frac{2 E I \rho}{K_{s} G a_{r}} n^{2} \omega^{2} \\
K_{z w}=\frac{2 G J}{h a_{r}^{2}} k_{m} n^{2}+\frac{2 \rho J}{h} k_{m} \omega^{2}
\end{gathered}
$$

Note that the stiffness terms are dependent on circumferential index, $\mathrm{n}$. To integrate the above rib equations with the shell, they can be inserted into the inner surface boundary conditions, Equations 2.4.12-2.4.14.

$$
\begin{aligned}
& \sum_{m=-\infty}^{\infty} \sum_{n=0}^{\infty}\left\{(\lambda+2 \mu) \frac{d W_{m n}(a)}{d r}+\frac{\lambda}{a} W_{m n}(a)+\frac{\lambda n}{a} V_{m n}(a)+\lambda i k_{m} U_{m n}(a)\right\} \cos (n \theta) e^{-i k_{m} z} \\
&= \frac{1}{a b} \sum_{m=-\infty}^{\infty} \sum_{n=0}^{\infty}\left\{K_{r w} W_{m n}+K_{r v} V_{m n}\right\} \cos (n \theta) e^{-i k_{m} z} \sum_{s=-\infty}^{\infty}[H(z-s L)-H(z-b-s L)] \\
&=\frac{1}{a b} \sum_{m=-\infty} \sum_{n=0}^{\infty}\left\{K_{\theta w} W_{m n}+K_{\theta v} V_{m n}\right\} \sin (n \theta) e^{-i k_{m} z} \sum_{s=-\infty}^{\infty}[H(z-s L)-H(z-b-s L)] \\
& \quad \sum_{n=-\infty}^{\infty}\left\{\mu \frac{d V_{m n}(a)}{d r}-\frac{\mu}{a} V_{m n}(a)-\frac{\mu n}{a} W_{m n}(a)\right\} \sin (n \theta) e^{-i k_{m} z} \\
&=\frac{1}{a b} \sum_{m=-\infty}^{\infty} \sum_{n=0}^{\infty}\left\{K_{z w} W_{m n}\right\} \cos (q \theta) e^{i k_{m} z} \sum_{s=-\infty}^{\infty}[H(z-s L)-H(z-b-S L)]
\end{aligned}
$$

With the Timoshenko beam rib model integrated into the inner surface $(r=a)$ boundary conditions, the procedure described in Chapter 2.3 and 2.4 can be followed. Step functions are converted into their Fourier series, and the index shift (Equation 2.3.16) is applied. Combining the inner boundary equations with the unchanged outer boundary equations (2.4.21 - 2.4.23). Finally, the orthogonalization process is performed. 


\subsubsection{Decoupled Form and Global Matrix Equation}

Multiplying by $\mathrm{e}^{-\mathrm{i} \mathrm{k}_{\mathrm{m} 2} \mathrm{z}} \cos \left(\mathrm{n}_{2} \theta\right)$ and integrating from $[0,2 \pi]$ on $\mathrm{n}$ and $[0, \mathrm{~L}]$ on $\mathrm{z}$ results in the 6 stress equations, decomposed into an infinite set of $(m, n)$-indexed equations with the harmonic terms eliminated. At the inner boundary, the radial-normal and radial shear equations now include the beam model

$$
\begin{gathered}
(\lambda+2 \mu) \frac{d W_{m n}(a)}{d r}+\frac{\lambda}{a} W_{m n}(a)+\frac{\lambda n}{a} V_{m n}(a)+\lambda i k_{m} U_{m n}(a) \\
=\frac{1}{a b} \sum_{s=-\infty}^{\infty}\left\{K_{r w} W_{s n}+K_{r v} V_{s n}\right\} d_{m-s} \\
\mu \frac{d V_{m n}(a)}{d r}-\frac{\mu}{a} V_{m n}(a)-\frac{\mu n}{a} W_{m n}(a)=\frac{1}{a b} \sum_{s=-\infty}^{\infty}\left\{K_{\theta w} W_{s n}+K_{\theta v} V_{s n}\right\} d_{m-s} \\
\mu i k_{m} W_{m n}(a)+\mu \frac{d U_{m n}(a)}{d r}=\frac{1}{a b} \sum_{s=-\infty}^{\infty} K_{z w} W_{s n} d_{m-s}
\end{gathered}
$$

with $d_{m-s}$ resulting from the Step connector Fourier series, described in Chapter 2.2. At the outer boundary, 2.4.30 - 2.4.32 remain unchanged. Displacement coefficients $\left(W_{m n}, V_{m n}, U_{m n}\right)$ must now be replaced by their unknown propagation coefficients $\left(A_{m n}, B_{m n}, C_{m n}, D_{m n}, E_{m n}, F_{m n}\right)$, as specified in Equations 2.4.6 - 2.4.8. Only the first 3 equations of $2.4 .33-2.4 .38$ have been modified and are 


$$
\begin{gathered}
{\left[\frac{2 \alpha_{m} \mu}{a} J_{n+1}\left(\alpha_{m} a\right)+\left(\frac{2 n(n-1) \mu}{a^{2}}-\alpha_{m}^{2} l \lambda-2 \alpha_{m}^{2} \mu-k_{z m}^{2} \lambda\right) J_{n}\left(\alpha_{m} a\right)\right] A_{m n}} \\
+\left[\frac{2 \alpha_{m} \mu}{a} Y_{n+1}\left(\alpha_{m} a\right)+\left(\frac{2 n(n-1) \mu}{a^{2}}-\alpha_{m}^{2} l \lambda-2 \alpha_{m}^{2} \mu-k_{z m}^{2} \lambda\right) Y_{n}\left(\alpha_{m} a\right)\right] B_{m n} \\
+\left[-\frac{2 n \beta_{m} \mu}{a} J_{n+1}\left(\beta_{m} a\right)+\frac{2 n(n-1) \mu}{a^{2}} J_{n}\left(\beta_{m} a\right)\right] C_{m n} \\
+\left[-\frac{2 n \beta_{m} \mu}{a} Y_{n+1}\left(\beta_{m} a\right)+\frac{2 n(n-1) \mu}{a^{2}} Y_{n}\left(\beta_{m} a\right)\right] D_{m n} \\
+\left[-\frac{2 n(n+1) i k_{m} \mu}{a} J_{n+1}\left(\beta_{m} a\right)+2 i k_{m} \beta_{m} \mu J_{n}\left(\beta_{m} a\right)\right] E_{m n} \\
+\left[-\frac{2 n(n+1) i k_{m} \mu}{a} Y_{n+1}\left(\beta_{m} a\right)+2 i k_{m} \beta_{m} \mu Y_{n}\left(\beta_{m} a\right)\right] F_{m n} \\
=\frac{1}{a b} \sum_{s=-\infty}^{\infty} K_{r w}\left\{\left[-\alpha_{s} J_{n+1}\left(\alpha_{s} a\right)+\frac{n}{r} J_{n}\left(\alpha_{s} a\right)\right] A_{s n}+\left[-\alpha_{s} Y_{n+1}\left(\alpha_{s} a\right)+\frac{n}{a} Y_{n}\left(\alpha_{s} a\right)\right] B_{s n}\right. \\
\left.+\frac{n}{a} J_{n}\left(\beta_{s} a\right) C_{s n}+\frac{n}{a} Y_{n}\left(\beta_{s} a\right) D_{s n}+i k_{s} J_{n+1}\left(\beta_{s} a\right) E_{s n}+i k_{s} Y_{n+1}\left(\beta_{s} a\right) F_{s n}\right\} d_{m-s} \\
+K_{r v}\left\{-\frac{n}{a} J_{n}\left(\alpha_{s} a\right) A_{s n}-\frac{n}{a} Y_{n}\left(\alpha_{s} a\right) B_{s n}+\left[\beta_{s} J_{n+1}\left(\beta_{s} a\right)-\frac{n}{a} J_{n}\left(\beta_{s} a\right)\right] C_{s n}\right. \\
\left.+\left[\beta_{s} Y_{n+1}\left(\beta_{s} a\right)-\frac{n}{a} Y_{n}\left(\beta_{s} a\right)\right] D_{s n}+i k_{s} J_{n+1}\left(\beta_{s} a\right) E_{s n}+i k_{s} Y_{n+1}\left(\beta_{s} a\right) F_{s n}\right\} d_{m-s}
\end{gathered}
$$




$$
\begin{aligned}
& {\left[\frac{2 n \alpha_{m} \mu}{a} J_{n+1}\left(\alpha_{m} a\right)-\frac{2 n(n-1) \mu}{a^{2}} J_{n}\left(\alpha_{m} a\right)\right] A_{m n}} \\
& +\left[\frac{2 n \alpha_{m} \mu}{a} Y_{n+1}\left(\alpha_{m} a\right)-\frac{2 n(n-1) \mu}{a^{2}} Y_{n}\left(\alpha_{m} a\right)\right] B_{m n} \\
& +\left[-\frac{\beta_{m} \mu}{a} J_{n+1}\left(\beta_{m} a\right)+\left(\beta_{m}^{2} \mu-\frac{2 n(n-1) \mu}{a^{2}}\right) J_{n}\left(\beta_{m} a\right)\right] C_{m n} \\
& +\left[-\frac{\beta_{m} \mu}{a} Y_{n+1}\left(\beta_{m} a\right)+\left(\beta_{m}^{2} \mu-\frac{2 n(n-1) \mu}{a^{2}}\right) Y_{n}\left(\beta_{m} a\right)\right] D_{m n} \\
& +\left[-\frac{2(n+1) i k_{m} \mu}{a} J_{n+1}\left(\beta_{m} a\right)+i k_{m} \beta_{m} \mu J_{n}\left(\beta_{m} a\right)\right] E_{m n} \\
& +\left[-\frac{2(n+1) i k_{m} \mu}{a} Y_{n+1}\left(\beta_{m} a\right)+i k_{m} \beta_{m} \mu Y_{n}\left(\beta_{m} a\right)\right] F_{m n} \\
& =\frac{1}{a b} \sum_{s=-\infty}^{\infty} K_{\theta v}\left\{-\frac{n}{a} J_{n}\left(\alpha_{s} a\right) A_{s n}-\frac{n}{a} Y_{n}\left(\alpha_{s} a\right) B_{s n}+\left[\beta_{s} J_{n+1}\left(\beta_{s} a\right)-\frac{n}{a} J_{n}\left(\beta_{s} a\right)\right] C_{s n}\right. \\
& \left.+\left[\beta_{s} Y_{n+1}\left(\beta_{s} a\right)-\frac{n}{a} Y_{n}\left(\beta_{s} a\right)\right] D_{s n}+i k_{s} J_{n+1}\left(\beta_{s} a\right) E_{s n}+i k_{s} Y_{n+1}\left(\beta_{s} a\right) F_{s n}\right\} d_{m-s} \\
& K_{r w}\left\{\left[-\alpha_{s} J_{n+1}\left(\alpha_{s} a\right)+\frac{n}{r} J_{n}\left(\alpha_{s} a\right)\right] A_{s n}+\left[-\alpha_{s} Y_{n+1}\left(\alpha_{s} a\right)+\frac{n}{a} Y_{n}\left(\alpha_{s} a\right)\right] B_{s n}\right. \\
& \left.+\frac{n}{a} J_{n}\left(\beta_{s} a\right) C_{s n}+\frac{n}{a} Y_{n}\left(\beta_{s} a\right) D_{s n}+i k_{s} J_{n+1}\left(\beta_{s} a\right) E_{s n}+i k_{s} Y_{n+1}\left(\beta_{s} a\right) F_{s n}\right\} d_{m-s} \\
& {\left[-2 i k_{m} \alpha_{m} J_{n+1}\left(\alpha_{m} a\right)+\frac{2 n i k_{m} \mu}{a} J_{n}\left(\alpha_{m} a\right)\right] A_{m n}} \\
& +\left[-2 i k_{m} \alpha_{m} Y_{n+1}\left(\alpha_{m} a\right)+\frac{2 n i k_{m} \mu}{a} Y_{n}\left(\alpha_{m} a\right)\right] B_{m n} \\
& +\left[\frac{n i k_{m} \mu}{a} J_{n}\left(\beta_{m} a\right)\right] C_{m n}+\left[\frac{n i k_{m} \mu}{a} Y_{n}\left(\beta_{m} a\right)\right] D_{m n} \\
& +\left[\mu\left(\beta_{m}^{2}-k_{m}^{2}\right) J_{n+1}\left(\beta_{m} a\right)-\frac{n \beta_{m} \mu}{a} J_{n}\left(\beta_{m} a\right)\right] E_{m n} \\
& +\left[\mu\left(\beta_{m}^{2}-k_{m}^{2}\right) Y_{n+1}\left(\beta_{m} a\right)-\frac{n \beta_{m} \mu}{a} Y_{n}\left(\beta_{m} a\right)\right] F_{m n} \\
& =\frac{1}{a b} \sum_{s=-\infty}^{\infty} K_{r w}\left\{\left[-\alpha_{s} J_{n+1}\left(\alpha_{s} a\right)+\frac{n}{r} J_{n}\left(\alpha_{s} a\right)\right] A_{s n}+\left[-\alpha_{s} Y_{n+1}\left(\alpha_{s} a\right)+\frac{n}{a} Y_{n}\left(\alpha_{s} a\right)\right] B_{s n}\right. \\
& \left.+\frac{n}{a} J_{n}\left(\beta_{s} a\right) C_{s n}+\frac{n}{a} Y_{n}\left(\beta_{s} a\right) D_{s n}+i k_{s} J_{n+1}\left(\beta_{s} a\right) E_{s n}+i k_{s} Y_{n+1}\left(\beta_{s} a\right) F_{s n}\right\} d_{m-s}
\end{aligned}
$$

Implementing the new beam rib model has no effect on the shell dynamics, and thus the system $\mathbf{A}_{\mathrm{mn}}$ matrix is unaffected compared to Chapter 2.4. In fact, only the elements of the stiffener matrices $\left(\mathbf{R}_{\mathbf{m}-\mathbf{s}}, \mathbf{T}_{\mathbf{m}-\mathbf{s}}, \mathbf{Z}_{\mathbf{m}-\mathrm{s}}\right)$ are different. Each are $6 \times 6$, and contain only a single non-zero row shown below 


$$
\begin{aligned}
\boldsymbol{R}_{\boldsymbol{m}-s}(1,1: 6)= & d_{m-s}\left\{K _ { r w } \left[\left(-\alpha_{s} J_{n+1}\left(\alpha_{s} a\right)+\frac{n}{r} J_{n}\left(\alpha_{s} a\right)\right), \quad\left(-\alpha_{s} Y_{n+1}\left(\alpha_{s} a\right)+\frac{n}{r} Y_{n}\left(\alpha_{s} a\right)\right), \ldots\right.\right. \\
& \left.\frac{n}{a} J_{n}\left(\beta_{s} a\right), \quad i \frac{n}{a} Y_{n}\left(\beta_{s} a\right), \quad i k_{s} J_{n+1}\left(\beta_{s} a\right), \quad i k_{s} Y_{n+1}\left(\beta_{s} a\right)\right] \\
+ & K_{r v}\left[-\frac{n}{a} J_{n}\left(\alpha_{s} a\right), \quad-\frac{n}{a} Y_{n}\left(\alpha_{s} a\right), \quad\left(\beta_{s} J_{n+1}\left(\beta_{s} a\right)-\frac{n}{a} J_{n}\left(\beta_{s} a\right)\right), \ldots\right. \\
& \left.\left.\left(\beta_{s} Y_{n+1}\left(\beta_{s} a\right)-\frac{n}{a} Y_{n}\left(\beta_{s} a\right)\right), \quad i k_{s} J_{n+1}\left(\beta_{s} a\right), \quad i k_{s} J_{n+1}\left(\beta_{s} a\right)\right]\right\}
\end{aligned}
$$

$$
\begin{aligned}
\boldsymbol{T}_{\boldsymbol{m}-\boldsymbol{s}}(2,1: 6)= & d_{m-s}\left\{K _ { \theta w } \left[\left(-\alpha_{s} J_{n+1}\left(\alpha_{s} a\right)+\frac{n}{r} J_{n}\left(\alpha_{s} a\right)\right), \quad\left(-\alpha_{s} Y_{n+1}\left(\alpha_{s} a\right)+\frac{n}{r} Y_{n}\left(\alpha_{s} a\right)\right), \ldots\right.\right. \\
& \left.\frac{n}{a} J_{n}\left(\beta_{s} a\right), \quad i \frac{n}{a} Y_{n}\left(\beta_{s} a\right), \quad i k_{s} J_{n+1}\left(\beta_{s} a\right), \quad i k_{s} Y_{n+1}\left(\beta_{s} a\right)\right] \\
& K_{\theta v}\left[-\frac{n}{a} J_{n}\left(\alpha_{s} a\right), \quad-\frac{n}{a} Y_{n}\left(\alpha_{s} a\right), \quad\left(\beta_{s} J_{n+1}\left(\beta_{s} a\right)-\frac{n}{a} J_{n}\left(\beta_{s} a\right)\right), \ldots\right. \\
& \left.\left.\left(\beta_{s} Y_{n+1}\left(\beta_{s} a\right)-\frac{n}{a} Y_{n}\left(\beta_{s} a\right)\right), \quad i k_{s} J_{n+1}\left(\beta_{s} a\right), \quad i k_{s} J_{n+1}\left(\beta_{s} a\right)\right]\right\}
\end{aligned}
$$

$$
\begin{aligned}
& \boldsymbol{Z}_{\boldsymbol{m}-\boldsymbol{s}}(3,1: 6)= d_{m-s} K_{z w}\left[\left(-\alpha_{s} J_{n+1}\left(\alpha_{s} a\right)+\frac{n}{r} J_{n}\left(\alpha_{s} a\right)\right), \quad\left(-\alpha_{s} Y_{n+1}\left(\alpha_{s} a\right)+\frac{n}{r} Y_{n}\left(\alpha_{s} a\right)\right), \ldots\right. \\
&\left.\frac{n}{a} J_{n}\left(\beta_{s} a\right), \quad i \frac{n}{a} Y_{n}\left(\beta_{s} a\right), \quad i k_{s} J_{n+1}\left(\beta_{s} a\right), \quad i k_{s} Y_{n+1}\left(\beta_{s} a\right)\right]
\end{aligned}
$$

The global matrices can be constructed in the same manner as before, only the stiffness terms (eg $\mathrm{K}_{\mathrm{rw}}$ ) have been absorbed into the stiffener matrices. Thus the global matrix equation 2.4.45 becomes

$$
\widehat{\boldsymbol{A}}_{\boldsymbol{n}} \widehat{\boldsymbol{x}}_{\boldsymbol{n}}=-\frac{1}{a b} \widehat{\boldsymbol{Z}}_{\boldsymbol{n}} \widehat{\boldsymbol{x}}_{n}-\frac{1}{a b} \widehat{\boldsymbol{T}}_{\boldsymbol{n}} \widehat{\boldsymbol{x}}_{n}-\frac{1}{a b} \widehat{\boldsymbol{R}}_{\boldsymbol{n}} \widehat{\boldsymbol{x}}_{\boldsymbol{n}}+\widehat{\boldsymbol{f}}_{\boldsymbol{n}}
$$

\subsubsection{Validation with Thin-Shell Model}

Timoshenko beam ribs can be implemented with the thin-shell model in the same manner as derived above, and this provides a good method to ensure that the model behavior is sensible. Following the convergence criteria from Base Results Chapter, 31 axial modes and 7 circumferential modes are simulated. First the ring load $(k=0)$ is applied. 

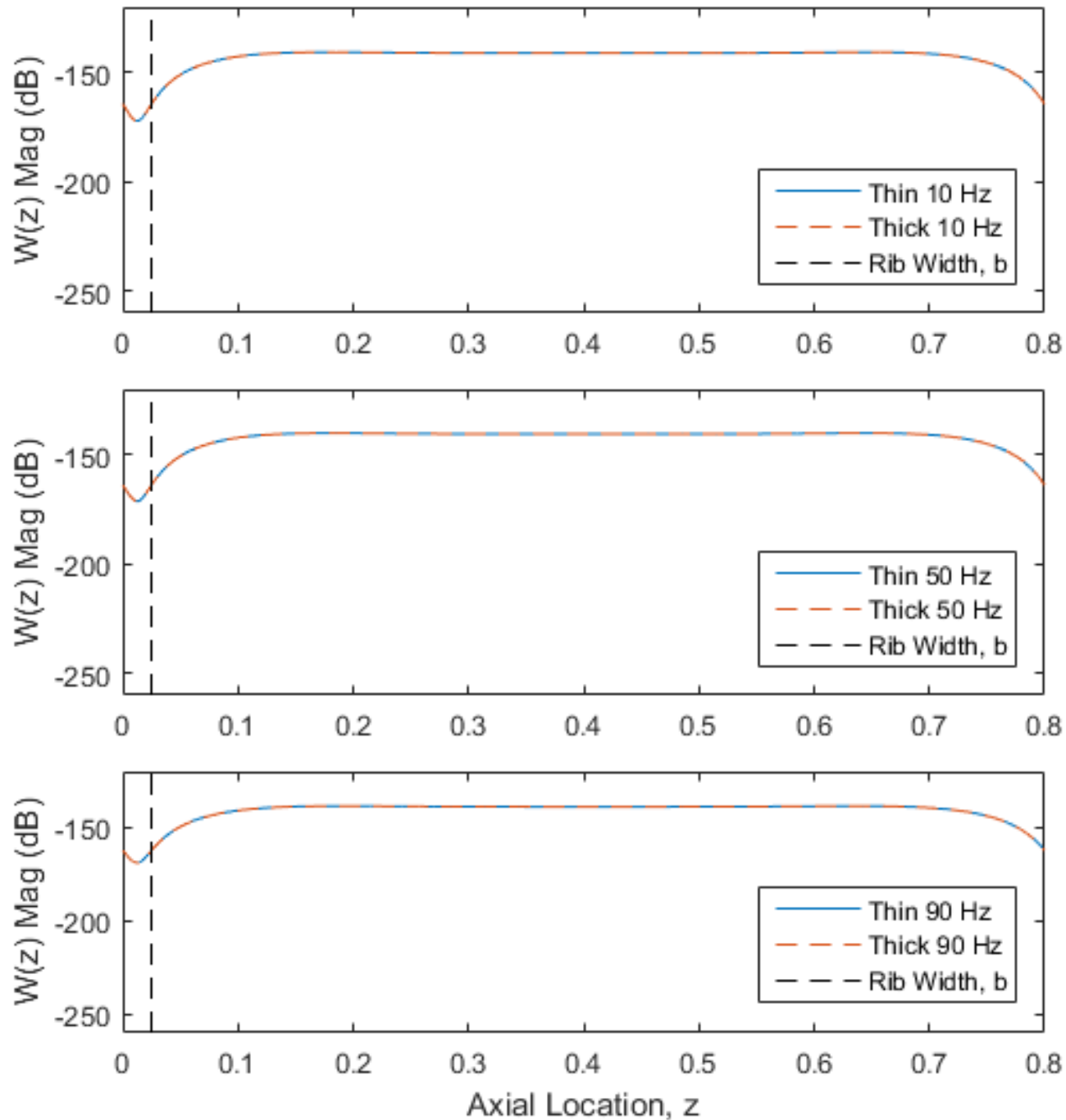

Figure 4.2.1 - Comparison of beam-rib reference and elastic radial displacement magnitude for ring load $(\mathrm{k}=0), \mathrm{m}_{\mathrm{pts}}=31$ 

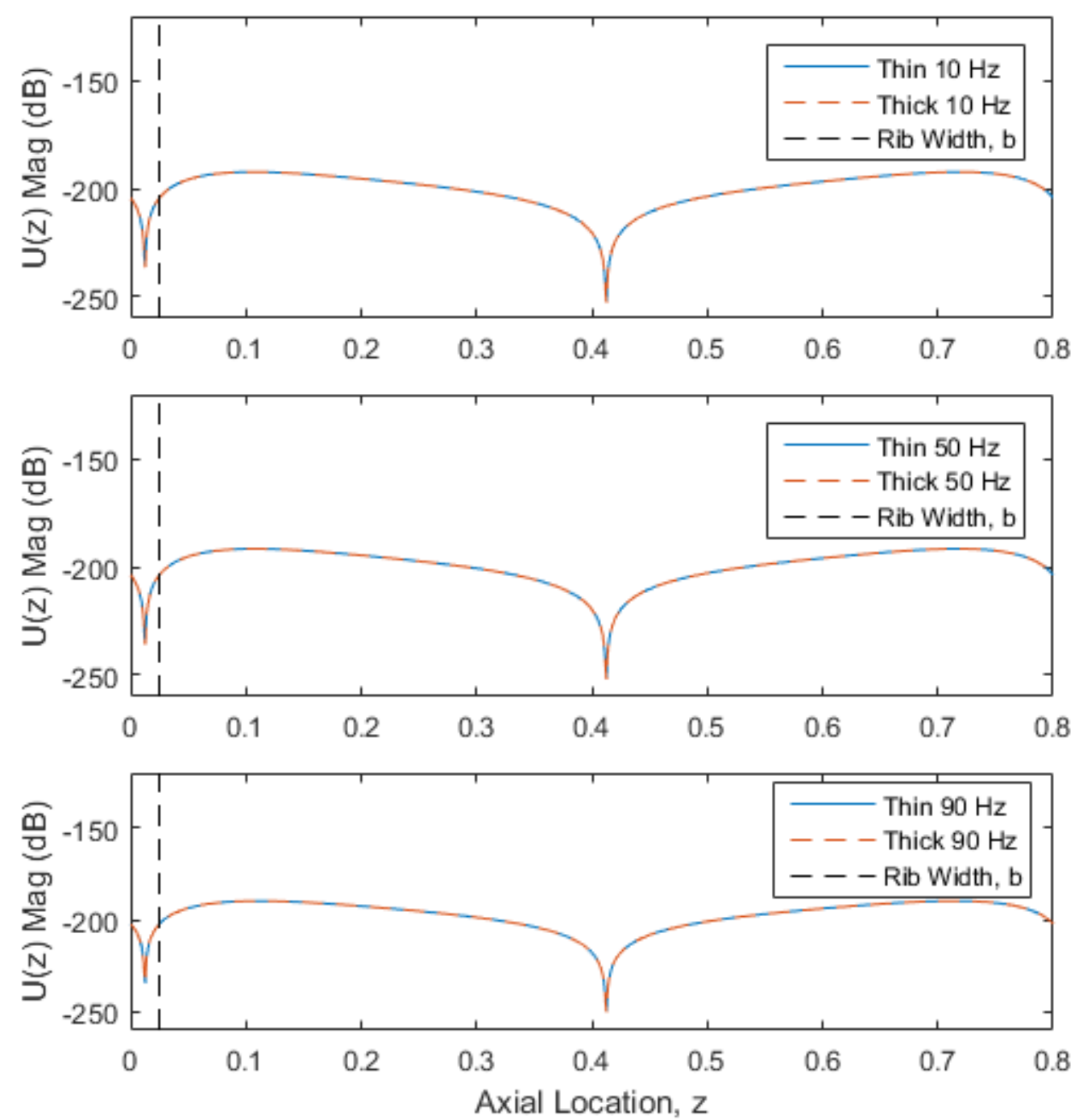

Figure 4.2.2 - Comparison of beam-rib reference and elastic axial displacement magnitude for ring load $(\mathrm{k}=0), \mathrm{m}_{\mathrm{pts}}=31$

Elastic displacement matches the reference model extremely well. Radial deformation characteristics have changed from the linear-spring rib model of Chapter 4.1, revealing that the beam ribs are less stiff than the linear-spring versions. Overall, the displacement filed is still very similar, and the largest effect of the beam rib should be in its higher frequency accuracy and the coupling of radial and tangential displacement.

The response to an acoustic wave, incident at $\theta=0$ and $\phi_{i}=15$ deg from broadside, is given in Figures 4.2.3 in terms of normalized error (Equation 3.1.8). 

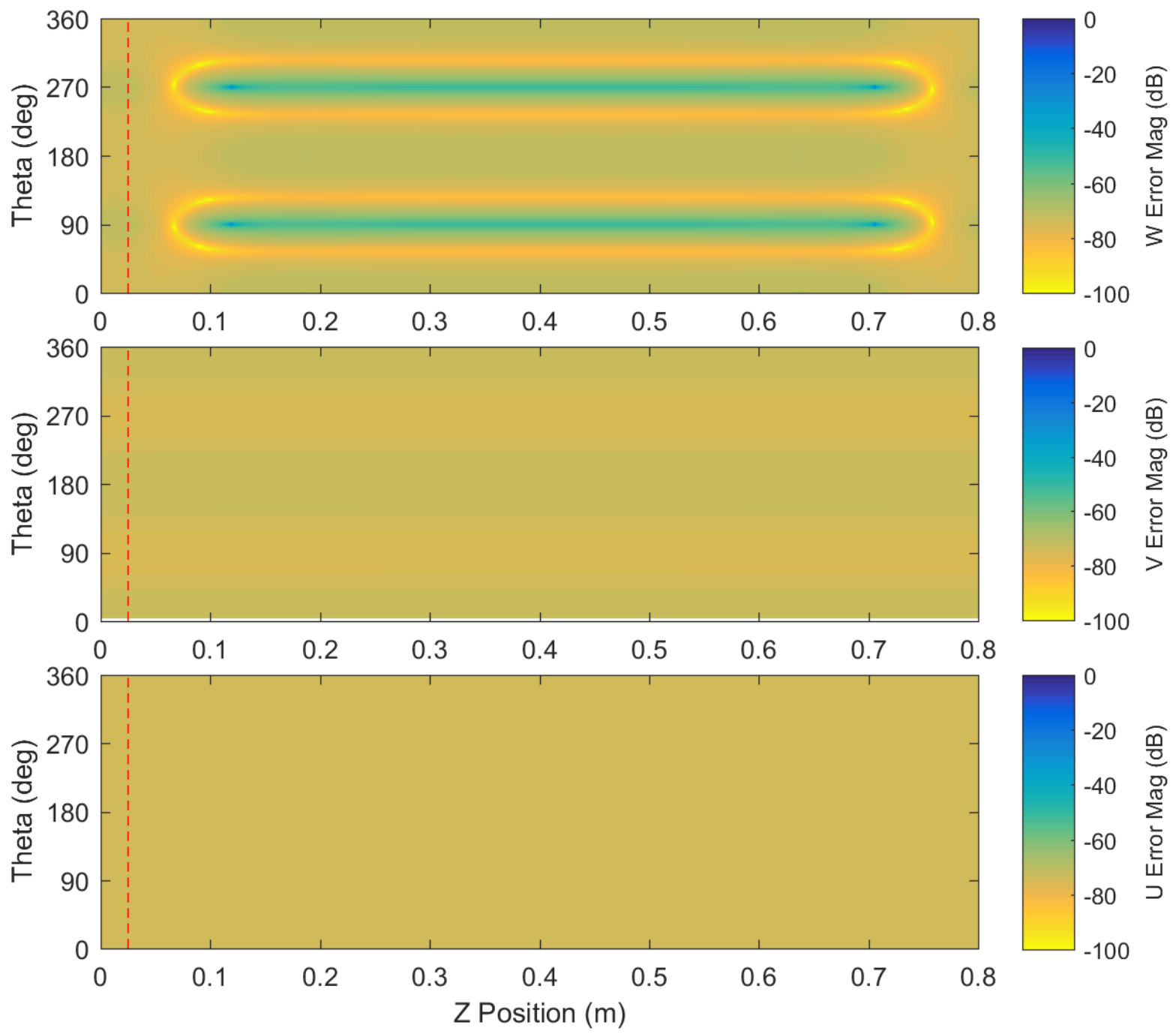

Figure 4.2.3 - Thin and Thick-shell radial (top), tangential (mid), and axial (bottom) Normalized Error in $\mathrm{dB}$ for plane-wave: $50 \mathrm{~Hz}, \varphi \mathrm{i}=\pi / 12$, mpts $=31$, npts $=7$. Rib is located between the left edge $(\mathrm{z}=0)$ and the red dashed line

Overall errors are roughly $-70 \mathrm{~dB}$ or lower, which corresponds to less than $0.05 \%$. In the upper plot of 4.2.3, there are 4 distinct locations of high error $(>50 \%)$ at $\theta=90$ and $270 \mathrm{deg}$ and $z=0.11$ and 0.71 . These locations correspond to nodes in the magnitude response, where the values drop close to zero; an example at $\theta=90 \mathrm{deg}$ is shown below. 


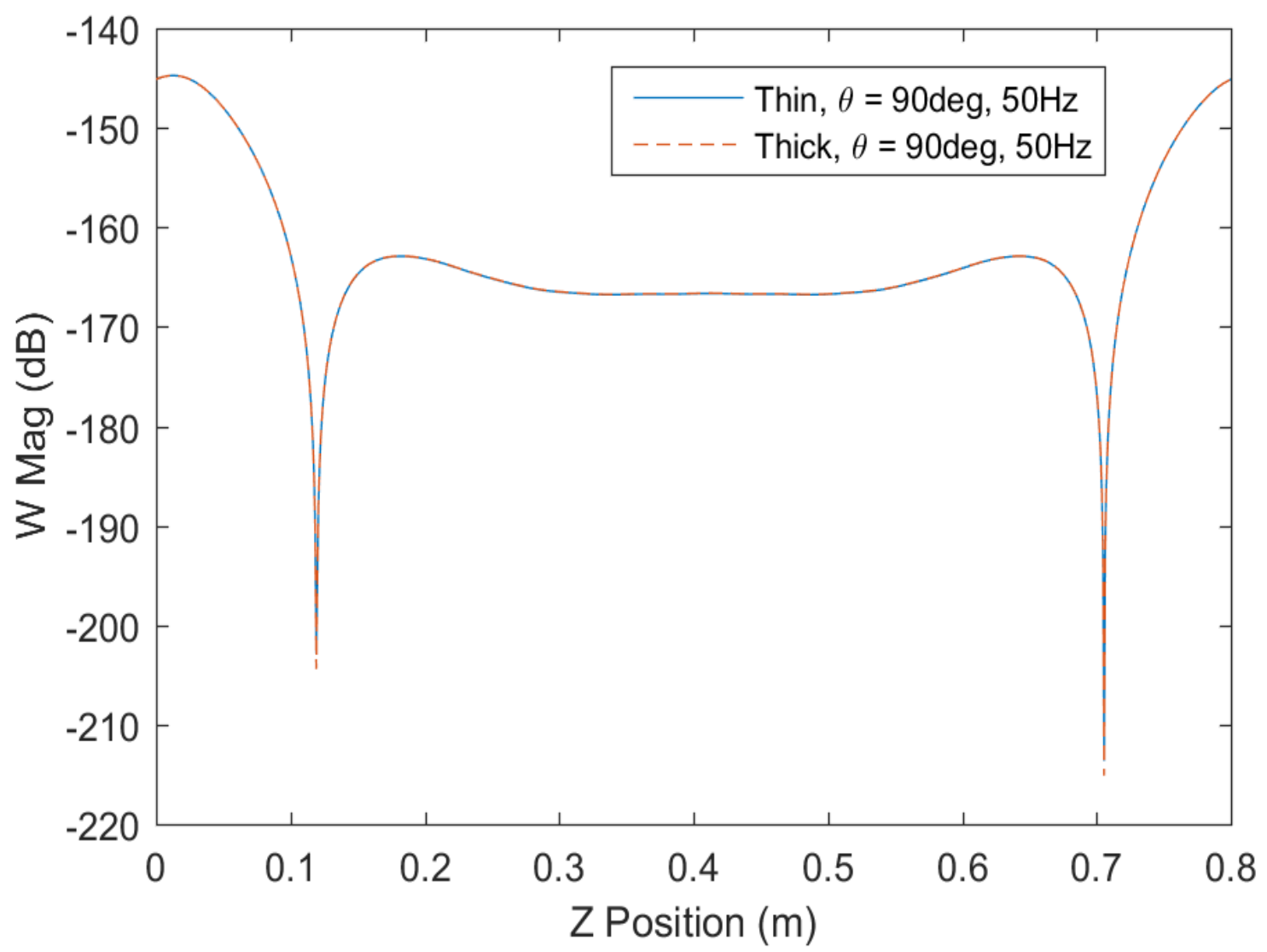

Figure 4.2.4 - Acoustic Wave radial response of reference and elastic model at $\theta=90 \mathrm{deg}$

Note that Figure 4.2.4 is showing magnitude, not relative error; it is essentially a horizontal slice through the top of Figure 4.2.3 at $\theta=90 \mathrm{deg}$. The magnitude of both the reference and elastic model drops $-40 \mathrm{~dB}$ below the remaining data at the two nodes.

\subsection{Fluid Loading}

\subsubsection{Scattered Fluid Pressure}

Fluid surrounding the shell model is assumed to extent infinitely in all directions and be lossless, such that viscous effects are neglected. Thus the inertial effect is dominant, and the fluid is treated as an acoustic medium, where shear stress cannot be transmitted. Total fluid pressure is defined as

$$
P_{a}=P_{i}+P_{f}
$$

where $P_{a}$ is the applied fluid pressure, $P_{i}$ is the incident fluid pressure exciting the system and $P_{f}$ is the scattered fluid pressure caused by the system displacements. The model excitation $P_{i}$ is a dilatational plane wave input at the outer surface $(r=c)$ of the cylinder, which is defined as 


$$
P_{i}=P_{0} e^{i k_{z} z} \sum_{n=0}^{\infty} \varepsilon_{n} i^{n} J_{n}\left(k_{r} r\right) \cos (n \theta) e^{-i \omega t}
$$

where $\varepsilon$ is the Neumann factor and $\mathrm{J}_{\mathrm{n}}$ is a $\mathrm{n}^{\text {th }}$ order Bessel function of the $1^{\text {st }}$ kind, $\mathrm{k}_{\mathrm{r}}$ and $\mathrm{k}_{\mathrm{z}}$ are the radial and longitudinal components of the input wavenumber, $k$. This wave is derived in Excitations Chapter 2.5. Fluid pressure is governed by the three-dimensional wave equation (cylindrical coordinates) expressed as

$$
\frac{\partial^{2} P_{f}}{\partial r^{2}}+\frac{1}{r} \frac{\partial P_{f}}{\partial r}+\frac{1}{r^{2}} \frac{\partial^{2} P_{f}}{\partial \theta^{2}}+\frac{\partial^{2} P_{f}}{\partial z^{2}}=\frac{1}{c_{f}^{2}} \frac{\partial^{2} P_{f}}{\partial t^{2}}
$$

where $c_{f}$ is the compression wave speed. To find the scattered wave, the equation must be solved for $P_{f}$. General solutions for the 3D wave equation (for scalars) are found via separation of variables. The procedure is outlined in the Appendix [*].

$$
P_{f}=e^{i k_{\mathrm{Z}} z} \sum_{n=0}^{\infty} R_{n} H_{n}^{1}\left(k_{r} r\right) \cos (n \theta) e^{-i \omega t}
$$

in which $R_{n}$ is the unknown coefficient, and $\mathrm{H}_{n}{ }^{1}$ is a $\mathrm{n}^{\text {th }}$ order Hankel function of the first kind. $\mathrm{k}_{\mathrm{r}}$ and $\mathrm{k}_{\mathrm{z}}$ are the constant radial and axial wavenumbers, respectively. For the reinforced system, however, radial displacements are affected by the periodic stiffeners, discussed in detail in Chapter 2.2 Ribs Section. Due to continuity at the fluid interface, the fluid term, $\mathrm{P}_{\mathrm{f}}$, must be modified in the same manner as the shell radial displacement. Recall that the radial displacement, $w$, is defined as:

$$
w=\sum_{m=-\infty}^{\infty} \sum_{n=0}^{\infty} W_{m n} \cos (n \theta) e^{i k_{m} z} e^{-i \omega t}
$$

To include the effects of ribs, the $\mathrm{P}_{\mathrm{f}}$ term is rewritten as:

$$
P_{f}=\sum_{m=-\infty}^{\infty} \sum_{n=0}^{\infty} R_{m n} H_{n}^{1}\left(\gamma_{m} r\right) \cos (n \theta) e^{i k_{m} z} e^{-i \omega t}
$$

$P_{i}$ does not change. $R_{m n}$ are unknown scattered wave coefficients; $k_{m}$ and $\gamma_{m}$ are both indexed to represent the periodicity of the reinforcing ribs.

$$
k_{m}=k_{z}+\frac{2 \pi m}{L}, \quad \gamma_{m}=\sqrt{k^{2}-k_{m}^{2}}
$$

The coefficients must first be dealt with before the fluid model can be used with the shell system. Conservation of linear momentum across the fluid interface requires that

$$
\rho_{f} \frac{\partial^{2} w}{\partial t^{2}}=-\left.\frac{\partial P_{a}}{\partial r}\right|_{r=c}
$$


Inputting the equations for displacement and fluid pressure into the momentum equation results in the following

$$
\begin{aligned}
-\rho_{f} \omega^{2} \sum_{m=-\infty}^{\infty} \sum_{n=0}^{\infty} W_{m n} \cos (n \theta) e^{i k_{m} z}=-P_{0} e^{i k_{z} z} \sum_{n=0}^{\infty} \varepsilon_{n} i^{n} \frac{\partial J_{n}\left(k_{r} r\right)}{\partial r} \cos (n \theta) \\
-\sum_{m=-\infty}^{\infty} \sum_{n=0}^{\infty} R_{m n} \frac{\partial H_{n}\left(\gamma_{m} r\right)}{\partial r} \cos (n \theta) e^{i k_{m} z}
\end{aligned}
$$

The $e^{-i \omega t}$ term is canceled. It is possible to isolate the entire $\sum_{m=-\infty}^{\infty} \sum_{n=0}^{\infty} R_{m n} \cos (n \theta) e^{i k_{m} z}$ term, via dividing by the Hankel derivative term. When dividing by the Hankel derivative, the summation in $m$ must be included. Derivatives of the Bessel and Hankel functions can be written as

$$
\begin{gathered}
\frac{\partial J_{n}\left(k_{r} r\right)}{\partial r}=\frac{k_{r}}{2}\left(J_{n-1}\left(k_{r} r\right)-J_{n+1}\left(k_{r} r\right)\right) \\
\frac{\partial H_{n}\left(\gamma_{m} r\right)}{\partial r}=\frac{\gamma_{m}}{2}\left(H_{n-1}\left(\gamma_{m} r\right)-H_{n+1}\left(\gamma_{m} r\right)\right)
\end{gathered}
$$

which are then substituted into Equation 4.3.9 and the unknown coefficients have been isolated.

$$
\begin{aligned}
\sum_{m=-\infty}^{\infty} \sum_{n=0}^{\infty} R_{m n} \cos (n \theta) e^{i k_{m} z} \\
=-P_{0} e^{i k_{z} z} \sum_{m=-\infty}^{\infty} \sum_{n=0}^{\infty} \varepsilon_{n} i^{n} \frac{k_{r}\left(J_{n-1}\left(k_{r} c\right)-J_{n+1}\left(k_{r} c\right)\right)}{\gamma_{m}\left(H_{n-1}\left(\gamma_{m} c\right)-H_{n+1}\left(\gamma_{m} c\right)\right)} \cos (n \theta) \\
\quad+\sum_{m=-\infty}^{\infty} \sum_{n=0}^{\infty}\left(\frac{2 \rho_{f} \omega^{2}}{\gamma_{m}}\right) \frac{W_{m n}}{\left(H_{n-1}\left(\gamma_{m} c\right)-H_{n+1}\left(\gamma_{m} c\right)\right)} \cos (n \theta) e^{i k_{m} z}
\end{aligned}
$$

Inserting the above into the pressure equation, $P_{a}$, gives the following

$$
\begin{gathered}
\mathrm{P}_{\mathrm{a}}=\mathrm{P}_{0} \mathrm{e}^{\mathrm{i} \mathrm{k}_{\mathrm{z}} \mathrm{z}} \sum_{\mathrm{n}=0}^{\infty} \varepsilon_{\mathrm{n}} \mathrm{i}^{\mathrm{n}} \mathrm{J}_{\mathrm{n}}\left(\mathrm{k}_{\mathrm{r}} \mathrm{c}\right) \cos (\mathrm{n} \theta) \\
-\mathrm{P}_{0} \mathrm{e}^{\mathrm{i} \mathrm{k}_{\mathrm{z}} \mathrm{z}} \sum_{\mathrm{m}=-\infty}^{\infty} \sum_{\mathrm{n}=0}^{\infty} \varepsilon_{\mathrm{n}} \mathrm{i}^{\mathrm{n}} \frac{\mathrm{k}_{\mathrm{r}}\left(\mathrm{J}_{\mathrm{n}-1}\left(\mathrm{k}_{\mathrm{r}} \mathrm{c}\right)-\mathrm{J}_{\mathrm{n}+1}\left(\mathrm{k}_{\mathrm{r}} \mathrm{c}\right)\right) \mathrm{H}_{\mathrm{n}}\left(\gamma_{\mathrm{m}} \mathrm{c}\right)}{\gamma_{\mathrm{m}}\left(\mathrm{H}_{\mathrm{n}-1}\left(\gamma_{\mathrm{m}} \mathrm{c}\right)-\mathrm{H}_{\mathrm{n}+1}\left(\gamma_{\mathrm{m}} \mathrm{c}\right)\right)} \cos (\mathrm{n} \theta) \\
+\sum_{\mathrm{m}=-\infty}^{\infty} \sum_{\mathrm{n}=0}^{\infty}\left(\frac{2 \rho_{\mathrm{f}} \omega^{2}}{\gamma_{\mathrm{m}}}\right) \frac{\mathrm{W}_{\mathrm{mn}} \mathrm{H}_{\mathrm{n}}\left(\gamma_{\mathrm{m}} \mathrm{c}\right)}{\left(\mathrm{H}_{\mathrm{n}-1}\left(\gamma_{\mathrm{m}} \mathrm{c}\right)-\mathrm{H}_{\mathrm{n}+1}\left(\gamma_{\mathrm{m}} \mathrm{c}\right)\right)} \cos (\mathrm{n} \theta) \mathrm{e}^{\mathrm{i} \mathrm{k}_{\mathrm{m}} \mathrm{z}}
\end{gathered}
$$

and the unknown coefficients $R_{m n}$ have been eliminated. Radial stress equations can now be reformulated taking into account the fluid pressure exerted on the exterior surface. 
As shown before, the pressure equation can be orthogonalized in both $\mathrm{n}$ and $\mathrm{m}$ to create an infinite set of $(n, m)$ decoupled equations, which are evaluated at $r=c$. Multiplying by $\mathrm{e}^{-\mathrm{i} \mathrm{k}_{\mathrm{m} 2} \mathrm{z}} \cos \left(\mathrm{n}_{2} \theta\right)$ and integrating from $[0,2 \pi]$ on $\mathrm{n}$ and $[0, L]$ on $\mathrm{z}$ results in the indexed set of equations

$$
\begin{aligned}
P_{a}[m, n]=P_{0} \varepsilon_{n} i^{n} & {\left[J_{n}\left(k_{r} c\right)-\frac{k_{r}\left(J_{n-1}\left(k_{r} c\right)-J_{n+1}\left(k_{r} c\right)\right) H_{n}\left(\gamma_{m} c\right)}{\gamma_{m}\left(H_{n-1}\left(\gamma_{m} c\right)-H_{n+1}\left(\gamma_{m} c\right)\right)}\right] \delta_{m 0} } \\
+ & \left(\frac{2 \rho_{f} \omega^{2}}{\gamma_{m}}\right) \frac{W_{m n} H_{n}\left(\gamma_{m} c\right)}{\left(H_{n-1}\left(\gamma_{m} c\right)-H_{n+1}\left(\gamma_{m} c\right)\right)}
\end{aligned}
$$

where $\delta_{m 0}$ is the Kroncker delta, which is 0 for all indices of $m$ expect $m=0$. The first term is referred to as the "blocked pressure" and is independent of the displacement coefficients, $W_{m n}$. The second term must be integrated into the dynamic A matrix of the system.

\subsubsection{Fluid Pressure Effect on Shell}

Due to the assumptions regarding viscosity the fluid cannot transmit shear forces with the shell, so only radial pressure is considered and only a single boundary condition $\left(\tau_{\mathrm{rr}}\right)$ is affected. 2.4.21 is rewritten as

$$
\begin{gathered}
\sum_{m=-\infty}^{\infty} \sum_{n=0}^{\infty}\left\{(\lambda+2 \mu) \frac{d W_{m n}(c)}{d r}+\frac{\lambda}{c} W_{m n}(c)+\frac{\lambda n}{c} V_{m n}(c)+\lambda i k_{m} U_{m n}(c)\right\} \cos (n \theta) e^{i k_{m} z} \\
+\sum_{m=-\infty}^{\infty} \sum_{n=0}^{\infty}\left(\frac{2 \rho_{f} \omega^{2}}{\gamma_{m}}\right) \frac{W_{m n} H_{n}\left(\gamma_{m} c\right)}{\left(H_{n-1}\left(\gamma_{m} c\right)-H_{n+1}\left(\gamma_{m} c\right)\right)} \cos (n \theta) e^{i k_{m} z} \\
=-P_{0} e^{i k_{z} z} \sum_{n=0}^{\infty} \varepsilon_{n} i^{n} J_{n}\left(k_{r} c\right) \cos (n \theta) \\
+P_{0} e^{i k_{z} z} \sum_{m=-\infty}^{\infty} \sum_{n=0}^{\infty} \varepsilon_{n} i^{n} \frac{k_{r}\left(J_{n-1}\left(k_{r} c\right)-J_{n+1}\left(k_{r} c\right)\right) H_{n}\left(\gamma_{m} c\right)}{\gamma_{m}\left(H_{n-1}\left(\gamma_{m} c\right)-H_{n+1}\left(\gamma_{m} c\right)\right)} \cos (n \theta)
\end{gathered}
$$

The second term of 4.3 .15 could be combined with the first term, but the equation will be orthogonalized in either case, and the decoupled result is given by 4.3 .14 . This leads to the $(\mathrm{m}$, n)-indexed 2.4.30 to be modified

$$
\begin{gathered}
(\lambda+2 \mu) \frac{d W_{m n}(c)}{d r}+\frac{\lambda}{c} W_{m n}(c)+\frac{\lambda n}{c} V_{m n}(c)+\lambda i k_{m} U_{m n}(c) \\
+\left(\frac{2 \rho_{f} \omega^{2}}{\gamma_{m}}\right) \frac{W_{m n}(c) H_{n}\left(\gamma_{m} c\right)}{\left(H_{n-1}\left(\gamma_{m} c\right)-H_{n+1}\left(\gamma_{m} c\right)\right)} \\
=-P_{0} \varepsilon_{n} i^{n}\left[J_{n}\left(k_{r} c\right)-\frac{k_{r}\left(J_{n-1}\left(k_{r} c\right)-J_{n+1}\left(k_{r} c\right)\right) H_{n}\left(\gamma_{m} c\right)}{\gamma_{m}\left(H_{n-1}\left(\gamma_{m} c\right)-H_{n+1}\left(\gamma_{m} c\right)\right)}\right] \delta_{m 0}
\end{gathered}
$$

To simplify 4.3.16, a fluid pressure gradient term is defined 


$$
\delta P_{f m}=\left(\frac{2 \rho_{f} \omega^{2}}{\gamma_{m}}\right) \frac{H_{n}\left(\gamma_{m} c\right)}{\left(H_{n-1}\left(\gamma_{m} c\right)-H_{n+1}\left(\gamma_{m} c\right)\right)}
$$

Inserting the unknown coefficients using 2.4.6 - 2.4.8 and 4.3.17 results in the final decoupled equation

$$
\begin{gathered}
{\left[\frac{2 \alpha \mu}{c} J_{n+1}\left(\alpha_{m} c\right)+\left(\frac{2 n(n-1) \mu}{c^{2}}-\alpha_{m}^{2} \lambda-2 \alpha_{m}^{2} \mu-k_{z m}^{2} \lambda\right) J_{n}\left(\alpha_{m} c\right)+\cdots\right.} \\
\left.+\left(-\alpha_{m} J_{n+1}\left(\alpha_{m} c\right)+\frac{n}{c} J_{n}\left(\alpha_{m} c\right)\right) \delta P_{f m}\right] A_{m n} \\
+\left[\frac{2 \alpha \mu}{c} Y_{n+1}\left(\alpha_{m} c\right)+\left(\frac{2 n(n-1) \mu}{c^{2}}-\alpha_{m}^{2} \lambda-2 \alpha_{m}^{2} \mu-k_{z m}^{2} \lambda\right) Y_{n}\left(\alpha_{m} c\right)+\cdots\right. \\
\left.+\left(-\alpha_{m} Y_{n+1}\left(\alpha_{m} c\right)+\frac{n}{c} Y_{n}\left(\alpha_{m} c\right)\right) \delta P_{f m}\right] B_{m n} \\
+\left[-\frac{2 n \beta_{m} \mu}{c} J_{n+1}\left(\beta_{m} c\right)+\frac{2 n(n-1) \mu}{c^{2}} J_{n}\left(\beta_{m} c\right)+\frac{n}{c} J_{n}\left(\beta_{m} c\right) \delta P_{f m}\right] C_{m n} \\
+\left[-\frac{2 n \beta_{m} \mu}{c} Y_{n+1}\left(\beta_{m} c\right)+\frac{2 n(n-1) \mu}{c^{2}} Y_{n}\left(\beta_{m} c\right)+\frac{n}{c} Y_{n}\left(\beta_{m} c\right) \delta P_{f m}\right] D_{m n} \\
+\left[-\frac{2 n(n+1) i k_{m} \mu}{c} J_{n+1}\left(\beta_{m} c\right)+2 i k_{m} \beta_{m} \mu J_{n}\left(\beta_{m} c\right)+i k_{m} J_{n+1}\left(\beta_{m} c\right) \delta P_{f m}\right] E_{m n} \\
+\left[-\frac{2 n(n+1) i k_{m} \mu}{c} Y_{n+1}\left(\beta_{m} c\right)+2 i k_{m} \beta_{m} \mu Y_{n}\left(\beta_{m} c\right)+i k_{m} Y_{n+1}\left(\beta_{m} c\right) \delta P_{f m}\right] F_{m n} \\
=-P_{0} \varepsilon_{n} i^{n}\left[J_{n}\left(k_{r} c\right)-\frac{k_{r}\left(J_{n-1}\left(k_{r} c\right)-J_{n+1}\left(k_{r} c\right)\right) H_{n}\left(\gamma_{m} c\right)}{\gamma_{m}\left(H_{n-1}\left(\gamma_{m} c\right)-H_{n+1}\left(\gamma_{m} c\right)\right)}\right] \delta \delta_{m 0}
\end{gathered}
$$

There are two important points to note. The first is that the new excitation form (last term of 4.3.18), which includes the original plane wave excitation as well as the interaction of the excitation with the fluid. This input is dependent on circumferential mode, $n$, which is why a less restrictive Kroncker delta is used (instead of $\delta_{m 0, n 0}$ ) compared to the ring load. Secondly, the fluid has a direct effect on the shell dynamics and will be included in $4^{\text {th }}$ row of the system A matrix. Finally, the excitation vector, $\mathbf{f}$, must also be modified to account for the blocked pressure

$$
\boldsymbol{f}=\left[0,0,0,-P_{0} \varepsilon_{n} i^{n}\left(J_{n}\left(k_{r} c\right)-\frac{k_{r}\left(J_{n-1}\left(k_{r} c\right)-J_{n+1}\left(k_{r} c\right)\right) H_{n}\left(\gamma_{m} c\right)}{\gamma_{m}\left(H_{n-1}\left(\gamma_{m} c\right)-H_{n+1}\left(\gamma_{m} c\right)\right)}\right), 0,0\right]^{T}
$$

All other parts of the global matrix are unchanged.

\subsubsection{Model Validation}

With the fluid model derived above implemented into the elastic shell model, the system can be validated against the reference, which also incorporates the same fluid model. Figures 4.3.1 and 4.3.2 give the spatial displacement fields for the ring loading $(k=0)$. 

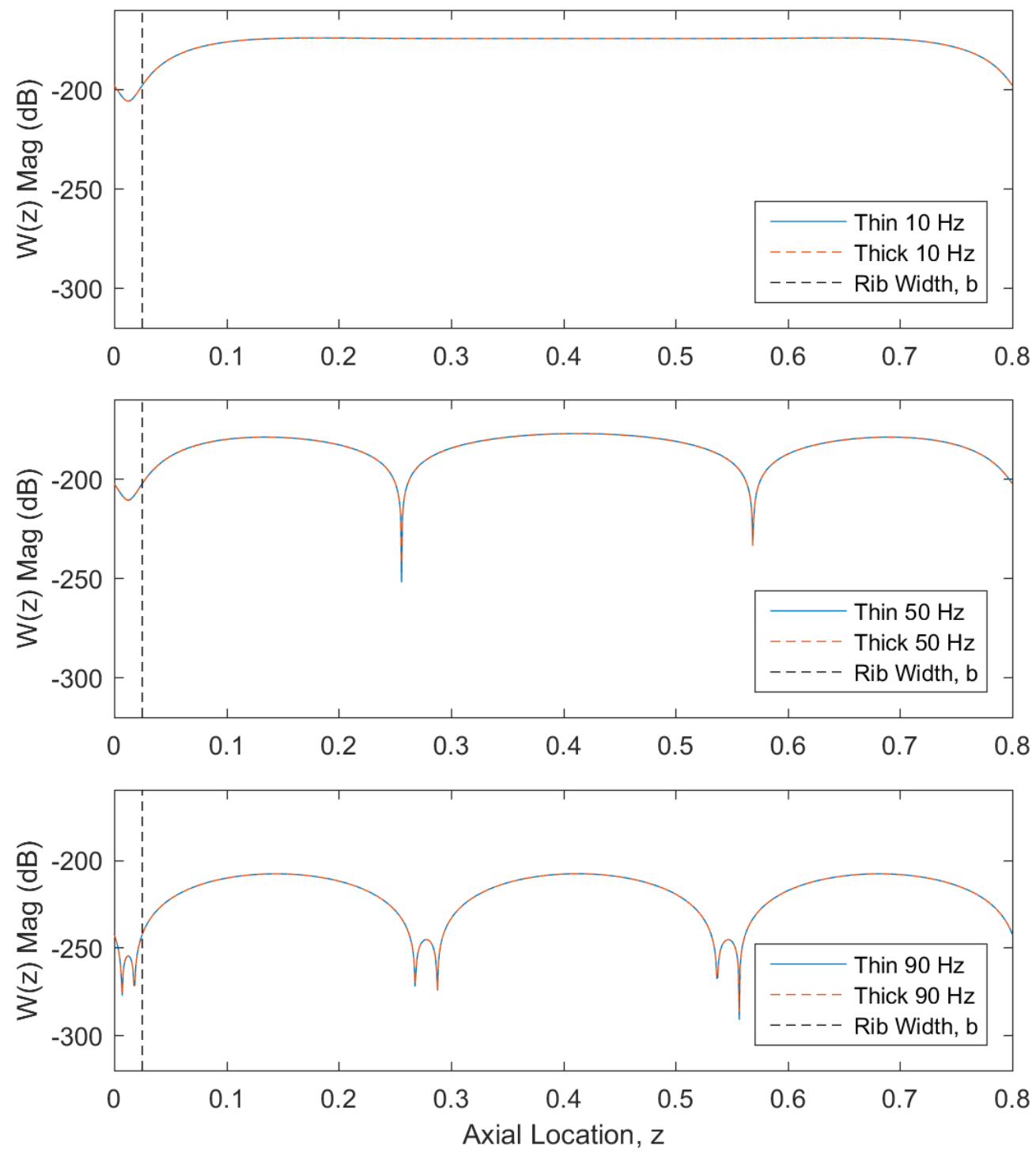

Figure 4.3.1 - Comparison of fluid reference and elastic radial displacement magnitude for ring load $(\mathrm{k}=0), \mathrm{m}_{\mathrm{pts}}=31$ 

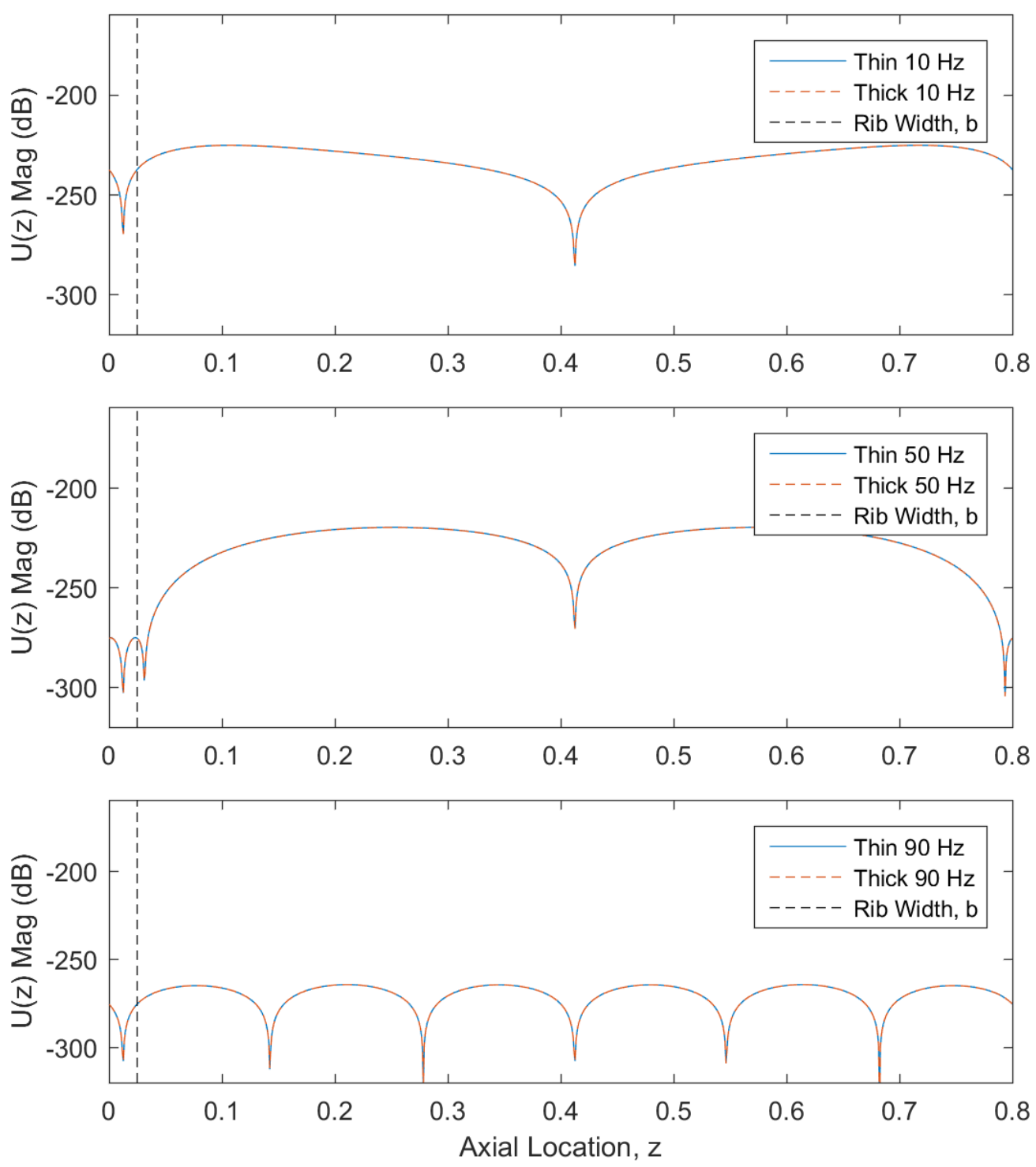

Figure 4.3.2 - Comparison of fluid reference and elastic axial displacement magnitude for ring load $(\mathrm{k}=0), \mathrm{m}_{\mathrm{pts}}=31$

Again, the elastic and reference model match very well, especially considering the small amplitudes. Even at frequencies below $100 \mathrm{~Hz}$, the fluid model has a significant effect on the spatial response of the periodic cylinder section. Displacements are much more sensitive to temporal excitation frequency. Scattered pressure is an additional measurement which the fluidloaded models can provide. Both the reflected pressure, denoted $P_{\text {inf }}$ because it assumed an infinite-impedance (rigid) system, and the radiated pressure, $P_{\text {rad, }}$ are output from the models. Scattered pressure can be measured at any radial distance from the system, but 1 meter from the outer surface is a typical measurement location and is chosen for this study. 

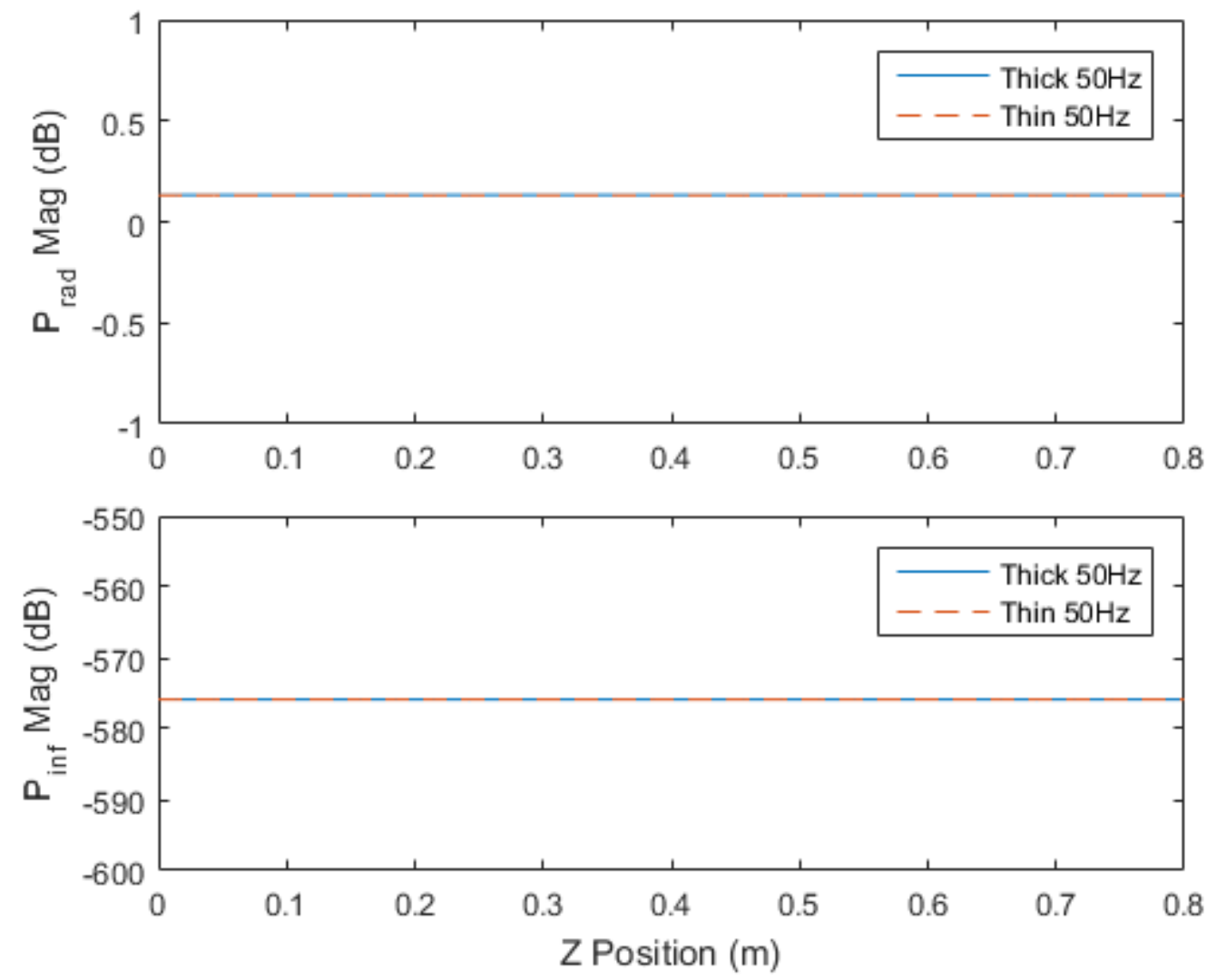

Figure 4.3.3 - Comparison of fluid reference and elastic radiated (top) and reflected (bottom) pressure magnitude for ring load $(\mathrm{k}=0), \mathrm{m}_{\mathrm{pts}}=31$

Radiated and reflected pressure both match very well, but have tremendously different scales. This is due to the nature of the ring load which propagates only in the axial direction. The ring load does cause significant radial shell deformation which radiates to the measurement location (1 meter from outer surface).

For the acoustic wave input, the normalized error is computer between the reference and the fluid-loaded elastic model. Figure 4.3.3 displays the errors in each displacement as an unwrapped cylinder; refer to Equation 3.1.8 for the expression for normalized error. 

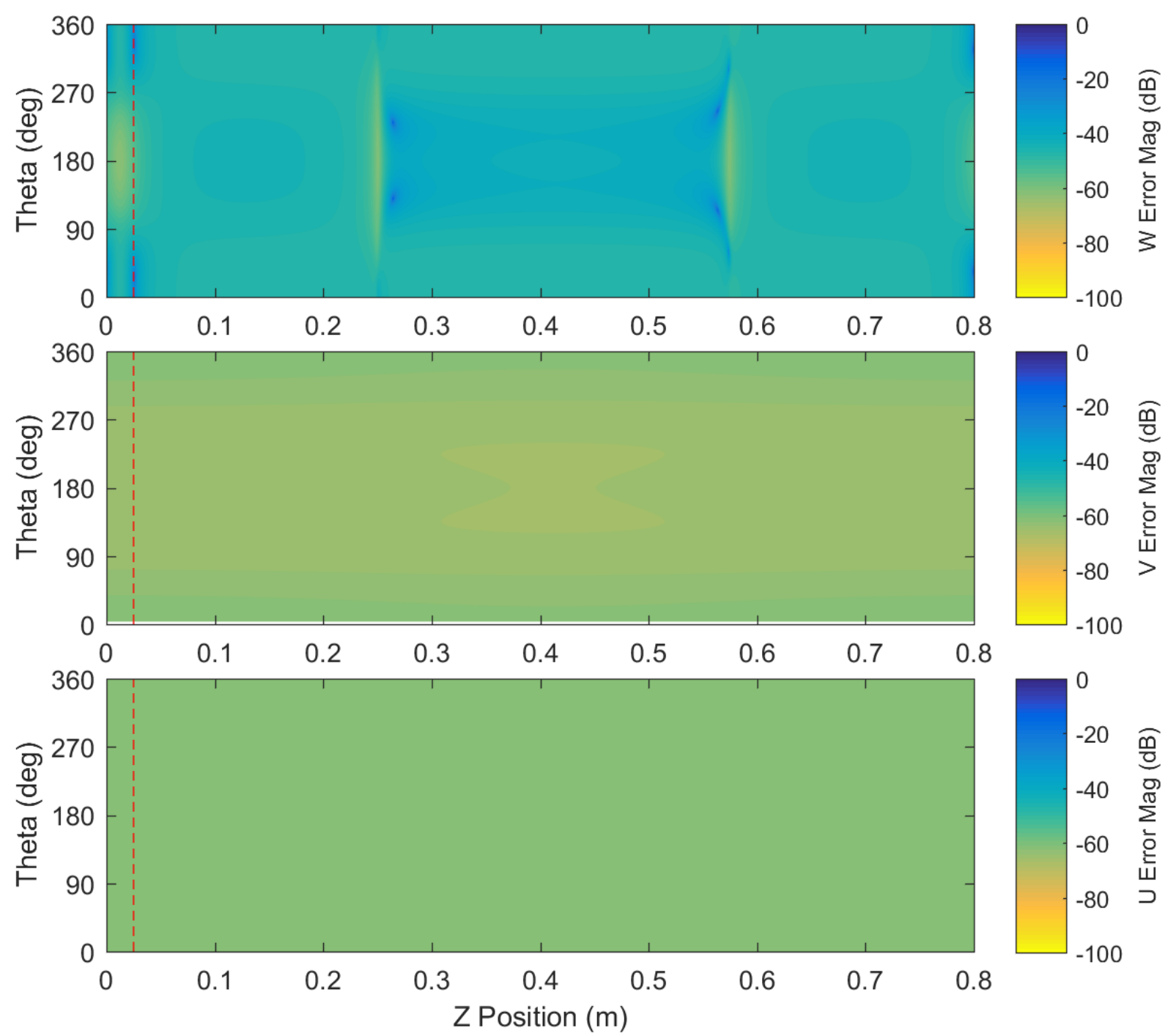

Figure 4.3.3 - Thin and Thick-shell radial (top), tangential (mid), and axial (bottom) Normalized Error in $\mathrm{dB}$ for plane-wave: $50 \mathrm{~Hz}, \varphi_{\mathrm{i}}=\pi / 12$, mpts $=31$, npts $=7$. Rib is located between the left edge $(z=0)$ and the red dashed line.

Tangential and axial errors are $-60 \mathrm{~dB}$ across the entire cylindrical section, representing approximately $0.1 \%$ error. Radial error again has 4 locations of high error $(>20 \%)$, which occur at displacement nodes; the nodes at $\theta=230 \mathrm{deg}$ is highlighted in Figure 4.3.4. 


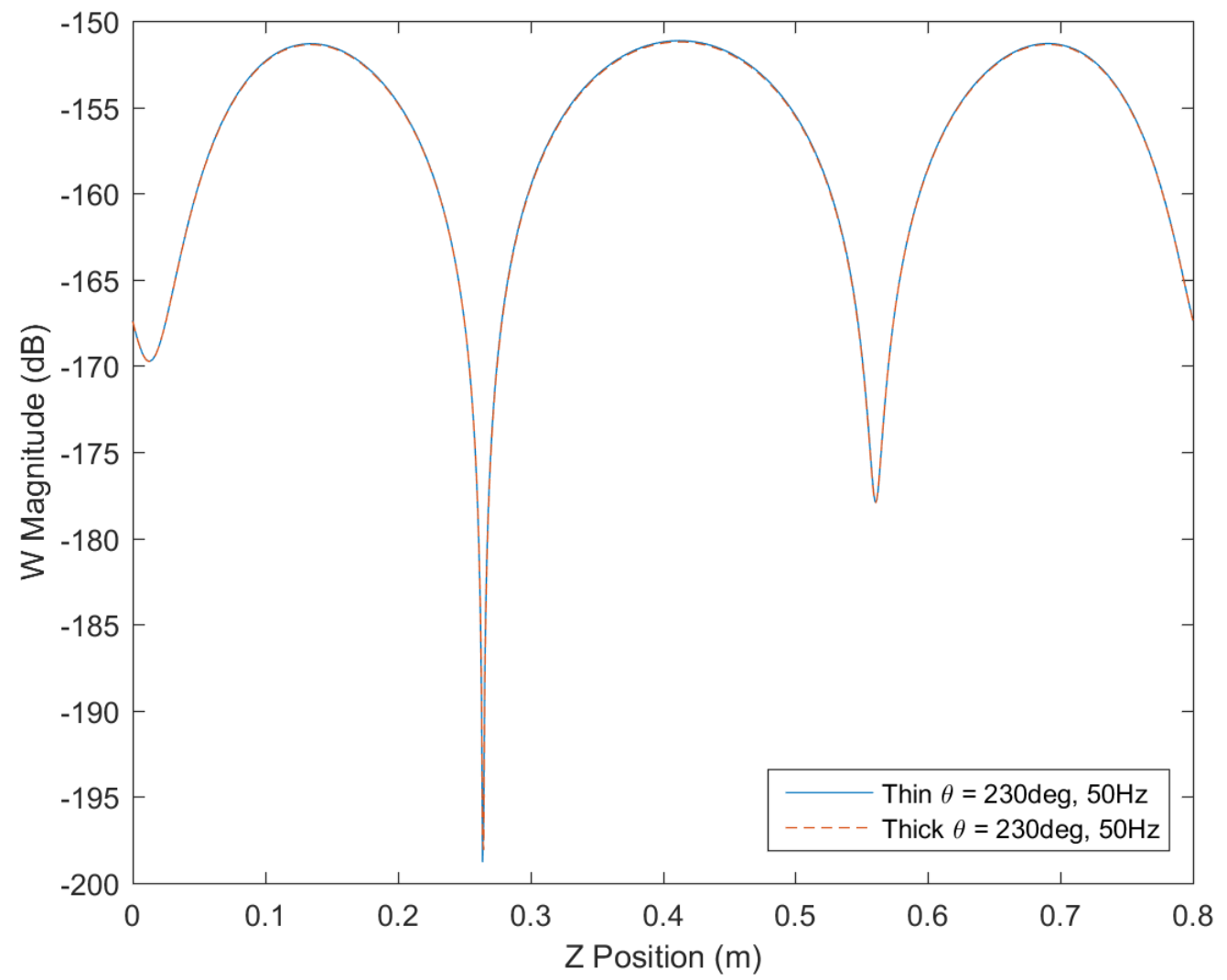

Figure 4.3.4 - Acoustic Wave radial response of reference and elastic model at $\theta=230 \mathrm{deg}$ Nodes are seen at positions $z=2.7$ and $5.6 \mathrm{~m}$, which lines up with the regions of high error in Figure 4.3.3. The normalized error is shown in Figure 4.3.5. 

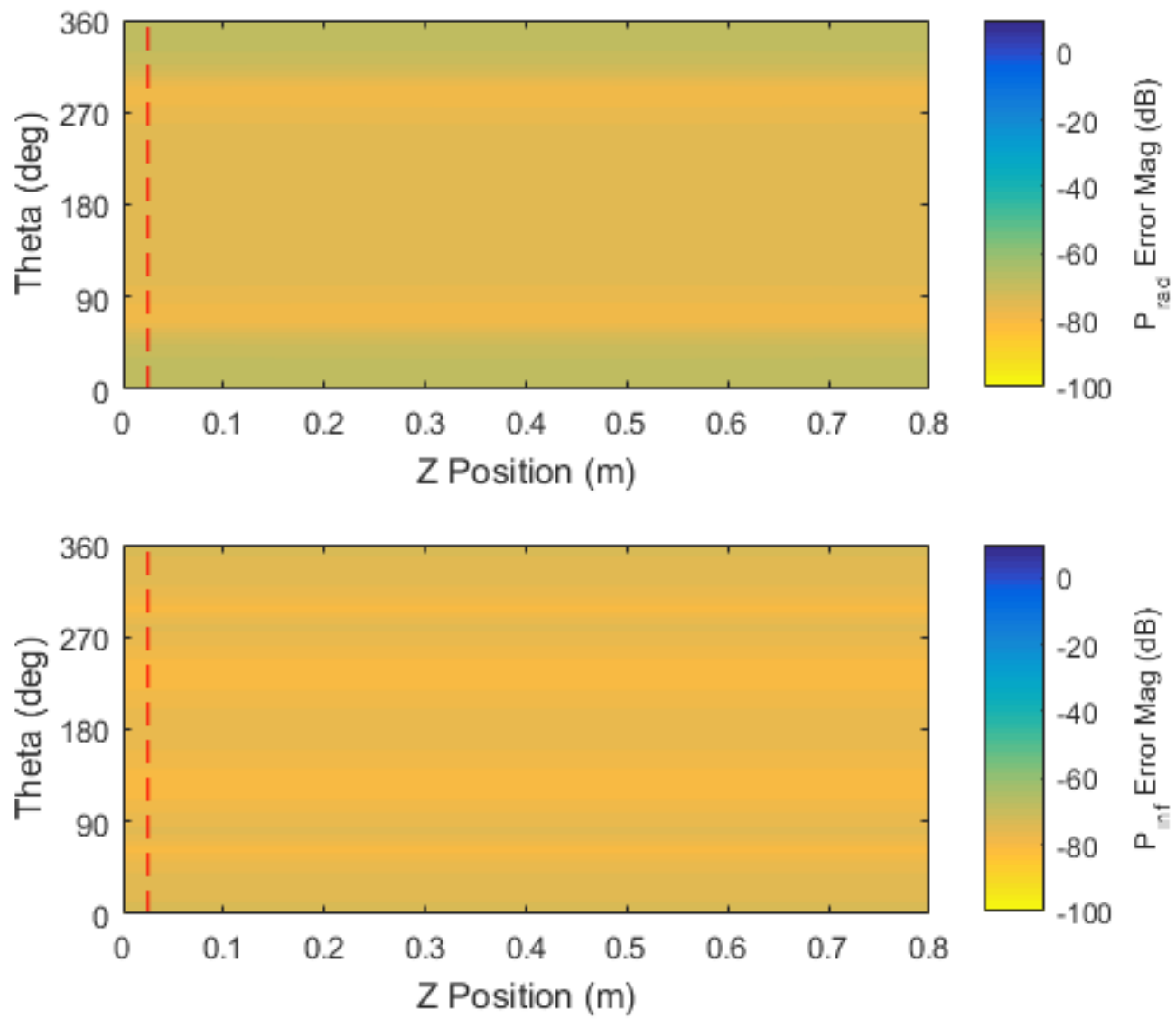

Figure 4.3.5 - Thin and Thick-shell radiated pressure (top) and reflected pressure (bottom) Normalized Error in $\mathrm{dB}$ for plane-wave: $50 \mathrm{~Hz}, \varphi_{\mathrm{i}}=\pi / 12$, mpts $=31$, npts $=7$. Rib is located between the left edge $(\mathrm{z}=0)$ and the red dashed line.

Error magnitude is less than $-70 \mathrm{~dB}$ for both pressures, indicating less than $0.05 \%$ error. Taking this into account, the elastic model with fluid-loading matches the reference well.

\subsection{Acoustic Coating}

\subsubsection{Acoustic Coating Derivation}

Combining the elastic shell model with an acoustic coating adds a significant layer of complexity to the base model described in Chapter 2.4. By design, the damping and stiffness properties of the coating significantly modify the overall system response to a disturbance, and acoustic coatings generally have a thickness large enough to warrant modeling as an independent elastic body. Thus the Navier-Cauchy elasticity equations are employed to describe both the shell as well as the coating behavior. Continuity equations for stress and displacement are used to 
model the shared interface between the bodies, and represents the adhesion of the coating on the shell. Displacement responses are found for the entire system, shell and coating.

The coated system derived here includes the elastic shell with finite-length Step connectors, with ribs are modeled as Timoshenko beams, and with fluid loading. Additional assumptions of the coating are: (1) the coating extends, along with the cylinder, infinitely along the cylindrical axis, (2) coating displacements are 3-dimensional and linear, (3) the coating material is homogenous and isotropic. Fluid pressure is applied to the outer coating surface $(r=$ $\left.c^{\prime}\right)$. Figure 4.4.1 displays an isometric view and Figure 4.4.2 displays a sidelong cross-section of the system.

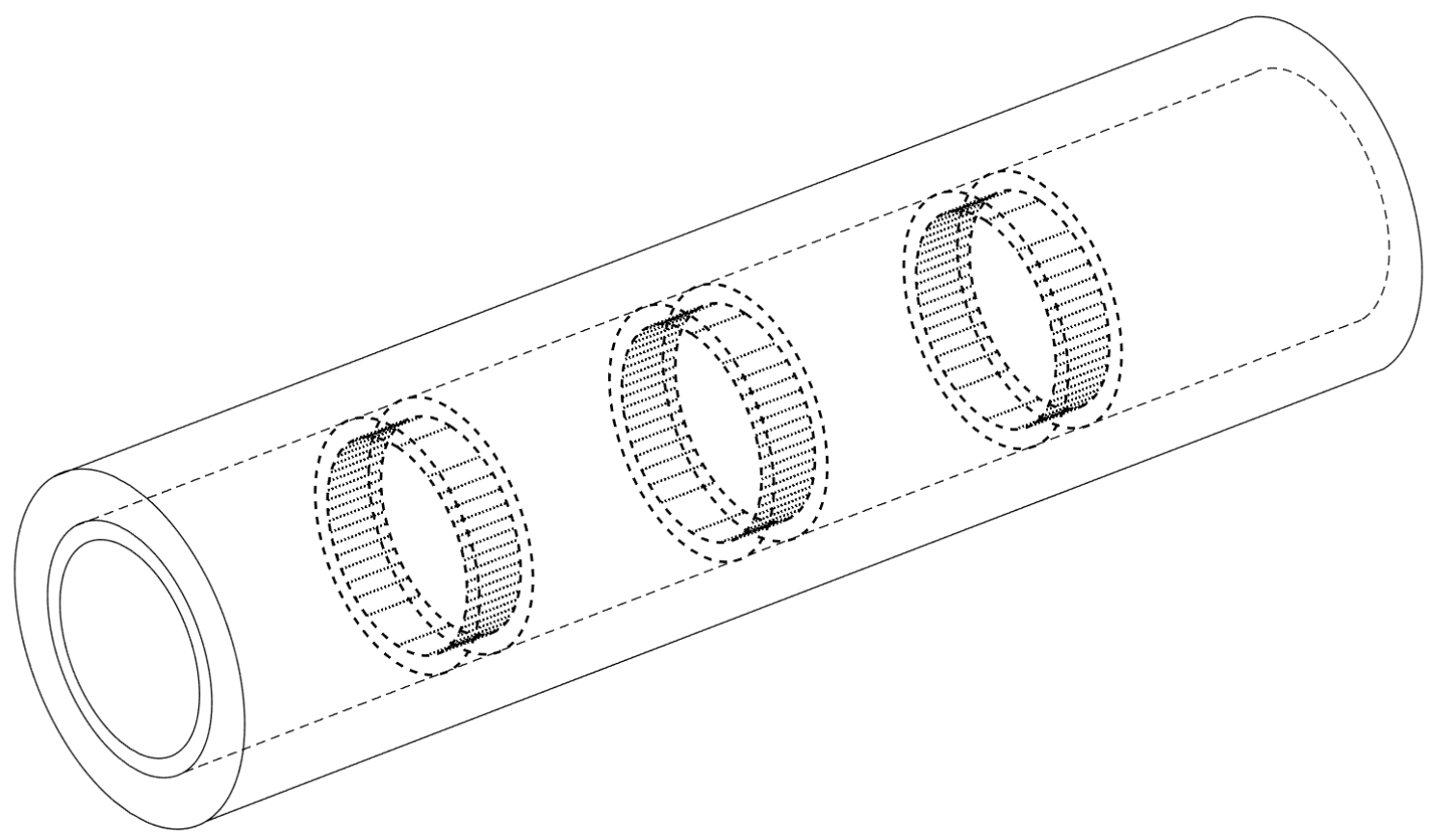

Figure 4.4.1 - Isometric view of the coated shell system

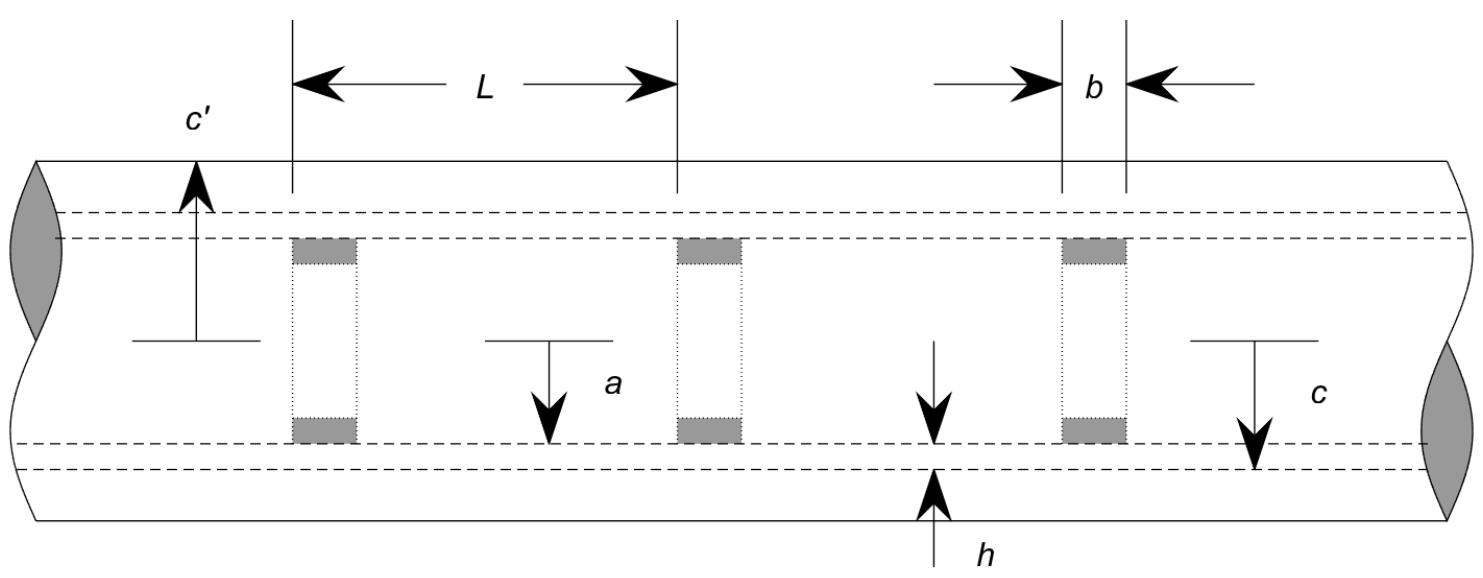

Figure 4.4.2 - Cross-section view of the coated shell system 
Boundary surfaces of the system are found at the inner shell surface $(r=a)$, the shell-coating interface $(r=c)$ and the outer coating surface $\left(r=c^{\prime}\right)$. Displacement of the shell (system 1) and coating (system 2 ) are denoted by $\mathbf{u}_{1}(r, \theta, z, t)$ and $\mathbf{u}_{2}(r, \theta, z, t)$, respectively. In cylindrical coordinates, the independent elasticity equations are

$$
\begin{aligned}
& \mu_{1} \nabla^{2} \mathbf{u}_{1}(r, \theta, z, t)+\left(\lambda_{1}+\mu_{1}\right) \nabla \nabla \cdot \mathbf{u}_{1}(r, \theta, z, t)=\rho_{1} \frac{\partial^{2} \mathbf{u}_{1}(r, \theta, z, t)}{\partial t^{2}} \\
& \mu_{2} \nabla^{2} \mathbf{u}_{2}(r, \theta, z, t)+\left(\lambda_{2}+\mu_{2}\right) \nabla \nabla \cdot \mathbf{u}_{2}(r, \theta, z, t)=\rho_{2} \frac{\partial^{2} \mathbf{u}_{2}(r, \theta, z, t)}{\partial t^{2}}
\end{aligned}
$$

Displacements, densities, and the Lamé parameters are specific to each system, which will be designated by the subscript $\mathrm{j}$. The reinforced shell is denoted by $\mathrm{j}=1$, the acoustic coating is denoted by $\mathrm{j}=2$. Displacement has the same form as Equations 2.4.2 -2.4 .4$

$$
\begin{aligned}
& w_{j}(r, \theta, z, t)=\sum_{m=-\infty}^{\infty} \sum_{n=0}^{\infty} W_{m n}^{(j)}(r) \cos (n \theta) e^{i k_{m} z} e^{-i \omega t} \\
& v_{j}(r, \theta, z, t)=\sum_{m=-\infty}^{\infty} \sum_{n=0}^{\infty} V_{m n}^{(j)}(r) \sin (n \theta) e^{i k_{m} z} e^{-i \omega t} \\
& u_{j}(r, \theta, z, t)=\sum_{m=-\infty}^{\infty} \sum_{n=0}^{\infty} U_{m n}^{(j)}(r) \cos (n \theta) e^{i k_{m} z} e^{-i \omega t}
\end{aligned}
$$

$W_{m n}, V_{m n}$, and $U_{m n}$ are the radial, tangential, and axial wave propagation coefficients specific to the shell $(j=1)$ or coating $(j=2)$, and each are comprised of unknown coefficients

$$
\begin{gathered}
W_{m n}^{(j)}(r)=A_{m n}^{(j)}\left[-\alpha_{m}^{(j)} J_{n+1}\left(\alpha_{m}^{(j)} r\right)+\frac{n}{r} J_{n}\left(\alpha_{m}^{(j)} r\right)\right]+B_{m n}^{(j)}\left[-\alpha_{m}^{(j)} Y_{n+1}\left(\alpha_{m}^{(j)} r\right)+\frac{n}{r} Y_{n}\left(\alpha_{m}^{(j)} r\right)\right] \\
+C_{m n}^{(j)} \frac{n}{r} J_{n}\left(\beta_{m}^{(j)} r\right)+D_{m n}^{(j)} \frac{n}{r} Y_{n}\left(\beta_{m}^{(j)} r\right)+E_{m n}^{(j)} i k_{m} J_{n+1}\left(\beta_{m}^{(j)} r\right)+F_{m n}^{(j)} i k_{m} Y_{n+1}\left(\beta_{m}^{(j)} r\right) \\
V_{m n}^{(j)}(r)=-A_{m n}^{(j)} \frac{n}{r} J_{n}\left(\alpha_{m}^{(j)} r\right)-B_{m n}^{(j)} \frac{n}{r} Y_{n}\left(\alpha_{m}^{(j)} r\right)+C_{m n}^{(j)}\left[\beta_{m}^{(j)} J_{n+1}\left(\beta_{m}^{(j)} r\right)-\frac{n}{r} J_{n}\left(\beta_{m}^{(j)} r\right)\right] \\
+D_{m n}^{(j)}\left[\beta_{m}^{(j)} Y_{n+1}\left(\beta_{m}^{(j)} r\right)-\frac{n}{r} Y_{n}\left(\beta_{m}^{(j)} r\right)\right]+E_{m n}^{(j)} i k_{m} J_{n+1}\left(\beta_{m}^{(j)} r\right)+F_{m n}^{(j)} i k_{m} Y_{n+1}\left(\beta_{m}^{(j)} r\right) \\
U_{m n}^{(j)}(r)=A_{m n}^{(j)} i k_{m} J_{n}\left(\alpha_{m}^{(j)} r\right)+B_{m n}^{(j)} i k_{m} Y_{n}\left(\alpha_{m}^{(j)} r\right) \\
-E_{m n}^{(j)} \beta_{m}^{(j)} J_{n}\left(\beta_{m}^{(j)} r\right)-F_{m n}^{(j)} \beta_{m}^{(j)} Y_{n}\left(\beta_{m}^{(j)} r\right)
\end{gathered}
$$

Note that the modified dilatational and shear wavenumbers are also distinct to each body

$$
\alpha_{m}^{(j)}=\sqrt{\left(\frac{\omega}{c_{1}}\right)^{2}-k_{m}^{2}}, \quad \beta_{m}^{(j)}=\sqrt{\left(\frac{\omega}{c_{2}}\right)^{2}-k_{m}^{2}}
$$


where the wave speeds can be expanded in terms of Lamé parameters

$$
c_{1}^{2}=\frac{\lambda_{j}+2 \mu_{j}}{\rho_{j}}, \quad c_{2}^{2}=\frac{\mu_{j}}{\rho_{j}}
$$

\subsubsection{Evaluating the Boundary Conditions}

There are three boundary surfaces in the model noted in Figures 4.4.1 and 4.4.2: the reinforced inner shell surface $(r=a)$, the shell-coating interface $(r=c)$, and the outer fluid-loaded surface $\left(r=c^{\prime}\right)$. The inner shell surface can be treated similarly to the thick-shell model of Chapter 2.4, and the outer surface boundary condition was derived in Chapter 4.3. However, at the shared interface, continuity of stress and displacement require a new set of equations. Continuity of stress $\tau^{(1)}=\tau^{(2)}$ is given as

$$
\begin{aligned}
\tau_{r r}^{(1)}(c, \theta, z, t) & =\tau_{r r}^{(2)}(c, \theta, z, t) \\
\tau_{r \theta}^{(1)}(c, \theta, z, t) & =\tau_{r \theta}^{(2)}(c, \theta, z, t) \\
\tau_{r z}^{(1)}(c, \theta, z, t) & =\tau_{r z}^{(2)}(c, \theta, z, t)
\end{aligned}
$$

and continuity of displacement at the interface requires that

$$
\begin{aligned}
& w_{1}(c, \theta, z, t)=w_{2}(c, \theta, z, t) \\
& v_{1}(c, \theta, z, t)=v_{2}(c, \theta, z, t) \\
& u_{1}(c, \theta, z, t)=u_{2}(c, \theta, z, t)
\end{aligned}
$$

At the inner surface $(r=a)$, the shell stresses are equal to the external stiffener stresses resulting in 3 equations, originally derived as Equations 2.4.12 - 2.4.14. Similarly, at the outer coating surface $\left(r=c^{\prime}\right)$, radial stress is equal to fluid pressure and applied excitation, while the tangential and axial stresses are zero. Combining Equations 4.4.11-4.4.16 with the 3 inner equations and 3 outer equations leads to the following 12 equation system

$$
\begin{gathered}
\left(\lambda_{1}+2 \mu_{1}\right) \frac{\partial w_{1}}{\partial r}+\frac{\lambda_{1}}{a} w_{1}+\frac{\lambda_{1}}{a} \frac{\partial v_{1}}{\partial \theta}+\lambda_{1} \frac{\partial u_{1}}{\partial z}=f_{r} \\
\mu_{1} \frac{\partial v_{1}}{\partial r}-\frac{\mu_{1}}{a} v_{1}+\frac{\mu_{1}}{a} \frac{\partial w_{1}}{\partial \theta}=f_{\theta} \\
\mu_{1} \frac{\partial w_{1}}{\partial z}+\mu_{1} \frac{\partial u_{1}}{\partial r}=f_{z} \\
\left(\lambda_{1}+2 \mu_{1}\right) \frac{\partial w_{1}}{\partial r}+\frac{\lambda_{1}}{c} w_{1}+\frac{\lambda_{1}}{c} \frac{\partial v_{1}}{\partial \theta}+\lambda_{1} \frac{\partial u_{1}}{\partial z} \\
=\left(\lambda_{2}+2 \mu_{2}\right) \frac{\partial w_{2}}{\partial r}+\frac{\lambda_{2}}{c} w_{2}+\frac{\lambda_{2}}{c} \frac{\partial v_{2}}{\partial \theta}+\lambda_{2} \frac{\partial u_{2}}{\partial z}
\end{gathered}
$$




$$
\begin{aligned}
& \mu_{1} \frac{\partial v_{1}}{\partial r}-\frac{\mu_{1}}{c} v_{1}+\frac{\mu_{1}}{c} \frac{\partial w_{1}}{\partial \theta}=\mu_{2} \frac{\partial v_{2}}{\partial r}-\frac{\mu_{2}}{c} v_{2}+\frac{\mu_{1}}{c} \frac{\partial w_{1}}{\partial \theta} \\
& \mu_{1} \frac{\partial w_{1}}{\partial z}+\mu_{1} \frac{\partial u_{1}}{\partial r}=\mu_{2} \frac{\partial w_{2}}{\partial z}+\mu_{2} \frac{\partial u_{2}}{\partial r} \\
& w_{1}(c, \theta, z, t)=w_{2}(c, \theta, z, t) \\
& v_{1}(c, \theta, z, t)=v_{2}(c, \theta, z, t) \\
& u_{1}(c, \theta, z, t)=u_{2}(c, \theta, z, t) \\
&\left(\lambda_{2}+2 \mu_{2}\right) \frac{\partial w_{2}}{\partial r}+\frac{\lambda_{2}}{c^{\prime}} w_{2}+\frac{\lambda_{2}}{c^{\prime}} \frac{\partial v_{2}}{\partial \theta}+\lambda_{2} \frac{\partial u_{2}}{\partial z}=-P_{a} \\
& \mu_{2} \frac{\partial v_{2}}{\partial r}-\frac{\mu_{2}}{c^{\prime}} v_{2}+\frac{\mu_{2}}{c^{\prime}} \frac{\partial w_{2}}{\partial \theta}=0 \\
& \mu_{2} \frac{\partial w_{2}}{\partial z}+\mu_{2} \frac{\partial u_{2}}{\partial r}=0
\end{aligned}
$$

Building off of the models derived in Chapter 2.4 (thick-shell), 4.1 (step connectors), 4.2 (beam ribs), and 4.3 (fluid loading), the system of equations $4.4 .17-4.4 .28$ is written as

$$
\begin{aligned}
& \sum_{m=-\infty}^{\infty} \sum_{n=0}^{\infty}\left\{\left(\lambda_{1}+2 \mu_{1}\right) \frac{d W_{m n}^{(1)}(a)}{d r}+\frac{\lambda_{1}}{a} W_{m n}^{(1)}(a)+\frac{\lambda_{1} n}{a} V_{m n}^{(1)}(a)+\lambda_{1} i k_{m} U_{m n}^{(1)}(a)\right\} \cos (n \theta) e^{-i k_{m} z} \\
& =\frac{1}{a b} \sum_{m=-\infty}^{\infty} \sum_{n=0}^{\infty}\left\{K_{r w} W_{m n}^{(1)}+K_{r v} V_{m n}^{(1)}\right\} \cos (n \theta) e^{-i k_{m} z} \sum_{s=-\infty}^{\infty}[H(z-s L)-H(z-b-s L)] \\
& =\frac{1}{a b} \sum_{m=-\infty} \sum_{n=0}^{\infty}\left\{K_{\theta w} W_{m n}^{(1)}+K_{\theta v} V_{m n}^{(1)}\right\} \sin (n \theta) e^{-i k_{m} z} \sum_{s=-\infty}^{\infty}[H(z-s L)-H(z-b-s L)] \\
& =\frac{1}{a b} \sum_{m=-\infty}^{\infty} \sum_{n=0}^{\infty}\left\{K_{z w} W_{m n}^{(1)}\right\} \cos (q \theta) e^{i k_{m} z} \sum_{s=-\infty}^{\infty}[H(z-s L)-H(z-b-S L)]
\end{aligned}
$$




$$
\begin{aligned}
\sum_{m=-\infty}^{\infty} \sum_{n=0}^{\infty}\left\{\left(\lambda_{1}\right.\right. & \left.\left.+2 \mu_{1}\right) \frac{d W_{m n}^{(1)}(c)}{d r}+\frac{\lambda_{1}}{c} W_{m n}^{(1)}(c)+\frac{\lambda_{1} n}{c} V_{m n}^{(1)}(c)+\lambda_{1} i k_{m} U_{m n}^{(1)}(c)\right\} \cos (n \theta) e^{-i k_{m} z} \\
& =\sum_{m=-\infty}^{\infty} \sum_{n=0}^{\infty}\left\{\left(\lambda_{2}+2 \mu_{2}\right) \frac{d W_{m n}^{(2)}(c)}{d r}+\frac{\lambda_{2}}{c} W_{m n}^{(2)}(c)+\frac{\lambda_{2} n}{c} V_{m n}^{(2)}(c)\right. \\
& \left.+\lambda_{2} i k_{m} U_{m n}^{(2)}(c)\right\} \cos (n \theta) e^{-i k_{m} z}
\end{aligned}
$$$$
\begin{aligned}
& \sum_{m=-\infty}^{\infty} \sum_{n=0}^{\infty}\left\{\mu_{1} \frac{d V_{m n}^{(1)}(c)}{d r}-\frac{\mu_{1}}{c} V_{m n}^{(1)}(c)-\frac{\mu_{1} n}{c} W_{m n}^{(1)}(c)\right\} \sin (n \theta) e^{-i k_{m} z} \\
= & \sum_{m=-\infty}^{\infty} \sum_{n=0}^{\infty}\left\{\mu_{2} \frac{d V_{m n}^{(2)}(c)}{d r}-\frac{\mu_{2}}{c} V_{m n}^{(2)}(c)-\frac{\mu_{2} n}{c} W_{m n}^{(2)}(c)\right\} \sin (n \theta) e^{-i k_{m} z}
\end{aligned}
$$$$
\sum_{m=-\infty}^{\infty} \sum_{n=0}^{\infty}\left\{\mu_{1} i k_{m} W_{m n}^{(1)}(c)+\mu_{1} \frac{d U_{m n}^{(1)}(c)}{d r}\right\} \cos (n \theta) e^{-i k_{m} z}
$$$$
=\sum_{m=-\infty}^{\infty} \sum_{n=0}^{\infty}\left\{\mu_{2} i k_{m} W_{m n}^{(2)}(c)+\mu_{2} \frac{d U_{m n}^{(2)}(c)}{d r}\right\} \cos (n \theta) e^{-i k_{m} z}
$$$$
\sum_{m=-\infty}^{\infty} \sum_{n=0}^{\infty}\left\{W_{m n}^{(1)}(c)\right\} \cos (n \theta) e^{-i k_{m} z}=\sum_{m=-\infty}^{\infty} \sum_{n=0}^{\infty}\left\{W_{m n}^{(2)}(c)\right\} \cos (n \theta) e^{-i k_{m} z}
$$$$
\sum_{m=-\infty}^{\infty} \sum_{n=0}^{\infty}\left\{V_{m n}^{(1)}(c)\right\} \sin (n \theta) e^{-i k_{m} z}=\sum_{m=-\infty}^{\infty} \sum_{n=0}^{\infty}\left\{V_{m n}^{(2)}(c)\right\} \sin (n \theta) e^{-i k_{m} z}
$$$$
\sum_{m=-\infty}^{\infty} \sum_{n=0}^{\infty}\left\{U_{m n}^{(1)}(c)\right\} \cos (n \theta) e^{-i k_{m} z}=\sum_{m=-\infty}^{\infty} \sum_{n=0}^{\infty}\left\{U_{m n}^{(2)}(c)\right\} \cos (n \theta) e^{-i k_{m} z}
$$$$
\sum_{m=-\infty}^{\infty} \sum_{n=0}^{\infty}\left\{\left(\lambda_{2}+2 \mu_{2}\right) \frac{d W_{m n}^{(2)}\left(c^{\prime}\right)}{d r}+\frac{\lambda_{2}}{c^{\prime}} W_{m n}^{(2)}\left(c^{\prime}\right)+\frac{\lambda_{2} n}{c^{\prime}} V_{m n}^{(2)}\left(c^{\prime}\right)\right.
$$$$
\left.+\lambda_{2} i k_{m} U_{m n}^{(2)}\left(c^{\prime}\right)+\delta P_{f m} W_{m n}^{(2)}\left(c^{\prime}\right)\right\} \cos (n \theta) e^{i k_{m} z}
$$$$
=-P_{0} e^{i k_{z} z} \sum_{n=0}^{\infty} \varepsilon_{n} i^{n} J_{n}\left(k_{r} c^{\prime}\right) \cos (n \theta)
$$$$
+P_{0} e^{i k_{z} z} \sum_{m=-\infty}^{\infty} \sum_{n=0}^{\infty} \varepsilon_{n} i^{n} \frac{k_{r}\left(J_{n-1}\left(k_{r} c^{\prime}\right)-J_{n+1}\left(k_{r} c^{\prime}\right)\right) H_{n}\left(\gamma_{m} c^{\prime}\right)}{\gamma_{m}\left(H_{n-1}\left(\gamma_{m} c^{\prime}\right)-H_{n+1}\left(\gamma_{m} c^{\prime}\right)\right)} \cos (n \theta)
$$ 


$$
\begin{gathered}
\sum_{m=-\infty}^{\infty} \sum_{n=0}^{\infty}\left\{\mu_{2} \frac{d V_{m n}^{(2)}\left(c^{\prime}\right)}{d r}-\frac{\mu_{2}}{c^{\prime}} V_{m n}^{(2)}\left(c^{\prime}\right)-\frac{\mu_{2} n}{c^{\prime}} W_{m n}^{(2)}\left(c^{\prime}\right)\right\} \sin (n \theta) e^{i k_{m} z}=0 \\
\sum_{m=-\infty}^{\infty} \sum_{n=0}^{\infty}\left\{\mu_{2} i k_{m} W_{m n}^{(2)}\left(c^{\prime}\right)+\mu_{2} \frac{d U_{m n}^{(2)}\left(c^{\prime}\right)}{d r}\right\} \cos (n \theta) e^{i k_{m} z}=0
\end{gathered}
$$

Before decoupling the 12 system equations into their indexed sets, it is necessary to make the same changes to the summation of stiffener terms: taking the Fourier series of the step functions using 2.2.17-2.2.18 and then shifting the index of the results using 2.3.16.

\subsubsection{Mode Indexed and Global System}

Transforming the system of 12 equations with infinite summations into mode decoupled sets involves the same orthogonalization process used throughout this study. Next, the 6 displacement coefficients ( $\mathrm{W}, \mathrm{V}, \mathrm{U}$ for each body) are replaced with the 12 unknown coefficients according to $4.4 .6-4.4 .8$, which leads to the $(m, n)$-indexed set

$$
\begin{aligned}
& {\left[\frac{2 \alpha_{m}^{(1)} \mu_{1}}{a} J_{n+1}\left(\alpha_{m}^{(1)} a\right)+\left(\frac{2 n(n-1) \mu_{1}}{a^{2}}-\alpha_{m}^{2(1)} \lambda_{1}-2 \alpha_{m}^{2(1)} \mu_{1}-k_{z m}^{2} \lambda_{1}\right) J_{n}\left(\alpha_{m}^{(1)} a\right)\right] A_{m n}^{(1)}} \\
& +\left[\frac{2 \alpha_{m}^{(1)} \mu}{a} Y_{n+1}\left(\alpha_{m}^{(1)} a\right)+\left(\frac{2 n(n-1) \mu_{1}}{a^{2}}-\alpha_{m}^{2(1)} \lambda_{1}-2 \alpha_{m}^{2(1)} \mu_{1}-k_{z m}^{2} \lambda_{1}\right) Y_{n}\left(\alpha_{m} a\right)\right] B_{m n}^{(1)} \\
& +\left[-\frac{2 n \beta_{m} \mu_{1}}{a} J_{n+1}\left(\beta_{m} a\right)+\frac{2 n(n-1) \mu_{1}}{a^{2}} J_{n}\left(\beta_{m} a\right)\right] C_{m n}^{(1)} \\
& +\left[-\frac{2 n \beta_{m} \mu_{1}}{a} Y_{n+1}\left(\beta_{m} a\right)+\frac{2 n(n-1) \mu_{1}}{a^{2}} Y_{n}\left(\beta_{m} a\right)\right] D_{m n}^{(1)} \\
& +\left[-\frac{2 n(n+1) i k_{m} \mu_{1}}{a} J_{n+1}\left(\beta_{m} a\right)+2 i k_{m} \beta_{m} \mu_{1} J_{n}\left(\beta_{m} a\right)\right] E_{m n}^{(1)} \\
& a \\
& +\frac{1}{a b} \sum_{s=-\infty}^{\infty} K_{r w}\left\{\left[-\alpha_{s} J_{n+1}\left(\alpha_{s} a\right)+\frac{n}{r} J_{n}\left(\alpha_{s} a\right)\right] A_{s n}^{(1)}+\left[-\alpha_{s} Y_{n+1}\left(\alpha_{s} a\right)+\frac{n}{a} Y_{n}\left(\alpha_{s} a\right)\right] B_{s n}^{(1)}\right. \\
& \left.+\frac{n}{a} J_{n}\left(\beta_{s} a\right) C_{s n}+\frac{n}{a} Y_{n}\left(\beta_{s} a\right) D_{s n}^{(1)}+i k_{s} J_{n+1}\left(\beta_{s} a\right) E_{s n}^{(1)}+i k_{s} Y_{n+1}\left(\beta_{s} a\right) F_{s n}^{(1)}\right\} d_{m-s} \\
& +K_{r v}\left\{-\frac{n}{a} J_{n}\left(\alpha_{s} a\right) A_{s n}^{(1)}-\frac{n}{a} Y_{n}\left(\alpha_{s} a\right) B_{s n}^{(1)}+\left[\beta_{s} J_{n+1}\left(\beta_{s} a\right)-\frac{n}{a} J_{n}\left(\beta_{s} a\right)\right] C_{s n}^{(1)}\right. \\
& \left.+\left[\beta_{s} Y_{n+1}\left(\beta_{s} a\right)-\frac{n}{a} Y_{n}\left(\beta_{s} a\right)\right] D_{s n}^{(1)}+i k_{s} J_{n+1}\left(\beta_{s} a\right) E_{s n}^{(1)}+i k_{s} Y_{n+1}\left(\beta_{s} a\right) F_{s n}^{(1)}\right\} d_{m-s}
\end{aligned}
$$




$$
\begin{aligned}
& {\left[\frac{2 n \alpha_{m} \mu_{1}}{a} J_{n+1}\left(\alpha_{m} a\right)-\frac{2 n(n-1) \mu_{1}}{a^{2}} J_{n}\left(\alpha_{m} a\right)\right] A_{m n}^{(1)}} \\
& +\left[\frac{2 n \alpha_{m} \mu_{1}}{a} Y_{n+1}\left(\alpha_{m} a\right)-\frac{2 n(n-1) \mu_{1}}{a^{2}} Y_{n}\left(\alpha_{m} a\right)\right] B_{m n}^{(1)} \\
& +\left[-\frac{\beta_{m} \mu_{1}}{a} J_{n+1}\left(\beta_{m} a\right)+\left(\beta_{m}^{2} \mu_{1}-\frac{2 n(n-1) \mu_{1}}{a^{2}}\right) J_{n}\left(\beta_{m} a\right)\right] C_{m n}^{(1)} \\
& +\left[-\frac{\beta_{m} \mu_{1}}{a} Y_{n+1}\left(\beta_{m} a\right)+\left(\beta_{m}^{2} \mu_{1}-\frac{2 n(n-1) \mu_{1}}{a^{2}}\right) Y_{n}\left(\beta_{m} a\right)\right] D_{m n}^{(1)} \\
& +\left[-\frac{2(n+1) i k_{m} \mu_{1}}{a} J_{n+1}\left(\beta_{m} a\right)+i k_{m} \beta_{m} \mu_{1} J_{n}\left(\beta_{m} a\right)\right] E_{m n}^{(1)} \\
& +\left[-\frac{2(n+1) i k_{m} \mu_{1}}{a} Y_{n+1}\left(\beta_{m} a\right)+i k_{m} \beta_{m} \mu_{1} Y_{n}\left(\beta_{m} a\right)\right] F_{m n}^{(1)} \\
& =\frac{1}{a b} \sum_{s=-\infty}^{\infty} K_{\theta v}\left\{-\frac{n}{a} J_{n}\left(\alpha_{s} a\right) A_{s n}^{(1)}-\frac{n}{a} Y_{n}\left(\alpha_{s} a\right) B_{s n}^{(1)}+\left[\beta_{s} J_{n+1}\left(\beta_{s} a\right)-\frac{n}{a} J_{n}\left(\beta_{s} a\right)\right] C_{s n}^{(1)}\right. \\
& \left.+\left[\beta_{s} Y_{n+1}\left(\beta_{s} a\right)-\frac{n}{a} Y_{n}\left(\beta_{s} a\right)\right] D_{s n}^{(1)}+i k_{s} J_{n+1}\left(\beta_{s} a\right) E_{s n}^{(1)}+i k_{s} Y_{n+1}\left(\beta_{s} a\right) F_{s n}^{(1)}\right\} d_{m-s} \\
& K_{r w}\left\{\left[-\alpha_{s} J_{n+1}\left(\alpha_{s} a\right)+\frac{n}{r} J_{n}\left(\alpha_{s} a\right)\right] A_{s n}^{(1)}+\left[-\alpha_{s} Y_{n+1}\left(\alpha_{s} a\right)+\frac{n}{a} Y_{n}\left(\alpha_{s} a\right)\right] B_{s n}^{(1)}\right. \\
& \left.+\frac{n}{a} J_{n}\left(\beta_{s} a\right) C_{s n}^{(1)}+\frac{n}{a} Y_{n}\left(\beta_{s} a\right) D_{s n}^{(1)}+i k_{s} J_{n+1}\left(\beta_{s} a\right) E_{s n}^{(1)}+i k_{s} Y_{n+1}\left(\beta_{s} a\right) F_{s n}^{(1)}\right\} d_{m-s} \\
& {\left[-2 i k_{m} \alpha_{m} J_{n+1}\left(\alpha_{m} a\right)+\frac{2 n i k_{m} \mu_{1}}{a} J_{n}\left(\alpha_{m} a\right)\right] A_{m n}^{(1)}} \\
& +\left[-2 i k_{m} \alpha_{m} Y_{n+1}\left(\alpha_{m} a\right)+\frac{2 n i k_{m} \mu_{1}}{a} Y_{n}\left(\alpha_{m} a\right)\right] B_{m n}^{(1)} \\
& +\left[\frac{n i k_{m} \mu_{1}}{a} J_{n}\left(\beta_{m} a\right)\right] C_{m n}+\left[\frac{n i k_{m} \mu_{1}}{a} Y_{n}\left(\beta_{m} a\right)\right] D_{m n}^{(1)} \\
& +\left[\mu_{1}\left(\beta_{m}^{2}-k_{m}^{2}\right) J_{n+1}\left(\beta_{m} a\right)-\frac{n \beta_{m} \mu_{1}}{a} J_{n}\left(\beta_{m} a\right)\right] E_{m n}^{(1)} \\
& +\left[\mu_{1}\left(\beta_{m}^{2}-k_{m}^{2}\right) Y_{n+1}\left(\beta_{m} a\right)-\frac{n \beta_{m} \mu_{1}}{a} Y_{n}\left(\beta_{m} a\right)\right] F_{m n}^{(1)} \\
& =\frac{1}{a b} \sum_{s=-\infty}^{\infty} K_{r w}\left\{\left[-\alpha_{s} J_{n+1}\left(\alpha_{s} a\right)+\frac{n}{r} J_{n}\left(\alpha_{s} a\right)\right] A_{s n}^{(1)}+\left[-\alpha_{s} Y_{n+1}\left(\alpha_{s} a\right)+\frac{n}{a} Y_{n}\left(\alpha_{s} a\right)\right] B_{s n}^{(1)}\right. \\
& \left.+\frac{n}{a} J_{n}\left(\beta_{s} a\right) C_{s n}^{(1)}+\frac{n}{a} Y_{n}\left(\beta_{s} a\right) D_{s n}^{(1)}+i k_{s} J_{n+1}\left(\beta_{s} a\right) E_{s n}^{(1)}+i k_{s} Y_{n+1}\left(\beta_{s} a\right) F_{s n}^{(1)}\right\} d_{m-s}
\end{aligned}
$$




$$
\begin{gathered}
{\left[\frac{2 \alpha_{m}^{(1)} \mu_{1}}{c} J_{n+1}\left(\alpha_{m}^{(1)} c\right)+\left(\frac{2 n(n-1) \mu_{1}}{c^{2}}-\alpha_{m}^{2(1)} \lambda_{1}-2 \alpha_{m}^{2(1)} \mu_{1}-k_{z m}^{2} \lambda_{1}\right) J_{n}\left(\alpha_{m}^{(1)} c\right)\right] A_{m n}^{(1)}} \\
+\left[\frac{2 \alpha_{m}^{(1)} \mu_{1}}{c} Y_{n+1}\left(\alpha_{m}^{(1)} c\right)+\left(\frac{2 n(n-1) \mu_{1}}{c^{2}}-\alpha_{m}^{2(1)} \lambda_{1}-2 \alpha_{m}^{2(1)} \mu_{1}-k_{z m}^{2} \lambda_{1}\right) Y_{n}\left(\alpha_{m} c\right)\right] B_{m n}^{(1)} \\
+\left[-\frac{2 n \beta_{m} \mu_{1}}{c} J_{n+1}\left(\beta_{m} c\right)+\frac{2 n(n-1) \mu_{1}}{c^{2}} J_{n}\left(\beta_{m} c\right)\right] C_{m n}^{(1)} \\
+\left[-\frac{2 n \beta_{m} \mu_{1}}{c} Y_{n+1}\left(\beta_{m} c\right)+\frac{2 n(n-1) \mu_{1}}{c^{2}} Y_{n}\left(\beta_{m} c\right)\right] D_{m n}^{(1)} \\
+\left[-\frac{2 n(n+1) i k_{m} \mu_{1}}{c} J_{n+1}\left(\beta_{m} c\right)+2 i k_{m} \beta_{m} \mu_{1} J_{n}\left(\beta_{m} c\right)\right] E_{m n}^{(1)} \\
+\left[-\frac{2 n(n+1) i k_{m} \mu_{1}}{c} Y_{n+1}\left(\beta_{m} c\right)+2 i k_{m} \beta_{m} \mu_{1} Y_{n}\left(\beta_{m} c\right)\right] F_{m n}^{(1)} \\
=\left[\frac{2 \alpha_{m}^{(2)} \mu_{2}}{c} J_{n+1}\left(\alpha_{m}^{(2)} c\right)+\left(\frac{2 n(n-1) \mu_{2}}{c^{2}}-\alpha_{m}^{(2) 2} \lambda_{2}-2 \alpha_{m}^{2(2)} \mu_{2}-k_{z m}^{2} \lambda_{2}\right) J_{n}\left(\alpha_{m}^{(2)} c\right)\right] A_{m n}^{(2)} \\
+\left[\frac{2 \alpha_{m}^{(2)} \mu_{2}}{c} Y_{n+1}\left(\alpha_{m}^{(2)} c\right)+\left(\frac{2 n(n-1) \mu_{2}}{c^{2}}-\alpha_{m}^{(2) 2} \lambda_{2}-2 \alpha_{m}^{2(2)} \mu_{2}-k_{z m}^{2} \lambda_{2}\right) Y_{n}\left(\alpha_{m}^{(2)} c\right)\right] B_{m n}^{(2)} \\
+\left[-\frac{2 n \beta_{m} \mu_{2}}{c} J_{n+1}\left(\beta_{m} c\right)+\frac{2 n(n-1) \mu_{2}}{c^{2}} J_{n}\left(\beta_{m} c\right)\right] C_{m n}^{(2)} \\
+\left[-\frac{2 n \beta_{m} \mu_{2}}{c} Y_{n+1}\left(\beta_{m} c\right)+\frac{2 n(n-1) \mu_{2}}{c^{2}} Y_{n}\left(\beta_{m} c\right)\right] D_{m n}^{(2)} \\
+\left[-\frac{2 n(n+1) i k_{m} \mu_{2}}{c} J_{n+1}\left(\beta_{m} c\right)+2 i k_{m} \beta_{m} \mu_{2} J_{n}\left(\beta_{m} c\right)\right] E_{m n}^{(2)} \\
+\left[-\frac{2 n(n+1) i k_{m} \mu_{2}}{c} Y_{n+1}\left(\beta_{m} c\right)+2 i k_{m} \beta_{m} \mu_{2} Y_{n}\left(\beta_{m} c\right)\right] F_{m n}^{(2)} \\
+\left[\begin{array}{l}
2 n(4) .5) \\
+
\end{array}\right.
\end{gathered}
$$




$$
\begin{aligned}
& {\left[\frac{2 n \alpha_{m} \mu_{1}}{c} J_{n+1}\left(\alpha_{m} c\right)-\frac{2 n(n-1) \mu_{1}}{c^{2}} J_{n}\left(\alpha_{m} c\right)\right] A_{m n}^{(1)} } \\
& +\left[\frac{2 n \alpha_{m} \mu_{1}}{c} Y_{n+1}\left(\alpha_{m} c\right)-\frac{2 n(n-1) \mu_{1}}{c^{2}} Y_{n}\left(\alpha_{m} c\right)\right] B_{m n}^{(1)} \\
+ & {\left[-\frac{\beta_{m} \mu_{1}}{c} J_{n+1}\left(\beta_{m} c\right)+\left(\beta_{m}^{2} \mu_{1}-\frac{2 n(n-1) \mu_{1}}{c^{2}}\right) J_{n}\left(\beta_{m} c\right)\right] C_{m n}^{(1)} } \\
+ & {\left[-\frac{\beta_{m} \mu_{1}}{c} Y_{n+1}\left(\beta_{m} c\right)+\left(\beta_{m}^{2} \mu_{1}-\frac{2 n(n-1) \mu_{1}}{c^{2}}\right) Y_{n}\left(\beta_{m} c\right)\right] D_{m n}^{(1)} } \\
+ & {\left[-\frac{2(n+1) i k_{m} \mu_{1}}{c} J_{n+1}\left(\beta_{m} c\right)+i k_{m} \beta_{m} \mu_{1} J_{n}\left(\beta_{m} c\right)\right] E_{m n}^{(1)} } \\
+ & {\left[-\frac{2(n+1) i k_{m} \mu_{1}}{c} Y_{n+1}\left(\beta_{m} c\right)+i k_{m} \beta_{m} \mu_{1} Y_{n}\left(\beta_{m} c\right)\right] F_{m n}^{(1)} } \\
& =\left[\frac{2 n \alpha_{m} \mu_{2}}{c} J_{n+1}\left(\alpha_{m} c\right)-\frac{2 n(n-1) \mu_{2}}{c^{2}} J_{n}\left(\alpha_{m} c\right)\right] A_{m n}^{(2)} \\
+ & +\left[\frac{2 n \alpha_{m} \mu_{2}}{c} Y_{n+1}\left(\alpha_{m} c\right)-\frac{2 n(n-1) \mu_{2}}{c^{2}} Y_{n}\left(\alpha_{m} c\right)\right] B_{m n}^{(2)} \\
+ & {\left[-\frac{\beta_{m} \mu_{2}}{c} J_{n+1}\left(\beta_{m} c\right)+\left(\beta_{m}^{2} \mu_{2}-\frac{2 n(n-1) \mu_{2}}{c^{2}}\right) J_{n}\left(\beta_{m} c\right)\right] C_{m n}^{(2)} } \\
+ & {\left[-\frac{\beta_{m} \mu_{2}}{c} Y_{n+1}\left(\beta_{m} c\right)+\left(\beta_{m}^{2} \mu_{2}-\frac{2 n(n-1) \mu_{2}}{c^{2}}\right) Y_{n}\left(\beta_{m} c\right)\right] D_{m n}^{(2)} } \\
+ & {\left[-\frac{2(n+1) i k_{m} \mu_{2}}{c} J_{n+1}\left(\beta_{m} c\right)+i k_{m} \beta_{m} \mu_{2} J_{n}\left(\beta_{m} c\right)\right] E_{m n}^{(2)} }
\end{aligned}
$$




$$
\begin{aligned}
& \quad\left[-2 i k_{m} \alpha_{m} J_{n+1}\left(\alpha_{m} c\right)+\frac{2 n i k_{m} \mu_{1}}{c} J_{n}\left(\alpha_{m} c\right)\right] A_{m n}^{(1)} \\
& +\left[-2 i k_{m} \alpha_{m} Y_{n+1}\left(\alpha_{m} c\right)+\frac{2 n i k_{m} \mu_{1}}{c} Y_{n}\left(\alpha_{m} c\right)\right] B_{m n}^{(1)} \\
& +\left[\frac{n i k_{m} \mu_{1}}{c} J_{n}\left(\beta_{m} c\right)\right] C_{m n}+\left[\frac{n i k_{m} \mu_{1}}{c} Y_{n}\left(\beta_{m} c\right)\right] D_{m n}^{(1)} \\
& +\left[\mu_{1}\left(\beta_{m}^{2}-k_{m}^{2}\right) J_{n+1}\left(\beta_{m} c\right)-\frac{n \beta_{m} \mu_{1}}{c} J_{n}\left(\beta_{m} c\right)\right] E_{m n}^{(1)} \\
& +\left[\mu_{1}\left(\beta_{m}^{2}-k_{m}^{2}\right) Y_{n+1}\left(\beta_{m} c\right)-\frac{n \beta_{m} \mu_{1}}{c} Y_{n}\left(\beta_{m} c\right)\right] F_{m n}^{(1)} \\
& =\left[-2 i k_{m} \alpha_{m} J_{n+1}\left(\alpha_{m} c\right)+\frac{2 n i k_{m} \mu_{2}}{c} J_{n}\left(\alpha_{m} c\right)\right] A_{m n}^{(2)} \\
& +\left[-2 i k_{m} \alpha_{m} Y_{n+1}\left(\alpha_{m} c\right)+\frac{2 n i k_{m} \mu_{2}}{c} Y_{n}\left(\alpha_{m} c\right)\right] B_{m n}^{(2)} \\
& +\left[\frac{n i k_{m} \mu_{2}}{c} J_{n}\left(\beta_{m} c\right)\right] C_{m n}+\left[\frac{n i k_{m} \mu_{2}}{c} Y_{n}\left(\beta_{m} c\right)\right] D_{m n}^{(2)} \\
& +\left[\mu_{2}\left(\beta_{m}^{2}-k_{m}^{2}\right) J_{n+1}\left(\beta_{m} c\right)-\frac{n \beta_{m} \mu_{2}}{c} J_{n}\left(\beta_{m} c\right)\right] E_{m n}^{(2)} \\
& +\left[\mu_{2}\left(\beta_{m}^{2}-k_{m}^{2}\right) Y_{n+1}\left(\beta_{m} c\right)-\frac{n \beta_{m} \mu_{2}}{c} Y_{n}\left(\beta_{m} c\right)\right] F_{m n}^{(2)}
\end{aligned}
$$

$$
\begin{aligned}
& {\left[-\alpha_{m}^{(1)} J_{n+1}\left(\alpha_{m}^{(1)} c\right)+\frac{n}{c} J_{n}\left(\alpha_{m}^{(1)} c\right)\right] A_{m n}^{(1)}+\left[-\alpha_{m}^{(1)} Y_{n+1}\left(\alpha_{m}^{(1)} c\right)+\frac{n}{c} Y_{n}\left(\alpha_{m}^{(1)} c\right)\right] B_{m n}^{(1)} } \\
+ & \frac{n}{c} J_{n}\left(\beta_{m}^{(1)} c\right) C_{m n}^{(1)}+\frac{n}{c} Y_{n}\left(\beta_{m}^{(1)} c\right) D_{m n}^{(1)}+i k_{m} J_{n+1}\left(\beta_{m}^{(1)} c\right) E_{m n}^{(1)}+i k_{m} Y_{n+1}\left(\beta_{m}^{(1)} c\right) F_{m n}^{(1)} \\
= & {\left[-\alpha_{m}^{(2)} J_{n+1}\left(\alpha_{m}^{(2)} c\right)+\frac{n}{c} J_{n}\left(\alpha_{m}^{(2)} c\right)\right] A_{m n}^{(2)}+\left[-\alpha_{m}^{(2)} Y_{n+1}\left(\alpha_{m}^{(2)} c\right)+\frac{n}{c} Y_{n}\left(\alpha_{m}^{(2)} c\right)\right] B_{m n}^{(2)} } \\
+ & \frac{n}{c} J_{n}\left(\beta_{m}^{(2)} c\right) C_{m n}^{(2)}+\frac{n}{c} Y_{n}\left(\beta_{m}^{(2)} c\right) D_{m n}^{(2)}+i k_{m} J_{n+1}\left(\beta_{m}^{(2)} c\right) E_{m n}^{(2)}+i k_{m} Y_{n+1}\left(\beta_{m}^{(2)} c\right) F_{m n}^{(2)} \\
& -\frac{n}{c} J_{n}\left(\alpha_{m}^{(1)} c\right) A_{m n}^{(1)}-\frac{n}{c} Y_{n}\left(\alpha_{m}^{(1)} c\right) B_{m n}^{(1)}+\left[\beta_{m}^{(1)} J_{n+1}\left(\beta_{m}^{(1)} c\right)-\frac{n}{c} J_{n}\left(\beta_{m}^{(1)} c\right)\right] C_{m n}^{(1)} \\
+ & {\left[\beta_{m}^{(1)} Y_{n+1}\left(\beta_{m}^{(1)} c\right)-\frac{n}{c} Y_{n}\left(\beta_{m}^{(1)} c\right)\right] D_{m n}^{(1)}+i k_{m} J_{n+1}\left(\beta_{m}^{(1)} c\right) E_{m n}^{(1)}+i k_{m} Y_{n+1}\left(\beta_{m}^{(1)} c\right) F_{m n}^{(1)} } \\
& =-\frac{n}{c} J_{n}\left(\alpha_{m}^{(1)} c\right) A_{m n}^{(1)}-\frac{n}{c} Y_{n}\left(\alpha_{m}^{(1)} c\right) B_{m n}^{(1)}+\left[\beta_{m}^{(1)} J_{n+1}\left(\beta_{m}^{(1)} c\right)-\frac{n}{c} J_{n}\left(\beta_{m}^{(1)} c\right)\right] C_{m n}^{(1)} \\
+ & {\left[\beta_{m}^{(1)} Y_{n+1}\left(\beta_{m}^{(1)} c\right)-\frac{n}{c} Y_{n}\left(\beta_{m}^{(1)} c\right)\right] D_{m n}^{(1)}+i k_{m} J_{n+1}\left(\beta_{m}^{(1)} c\right) E_{m n}^{(1)}+i k_{m} Y_{n+1}\left(\beta_{m}^{(1)} c\right) F_{m n}^{(1)} }
\end{aligned}
$$




$$
\begin{aligned}
& i k_{m} J_{n}\left(\alpha_{m}^{(1)} c\right) A_{m n}^{(1)}+i k_{m} Y_{n}\left(\alpha_{m}^{(1)} c\right) B_{m n}^{(1)}-\beta_{m}^{(1)} J_{n}\left(\beta_{m}^{(1)} c\right) E_{m n}^{(1)}-\beta_{m}^{(1)} Y_{n}\left(\beta_{m}^{(1)} c\right) F_{m n}^{(1)} \\
& =i k_{m} J_{n}\left(\alpha_{m}^{(2)} c\right) A_{m n}^{(2)}+i k_{m} Y_{n}\left(\alpha_{m}^{(2)} c\right) B_{m n}^{(2)}-\beta_{m}^{(2)} J_{n}\left(\beta_{m}^{(2)} c\right) E_{m n}^{(2)}-\beta_{m}^{(2)} Y_{n}\left(\beta_{m}^{(2)} c\right) F_{m n}^{(2)} \\
& {\left[\frac{2 \alpha \mu_{2}}{c^{\prime}} J_{n+1}\left(\alpha_{m} c^{\prime}\right)+\left(\frac{2 n(n-1) \mu_{2}}{c^{\prime 2}}-\alpha_{m}^{2} \lambda_{2}-2 \alpha_{m}^{2} \mu_{2}-k_{z m}^{2} \lambda_{2}\right) J_{n}\left(\alpha_{m} c^{\prime}\right)+\cdots\right.} \\
& \left.+\left(-\alpha_{m} J_{n+1}\left(\alpha_{m} c^{\prime}\right)+\frac{n}{c^{\prime}} J_{n}\left(\alpha_{m} c^{\prime}\right)\right) \delta P_{f m}\right] A_{m n}^{(2)} \\
& +\left[\frac{2 \alpha \mu_{2}}{c^{\prime}} Y_{n+1}\left(\alpha_{m} c^{\prime}\right)+\left(\frac{2 n(n-1) \mu_{2}}{c^{\prime 2}}-\alpha_{m}^{2} \lambda_{2}-2 \alpha_{m}^{2} \mu_{2}-k_{z m}^{2} \lambda_{2}\right) Y_{n}\left(\alpha_{m} c^{\prime}\right)+\cdots\right. \\
& \left.+\left(-\alpha_{m} Y_{n+1}\left(\alpha_{m} c^{\prime}\right)+\frac{n}{c^{\prime}} Y_{n}\left(\alpha_{m} c^{\prime}\right)\right) \delta P_{f m}\right] B_{m n}^{(2)} \\
& +\left[-\frac{2 n \beta_{m} \mu_{2}}{c^{\prime}} J_{n+1}\left(\beta_{m} c^{\prime}\right)+\frac{2 n(n-1) \mu_{2}}{c^{\prime 2}} J_{n}\left(\beta_{m} c^{\prime}\right)+\frac{n}{c^{\prime}} J_{n}\left(\beta_{m} c^{\prime}\right) \delta P_{f m}\right] C_{m n}^{(2)} \\
& +\left[-\frac{2 n \beta_{m} \mu_{2}}{c^{\prime}} Y_{n+1}\left(\beta_{m} c^{\prime}\right)+\frac{2 n(n-1) \mu_{2}}{c^{\prime 2}} Y_{n}\left(\beta_{m} c^{\prime}\right)+\frac{n}{c^{\prime}} Y_{n}\left(\beta_{m} c^{\prime}\right) \delta P_{f m}\right] D_{m n}^{(2)} \\
& +\left[-\frac{2 n(n+1) i k_{m} \mu_{2}}{c^{\prime}} J_{n+1}\left(\beta_{m} c^{\prime}\right)+2 i k_{m} \beta_{m} \mu_{2} J_{n}\left(\beta_{m} c^{\prime}\right)+i k_{m} J_{n+1}\left(\beta_{m} c^{\prime}\right) \delta P_{f m}\right] E_{m n}^{(2)} \\
& +\left[-\frac{2 n(n+1) i k_{m} \mu_{2}}{c^{\prime}} Y_{n+1}\left(\beta_{m} c^{\prime}\right)+2 i k_{m} \beta_{m} \mu_{2} Y_{n}\left(\beta_{m} c^{\prime}\right)+i k_{m} Y_{n+1}\left(\beta_{m} c^{\prime}\right) \delta P_{f m}\right] F_{m n}^{(2)} \\
& =-P_{0} \varepsilon_{n} i^{n}\left[J_{n}\left(k_{r} c^{\prime}\right)-\frac{k_{r}\left(J_{n-1}\left(k_{r} c^{\prime}\right)-J_{n+1}\left(k_{r} c^{\prime}\right)\right) H_{n}\left(\gamma_{m} c^{\prime}\right)}{\gamma_{m}\left(H_{n-1}\left(\gamma_{m} c^{\prime}\right)-H_{n+1}\left(\gamma_{m} c^{\prime}\right)\right)}\right] \delta_{m 0} \\
& {\left[\frac{2 n \alpha \mu_{2}}{c^{\prime}} J_{n+1}\left(\alpha_{m} c^{\prime}\right)-\frac{2 n(n-1) \mu_{2}}{c^{\prime 2}} J_{n}\left(\alpha_{m} c^{\prime}\right)\right] A_{m n}^{(2)}} \\
& +\left[\frac{2 n \alpha \mu_{2}}{c^{\prime}} Y_{n+1}\left(\alpha_{m} c^{\prime}\right)-\frac{2 n(n-1) \mu_{2}}{c^{\prime 2}} Y_{n}\left(\alpha_{m} c^{\prime}\right)\right] B_{m n}^{(2)} \\
& +\left[-\frac{\beta_{m} \mu_{2}}{c^{\prime}} J_{n+1}\left(\beta_{m} c^{\prime}\right)+\left(\beta_{m}^{2} \mu_{2}-\frac{2 n(n-1) \mu_{2}}{c^{\prime 2}}\right) J_{n}\left(\beta_{m} c^{\prime}\right)\right] C_{m n}^{(2)} \\
& +\left[-\frac{\beta_{m} \mu_{2}}{c^{\prime}} Y_{n+1}\left(\beta_{m} c^{\prime}\right)+\left(\beta_{m}^{2} \mu_{2}-\frac{2 n(n-1) \mu_{2}}{c^{\prime 2}}\right) Y_{n}\left(\beta_{m} c^{\prime}\right)\right] D_{m n}^{(2)} \\
& +\left[-\frac{2(n+1) i k_{m} \mu_{2}}{c^{\prime}} J_{n+1}\left(\beta_{m} c^{\prime}\right)+i k_{m} \beta_{m} \mu_{2} J_{n}\left(\beta_{m} c^{\prime}\right)\right] E_{m n}^{(2)} \\
& +\left[-\frac{2(n+1) i k_{m} \mu_{2}}{c^{\prime}} Y_{n+1}\left(\beta_{m} c^{\prime}\right)+i k_{m} \beta_{m} \mu_{2} Y_{n}\left(\beta_{m} c^{\prime}\right)\right] F_{m n}^{(2)}=0
\end{aligned}
$$




$$
\begin{gathered}
\quad\left[-2 i k_{m} \alpha_{m} J_{n+1}\left(\alpha_{m} c^{\prime}\right)+\frac{2 n i k_{m} \mu_{2}}{c^{\prime}} J_{n}\left(\alpha_{m} c^{\prime}\right)\right] A_{m n}^{(2)} \\
+\left[-2 i k_{m} \alpha_{m} Y_{n+1}\left(\alpha_{m} c^{\prime}\right)+\frac{2 n i k_{m} \mu_{2}}{c^{\prime}} Y_{n}\left(\alpha_{m} c^{\prime}\right)\right] B_{m n}^{(2)} \\
+\left[\frac{n i k_{m} \mu_{2}}{c^{\prime}} J_{n}\left(\beta_{m} c^{\prime}\right)\right] C_{m n}^{(2)}+\left[\frac{n i k_{m} \mu_{2}}{c^{\prime}} Y_{n}\left(\beta_{m} c^{\prime}\right)\right] D_{m n}^{(2)} \\
+\left[\mu_{2}\left(\beta_{m}^{2}-k_{m}^{2}\right) J_{n+1}\left(\beta_{m} c^{\prime}\right)-\frac{n \beta_{m} \mu_{2}}{c^{\prime}} J_{n}\left(\beta_{m} c^{\prime}\right)\right] E_{m n}^{(2)} \\
+\left[\mu_{2}\left(\beta_{m}^{2}-k_{m}^{2}\right) Y_{n+1}\left(\beta_{m} c^{\prime}\right)-\frac{n \beta_{m} \mu_{2}}{c^{\prime}} Y_{n}\left(\beta_{m} c^{\prime}\right)\right] F_{m n}^{(2)}=0
\end{gathered}
$$

For each $(m, n)$ index, Equations 4.4.41 - 4.4.52 are grouped together to form a 12dimensional matrix equation, with unknown wave coefficients are grouped together into a vector, $\mathbf{x}$

$$
\boldsymbol{A}_{m n} \boldsymbol{x}_{m n}=-\frac{1}{a b} \sum_{s=-\infty}^{\infty} \boldsymbol{Z}_{m-s} \boldsymbol{x}_{s n}-\frac{1}{a b} \sum_{s=-\infty}^{\infty} \boldsymbol{T}_{m-s} \boldsymbol{x}_{s n}-\frac{1}{a b} \sum_{s=-\infty}^{\infty} \boldsymbol{R}_{m-s} \boldsymbol{x}_{s n}+\boldsymbol{f} \delta_{m 0}
$$

$A_{m n}$ is a $12 \times 12$ matrix that describes the shell dynamics for the $(m, n)$ mode in which the rows represent the 12 boundary conditions and the columns are multiplied with the 12 -element vector of unknown coefficients, $\mathbf{x}_{\mathrm{mn}}$

$$
\boldsymbol{x}_{m n}=\left[A_{m n}^{(1)}, B_{m n}^{(1)}, C_{m n}^{(1)}, D_{m n}^{(1)}, E_{m n}^{(1)}, F_{m n}^{(1)}, A_{m n}^{(2)}, B_{m n}^{(2)}, C_{m n}^{(2)}, D_{m n}^{(2)}, E_{m n}^{(2)}, F_{m n}^{(2)}\right]^{T}
$$

For this system, the $12 \times 12$ stiffener matrices $\left(\mathrm{Rm}_{\mathrm{m}-\mathrm{s}}, \mathrm{T}_{\mathrm{m}-\mathrm{s}}, \mathrm{Zm}-\mathrm{s}\right)$ are mostly zeros with the nonzero elements given by 4.2.18 - 4.2.20, and external excitation $\mathrm{f}$ was presented previously as 4.3.19 for the fluid plane wave. Each $(\mathrm{m}, \mathrm{n})$ mode of 4.4 .53 is grouped into a global matrix equation of circumferential modes, $n$,

$$
\widehat{A}_{\boldsymbol{n}} \widehat{\boldsymbol{x}}_{\boldsymbol{n}}=-\frac{1}{a b} \widehat{\boldsymbol{Z}}_{\boldsymbol{n}} \widehat{\boldsymbol{x}}_{n}-\frac{1}{a b} \widehat{\boldsymbol{T}}_{\boldsymbol{n}} \widehat{\boldsymbol{x}}_{n}-\frac{1}{a b} \widehat{\boldsymbol{R}}_{\boldsymbol{n}} \widehat{\boldsymbol{x}}_{\boldsymbol{n}}+\widehat{\boldsymbol{f}}_{\boldsymbol{n}}
$$

where each component has the exact structure of 2.4.46. To determine the n-mode vector of coefficients $\hat{\mathbf{x}}_{\mathbf{n}}, 4.4 .55$ must be solved.

$$
\widehat{\boldsymbol{x}}_{\boldsymbol{n}}=\left[\widehat{\boldsymbol{A}}_{\boldsymbol{n}}+\frac{1}{a b} \widehat{\boldsymbol{Z}}_{\boldsymbol{n}}+\frac{1}{a b} \widehat{\boldsymbol{T}}_{\boldsymbol{n}}+\frac{1}{a b} \widehat{\boldsymbol{R}}_{\boldsymbol{n}}\right]^{-1} \widehat{\boldsymbol{f}}_{\boldsymbol{n}}
$$

Finally, the solution of 4.4 .56 for each n-mode is used with $4.4 .3-4.4 .8$ to construct the displacement responses of the entire shell and coating.

\subsubsection{Model Validation}

Comparing the coated elastic system with the reference thin-shell model is a bit more complicated than with the prior models. The thin-shell formulation can't support additional bodies, so the coated elastic model must be configured to match a solid single shell. Using steel 
material properties for the coating and setting the thicknesses to be equal and half of the reference shell, allows the new model to be validated. Parameters used for validation are displayed in Table 4.4.1.

\begin{tabular}{|c|c|c|c|}
\hline Property & Symbol & Value & Units \\
\hline Young's Modulus & $\mathrm{E}$ & $210 \times 10^{9}$ & $\mathrm{~N} / \mathrm{m} 2$ \\
\hline Poisson's Ratio & $v$ & 0.30 & None \\
\hline Structural Damping & $\eta$ & 0.00 & None \\
\hline Density & $\rho$ & 7850 & $\mathrm{~kg} / \mathrm{m} 3$ \\
\hline Lamé 1st Parameter & $\lambda$ & $1.212 \times 10^{11}$ & $\mathrm{~N} / \mathrm{m} 2$ \\
\hline Shear Modulus & $\mu, \mathrm{G}$ & $8.077 \times 10^{10}$ & $\mathrm{~N} / \mathrm{m} 2$ \\
\hline Thickness (Shell) & $\mathrm{h}$ & 0.0005 & $\mathrm{~m}$ \\
\hline Thickness (Coating) & $\mathrm{h}_{\mathrm{c}}$ & 0.0005 & $\mathrm{~m}$ \\
\hline
\end{tabular}

Table 4.4.1 - Material and Geometry Properties for Shell and Coating

The combined thickness of the new model is $h=0.001 \mathrm{~m}$, and the material properties are all identical; rib material is steel and the same as above.

Following the validation procedure from the prior models, both a ring loading and acoustic wave are used. Applying a ring loading with zero wavenumber $(k=0)$ at low frequency excites the new and reference systems as shown in Figures 4.4.3 and 4.4.4. 

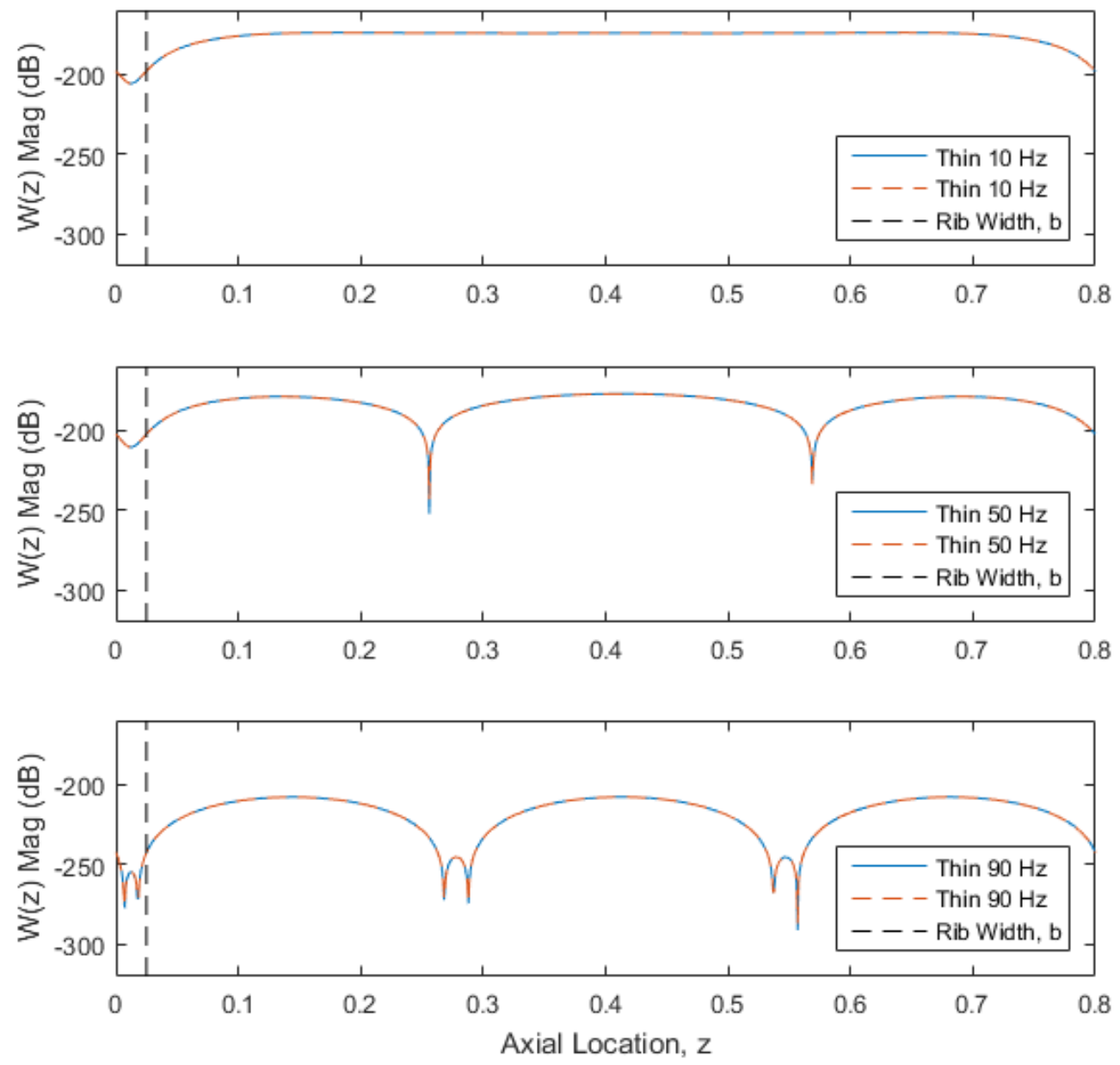

Figure 4.4.3 - Comparison of radial displacement of reference and coated-elastic system excited by 10,50 and $90 \mathrm{~Hz}$ Ring Loading $(\mathrm{k}=0), \mathrm{m}_{\mathrm{pts}}=31$ 

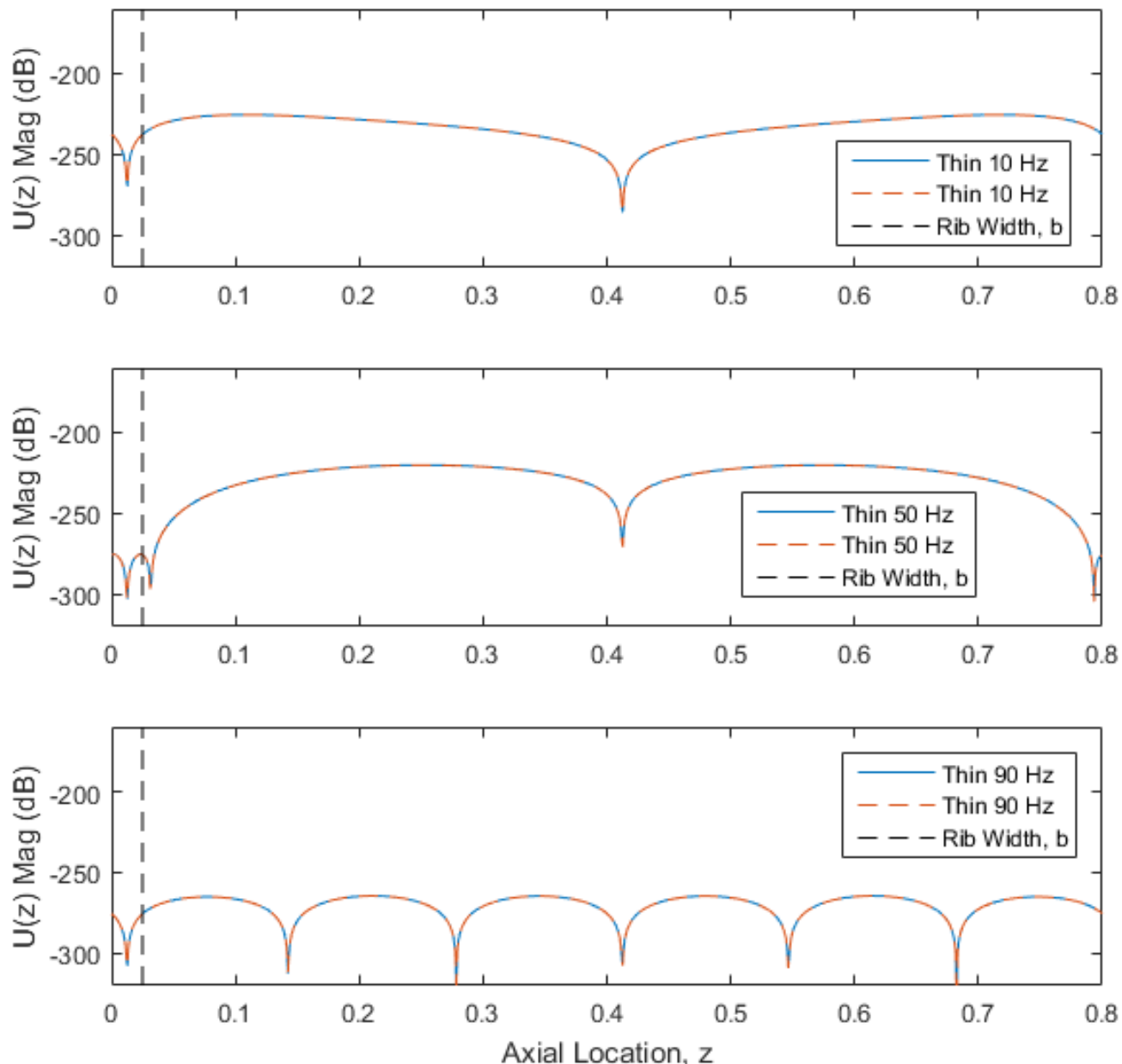

Figure 4.4.4 - Comparison of axial displacement of reference and coated-elastic system excited by 10,50 and $90 \mathrm{~Hz}$ Ring Loading $(\mathrm{k}=0), \mathrm{m}_{\mathrm{pts}}=31$

It is important that the radius of the output displacement fields matches that of the reference model, because the elastic outputs vary significantly across the thickness. The elastic model has been configured to output responses at the nominal shell radius, $r=4.5 \mathrm{~m}$, which is the nominal radius of the thin-shell system. The elastic model matches the reference quite well. The acoustic wave response is shown in Figure 4.4.5. 

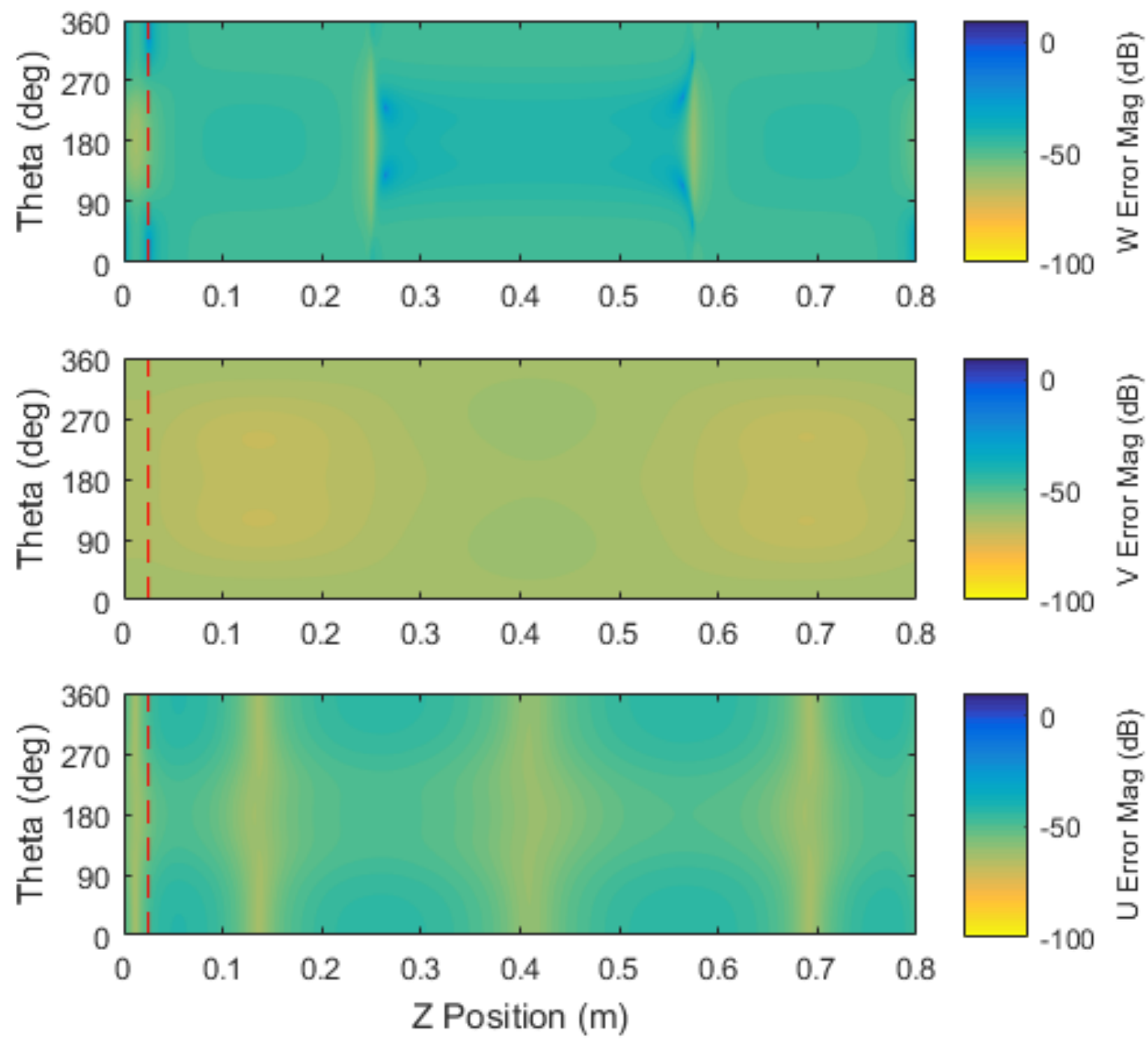

Figure 4.4.5 - Thin and Thick-shell radial (top), tangential (mid), and axial (bottom) Normalized Error in dB for plane-wave: $50 \mathrm{~Hz}, \varphi_{\mathrm{i}}=\pi / 12$, mpts $=31$, npts $=7$. Rib is located between the left edge $(\mathrm{z}=0)$ and the red dashed line.

Normalized errors between the reference thin-shell model (with fluid-loading) and the coated elastic model are less than $-40 \mathrm{~dB}$ throughout each displacement field, representing less than $1 \%$ error. There are 4 nodes within the radial displacement response which cause artificially high errors along the slice $\theta=130 \mathrm{deg}$ and $\theta=230 \mathrm{deg}$. Refer to Figure 4.3.6 for a plot of the response along these slices. It is a good idea to check the convergence of the coated validation model, and thus the normalized magnitudes of the displacement coefficients are shown for each axial and circumferential mode. 

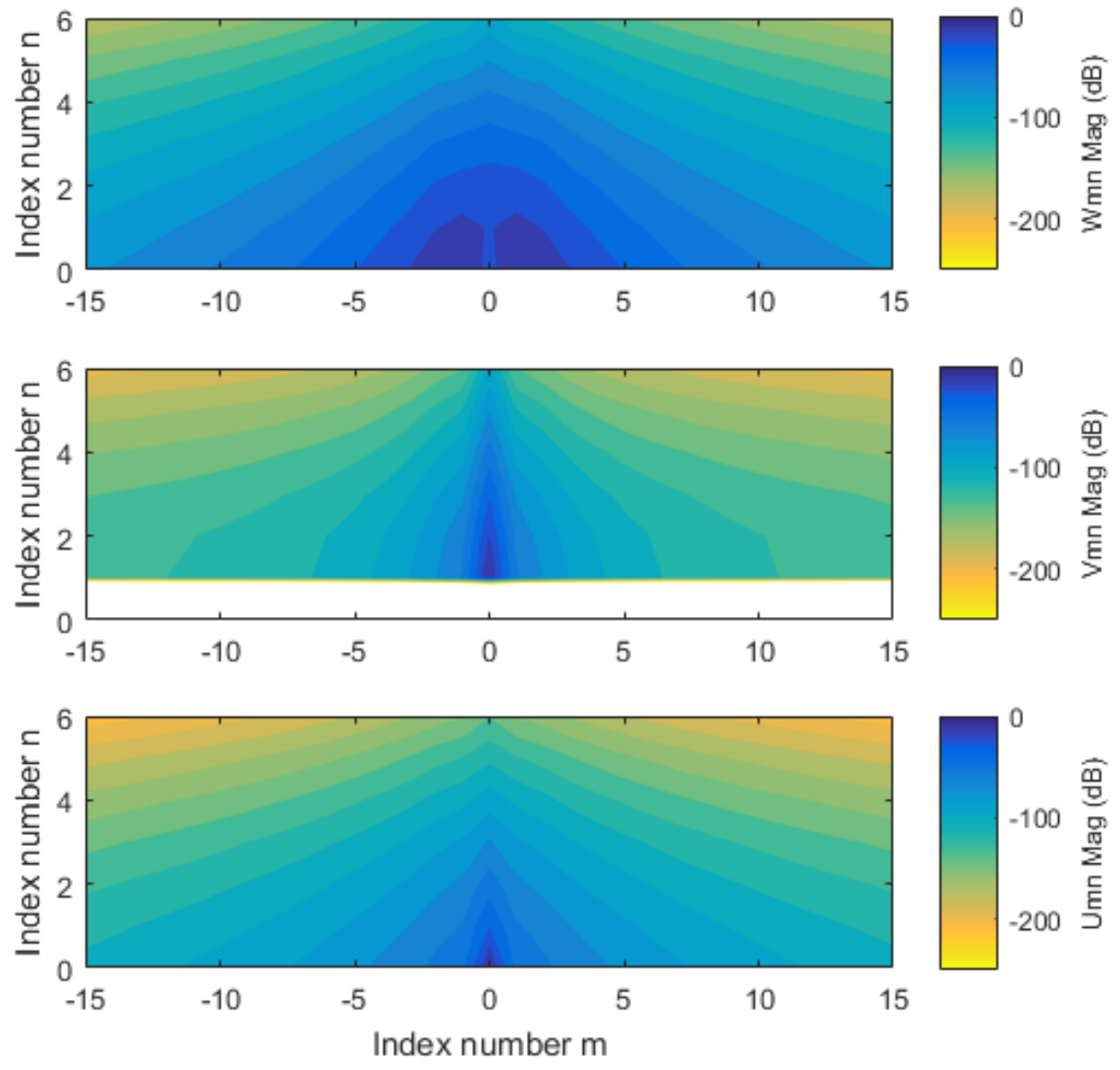

Figure 4.4.6 - Normalized magnitude of displacement modes for each circumferential index, $n$ and axial index, $m$

As seen with the base model (Figure 3.1.25), the largest modes are located at $m=0, n=0$, and the magnitudes have dropped considerably at the outer edges, indicating that the simulation has converged. Finally, the scattered pressure errors are displayed. 

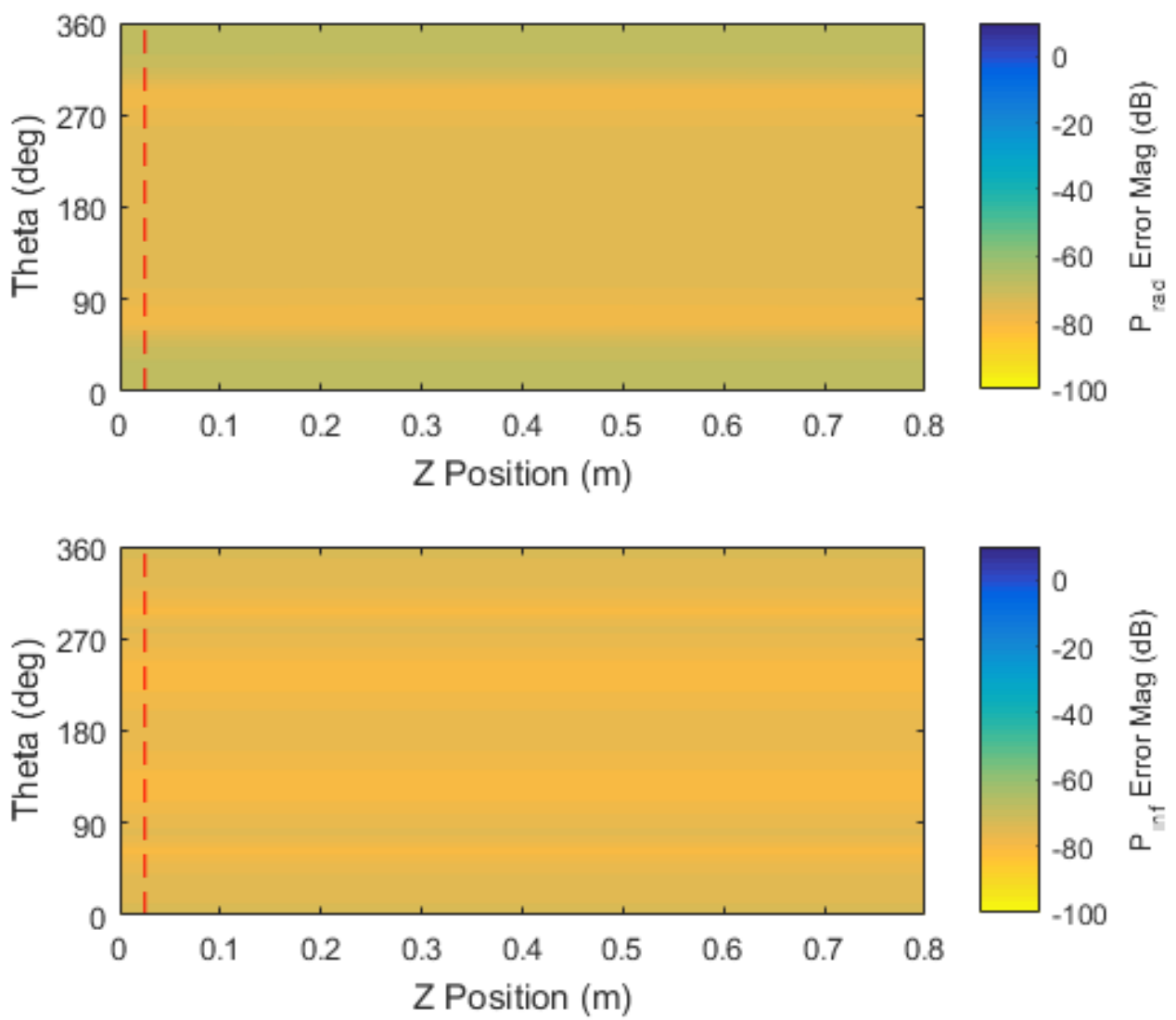

Figure 4.4.7 - Thin and Thick-shell radiated pressure (top) and reflected pressure (bottom) Normalized Error in $\mathrm{dB}$ for plane-wave: $50 \mathrm{~Hz}, \varphi_{\mathrm{i}}=\pi / 12$, mpts $=31$, npts $=7$. Rib is located between the left edge $(\mathrm{z}=0)$ and the red dashed line.

Scattered pressure error values are at least $-70 \mathrm{~dB}$, less than $0.05 \%$. Considering this, the coated elastic model with fluid-loading and Timoshenko beam-ribs of finite width is considered to be validated. 


\section{Chapter 5 Coated Elastic Shell Results}

Combining each of the features described in Chapter 4, Model 5 adds an acoustic coating, represented as a solid elastic body. Coating material properties are quite different from the steel shell, and are portrayed below. The system is excited by ring load and acoustic wave inputs, and the resultant displacement and pressure fields are described. Backscatter pressure is compared to the fluid-loaded Model 4.

\subsection{Final Model Results}

\subsubsection{Material and Geometric Properties}

With the validation of each model fulfilled, it is time to discuss the behavior of the coated elastic fluid-loaded beam-rib model with real parameters. The shell and rib materials are the same as the base model, but the coating now represents a urethane polymer, and has a much larger thickness than before. Tables 5.1.1 display the material properties for the coating subsystem. 


\begin{tabular}{|c|c|c|c|}
\hline Property & Symbol & Value & Units \\
\hline Young's Modulus & $\mathrm{E}$ & $158 \times 10^{6}$ & $\mathrm{~N} / \mathrm{m}^{2}$ \\
\hline Poisson's Ratio & $\nu$ & 0.487 & None \\
\hline Structural Damping $(\lambda)$ & $\eta^{1}$ & 0.01 & None \\
\hline Structural Damping $(\mu)$ & $\eta_{2}$ & 0.10 & None \\
\hline Density & $\rho$ & 1100 & $\mathrm{~kg} / \mathrm{m}^{3}$ \\
\hline Lamé 1st Parameter & $\lambda$ & $2.11 \times 10^{9}$ & $\mathrm{~N} / \mathrm{m}^{2}$ \\
\hline Shear Modulus & $\mu, \mathrm{G}$ & $53.2 \times 10^{6}$ & $\mathrm{~N} / \mathrm{m}^{2}$ \\
\hline
\end{tabular}

Table 5.1.1 - Material Properties for Coating

Urethane is obviously much softer and less dense than steel, but it also has significant structural damping. Structural damping is handled differently compared to steel, because it is distinct for dilatational waves and shear waves. Equation 5.1.1 and 5.1.2 give new modified expressions for $\lambda$ and $\mu$ for the coating

$$
\begin{aligned}
& \lambda_{c}=\lambda\left(1-i \eta_{1}\right) \\
& \mu_{c}=\mu\left(1-i \eta_{2}\right)
\end{aligned}
$$

while the coating Young's Modulus is simply the nominal value given in Table 5.1.1. Likewise, the geometry of the coating is far different than that used for validation.

\begin{tabular}{|c|c|c|c|}
\hline Property & Symbol & Value & Units \\
\hline Inner Radius & $\mathrm{c}$ & 4.5254 & $\mathrm{~m}$ \\
\hline Thickness & $\mathrm{h}_{\mathrm{c}}$ & 0.0762 & $\mathrm{~m}$ \\
\hline Outer Radius & $\mathrm{c}^{\prime}$ & 4.6016 & $\mathrm{~m}$ \\
\hline
\end{tabular}

Table 5.1.2 - Dimensional Properties for Coating

Coating thickness is 3 times that of the shell thickness, leading to an overall system thickness of $0.1016 \mathrm{~m}$.

\subsubsection{Ring Loading}

With the parameters established, the results from both types of excitations can be investigated. There is no need to check the convergence for these runs; the validation simulation has a much more aggressive response due to the soft shell, and thus requires more modes to achieve sufficient results. Applying the ring load with zero wavenumber results in radial and axial displacement fields at the outer coating surface shown in Figures 5.1.1 and 5.1.2. 


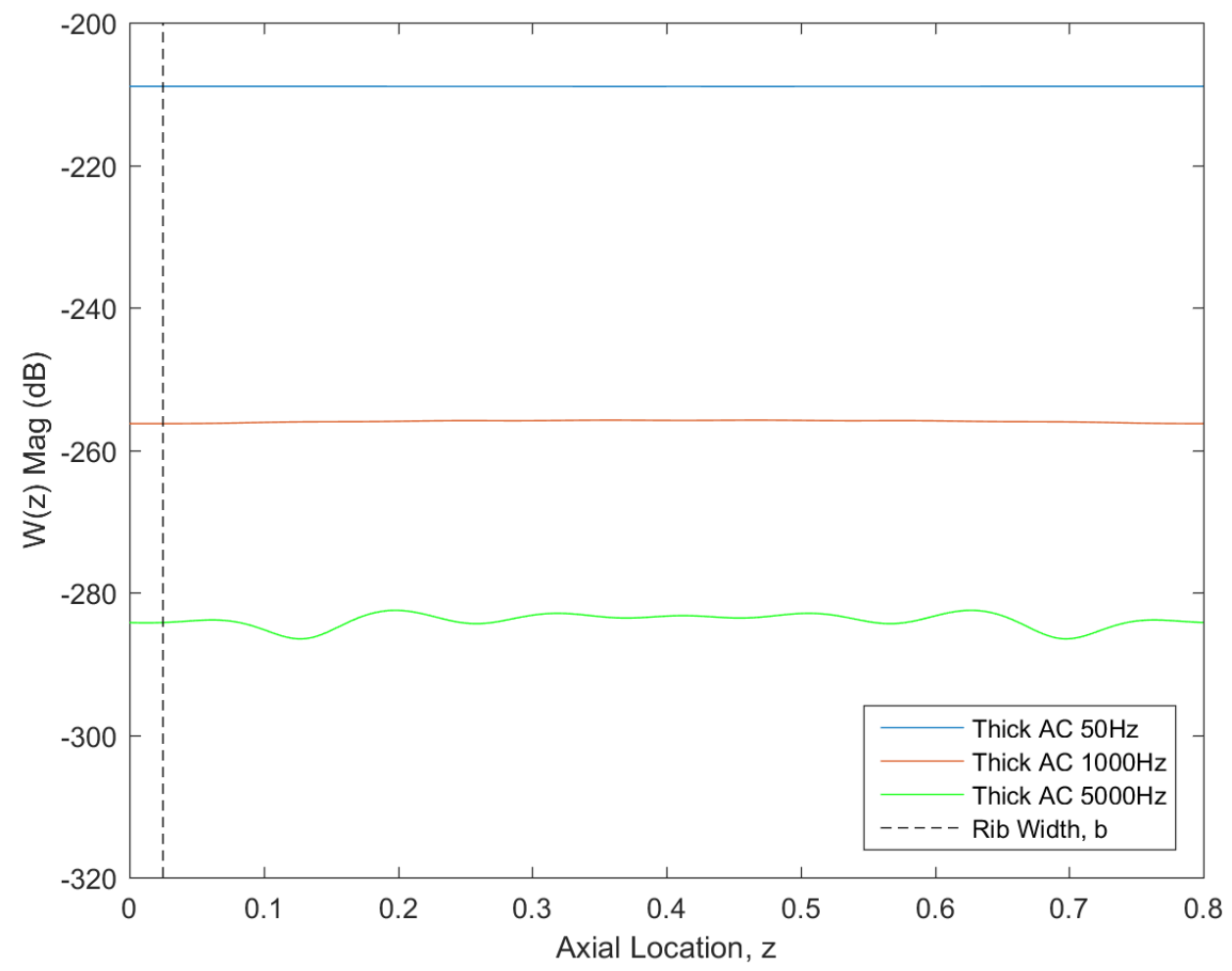

Figure 5.1.1 - Radial surface displacement response of coated elastic model excited by ring loading $(\mathrm{k}=0)$ at $50,1000,5000 \mathrm{~Hz}$

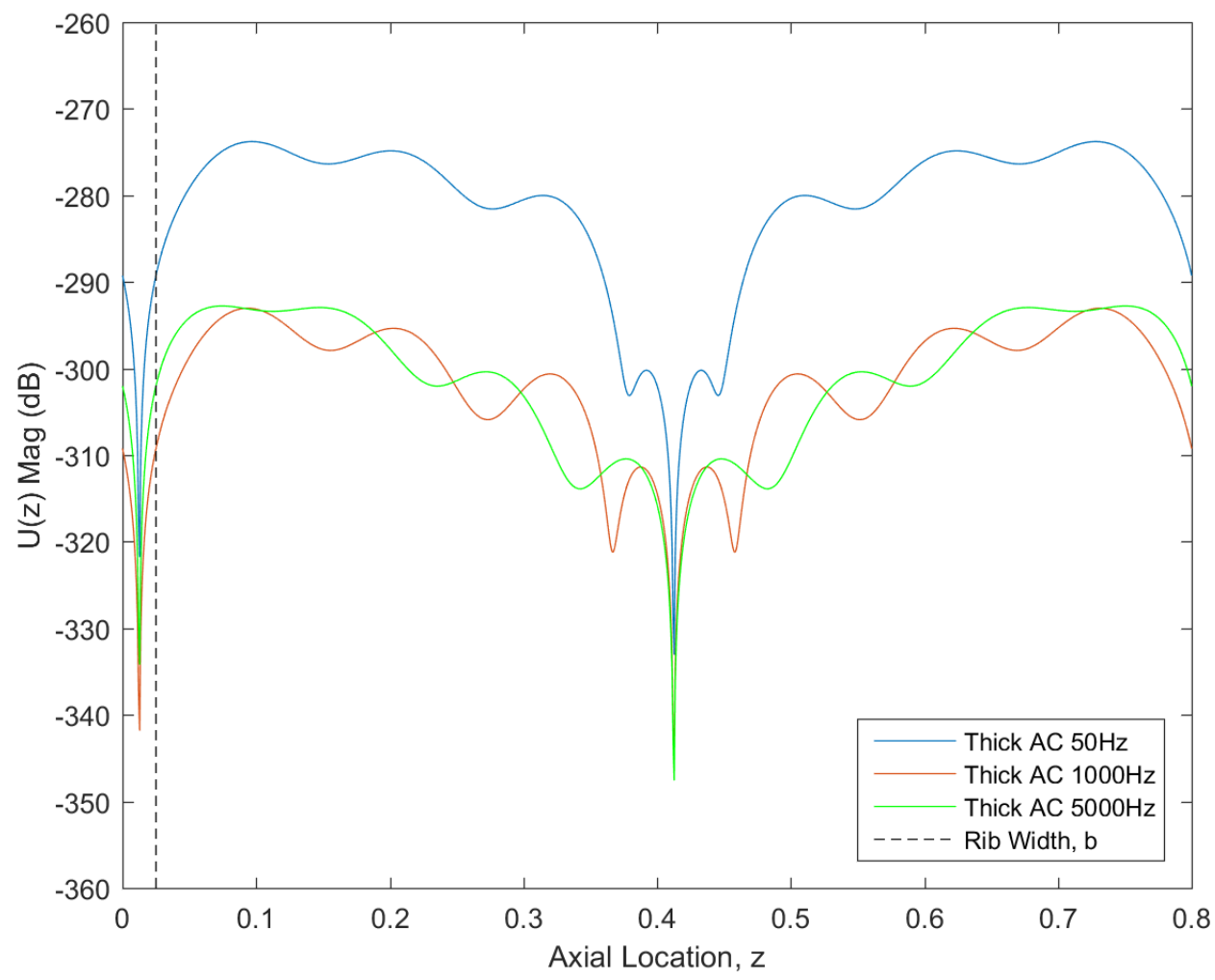

Figure 5.1.2 - Axial surface displacement response of coated elastic model excited by ring loading $(\mathrm{k}=0)$ at $50,1000,5000 \mathrm{~Hz}$ 
As expected the response is symmetric inside the rib region, and also outside. There is much spatial variation in the axial response. Amplitudes are certainly less than the base model (Figures 3.1.26 and 3.1.27), but this expected due to the effects of the external fluid.

The coating has an effect on the attenuation of waves closer to the surface, but this is hard to see in a single trajectory. Figure 5.1.3 displays a contour of the radial displacement through the thickness.

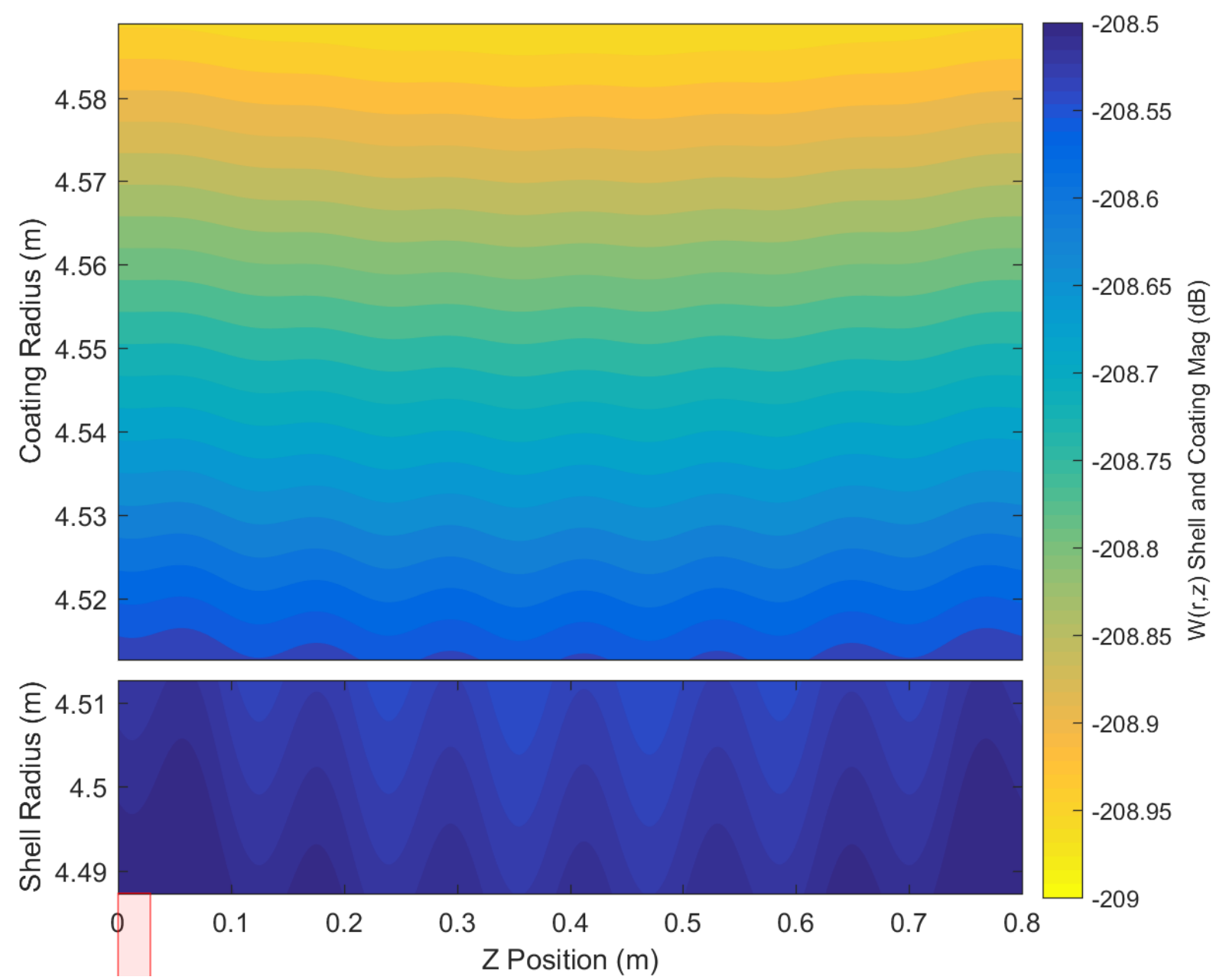

Figure 5.1.3 - Radial displacement field of coated system for cross-section through the cylinder thickness at angle $\theta=0$, for ring loading $(\mathrm{k}=0, \mathrm{f}=50 \mathrm{~Hz})$; red lines are the rib

The figure above can be thought of as a slice through the center of the cylinder along the $z$ axis; it cuts the system into 2 halves and the upper half is shown, with the space between the plots representing the shared interface. The coating thickness is $3 x$ larger than the shell thickness, so the two subplots are to relative scale. Since the ring loading is symmetric, the other angular positions are identical. Though the scale is small ( $0.5 \mathrm{~dB}$ total), the differences is roughly $6 \%$ from the lower surface at $r=4.48725 \mathrm{~m}$ to the upper surface at $r=4.5127 \mathrm{~m}$. However, looking at the axial displacement field from the same slice shows a very interesting displacement response shown in Figure 5.1.4. 


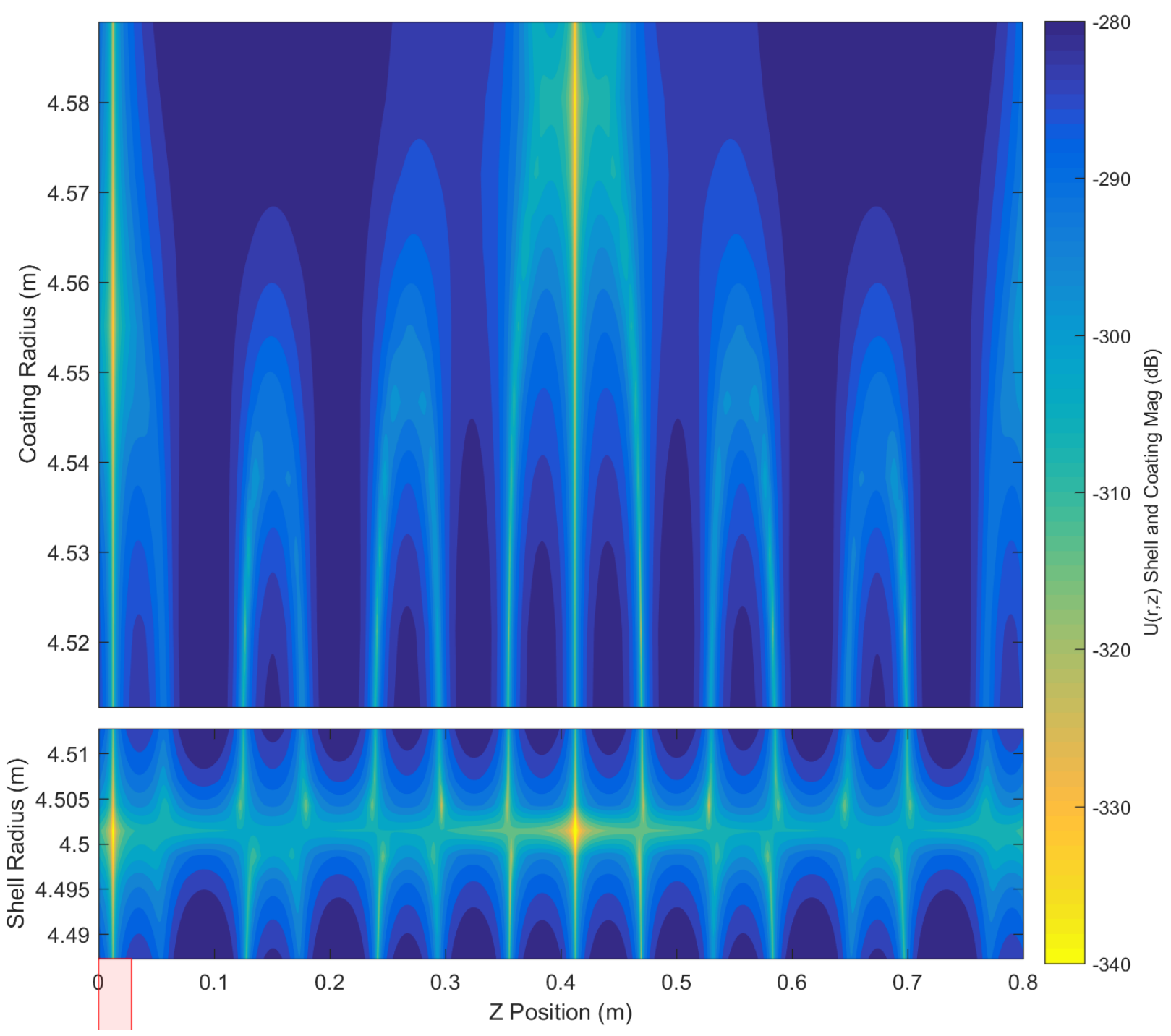

Figure 5.1.4 - Axial displacement field of coated system for cross-section through the cylinder thickness at angle $\theta=0$, for ring loading $(\mathrm{k}=0, \mathrm{f}=50 \mathrm{~Hz})$.

Note the large difference in scale between Figure 5.1.3 and 5.1.4, both the average value and the range in each plot. The radial displacement field is mostly uniform across the thickness, with some slight variation, but the axial response shows a distinct effect from the single stiffener $(0 \leq$ $z \leq 0.025)$. The rib is located only at the inner shell surface $(r=4.4875 \mathrm{~m})$, and is represented by the red rectangle at $z=0$. There is actually less axial attenuation at the coating outer surface $(r=$ $4.589 \mathrm{~m}$ ), while the smallest magnitude is found within the stiffener region, as expected. The vertical lines are areas of low displacement, and the middle of the shell $(r=4.5 \mathrm{~m})$ deforms less than any other radius in the system. At $5000 \mathrm{~Hz}$, the ring load creates a similar response $(1000 \mathrm{~Hz}$ response not shown). 


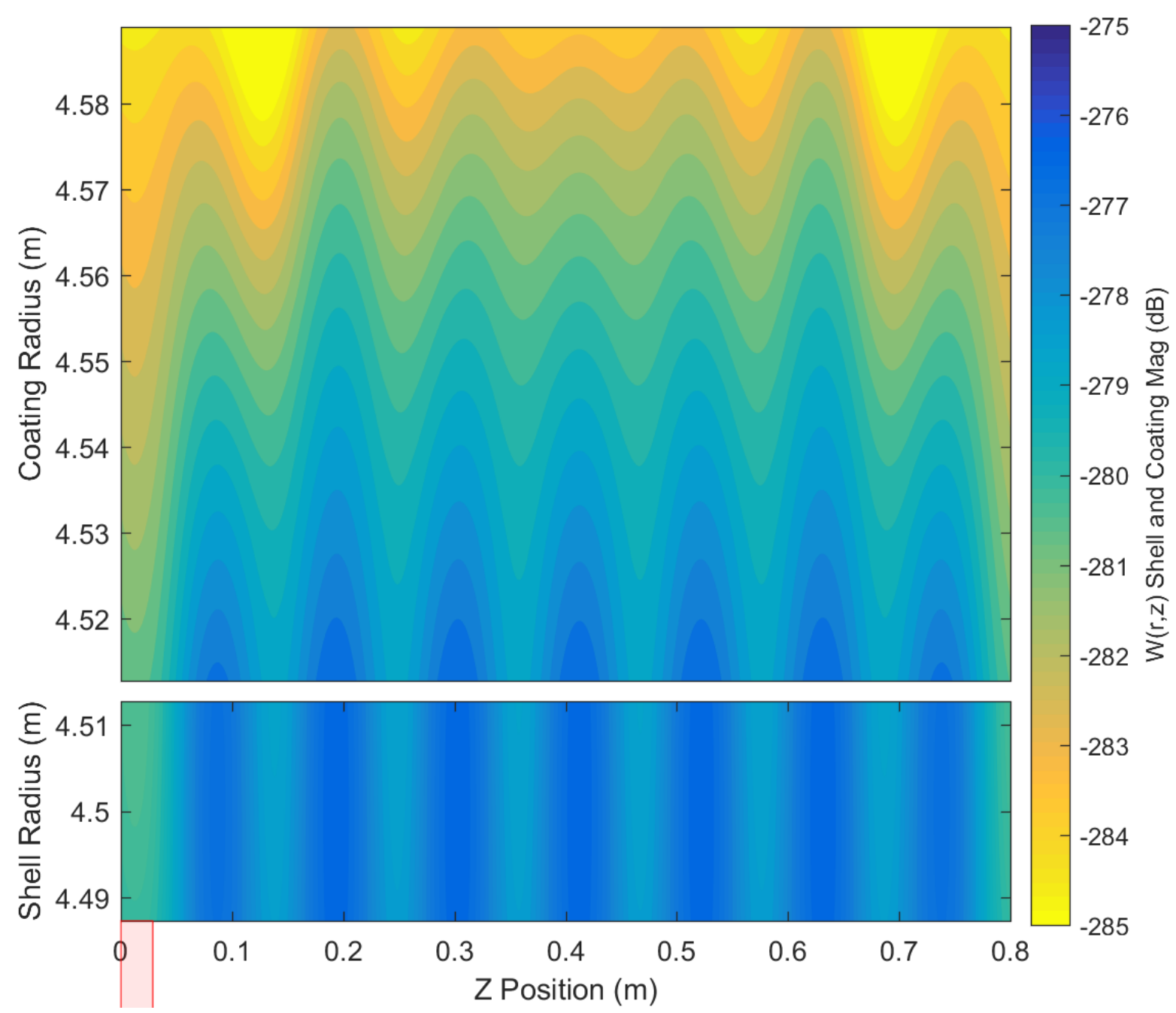

Figure 5.1.5 - Radial displacement field of coated system for cross-section through the cylinder thickness at angle $\theta=0$, for ring loading $(\mathrm{k}=0, \mathrm{f}=5000 \mathrm{~Hz})$

Magnitudes are more attenuated, but there is more radial variation as seen by the larger $\mathrm{dB}$ range required. Vertical lines of low deformation are now showing, where the displacement field near the rib is being repeated through the cylinder length. 


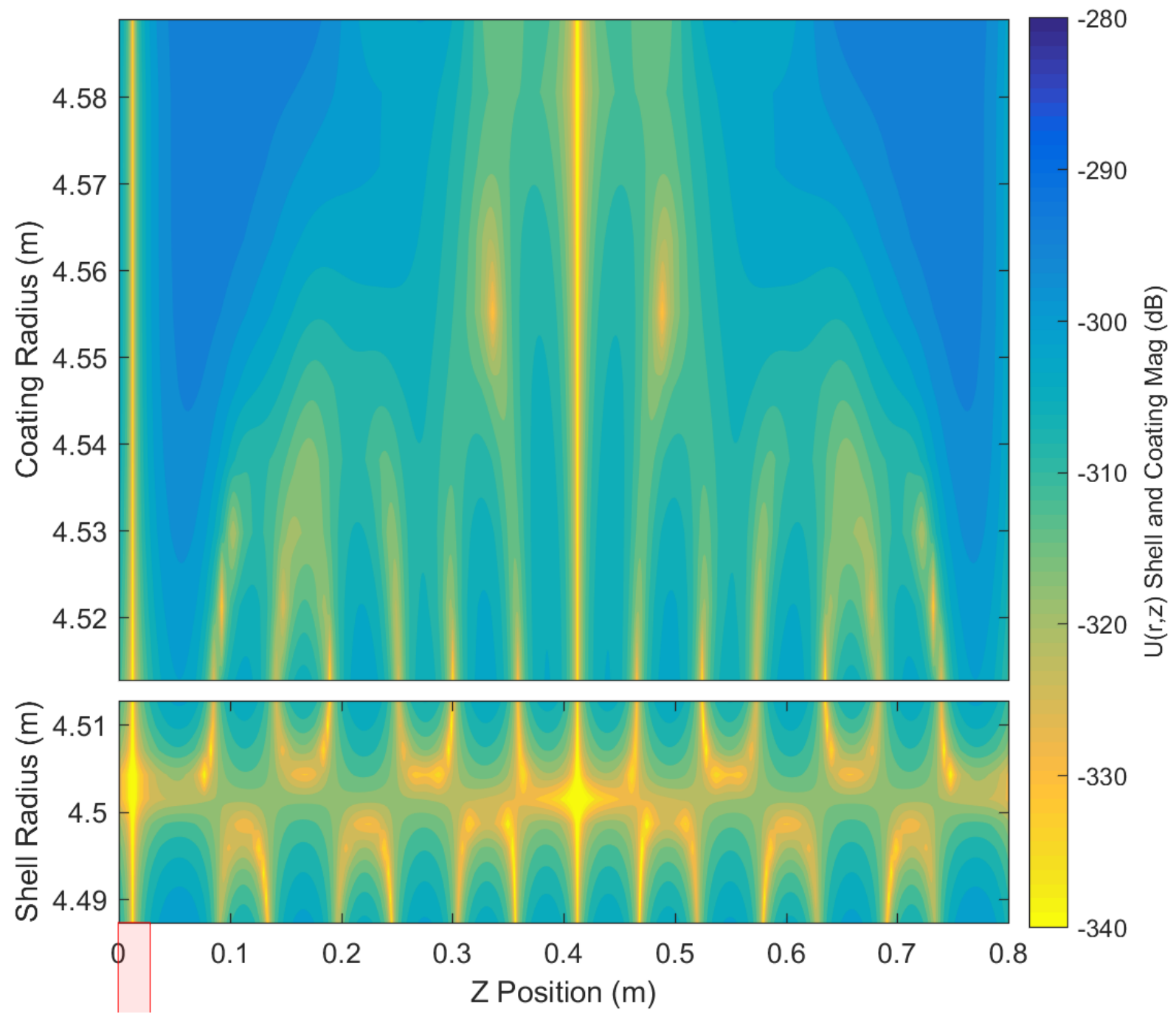

Figure 5.1.6 - Axial displacement field of coated system for cross-section through the cylinder thickness at angle $\theta=0$, for ring loading $(\mathrm{k}=0, \mathrm{f}=5000 \mathrm{~Hz})$

Using the same scale as Figure 5.1.4, the overall amplitude has lowered compared to the low frequency response. However, the same overall shape is present, with relatively little motion occurring within the rib section.

\subsubsection{Acoustic Wave}

An incoming plane wave, with angle of incidence of 15 degrees from broadside, impacts the outer coating surface. In terms of accessing performance, both the displacement response of the system and the scattered radiation are important. First the displacement fields of the outer coating surface are shown in Figures 5.1.7 - 5.1.9. 

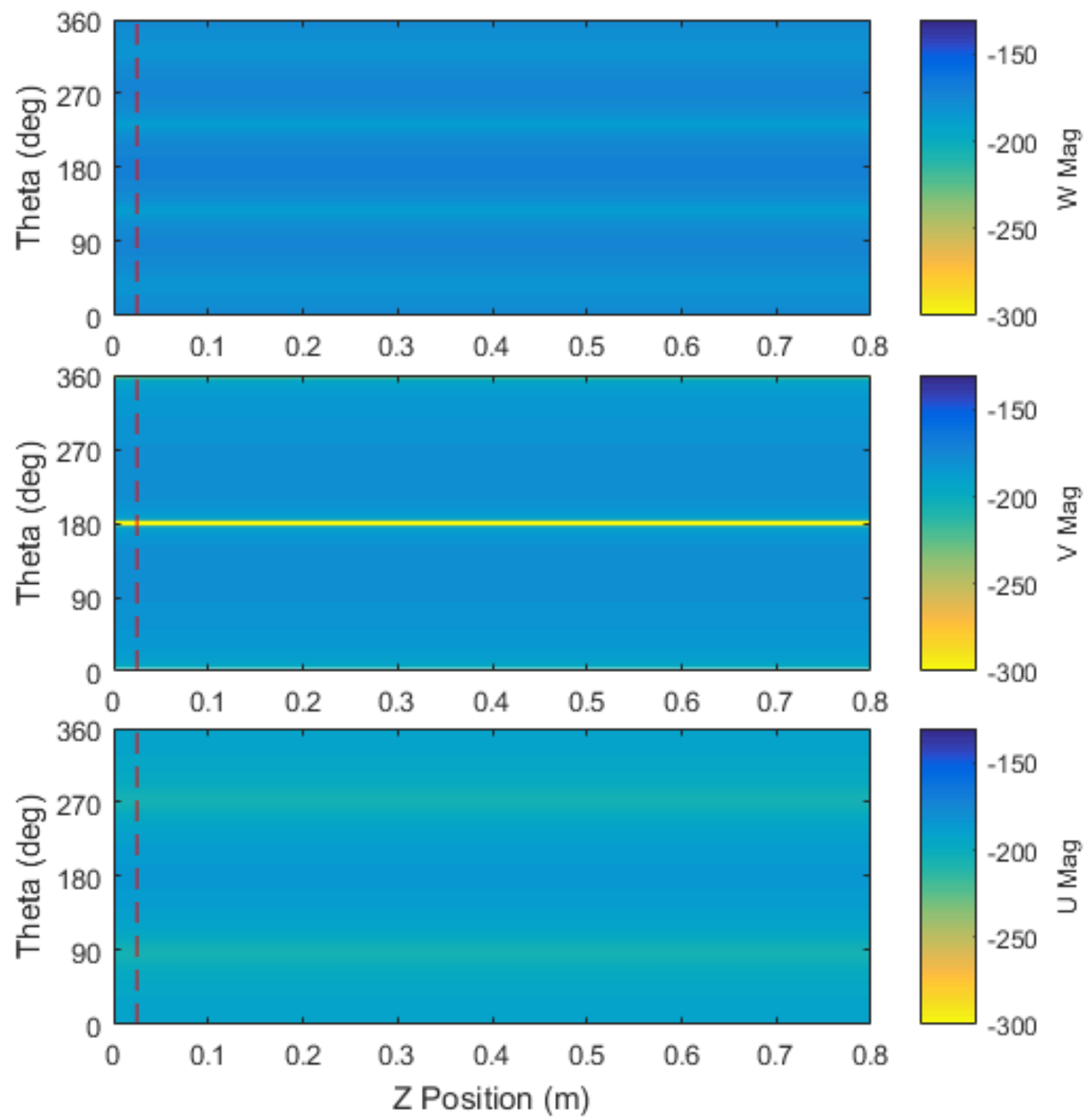

Figure 5.1.7 - Coating surface radial (top), tangential (mid), and axial (bottom) displacement response for acoustic-wave: $50 \mathrm{~Hz}, \varphi \mathrm{i}=15 \mathrm{deg}$, mpts $=31$, npts $=7$ 


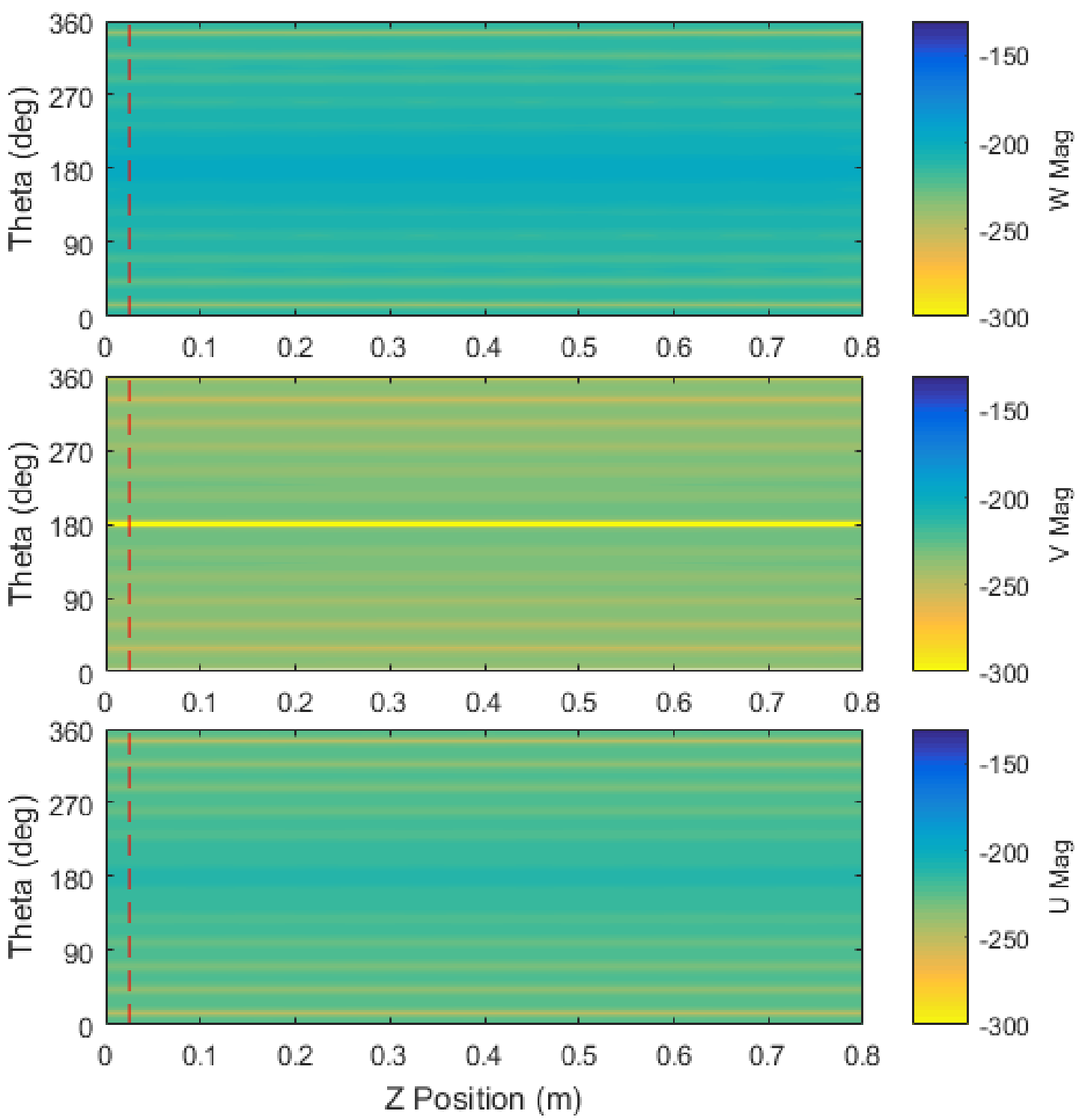

Figure 5.1.8 - Coating surface radial (top), tangential (mid), and axial (bottom) displacement response for acoustic-wave: $1000 \mathrm{~Hz}, \varphi \mathrm{i}=15 \mathrm{deg}$, $\mathrm{mpts}=31$, npts $=7$ 


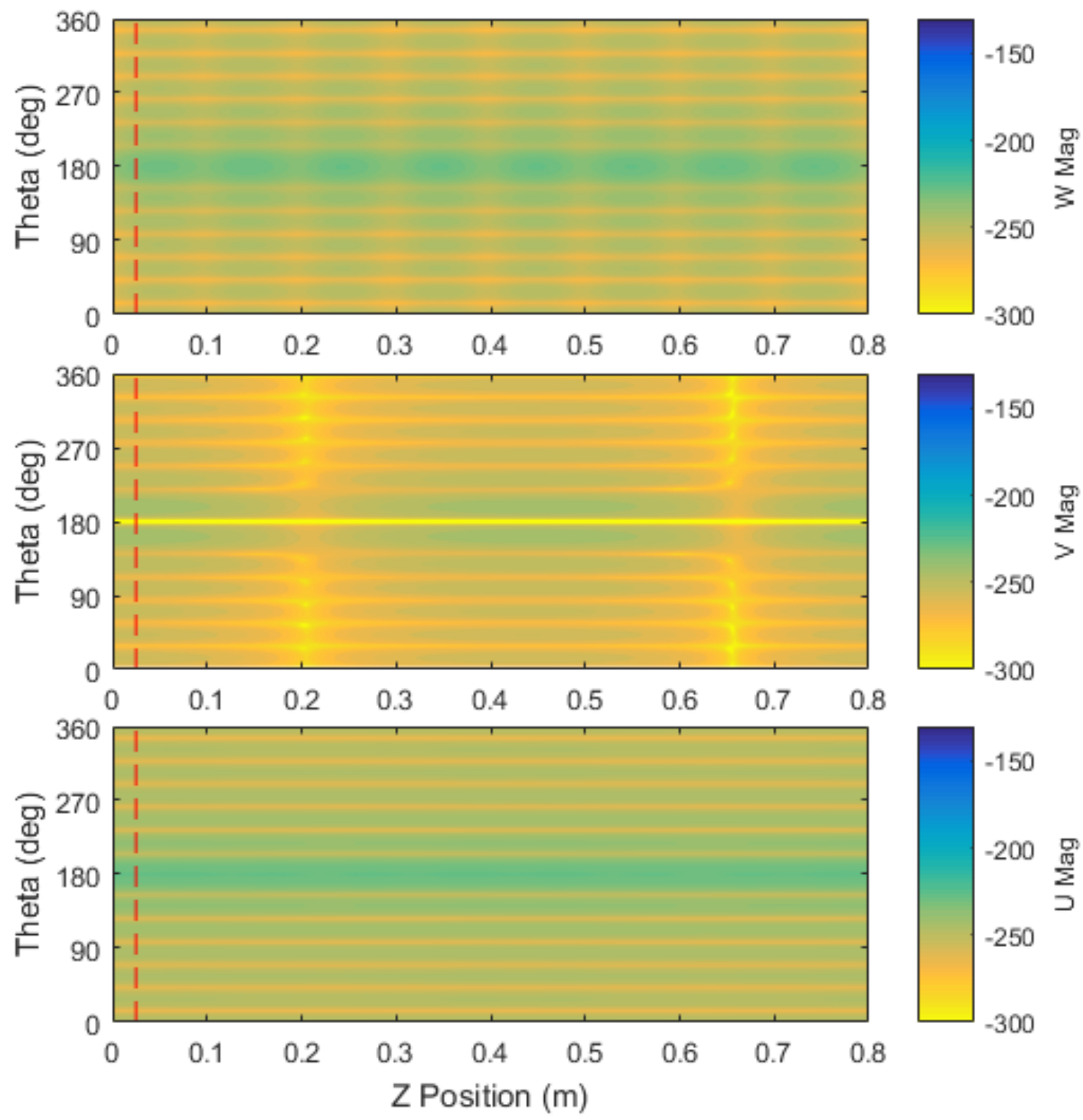

Figure 5.1.9 - Coating surface radial (top), tangential (mid), and axial (bottom) displacement response for acoustic-wave: $5000 \mathrm{~Hz}, \varphi \mathrm{i}=15 \mathrm{deg}$, $\mathrm{mpts}=31$, npts $=7$

Circumferential spatial waves dominate the responses at all excitation frequencies; at $50 \mathrm{~Hz}$ there is a low order mode present, while at $1-5 \mathrm{kHz}$ the same higher order circumferential dilatational and shear modes dominate. Vertical bands begin to show up at $5 \mathrm{kHz}$ in the radial and tangential responses, and the axial shear mode is of higher order than seen in the base model (Figure 3.1.30).

Taking a "slice" along the radius provides a view of the displacement variation within the thickness, analogous to Figures 5.1.3 - 5.1.6. With the acoustic input, the response is not symmetric along the circumferential direction; thus each angular position will produce a slice with a different contour. The $5000 \mathrm{~Hz}$ response shows interesting behavior around $\theta=10 \mathrm{deg}$ and is shown below. 


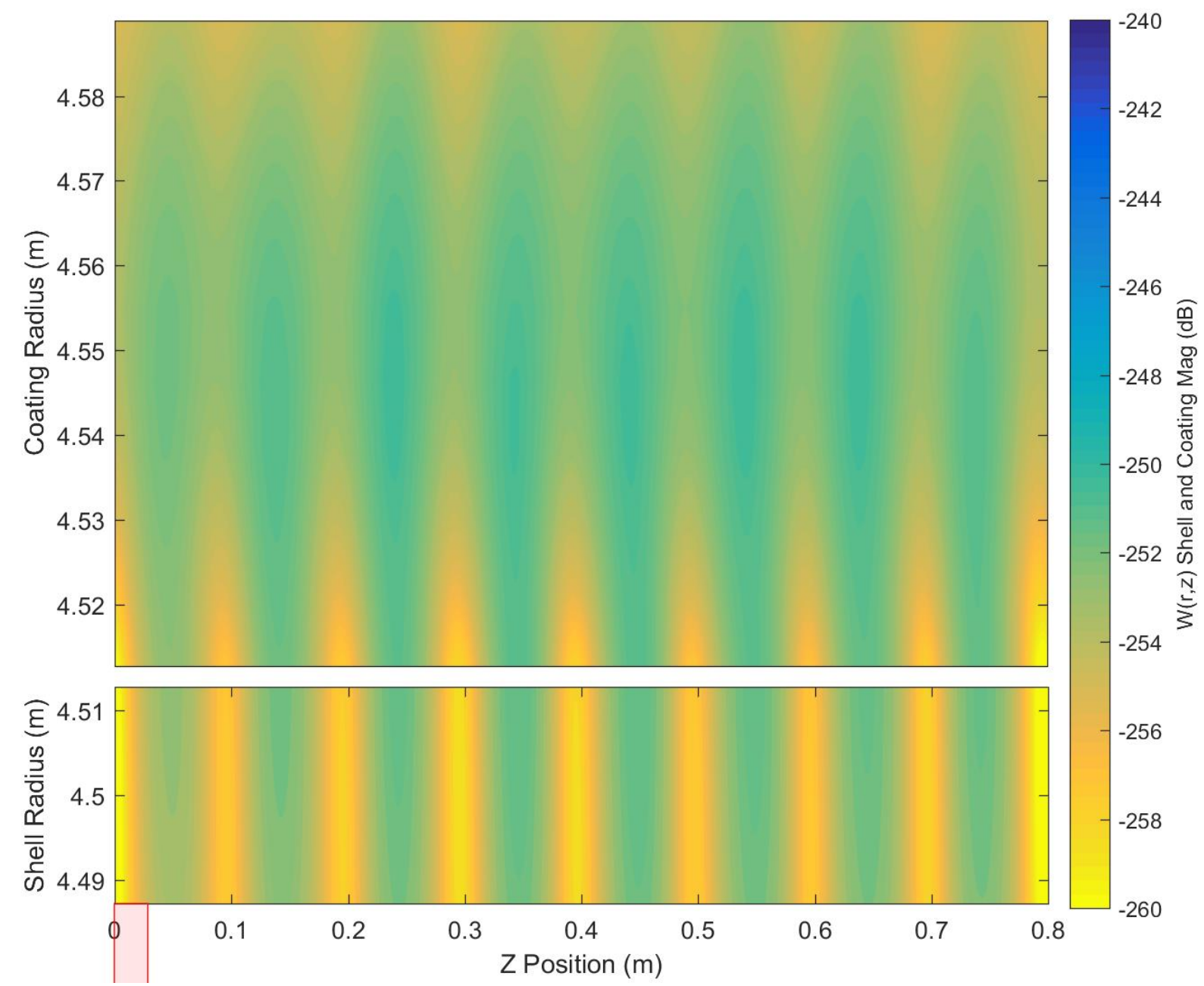

Figure 5.1.10 - Radial displacement field of coated system for cross-section of cylinder thickness at angle $\theta=10 \mathrm{deg}$, for acoustic wave loading $\left(\varphi_{\mathrm{i}}=15 \mathrm{deg}, \mathrm{f}=5000 \mathrm{~Hz}\right)$; red lines represent the rib 


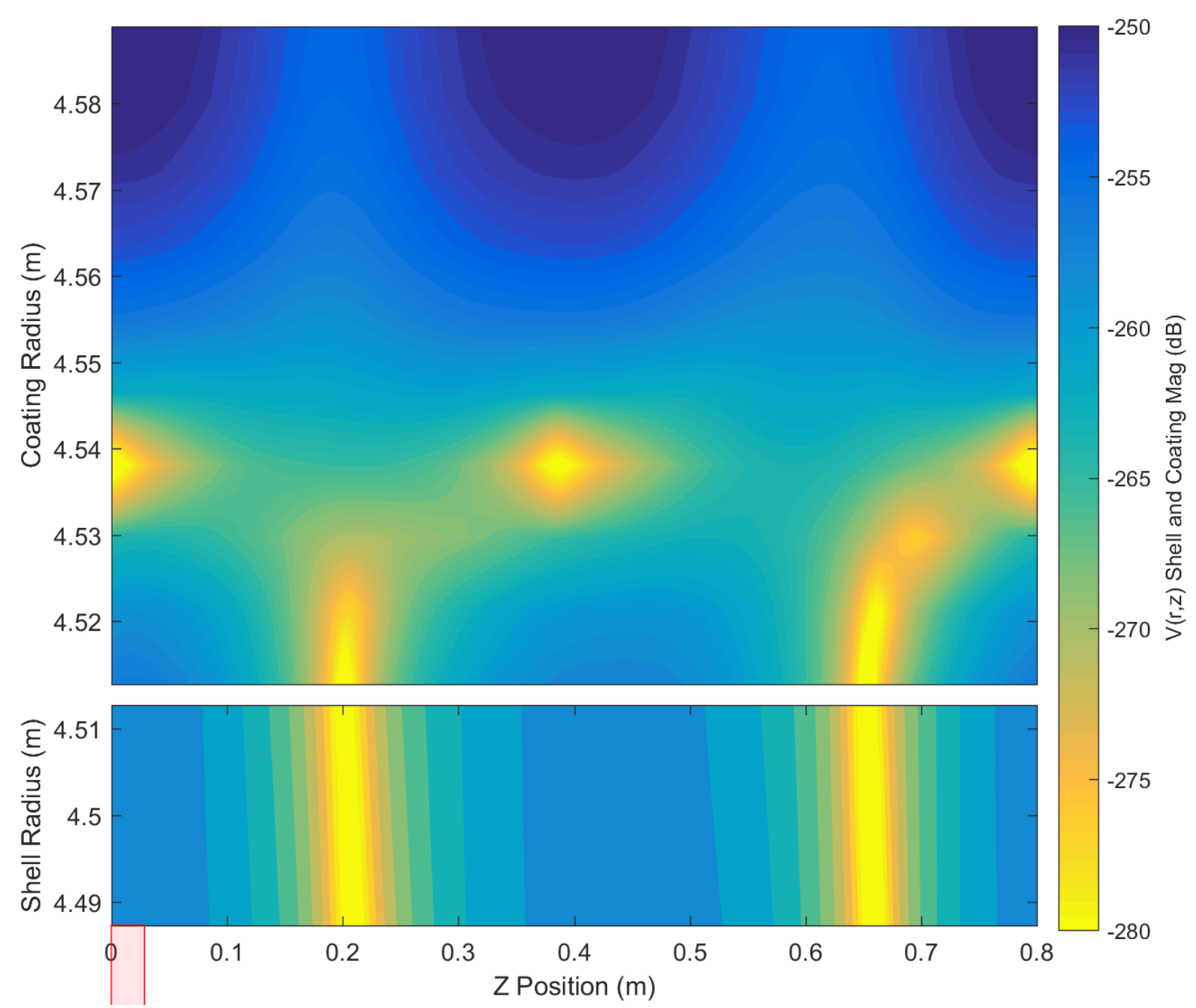

Figure 5.1.11 - Tangential displacement field of coated system for cross-section of cylinder thickness at angle $\theta=10 \mathrm{deg}$, for acoustic wave loading $\left(\varphi_{\mathrm{i}}=15 \mathrm{deg}, \mathrm{f}=5000 \mathrm{~Hz}\right)$; red lines represent the rib 


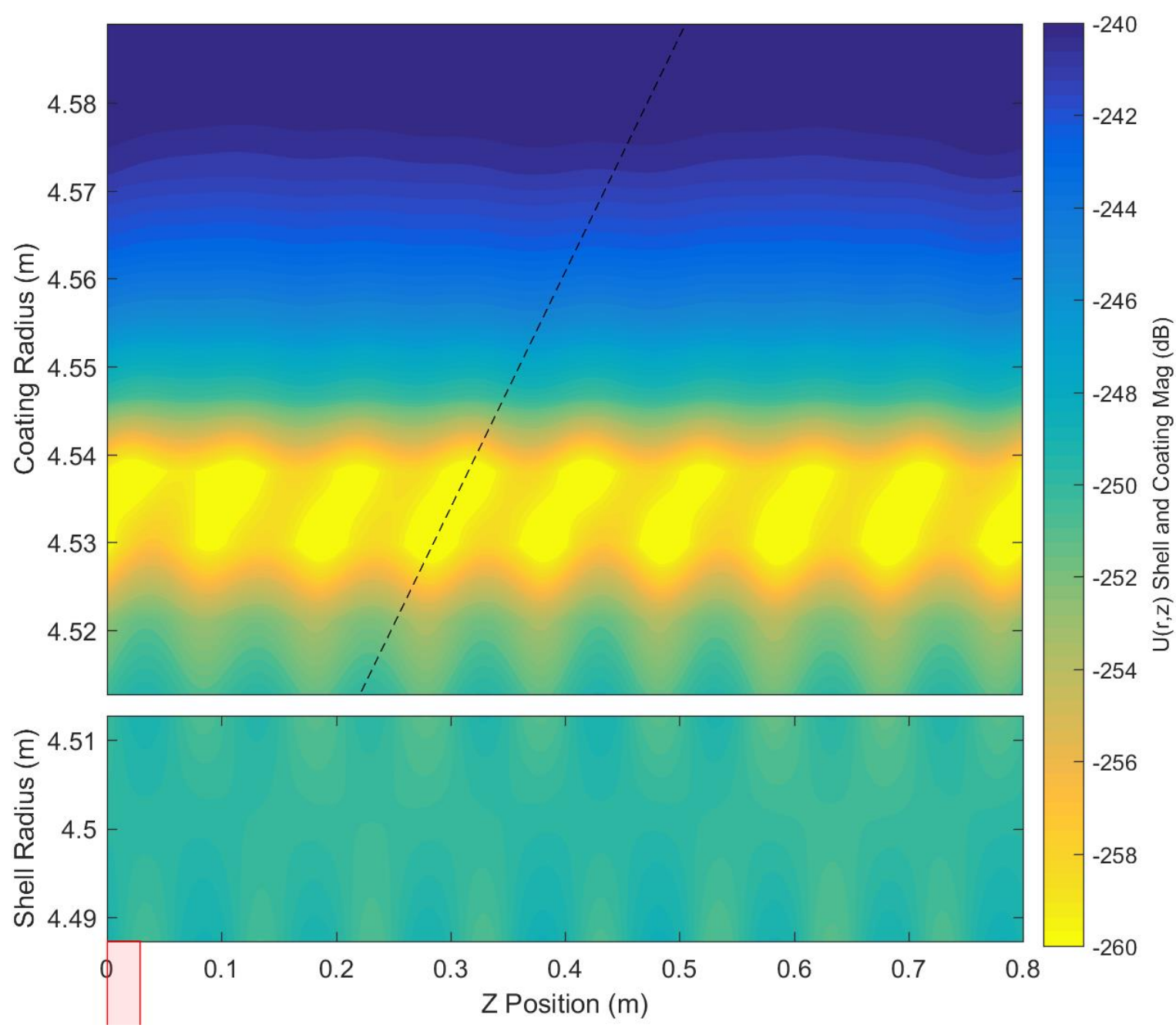

Figure 5.1.12 - Axial displacement field of coated system for cross-section of cylinder thickness at angle $\theta=10 \mathrm{deg}$, for acoustic wave loading $\left(\varphi_{\mathrm{i}}=15 \mathrm{deg}, \mathrm{f}=5000 \mathrm{~Hz}\right)$. Dashed black line represents a reference $15 \mathrm{deg}$ line; red lines represent the rib

The $5000 \mathrm{~Hz}$ acoustic wave input produces a similar radial pattern as the ring load case (Figure 5.1.5), but the axial displacement field features a band of tiny magnitude inside the coating. This band appears to be angled matching the incoming wave incidence angle of $15 \mathrm{deg}$. A black reference line is superimposed on Figure 5.1.12 to indicate the relation. The tangential field shows a low order circumferential shear wave dominating inside the shell that disperses into the coating. These slices are a unique way to explore the spatial response of the shell throughout the thickness, and provide insight how the coating and shell interact.

Radiation pressure ( $\left.P_{\mathrm{rad}}\right)$ is related to the radial motion at the outer surface; while the infinite-impedance pressure $\left(\mathrm{P}_{\text {inf }}\right)$ is independent of the cylinder motion. Together these pressure waves combine to form the scattered pressure, but they can be observed in isolation. Typically, the scattered pressure is measured at a single angular location relative to the object; $\theta=180 \mathrm{deg}$ is commonly referred to as "backscatter". 


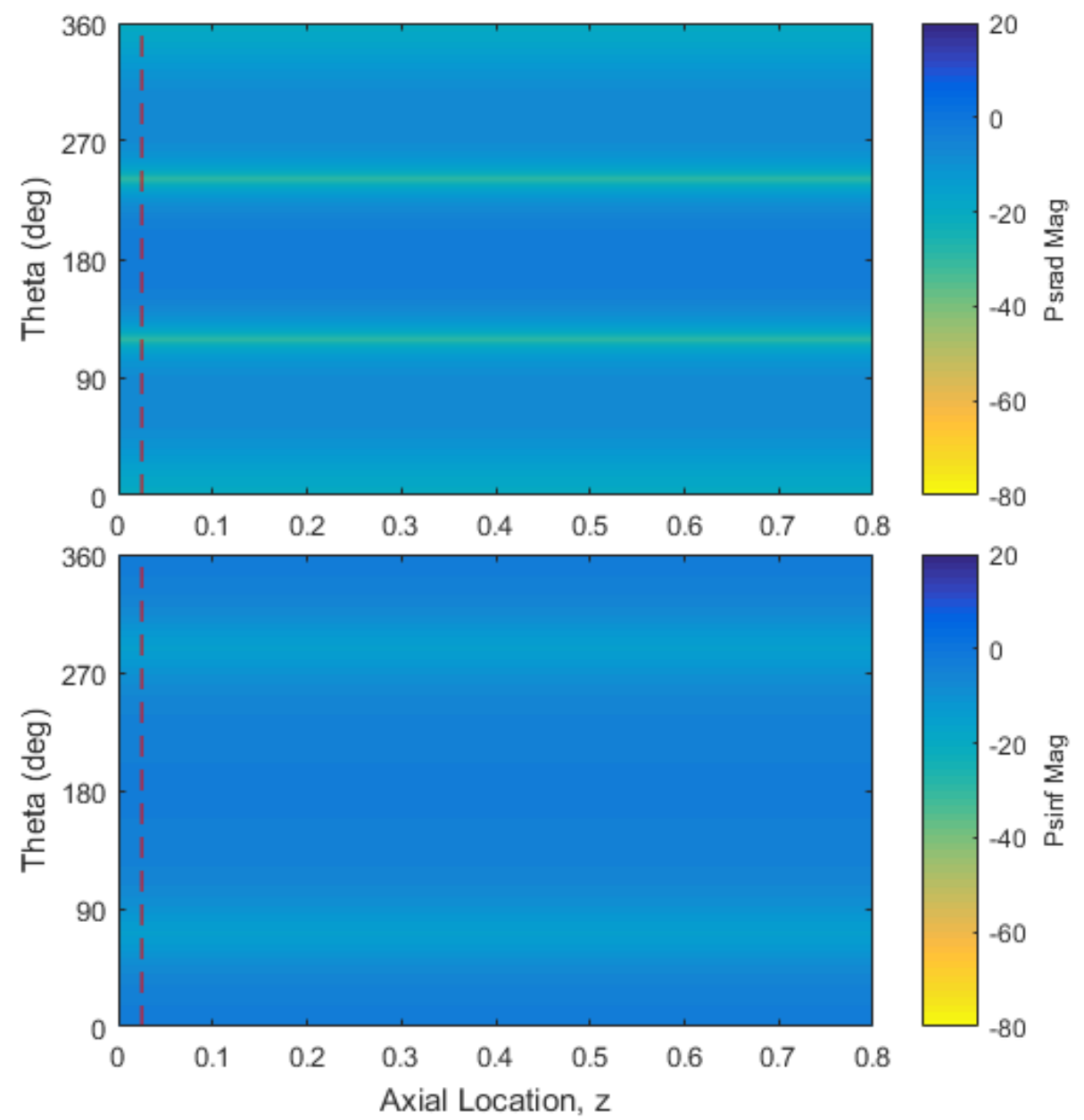

Figure 5.1.10 - Radiated (top), and reflected (bottom) pressure response for acousticwave: $50 \mathrm{~Hz}, \varphi_{\mathrm{i}}=15 \mathrm{deg}$, $\mathrm{mpts}=31$, npts $=7$ 

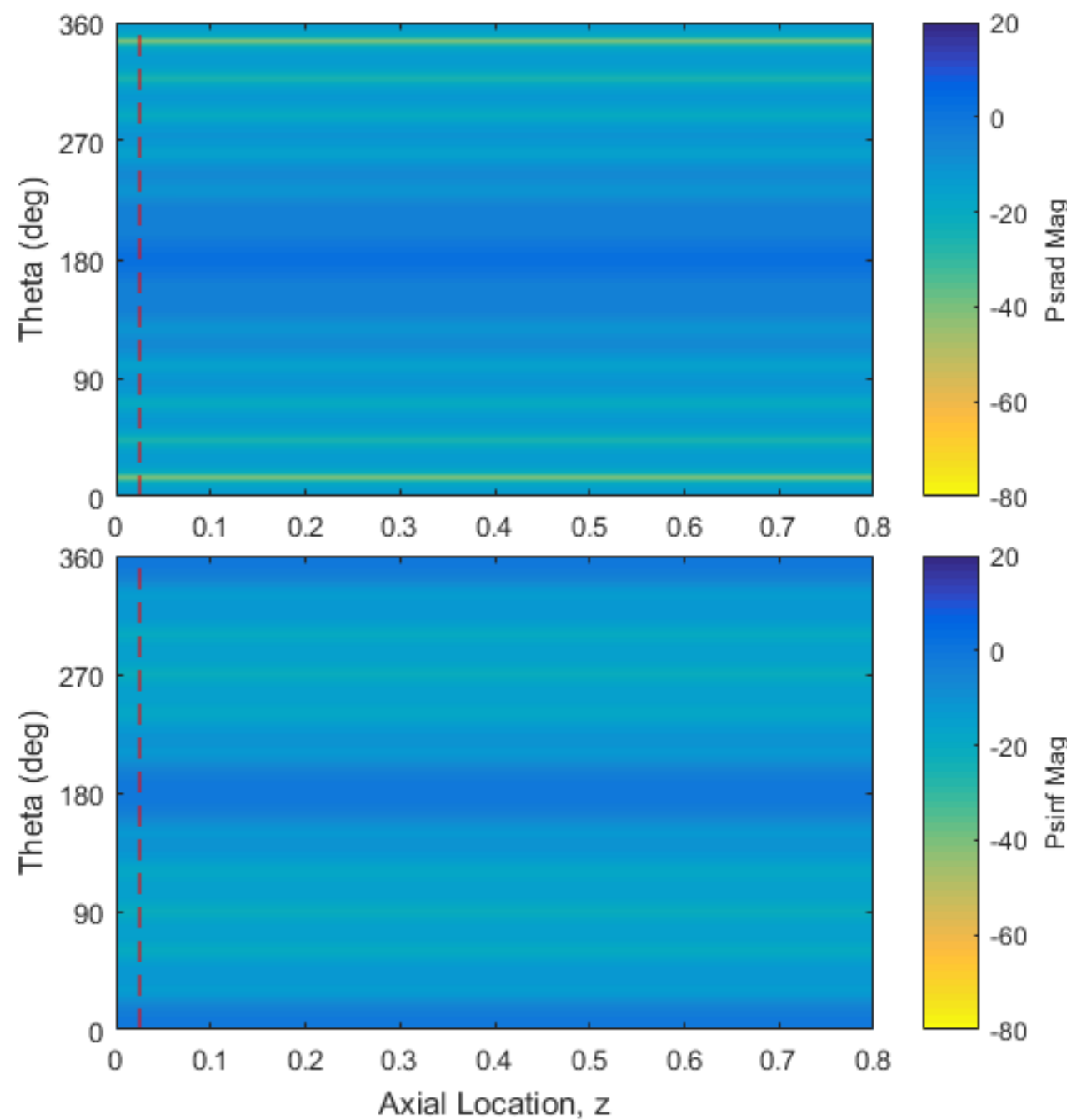

Figure 5.1.11 - Radiated (top), and reflected (bottom) pressure response for acousticwave: $1000 \mathrm{~Hz}, \varphi_{\mathrm{i}}=15 \mathrm{deg}$, mpts $=31$, npts $=7$ 


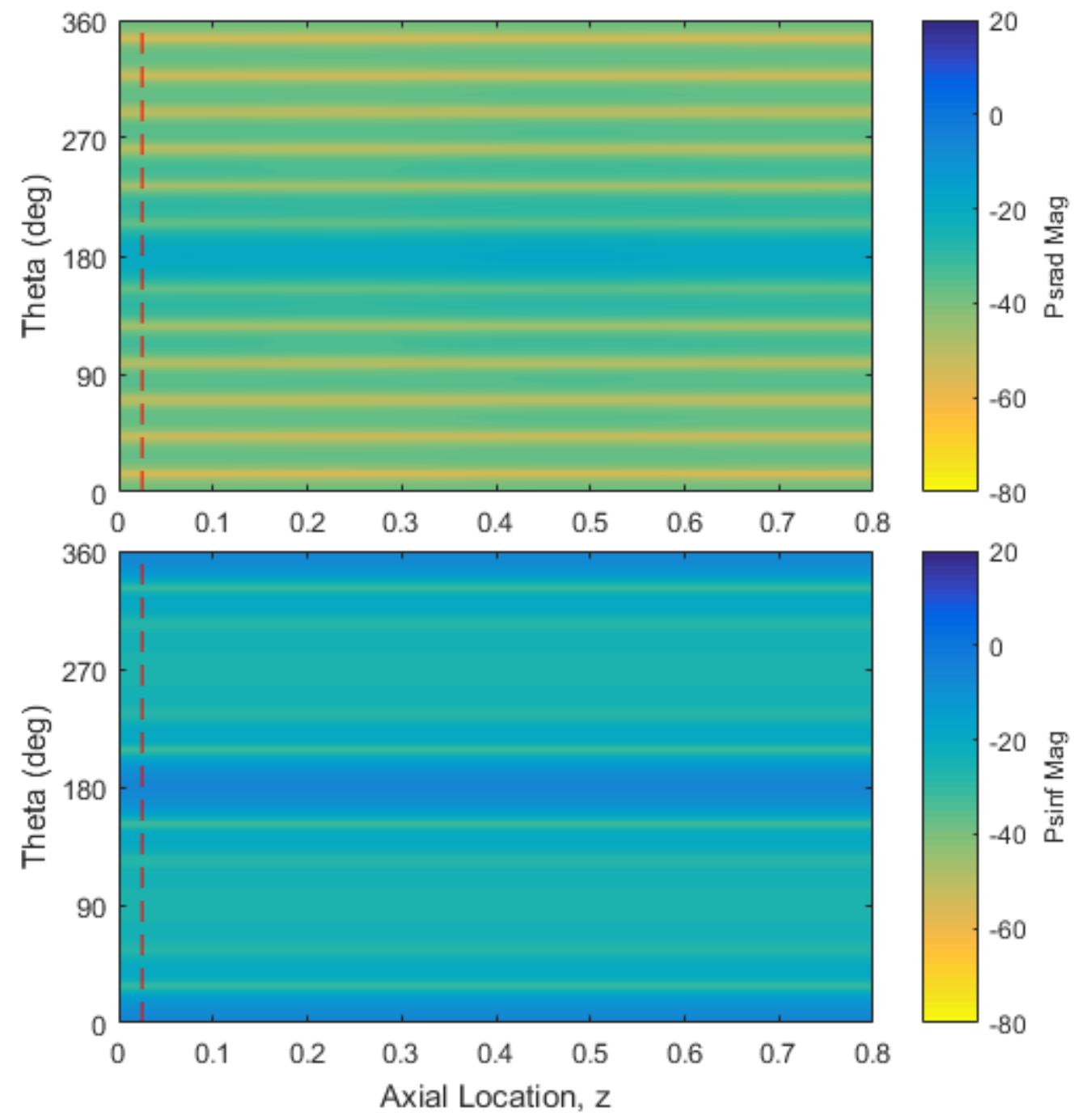

Figure 5.1.12 - Radiated (top), and reflected (bottom) pressure response for acousticwave: $5000 \mathrm{~Hz}, \varphi_{\mathrm{i}}=15 \mathrm{deg}$, mpts $=31$, npts $=7$

Predictably, radiated pressure mirrors the radial displacement response. There is very little axial dependency for radiated pressure and none for reflected pressure at any excitation level. All variation appears to be dependent only on the angular position, $\theta$. Consequently, Figure 5.1.13 and 5.1.15 are more informative. 


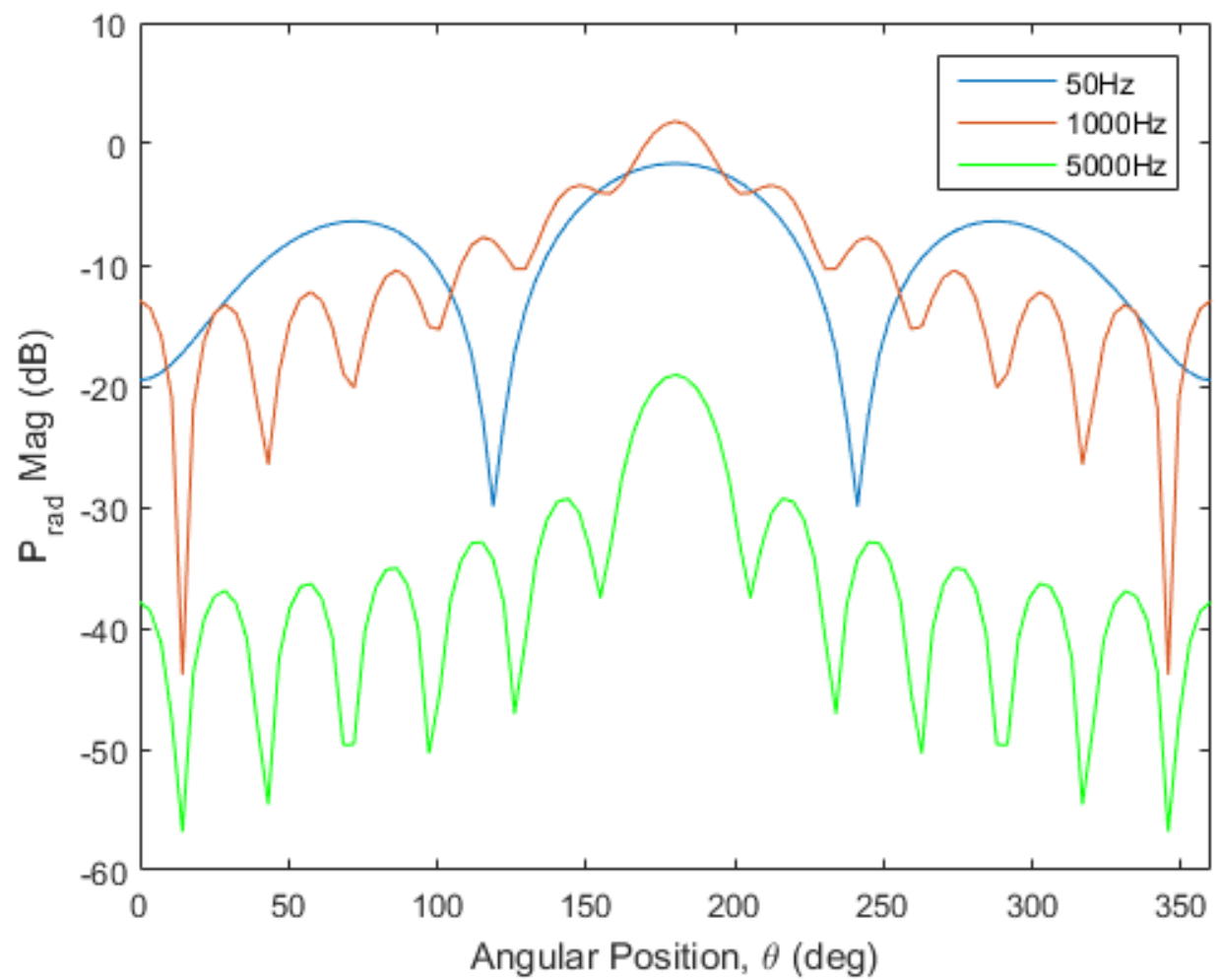

Figure 5.1.13 - Radiated pressure response versus angular position for acoustic-wave: $\varphi_{\mathrm{i}}=$ $15 \mathrm{deg}, \mathrm{mpts}=31, \mathrm{npts}=7$

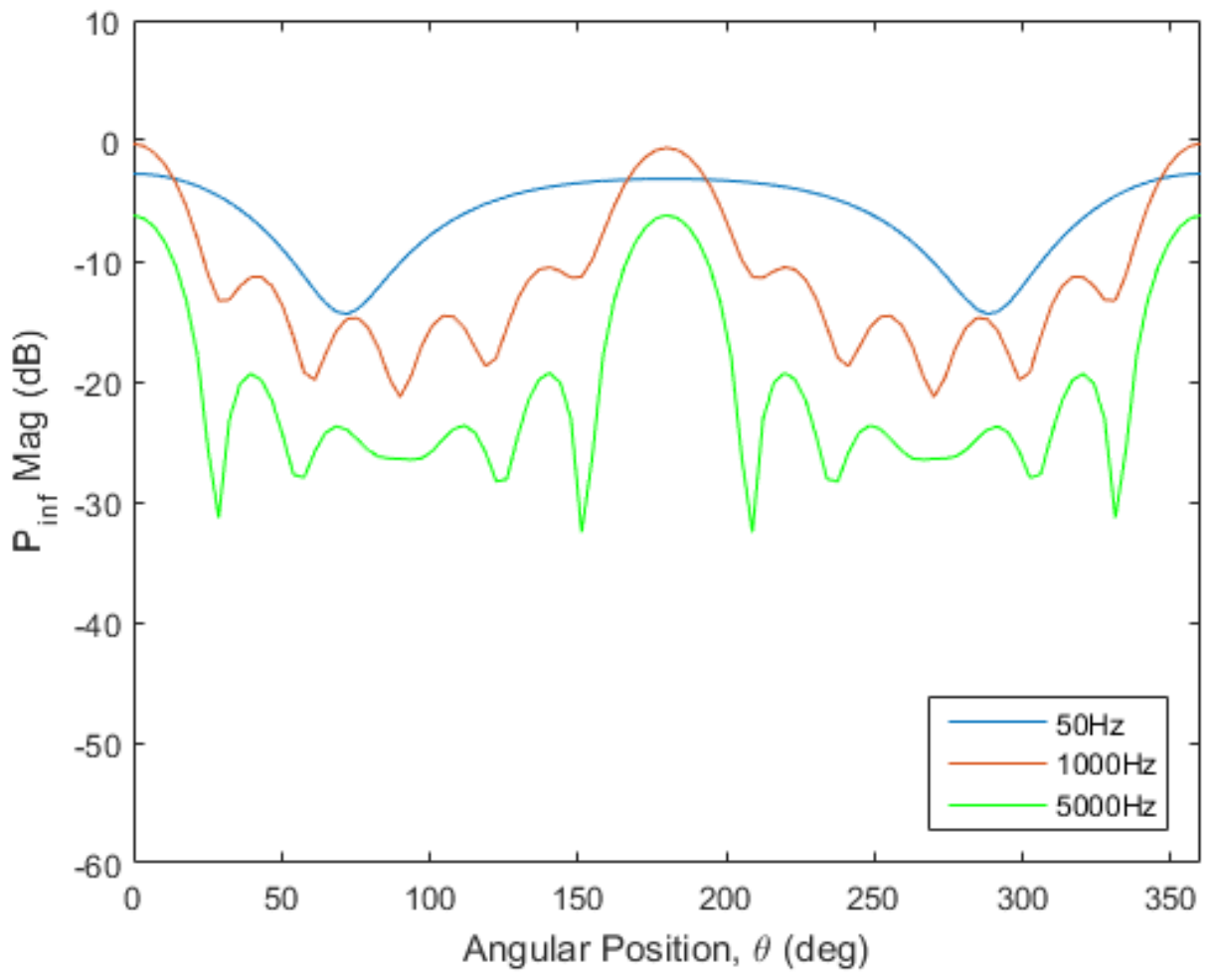

Figure 5.1.14 - Reflected pressure response versus angular position for acoustic-wave: $\varphi_{i}=$ $15 \mathrm{deg}, \mathrm{mpts}=31, \mathrm{npts}=7$ 
The backscatter location is at $180 \mathrm{deg}$ and is a maximum for each excitation. Recall that this measurement is taken 1 meter from the outer surface of the coating. Interestingly, the infiniteimpedance pressure has slightly less magnitude at the backscatter location, but is very similar at $\theta=0$ and $\theta=180 \mathrm{deg}$.

Comparing the radiated and reflected pressures from Model 4 and 5 reveals the effect of the coating on the performance of the system. Figures 5.1.15 and 5.1.16 show the pressure responses at a radial distance of 1 meter from the nominal radius $(5.5 \mathrm{~m})$.
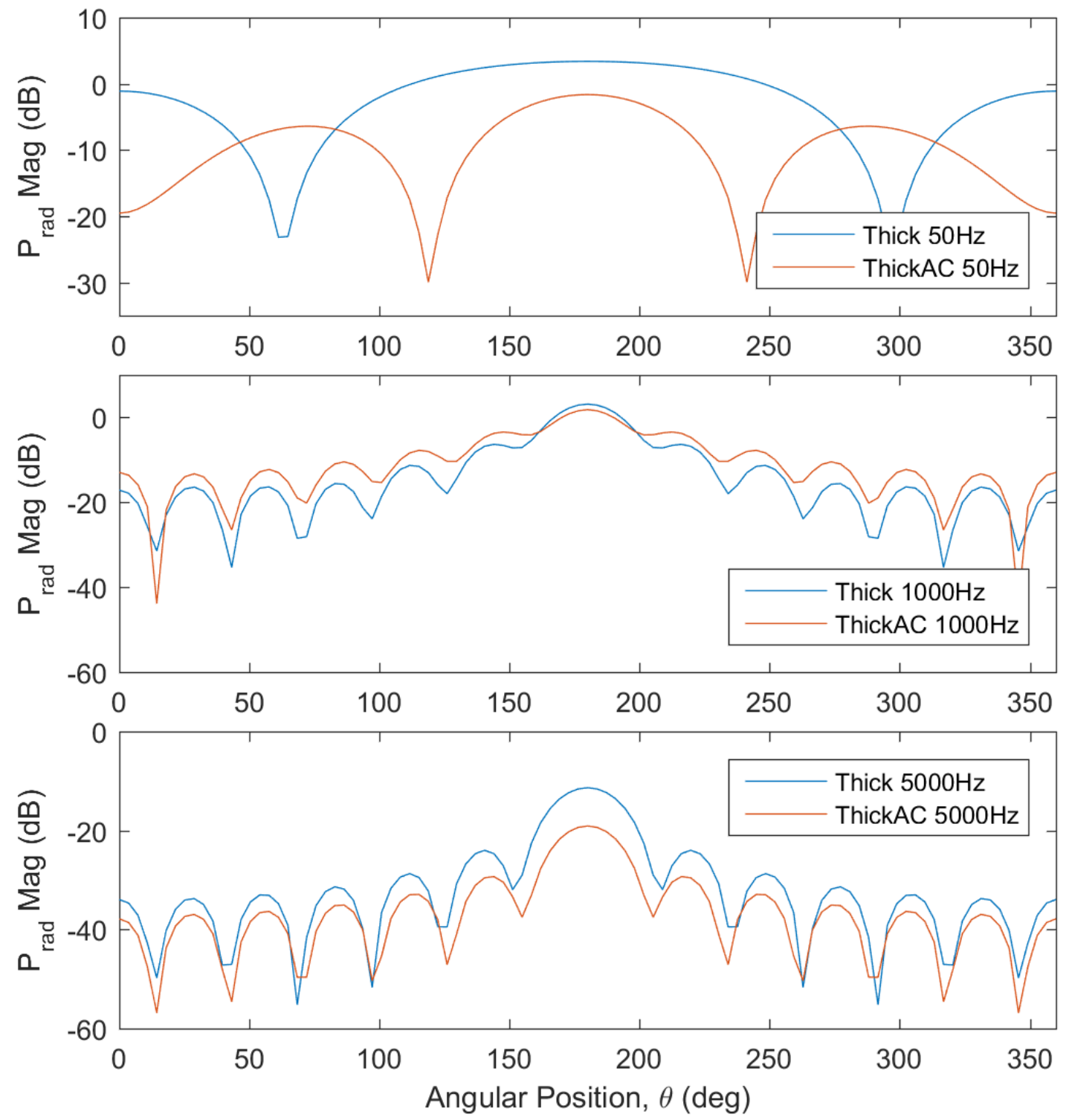

Figure 5.1.15 - Comparison of radiated pressure response between Model 4 and 5 for acoustic-wave: $\varphi_{\mathrm{i}}=15 \mathrm{deg}$, $\mathrm{mpts}=31$, npts $=7$ 

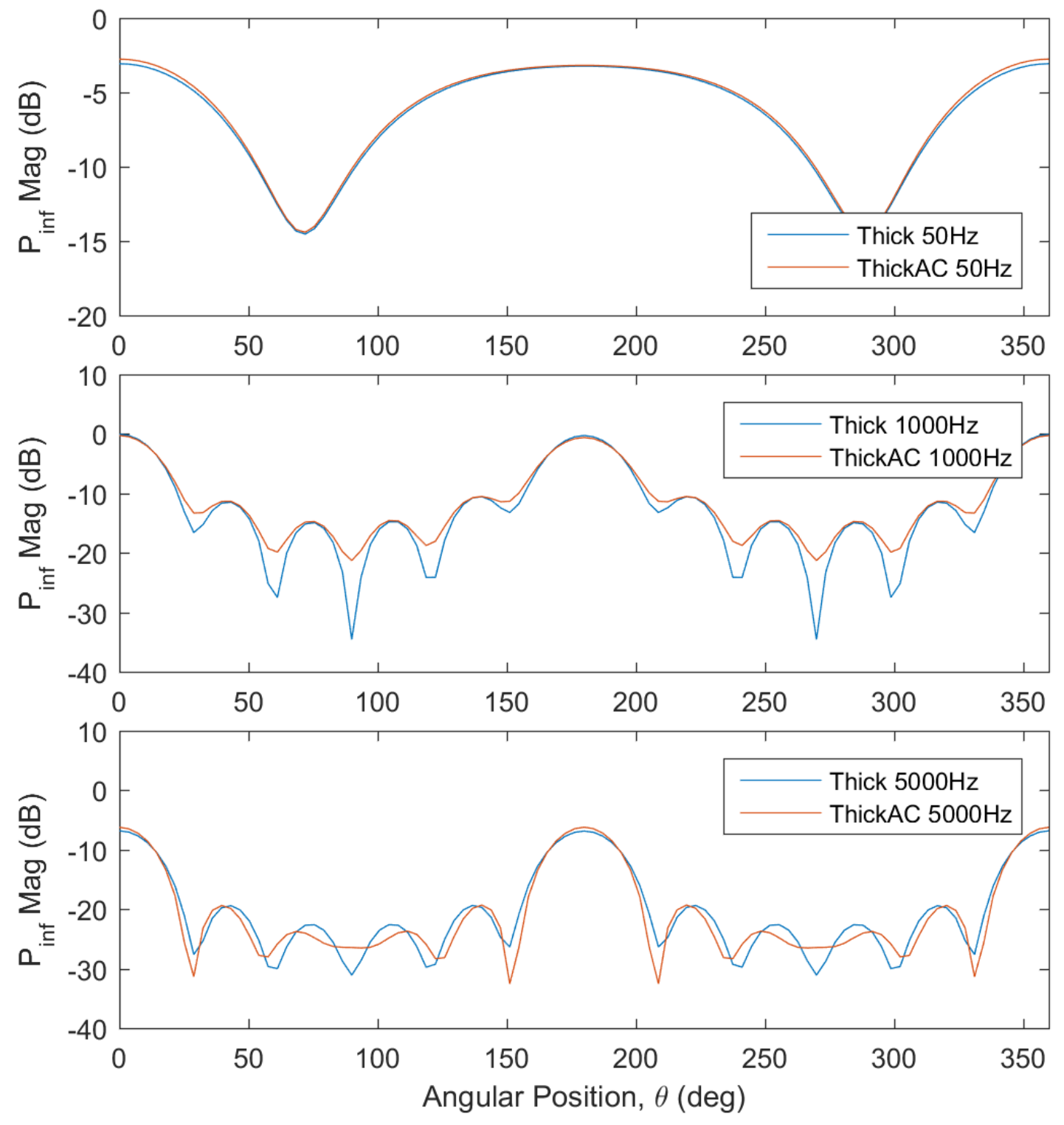

Figure 5.1.16 - Comparison of reflected pressure response between Model 4 and 5 for acoustic-wave: $\varphi_{\mathrm{i}}=15 \mathrm{deg}$, mpts $=31$, npts $=7$

Lower pressure indicate the system is more difficult to detect and is acoustically quieter. This also has an effect on the disturbance of the environment due to vibrations of the structure. Reflected pressure is dependent upon the system geometry, which are equivalent for the two models except for the added surface area from the acoustic coating of Model 5. Therefore, it is not surprising that the reflected pressure is roughly the same. However, there is a substantial difference in radiated pressure. At the $\theta=180 \mathrm{deg}$ angular location, the coated model pressure is $45 \%$ lower at $50 \mathrm{~Hz}, 15 \%$ lower at $1000 \mathrm{~Hz}$, and $55 \%$ lower at $5000 \mathrm{~Hz}$. Obviously, the acoustic coating has a significant effect on the acoustic performance of the shell. 


\section{Chapter 6 Conclusions and Future Work}

The results and performance portrayed in Chapter 5 are discussed along with some possibilities of extending this project in the future.

\section{Conclusions}

An elastic reinforced cylindrical shell model with acoustic coating, finite-length ribs, and fluid loading has been developed. It has been validated using a thin-shell reference system, which certifies that the model behaves appropriately at low frequency and low thickness. The model is then adjusted to match realistic geometry, excited at high frequencies and the displacement and pressure responses are measured.

There are a number of improvements relative to the thin-shell reference model:

1.) Elastic shell requires fewer assumptions compared to the Donnell equation based model. This includes a displacement field dependent upon radial position, such that responses across the thickness are variable and affect the behavior.

2.) Acoustic coating treated as a second elastic body inherits the advantages of elasticity and transmits stress to and from the shell model. Adding an attached body is restricted when using thin-shell approximations.

3.) Fluid loading is incorporated into the shell dynamic equations such that the radiated pressure response is included as a response. The effect of the fluid on the shell is significant.

4.) Beam ribs provide coupling in the radial and tangential directions and includes inertia that is accounted. 
It was shown that the acoustic coating lessened the scattered pressure response by between 15 and $60 \%$, depending on the excitation frequency. Clearly, it has a large effect on making an acoustically quieter system.

\section{Future Work}

While this study has progressed the reinforced shell to a more advanced state, there are still limitations present within the model that could be addressed. In particular, the Timoshenkobeam rib model is certainly superior to linear spring and Euler-Bernoulli based models, in terms of applicable frequency range, but there are more advanced beam models available. Further, it is possible that the elasticity theory applied to the shell system could also be applied to the stiffeners. While parallelization was not a focus for this study, it would certainly help with the long simulation times, when using many modes and excitations.

Validation using a real laboratory experiment would be hugely beneficial and increase confidence in the use of the model developed. This would require a setup with an acoustic pool and non-contact sensors to measure the system displacement. Additional coatings and geometry could be investigated.

Finally, this work can be more effective if it is extended to include finite length structures. This would require a reformulation of the theory and orthogonalization techniques used, but would make a more realistic model. An additional difficulty would be adding end-caps to such a model, where waves would reflect in complex ways. 


\section{References}

1.) Gould, P. Analysis of Plates and Shells, Springer-Verlag, New York, NY (1988).

2.) Graff, K. Wave Motion in Elastic Solids, Dover Publications, New York, NY (1975).

3.) Gazis, D. C. Three-dimensional investigation of the propagation of waves in hollow circular cylinders. I. Analytical foundation, J. Acoust. Soc. Am., 31 (5), 568-573, (1959).

4.) Peloquin, M. S. Forced harmonic vibration of the generally orthotropic cylindrical shell with inner and outer fluid loading, Naval Undersea Warfare Center Technical Report 10,199, New London, Connecticut, (1992).

5.) Smith, P, Sound transmission through thin cylindrical shells, J. Acoust. Soc. Am., 29 (6), 721-729 (1957).

6.) White, R. Elastic wave scattering at a cylindrical discontinuity in a solid, J. Acoust. Soc. Am., 30 (8), 771-785 (1958).

7.) Mead, D. Space-Harmonic analysis of periodically supported beams: Response to convected random loading, Journal of Sound and Vibration, 14 (4), 525-541, (1971).

8.) Mead, D. Free vibration of a thin cylindrical shell with period circumferential stiffeners, Journal of Sound and Vibration, 115 (3), 499-520, (1986).

9.) Bennett, M. S. Free wave propagation in periodically ring stiffened cylindrical shells, Journal of Sound and Vibration, 171 (1), 49-66, (1994).

10.) Beskos, D. E. Dynamic analysis of ring-stiffened circular cylindrical shells, J. Sound Vibration, 75 (1) 1-15 (1981).

11.) Burroughs, C., Acoustic radiation from fluid-loaded infinite circular cylinders with doubly periodic ring supports, J. Acoust. Soc. Am. 75 (3), 715-722 (1984).

12.) Hodges, C. H. The low frequency vibration of a ribbed cylinder, Part 1: Theory, J. Sound Vibration, 101 (2), 219-235, (1985).

13.) Gan, L. Free vibration analysis of ring-stiffened cylindrical shells using wave propagation approach, J. Sound Vibration, 326 (3-5), 633-646, (2009).

14.) Pan, Z. A study on free vibration of a ring-stiffened thin circular cylindrical shell with arbitrary boundary conditions, J. Sound Vibration, 314 (1-2), 330-342, (2008). 
15.) Issa, M. Extensional vibrations of continuous circular curved beams with rotary inertia and shear deformation, I: Free vibration, Journal of Sound and Vibration 114(2), 297-308 (1987).

16.) Liu, Y., A Nonlocal curved beam model based on a modified couple stress theory, International Journal of Structural Stability and Dynamics, 11 (3), 495-512 (2011).

17.) Hull, A. Dynamic response of an elastic plate containing periodic masses, Journal of Sound and Vibration, 310 (1), 1-20 (2008).

18.) Sylvia, J. A dynamic model of a reinforced thin plate with ribs of finite width, Intl. Journal of Acoustics and Vibration, 18 (2), 86-90 (2013).

19.) Hull, A. Response of a cylindrical shell with finite length ring stiffeners, International Journal of Acoustics and Vibration, 21 (3) 317-326 (2016).

20.) Hull, A. Elastic response of an acoustic coating on a rib-stiffened plate. Journal of Sound and Vibration, 329 4192-4211 (2010).

21.) Méresse, P. Acoustic coatings for maritime systems applications using resonant phenomena, C.R. Mecanique, 343, 654-655 (2015).

22.) Cuschieri, J. Influence of circumferential partial coating on the acoustic radiation from a fluid-loaded shell, J. Acoustical Soc. Am., 107 (6), 3196-3207 (2000).

23.) Morse, M. Methods of Theoretical Physics, Part 1, McGraw-Hill Book Company, Inc., New York, p. 297 (1953).

24.) MATLAB Release 2015b, The MathWorks, Inc., Natick, MA, USA.

25.) Advanpix Multiprecision Computing Toolbox, Advanpix LLC, Yokohama, Japan. 


\section{Appendix}

\section{A.1 Proof of Stiffener Index Shift}

In Chapter 2.3, Equation 2.3.16 applied an index shift to eliminate the stiffener exponential and convert Equations 2.3.13-2.3.15 into a more convenient form. The following is performed on 2.3.13, but is representative 2.3.14 and 2.3.15; the final stiffener term of 2.3.13 is written as

$$
\sum_{m=-\infty}^{\infty} \sum_{n=0}^{\infty} U_{m n} \cos (n \theta) e^{i k_{m} z} \sum_{s=-\infty}^{\infty} d_{s} e^{\frac{2 \pi i s z}{L}}
$$

Substituting the indexed axial wavenumber, $\mathrm{k}_{\mathrm{m}}$, according to definition 2.3 .9 and rearranging

$$
e^{i k_{z} z} \sum_{n=0}^{\infty} \sum_{s=-\infty}^{\infty}\left\{\sum_{m=-\infty}^{\infty} U_{m n} e^{\frac{2 \pi i m z}{L}}\right\} d_{s} e^{\frac{2 \pi i s z}{L}} \cos (n \theta)
$$

Expanding the $m=s u m m a t i o n$ yields

$$
e^{i k_{z} Z} \sum_{n=0}^{\infty} \sum_{s=-\infty}^{\infty}\left\{\ldots+U_{-1 n} e^{\frac{2 \pi i(-1) z}{L}}+U_{0 n} e^{\frac{2 \pi i(0) z}{L}}+U_{1 n} e^{\frac{2 \pi i(1) z}{L}}+\cdots\right\} d_{s} e^{\frac{2 \pi i s z}{L}} \cos (n \theta)
$$

And the expanding the s-summation results in

$$
\begin{aligned}
e^{i k_{z} z} \sum_{n=0}^{\infty}[\cdots+ & \left\{\ldots+U_{-1 n} e^{\frac{2 \pi i(-1) z}{L}}+U_{0 n} e^{\frac{2 \pi i(0) z}{L}}+U_{1 n} e^{\frac{2 \pi i(1) z}{L}}+\cdots\right\} d_{-1} e^{\frac{2 \pi i(-1) z}{L}} \\
& +\left\{\ldots+U_{-1 n} e^{\frac{2 \pi i(-1) z}{L}}+U_{0 n} e^{\frac{2 \pi i(0) z}{L}}+U_{1 n} e^{\frac{2 \pi i(1) z}{L}}+\cdots\right\} d_{0} e^{\frac{2 \pi i(0) z}{L}} \\
& +\left\{\ldots+U_{-1 n} e^{\frac{2 \pi i(-1) z}{L}}+U_{0 n} e^{\frac{2 \pi i(0) z}{L}}+U_{1 n} e^{\frac{2 \pi i(1) z}{L}}+\cdots\right\} d_{1} e^{\frac{2 \pi i(1) z}{L}} \\
& +\cdots] \cos (n \theta)
\end{aligned}
$$

Multiplying the outer exponentials with the inners gives

$$
\begin{aligned}
e^{i k_{z} z} \sum_{n=0}^{\infty}[\cdots+ & \left\{\cdots+U_{-1 n} d_{-1} e^{\frac{2 \pi i(-2) z}{L}}+U_{0 n} d_{-1} e^{\frac{2 \pi i(-1) z}{L}}+U_{1 n} d_{-1} e^{\frac{2 \pi i(0) z}{L}}+\cdots\right\} \\
& +\left\{\cdots+U_{-1 n} d_{0} e^{\frac{2 \pi i(-1) z}{L}}+U_{0 n} d_{0} e^{\frac{2 \pi i(0) z}{L}}+U_{1 n} d_{0} e^{\frac{2 \pi i(1) z}{L}}+\cdots\right\} \\
& +\left\{\cdots+U_{-1 n} d_{1} e^{\frac{2 \pi i(0) z}{L}}+U_{0 n} d_{1} e^{\frac{2 \pi i(1) z}{L}}+U_{1 n} d_{1} e^{\frac{2 \pi i(2) z}{L}}+\cdots\right\} \\
& +\cdots] \cos (n \theta)
\end{aligned}
$$


Now the square bracketed term can be individually summed using common exponentials

$$
\begin{aligned}
e^{i k_{z} z} \sum_{n=0}^{\infty}[ & \cdots+\sum_{s=-\infty}^{\infty} U_{s n} d_{-s} e^{\frac{2 \pi i(-1) z}{L}}+\sum_{s=-\infty}^{\infty} U_{s n} d_{-s} e^{\frac{2 \pi i(0) z}{L}} \\
& \left.+\sum_{s=-\infty}^{\infty} U_{s n} d_{-s} e^{\frac{2 \pi i(1) z}{L}}+\cdots\right] \cos (n \theta)
\end{aligned}
$$

The summations within the square brackets can be incorporated into an m-summation

$$
e^{i k_{z} z} \sum_{n=0}^{\infty}\left[\sum_{s=-\infty}^{\infty} \sum_{m=-\infty}^{\infty} U_{s n} d_{m-s} e^{\frac{2 \pi i m z}{L}}\right] \cos (n \theta)
$$

Finally, the constant exponential in front can be reintroduced into the summations

$$
\sum_{n=0}^{\infty}\left[\sum_{s=-\infty}^{\infty} \sum_{m=-\infty}^{\infty} U_{s n} d_{m-s} e^{i k_{m} z}\right] \cos (n \theta)
$$

which results in the final form given in Equation 2.3.16

$$
\sum_{m=-\infty}^{\infty} \sum_{n=0}^{\infty} U_{m n} \cos (n \theta) e^{i k_{m} z} \sum_{s=-\infty}^{\infty} d_{s} e^{\frac{2 \pi i s z}{L}}=\sum_{n=0}^{\infty}\left[\sum_{s=-\infty}^{\infty} \sum_{m=-\infty}^{\infty} U_{s n} d_{m-s} e^{i k_{m} z}\right] \cos (n \theta)
$$

\section{A.2 Elements of Thin-Shell A Matrix}

The $A_{m n}$ matrix introduced in Chapter 2.3 Equation 2.3.28 is defined as a $3 \times 3$ matrix with the following elements, shown in [row, column] format:

$$
\begin{gathered}
A_{m n}[1,1]=h c_{p}^{2} k_{m}^{2}+\frac{\rho h c_{p}^{2}(1-v) n^{2}}{2 a^{2}}-\rho h \omega^{2} \\
A_{m n}[1,2]=-\frac{\rho h c_{p}^{2}(1+v) i k_{m} n}{2 a} \\
A_{m n}[1,3]=-\frac{\rho h c_{p}^{2} v i k_{m}}{a} \\
A_{m n}[2,1]=\frac{\rho h c_{p}^{2}(1+v) i k_{m} n}{2 a} \\
A_{m n}[2,2]=\frac{\rho h c_{p}^{2}(1-v) k_{m}^{2}}{2}+\frac{\rho h c_{p}^{2} n^{2}}{a^{2}}-\rho h \omega^{2}
\end{gathered}
$$




$$
\begin{gathered}
A_{m n}[2,3]=\frac{\rho h c_{p}^{2} n}{a^{2}} \\
A_{m n}[3,1]=\frac{\rho h c_{p}^{2} v i k_{m}}{a} \\
A_{m n}[3,2]=\frac{\rho h c_{p}^{2} n}{a^{2}} \\
A_{m n}[3,3]=\frac{\rho h c_{p}^{2}}{a^{2}}+\frac{\rho h^{3} c_{p}^{2} k_{m}^{4}}{12}+\frac{\rho h^{3} c_{p}^{2} k_{m}^{2} n^{2}}{6 a^{2}}+\frac{\rho h c_{p}^{2} n^{4}}{12 a^{4}}-\rho h \omega^{2}
\end{gathered}
$$

\section{A.3 Elements of Thick-Shell A Matrix}

The $A_{m n}$ matrix introduced in Chapter 2.4 Equation 2.4.39 is defined as a $6 \times 6$ matrix with the following elements, shown in [row, column] format:

$$
\begin{aligned}
& A_{m n}[1,1]=\frac{2 \alpha_{m} \mu}{a} J_{n+1}\left(\alpha_{m} a\right)+\left(\frac{2 n(n-1) \mu}{a^{2}}-\alpha_{m}^{2} \lambda-2 \alpha_{m}^{2} \mu-k_{z m}^{2} \lambda\right) J_{n}\left(\alpha_{m} a\right) \\
& A_{m n}[1,2]=\frac{2 \alpha_{m} \mu}{a} Y_{n+1}\left(\alpha_{m} a\right)+\left(\frac{2 n(n-1) \mu}{a^{2}}-\alpha_{m}^{2} \lambda-2 \alpha_{m}^{2} \mu-k_{z m}^{2} \lambda\right) Y_{n}\left(\alpha_{m} a\right) \\
& A_{m n}[1,3]=-\frac{2 n \beta_{m} \mu}{a} J_{n+1}\left(\beta_{m} a\right)+\frac{2 n(n-1) \mu}{a^{2}} J_{n}\left(\beta_{m} a\right) \\
& A_{m n}[1,4]=-\frac{2 n \beta_{m} \mu}{a} Y_{n+1}\left(\beta_{m} a\right)+\frac{2 n(n-1) \mu}{a^{2}} Y_{n}\left(\beta_{m} a\right) \\
& A_{m n}[1,5]=-\frac{2 n(n+1) i k_{m} \mu}{a} J_{n+1}\left(\beta_{m} a\right)+2 i k_{m} \beta_{m} \mu J_{n}\left(\beta_{m} a\right) \\
& A_{m n}[1,6]=-\frac{2 n(n+1) i k_{m} \mu}{a} Y_{n+1}\left(\beta_{m} a\right)+2 i k_{m} \beta_{m} \mu Y_{n}\left(\beta_{m} a\right) \\
& A_{m n}[2,1]=\frac{2 n \alpha_{m} \mu}{a} J_{n+1}\left(\alpha_{m} a\right)-\frac{2 n(n-1) \mu}{a^{2}} J_{n}\left(\alpha_{m} a\right) \\
& A_{m n}[2,2]=\frac{2 n \alpha_{m} \mu}{a} Y_{n+1}\left(\alpha_{m} a\right)-\frac{2 n(n-1) \mu}{a^{2}} Y_{n}\left(\alpha_{m} a\right) \\
& A_{m n}[2,3]=-\frac{\beta_{m} \mu}{a} J_{n+1}\left(\beta_{m} a\right)+\left(\beta_{m}^{2} \mu-\frac{2 n(n-1) \mu}{a^{2}}\right) J_{n}\left(\beta_{m} a\right)
\end{aligned}
$$




$$
\begin{gathered}
A_{m n}[2,4]=-\frac{\beta_{m} \mu}{a} Y_{n+1}\left(\beta_{m} a\right)+\left(\beta_{m}^{2} \mu-\frac{2 n(n-1) \mu}{a^{2}}\right) Y_{n}\left(\beta_{m} a\right) \\
A_{m n}[2,5]=-\frac{2(n+1) i k_{m} \mu}{a} J_{n+1}\left(\beta_{m} a\right)+i k_{m} \beta_{m} \mu J_{n}\left(\beta_{m} a\right) \\
A_{m n}[2,6]=-\frac{2(n+1) i k_{m} \mu}{a} Y_{n+1}\left(\beta_{m} a\right)+i k_{m} \beta_{m} \mu Y_{n}\left(\beta_{m} a\right) \\
A_{m n}[3,1]=-2 i k_{m} \alpha_{m} J_{n+1}\left(\alpha_{m} a\right)+\frac{2 n i k_{m} \mu}{a} J_{n}\left(\alpha_{m} a\right) \\
A_{m n}[3,2]=-2 i k_{m} \alpha_{m} Y_{n+1}\left(\alpha_{m} a\right)+\frac{2 n i k_{m} \mu}{a} Y_{n}\left(\alpha_{m} a\right) \\
A_{m n}[3,3]=\frac{n i k_{m} \mu}{a} J_{n}\left(\beta_{m} a\right) \\
A_{m n}[3,4]=\frac{n i k_{m} \mu}{a} Y_{n}\left(\beta_{m} a\right) \\
A_{m n}[3,5]=\mu\left(\beta_{m}^{2}-k_{m}^{2}\right) J_{n+1}\left(\beta_{m} a\right)-\frac{n \beta_{m} \mu}{a} J_{n}\left(\beta_{m} a\right) \\
\mu\left(\beta_{m}^{2}-k_{m}^{2}\right) Y_{n+1}\left(\beta_{m} a\right)-\frac{n \beta_{m} \mu}{a} Y_{n}\left(\beta_{m} a\right)
\end{gathered}
$$

Rows $4-6$ are identical to rows $1-3$ except that radius $a$ is replaced by radius $c$.

\section{A.4 Timoshenko Curved Beam Equations}

Following the work of Issa [15], deriving the equations of motion for the ring stiffeners using Timoshenko beams, involves a free-body-diagram (FBD) of an infinitesimal section of the $r, \theta$ plane, shown below in Figure A.3.1 


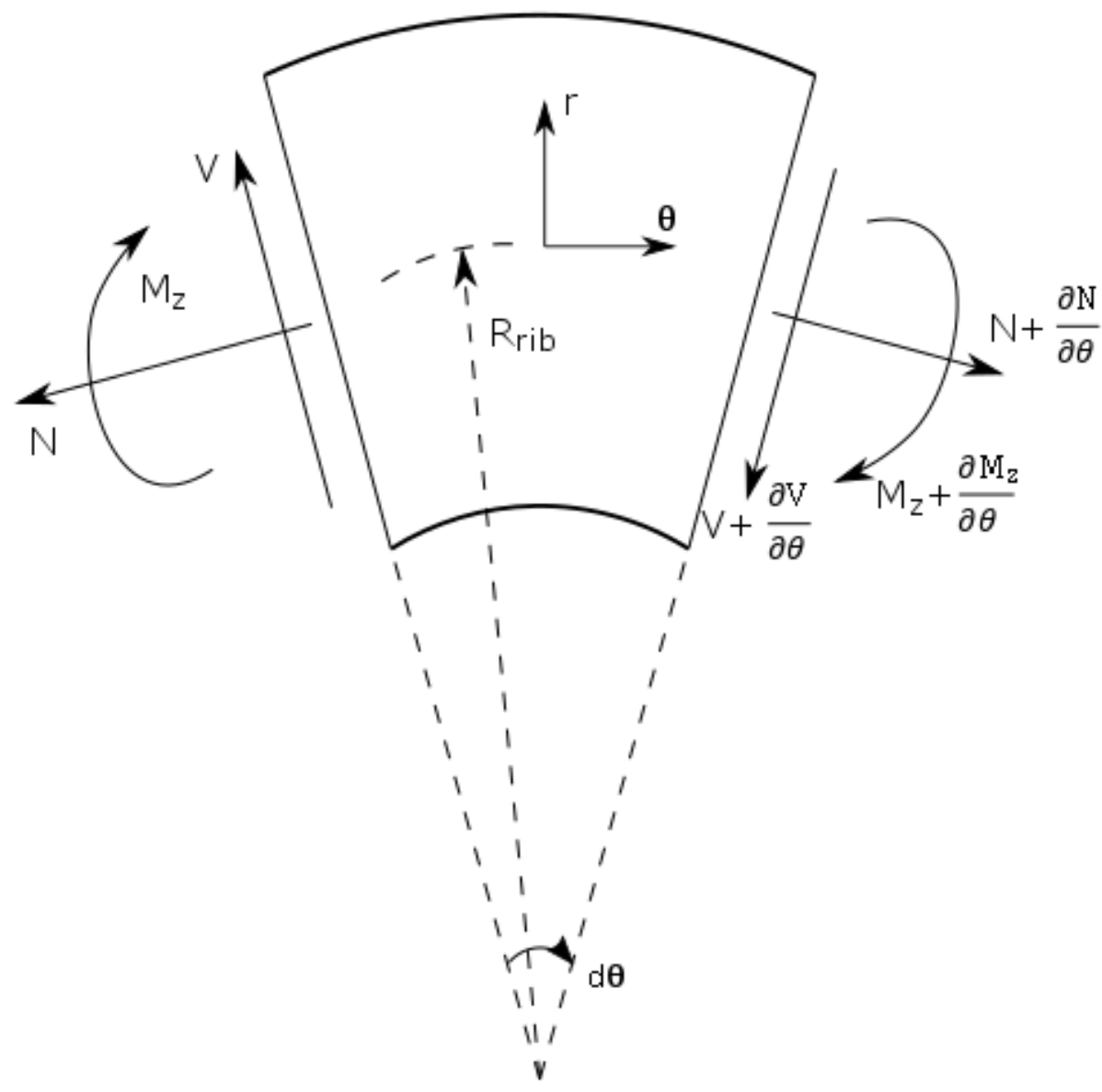

Figure A.3.1 - FBD of infinitesimal section of circular beam

where $\mathrm{V}$ and $\mathrm{N}$ are the shear and tangential forces, respectively, $\mathrm{M}_{\mathrm{z}}$ is the moment about the $\mathrm{z}$ axis (out-of-plane). $R$ is the ring radius of curvature of the neutral axis. Summing forces in the $r$ and $\theta$ directions, and moments in the $z$ direction results in the following equilibrium equations

$$
\begin{aligned}
& -V+\left(V+\frac{\partial V}{\partial \theta} d \theta\right)-\left(N+\frac{\partial N}{\partial \theta} d \theta\right) d \theta=\rho A R \frac{\partial^{2} w}{\partial t^{2}} d \theta \\
& -N+\left(N+\frac{\partial N}{\partial \theta} d \theta\right)-\left(V+\frac{\partial V}{\partial \theta} d \theta\right) d \theta=\rho A R \frac{\partial^{2} v}{\partial t^{2}} d \theta \\
& M-\left(M+\frac{\partial M}{\partial \theta} d \theta\right)-\left(V+\frac{\partial V}{\partial \theta} d \theta\right) R d \theta=\rho I R \frac{\partial^{2} \psi}{\partial t^{2}} d \theta
\end{aligned}
$$

in which $I$ is the moment of inertia, $w$ is the radial rib displacement, $v$ is the circumferential rib displacement and $\psi$ is the angular deflection due to bending. First terms of order $(d \theta)^{2}$ are approximately 0 , and then the remaining $d \theta$ term can be canceled in each equation leaving 


$$
\begin{aligned}
& \frac{\partial V}{\partial \theta}-N=\rho A R \frac{\partial^{2} w}{\partial t^{2}} \\
& \frac{\partial N}{\partial \theta}+V=\rho A R \frac{\partial^{2} v}{\partial t^{2}} \\
& \frac{\partial M}{\partial \theta}+R V=\rho I R \frac{\partial^{2} \psi}{\partial t^{2}}
\end{aligned}
$$

The total angular deformation of the neutral axis compared to the undeformed tangential axis consists of a rotation due to bending $(\psi)$ and shear $(\beta)$

$$
\varphi=\psi+\beta
$$

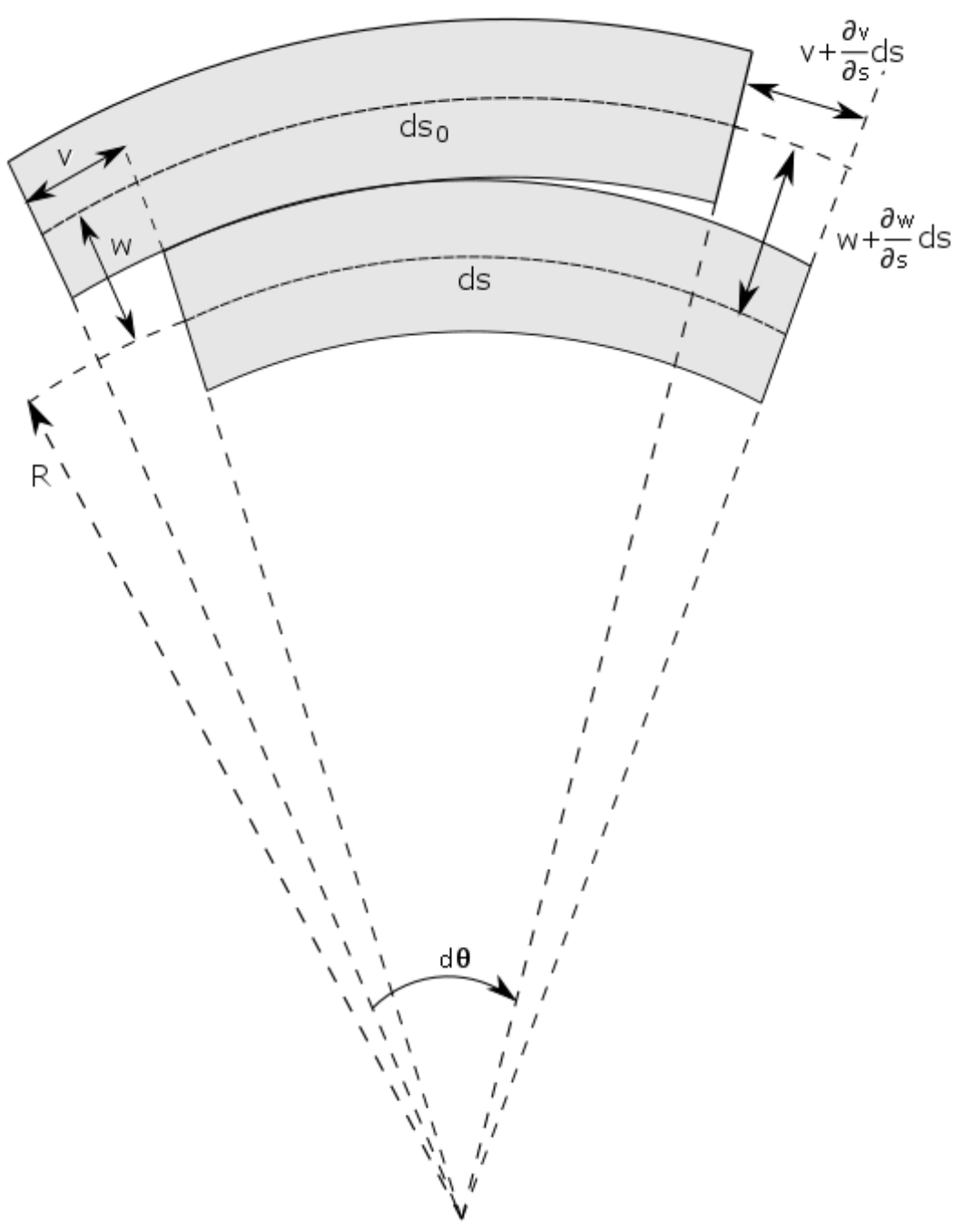

Figure A.3.2 - Curved beam element undergoing deformation and rotation

Figure [*] shows the deformation and rotation of a curved beam element, compared to its initial state $(\mathrm{ds})$. Rotation of the element due to tangential and radial displacement is calculated as 


$$
\varphi_{v}=\frac{v}{R}, \quad \varphi_{w}=\frac{u+\frac{\partial w}{\partial s} d s-u}{d s}=\frac{\partial w}{\partial s}=\frac{1}{R} \frac{\partial w}{\partial \theta}, \quad \varphi=\frac{1}{R}\left(v+\frac{\partial w}{\partial \theta}\right)
$$

Thus the angle due to shear can be determined as

$$
\beta=\frac{1}{R}\left(v+\frac{\partial w}{\partial \theta}-R \psi\right)
$$

while the extensional deformation of the curved beam is the change in length, ds, given as

$$
d e=d s-d s_{0}=\left[(R-w) d \theta+\left(v+\frac{\partial v}{\partial s} d s\right)-v\right]-R d \theta=\left(\frac{\partial v}{\partial \theta}-w\right) d \theta
$$

From the rotation and extension of the infinitesimal beam segment, the normal and shear forces and moment can be found using elementary beam theory

$$
\begin{gathered}
N=E A \frac{d e}{d s}=\frac{E A}{R} \frac{d e}{d \theta}=\frac{E A}{R}\left(\frac{\partial v}{\partial \theta}-w\right) \\
V=K_{s} G A \beta=\frac{K_{s} G A}{R}\left(v+\frac{\partial w}{\partial \theta}-R \psi\right) \\
M=-\frac{E I}{R} \frac{\partial \psi}{\partial \theta}
\end{gathered}
$$

where $\mathrm{E}$ is Young's modulus, $\mathrm{G}$ is the shear modulus, and $\mathrm{K}_{\mathrm{s}}$ is the shape factor. Substituting these into the equilibrium equations results in the final Timoshenko curved beam equations of motion in the radial direction

$$
\frac{E A}{R}\left(\frac{\partial v}{\partial \theta}-w\right)+\frac{K_{S} G A}{R}\left(\frac{\partial^{2} w}{\partial \theta^{2}}+\frac{\partial v}{\partial \theta}-R \frac{\partial \psi}{\partial \theta}\right)+f_{r}=\rho A R \frac{\partial^{2} w}{\partial t^{2}}
$$

in the circumferential direction

$$
\frac{E A}{R}\left(\frac{\partial^{2} v}{\partial \theta^{2}}-\frac{\partial w}{\partial \theta}\right)-\frac{K_{S} G A}{R}\left(\frac{\partial w}{\partial \theta}+v-R \psi\right)+f_{\theta}=\rho A R \frac{\partial^{2} v}{\partial t^{2}}
$$

and in the z-rotational direction

$$
\frac{E I}{R} \frac{\partial^{2} \psi}{\partial \theta^{2}}+K_{s} G A\left(\frac{\partial v}{\partial \theta}+w-R \psi\right)=\rho I R \frac{\partial^{2} \psi}{\partial t^{2}}
$$

The added terms $f_{r}$ and $f_{\theta}$ are the external radial and tangential rib forces, respectively; there is no external moment. 


\section{A.5 Solution of the 3D Wave Equation (for Scalars)}

The three dimensional wave equation in cylindrical coordinates is given as

$$
\frac{\partial^{2} f}{\partial r^{2}}+\frac{1}{r} \frac{\partial f}{\partial r}+\frac{1}{r^{2}} \frac{\partial^{2} f}{\partial \theta^{2}}+\frac{\partial^{2} f}{\partial z^{2}}=\frac{1}{c^{2}} \frac{\partial^{2} f}{\partial t^{2}}
$$

with $c$ the material speed of sound. The solution to the wave equation for scalar harmonic functions can be found via the separation of variables method. Assuming the solution can be written as a combination of functions for each independent variable $(r, \theta, z, t)$, it takes the following form

$$
f(r, \theta, z, t)=R(r) \Phi(\theta) Z(z) T(t)
$$

Substituting the proposed solution into the wave equation results in

$$
-\frac{1}{c^{2}} T^{\prime \prime} R \Phi Z+R^{\prime \prime} \Phi Z T+\frac{1}{r} R^{\prime} \Phi Z T+\frac{1}{r^{2}} R \Phi^{\prime \prime} Z T+R \Phi Z^{\prime \prime} T=0
$$

where the dashes indicate a derivative with respect to the function's sole independent variable. Dividing by RФTZ leads to an equation with the temporal (T) functions isolated from the other variables. Therefore, the remaining equation is independent of $t$ and can be considered constant.

$$
\frac{1}{c^{2}} \frac{T^{\prime \prime}}{T}=\frac{R^{\prime \prime}}{R}+\frac{1}{r} \frac{R^{\prime}}{R}+\frac{1}{r^{2}} \frac{\Phi^{\prime \prime}}{\Phi}+\frac{Z^{\prime \prime}}{Z}=-k^{2}
$$

$k=\omega / c$ is the constant wavenumber. Ignoring the non-temporal part of the above, the equation for a single degree of freedom, $2^{\text {nd }}$ order differential equation remains. The solution is an exponential function, in which only the negative exponent has been kept.

$$
T^{\prime \prime}+\omega^{2} T=0 \rightarrow T(t)=C_{1} e^{-i \omega t}
$$

Returning to Equation [*], isolating the z-dependent terms means that the RHS is independent of $z$, and thus can be considered a constant.

$$
\frac{Z^{\prime \prime}}{Z}=-k^{2}-\frac{R^{\prime \prime}}{R}-\frac{1}{r} \frac{R^{\prime}}{R}-\frac{1}{r^{2}} \frac{\Phi^{\prime \prime}}{\Phi}=-k_{z}^{2}
$$

$\mathrm{k}_{\mathrm{z}}$ is the axial wavenumber. This forms another $2^{\text {nd }}$ order differential equation with an exponential solution, and the positive exponent is kept.

$$
Z^{\prime \prime}+k_{z}^{2} Z=0 \rightarrow Z(z)=C_{2} e^{i k_{z} z}
$$

Defining a new constant, $\mathrm{k}_{\mathrm{r}}^{2}=\mathrm{k}^{2}-\mathrm{k}_{\mathrm{z}}^{2}$ will help reduce some of the constants present. Isolating the $\theta$ terms of Equation [*] results in gives the following

$$
\frac{\Phi^{\prime \prime}}{\Phi}=-r^{2} k_{r}^{2}-r^{2} \frac{R^{\prime \prime}}{R}-r \frac{R^{\prime}}{R}=-n^{2}
$$


Again this leads to a $2^{\text {nd }}$ order differential equation with exponential solution.

$$
\Phi^{\prime \prime}+n^{2} \Phi=0 \rightarrow \Phi(\theta)=C_{3} e^{i n \theta}
$$

Unlike the temporal and axial solutions, the circumferential solution is periodic, $\Phi(\theta)=\Phi(\theta+2 \pi)$. Since $e^{0}=1=e^{2 \pi i n}, n$ must be an integer and the solution can be rewritten as a sinusoid; the $\sin$ term is disregarded.

$$
\Phi_{n}(\theta)=C_{3} \cos (n \theta), n=0,1,2,3 \ldots
$$

The circumferential solution Returning to Equation [*], the $r$-dependent terms remain, which can be rearranged into Bessel's Equation in $r$

$$
r^{2} R^{\prime \prime}+r R^{\prime}+\left(\left(k_{r} r\right)^{2}-n^{2}\right) R=0
$$

There are two linearly independent solutions to Bessel's Equation, made up of Bessel functions of the first and second kind.

$$
R_{n 1}(r)=C_{4} J_{n}\left(k_{r} r\right), \quad R_{n 2}(r)=C_{5} Y_{n}\left(k_{r} r\right)
$$

$J_{n}$ and $Y_{n}$ are a $n$th order Bessel functions of the first and second kind, respectively. $Y_{n}$ diverges as $r$ goes to 0 . For systems that require finite displacement at the origin (solid rods, fluids), this is not realistic and the $Y_{n}$ solution must be thrown out. Combining the four independent solutions, the original wave equation function, $f$, can be reconstructed. The solutions in $r$ and $\theta$, involving the integer $n$, must be summed. All the constants are combined into a single constant.

$$
f(r, \theta, z, t)=\sum_{n=0}^{\infty} A_{n} J_{n}\left(k_{r} r\right) \cos n \theta e^{i k_{z} z} e^{-i \omega t}
$$

Note that the Hankel function is a linear combination of $J_{n}$ and $Y_{n}$ and thus also solves the Bessel Equation. Therefore, the function $f$ can be expressed with a Hankel function of the first kind as well.

$$
\begin{gathered}
H_{n}^{(1)}(x)=J_{n}(x)+i Y_{n}(x), \quad H_{n}^{(2)}(x)=J_{n}(x)-i Y_{n}(x) \\
f(r, \theta, z, t)=\sum_{n=0}^{\infty} A_{n} H_{n}^{(1)}\left(k_{r} r\right) \cos n \theta e^{i k_{z} z} e^{-i \omega t}
\end{gathered}
$$

